University of Tennessee Health Science Center UTHSC Digital Commons

\title{
Insights into BTB-Cul3 Ubiquitin Ligases from the Structures of SPOP-Substrate Complexes
}

\author{
Min Zhuang \\ University of Tennessee Health Science Center
}

Follow this and additional works at: https://dc.uthsc.edu/dissertations

Part of the Amino Acids, Peptides, and Proteins Commons, Enzymes and Coenzymes Commons, Medical Biochemistry Commons, and the Medical Cell Biology Commons

\section{Recommended Citation}

Zhuang, Min , "Insights into BTB-Cul3 Ubiquitin Ligases from the Structures of SPOP-Substrate Complexes" (2009). Theses and Dissertations (ETD). Paper 328. http://dx.doi.org/10.21007/ etd.cghs.2009.0386.

This Dissertation is brought to you for free and open access by the College of Graduate Health Sciences at UTHSC Digital Commons. It has been accepted for inclusion in Theses and Dissertations (ETD) by an authorized administrator of UTHSC Digital Commons. For more information, please contact jwelch30@uthsc.edu. 


\title{
Insights into BTB-Cul3 Ubiquitin Ligases from the Structures of SPOP-Substrate Complexes
}

\author{
Abstract \\ Cullin-Ring ubiquitin ligases (CRLs) are E3 complexes that specifically recognize substrates through \\ substrate adaptors. In the largest CRL subfamily, Cul3 binds a BTB domain, and a protein-interaction \\ domain such as MATH recruits substrates for ubiquitination. Here we present biochemical and structural \\ analyses of the MATH and BTB domain containing protein, SPOP, which regulates diverse signaling \\ pathways. First, we identified a conserved SPOP Binding Consensus (SBC) motif in the transcriptional \\ regulator $\mathrm{Ci}$, the protein phosphatase Puc, and the chromatin component $\mathrm{MacroH} 2 \mathrm{~A}$. The SBC motif \\ specifically binds the MATH domain of SPOP, and is required for Puc ubiquitination in vitro and in vivo. \\ Structural determination and analysis of SPOPMATH in complex with peptides encompassing the SBC \\ motif revealed the molecular basis for recognition of diverse substrates by SPOP. Second, the dimeric \\ BTB domain of SPOP assembles into a dimer with Cul3, an interaction that is facilitated by a helical motif \\ that we term 3-box due to resemblance to F- and SOCS-boxes in other cullin-based E3s. 3-box is also \\ found in the BTB protein Gigaxonin and is predicted in a subset of BTB proteins. Furthermore, structures \\ of nearly full-length SPOP constructs indicate flexibility between the MATH and BTB domains, potentially \\ allowing regulation of diverse substrates. Those such as Puc with multiple SBCs may functionally interact \\ with a single SPOP dimer. All together, this study provides a molecular understanding of how MATH-BTB \\ proteins recruit substrates to $\mathrm{Cul} 3$, and how their dimerization and structural variability may facilitate \\ recognition and ubiquitination of diverse substrates. \\ Document Type \\ Dissertation \\ Degree Name \\ Doctor of Philosophy (PhD) \\ Program \\ Biomedical Sciences \\ Research Advisor \\ Brenda A. Schulman, Ph.D. \\ Keywords \\ BTB, Cul3, Ligase, MATH, SPOP, Ubiquitin

\section{Subject Categories} \\ Amino Acids, Peptides, and Proteins | Chemicals and Drugs | Enzymes and Coenzymes | Medical \\ Biochemistry | Medical Cell Biology | Medical Sciences | Medicine and Health Sciences
}




\title{
Insights into BTB-Cul3 Ubiquitin Ligases from the Structures of SPOP-Substrate Complexes
}

\author{
A Dissertation \\ Presented for \\ The Graduate Studies Council \\ The University of Tennessee \\ Health Science Center
}

In Partial Fulfillment

Of the Requirements for the Degree

Doctor of Philosophy

From The University of Tennessee

By

Min Zhuang

May 2009 
Copyright (C) 2009 by Min Zhuang

All rights reserved 


\section{DEDICATION}

To my parents, Jianhua Zhuang, Fengying Zhou and my husband, Haopeng Wang

for their love and support 


\section{ACKNOWLEDGEMENTS}

First, I would like to sincerely thank my mentor, Dr. Brenda A. Schulman, who opened the door to science for me, shared her experience, showed me an interesting world and, most importantly, helped me to better know myself. Brenda is an amazing scientist, from whom I learned a lot. I thank Brenda for teaching me to think critically and solve problems systematically, which will benefit me in the long term. Brenda is also a lovely person, as long as you do not break the $-80{ }^{\circ} \mathrm{C}$ fridge in the lab. We had a wonderful vacation together, which is one of my best personal experiences with her. Also, I would like to thank other committee members: Drs. Mary-Ann Bjornsti, Lawrence Pfeffer, Martine Roussel and Steve White for their assistance and time over the past four years. I'm so lucky to have had their advices at the early stage of my career.

My thanks are also given to Dr. Wade Harper (Harvard Medical School) for his insights into the project, Dr. Kevin White and Dr. Jiang Liu (the University of Chicago) for their contributions of in vivo experiments, Dr. Michal Hammel (Lawrence Berkeley National Laboratory) for SAXS analysis, Dr. Amanda Nourse and Brett Waddell (Hartwell Center) for protein interaction study and Dr. Jianmin Wang (Hartwell Center) for bioinformatics, without whose collaboration this work would not be complete. In addition, I want to thank Dr. Charles Ross and Dr. Darcie Miller of St. Jude Department of Structural Biology for remote data collection and software assistance.

Further, I would like to thank all the members of the Schulman lab, past and present, for their friendship and encouragement. I want to thank Danny Huang, who was practically my mentor for my first six months in the lab and has been a great friend and a role model of mine; Brett Waddell, who was so patient to train me when I barely understood English several years ago and who recently assisted me with tons of BIACORE experiments after he joined Hartwell Center; Helen Walden, who gave the lab the feel of family and accompanied me for my first synchrotron trip and led me to the world of crystallography; Dave Duda, who has been a source of technical expertise and has answered thousands of my "quick questions"; David Miller, who provides tremendous support to build the great environment for my studies; Jing Wang, who is always cheerful and a motivation for me to do physical exercise; Laura Borg, maybe Laura Scott now, whose birthday is the closest to mine and whose Thanksgiving cookies are always sweet; Danny Scott and Billy Dye, who helped me with my presentation skills and helped me better understand my project; Catherine Regni, who was a great desk neighbor and helped me with my proposal; Hari Kamadurai, who encouraged me to keep going; Judith Souphron and Asad Taherbhoy, fellow graduate students, with whom I shared a lot of things in common; Allison Williams and Woody Hunt, who brought so much fun to the lab; Steven Seyedin, who sent me directions in San Francisco during my interview; Allison Voss, who provided me a protein sample she made; and Shelia Bozeman, who has the warmest hug and fed me well with tons of donuts and candies. I want to thank Danny Scott and Dave Duda again for their proofreading of this dissertation. 
Finally, I would like to thank all my family members and friends who have given to me their love and support, especially my parents Jianhua Zhuang and Fengying Zhou, who have provided me invaluable educational opportunities; and my husband Haopeng Wang, who believed in me and supported me during the inevitable stressful and frustrating moments. 


\begin{abstract}
Cullin-Ring ubiquitin ligases (CRLs) are E3 complexes that specifically recognize substrates through substrate adaptors. In the largest CRL subfamily, Cul3 binds a BTB domain, and a protein-interaction domain such as MATH recruits substrates for ubiquitination. Here we present biochemical and structural analyses of the MATH and BTB domain containing protein, SPOP, which regulates diverse signaling pathways. First, we identified a conserved SPOP Binding Consensus (SBC) motif in the transcriptional regulator $\mathrm{Ci}$, the protein phosphatase Puc, and the chromatin component MacroH2A. The SBC motif specifically binds the MATH domain of SPOP, and is required for Puc ubiquitination in vitro and in vivo. Structural determination and analysis of SPOP ${ }^{\mathrm{MATH}}$ in complex with peptides encompassing the SBC motif revealed the molecular basis for recognition of diverse substrates by SPOP. Second, the dimeric BTB domain of SPOP assembles into a dimer with $\mathrm{Cul3}$, an interaction that is facilitated by a helical motif that we term 3-box due to resemblance to F- and SOCS-boxes in other cullin-based E3s. 3-box is also found in the BTB protein Gigaxonin and is predicted in a subset of BTB proteins. Furthermore, structures of nearly full-length SPOP constructs indicate flexibility between the MATH and BTB domains, potentially allowing regulation of diverse substrates. Those such as Puc with multiple SBCs may functionally interact with a single SPOP dimer. All together, this study provides a molecular understanding of how MATH-BTB proteins recruit substrates to $\mathrm{Cul3}$, and how their dimerization and structural variability may facilitate recognition and ubiquitination of diverse substrates.
\end{abstract}




\section{TABLE OF CONTENTS}

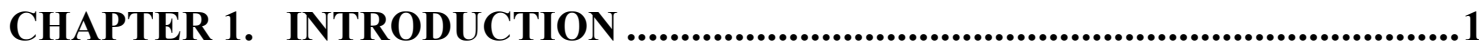

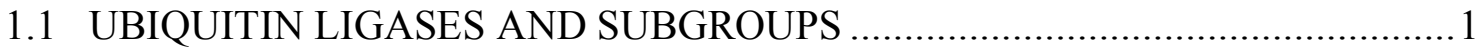

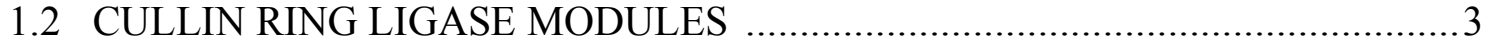

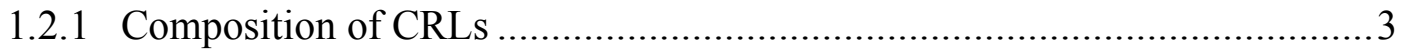

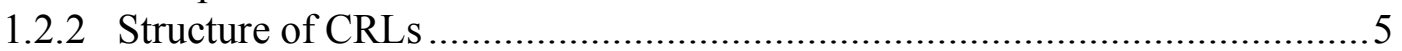

1.2.3 Regulation of CRLs by NEDD8 Modification............................................

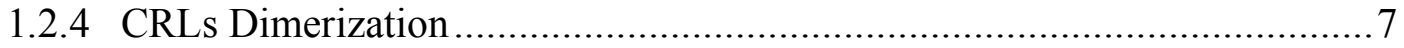

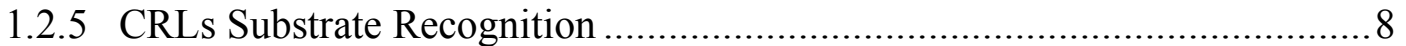

1.3 CUL3 SUBSTRATE ADAPTORS: BTB PROTEINS …....................................

1.3.1 BTB Domain: the Versatile Protein-Protein Interaction Domain ................. 9

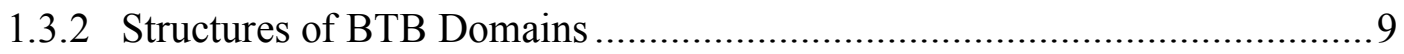

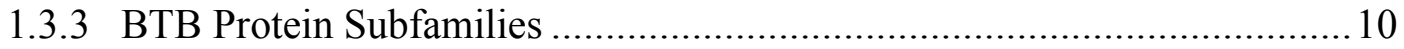

1.3.4 MATH-BTBs: the Largest BTB Subfamily ............................................ 10

1.4 SPOP: THE BEST-STUDIED MAMMALIAN MATH-BTB …….................... 10

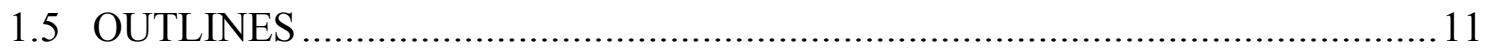

CHAPTER 2. EXPERIMENTAL PROCEDURES ...............................................13

2.1 IDENTIFICATION OF WORKABLE PROTEIN FRAGMENTS ……...............13

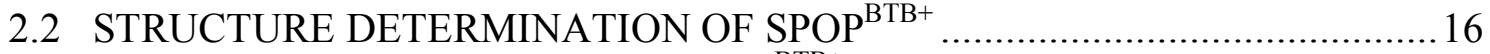

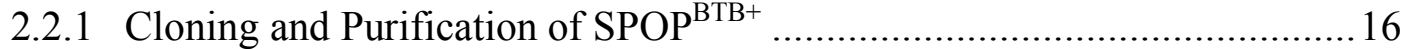

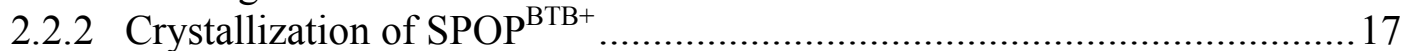

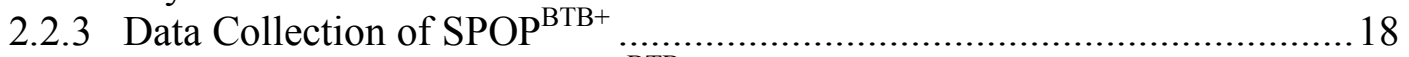

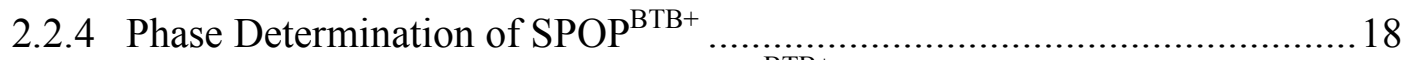

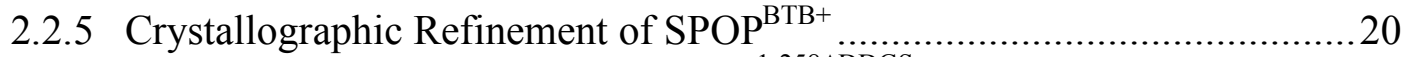

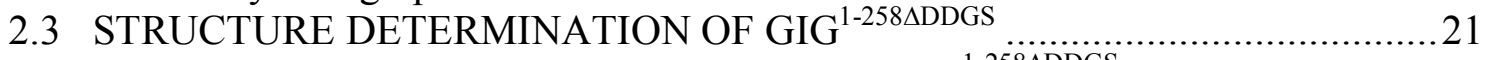

2.3.1 Cloning, Engineering and Purification of Gig ${ }^{1-258 \Delta D D G S} \ldots \ldots \ldots \ldots \ldots \ldots \ldots \ldots . . .21$

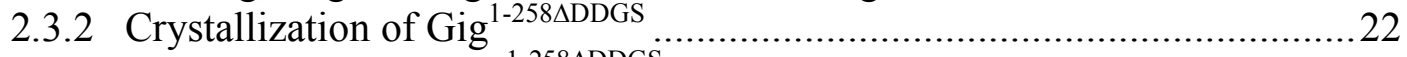

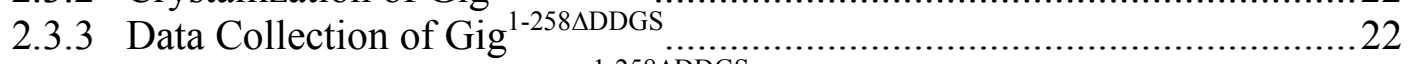

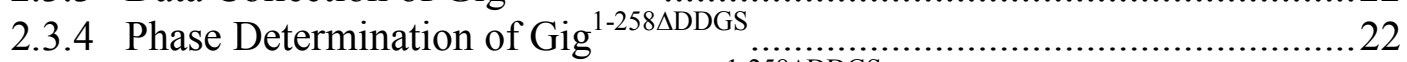

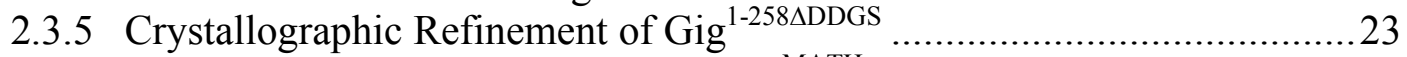

2.4 STRUCTURE DETERMINATION OF SPOP ${ }^{\mathrm{MATH}}{ }_{\text {-SBC COMPLEXES..............2 }}$

2.4.1 Cloning and Purification of $\mathrm{SPOP}^{\mathrm{MATH}}$..................................................2

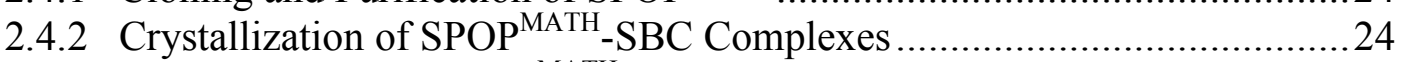

2.4.3 Data Collection of SPOP ${ }^{\mathrm{MATH}}{ }_{-\mathrm{SBC}}$ Complexes .....................................25

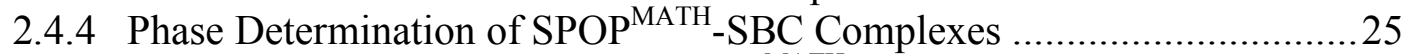

2.4.5 Crystallographic Refinement of SPOP ${ }^{\mathrm{MATH}}$-SBC Complexes ………….......2

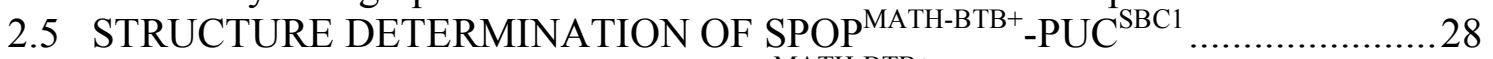

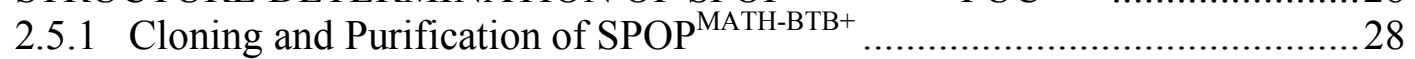

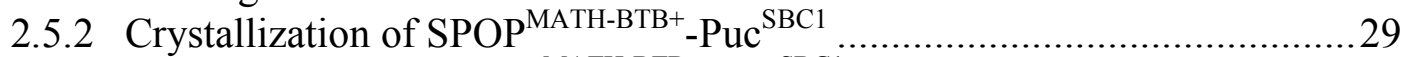

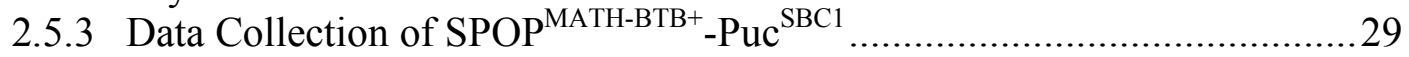




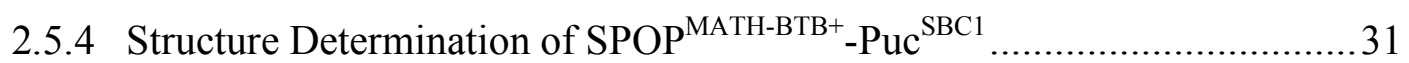

2.6 IDENTIFICATION OF THE SPOP BINDING CONSENSUS (SBC) ................31

2.6.1 Identification of a SPOP Binding Peptide in Puc .......................................31

2.6.2 Identification of a MacroH2A Sequence Required for Binding to SPOP....32

2.7 PROTEIN INTERACTION STUDIES ........................................................ 32

2.7.1 Interactions between SPOP ${ }^{\mathrm{MATH}}$ and SBC Sequences................................ 32

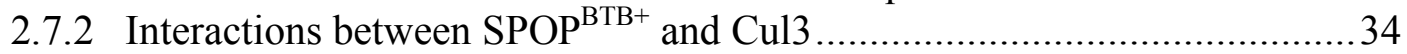

2.8 UBIQUITINATION ASSAY .............................................................. 36

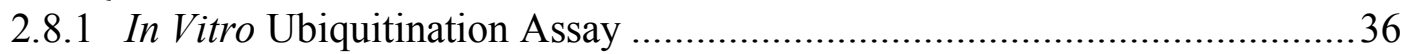

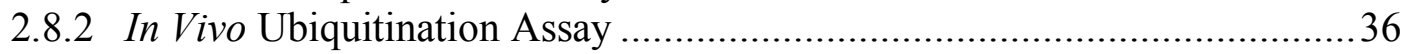

2.9 SMALL ANGLE X-RAY SCATTERING ANALYSIS ................................ 37

CHAPTER 3. SUBSTRATE RECOGNITION BY SPOP MATH DOMAIN.......38

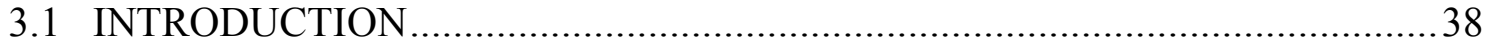

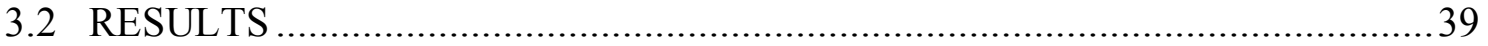

3.2.1 Identification of SPOP Binding Consensus (SBC) in Multiple

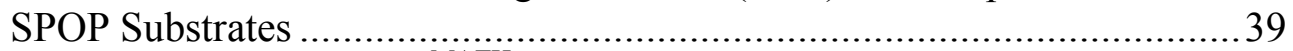

3.2.2 Structural Basis of SPOP ${ }^{\mathrm{MATH}}$-SBC Interactions .................................. 43

3.2.3 Mutagenesis Studies Validating SPOP ${ }^{\mathrm{MATH}}$-SBC Interactions ...................49

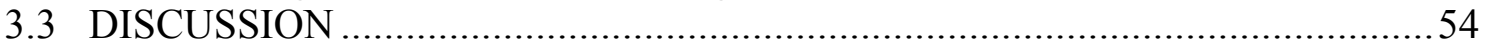

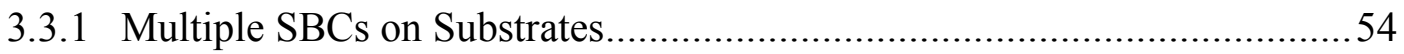

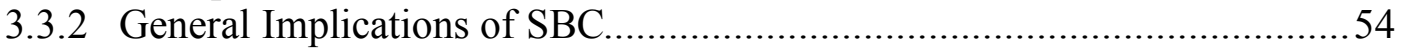

CHAPTER 4. STRUCTURE AND FUNCTION ANALYSIS OF SPOP ${ }^{\mathrm{BTB}+} \ldots . . . .58$

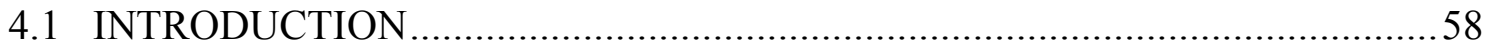

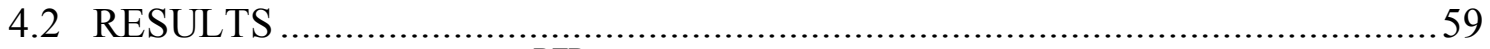

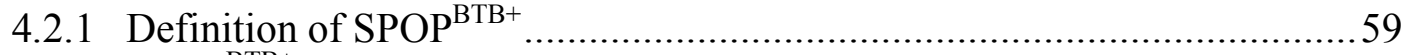

4.2.2 SPOP $^{\mathrm{BTB}+}$ Mediated Dimerization of $\mathrm{Cul3}$............................................59

4.2.3 The Skp1/ElonginC-like Core Domain of SPOP ${ }^{\mathrm{BTB}+}$...............................59

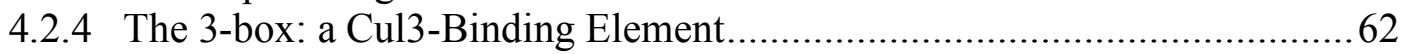

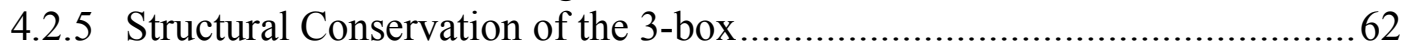

4.2.6 Predicted 3-box in a Subset of BTB Proteins ........................................62

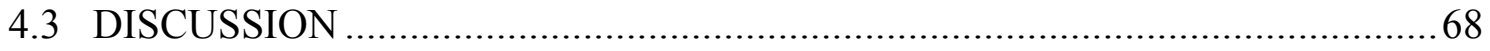

\section{CHAPTER 5. PUTTING THE MATH AND BTB DOMAINS TOGETHER: INSIGHTS INTO BTB-CUL3 UBIQUITIN LIGASES................71}

5.1 INTRODUCTION ............................................................................. 71

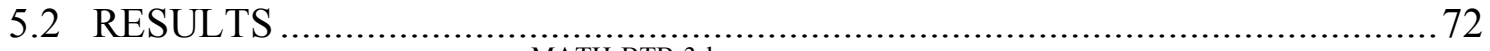

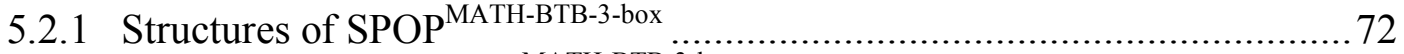

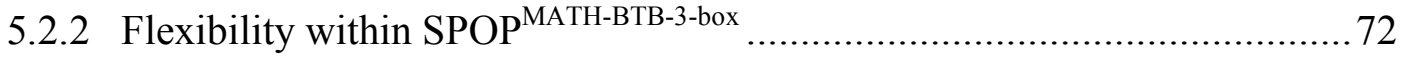


5.2.3 SPOP:Puc 2:1 Stoichiometry ....................................................................

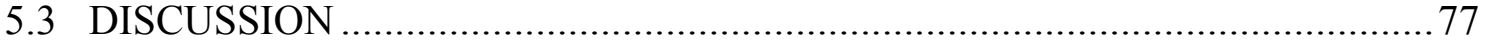

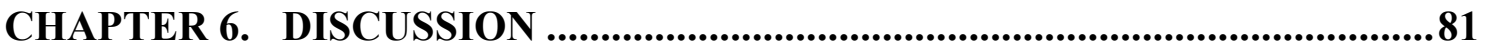

6.1 IMPLICATIONS FOR UBIQUITIN-MEDIATED REGULATION OF

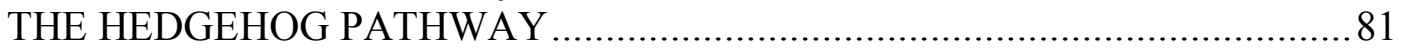

6.1.1 What Is the Role of the HIB-SBC Interaction in the Drosophila Hedgehog Pathway …….......................................................................... 81

6.1.2 What Is the Role of the SPOP-SBC Interaction in the Human

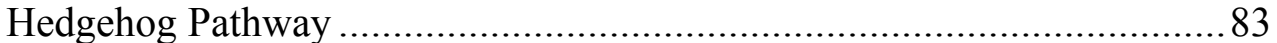

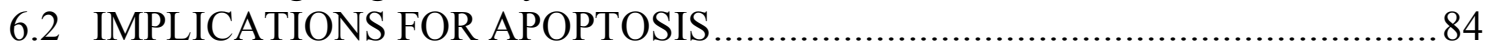

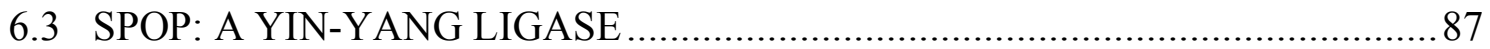

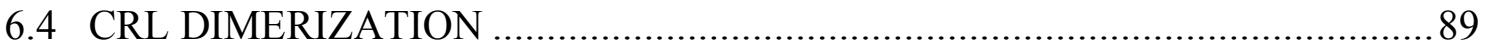

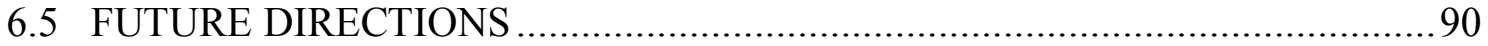

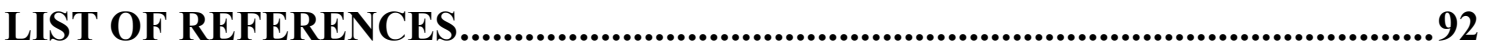

APPENDIX A. SUPPLEMENTARY DATA FOR STRUCTURAL STUDIES OF SPOP-CUL3 UBIQUITIN LIGASE ......................107

APPENDIX B. STUDIES OF INTERACTION BETWEEN E1 AND E2 OF THE NEDD8 PATHWAY.........................................................118

VITA 121 


\section{LIST OF TABLES}

Table 2.1. Protein fragments tested for crystallization ..................................... 14

Table 2.2. Structure data and refinement statistics of $\mathrm{SPOP}^{\mathrm{BTB}+}$ and

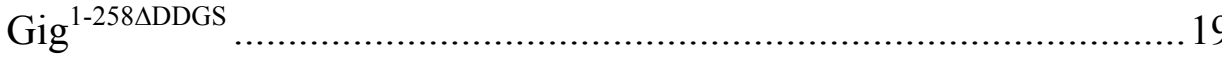

Table 2.3. Structure data and refinement statistics of SPOP ${ }^{\mathrm{MATH}}-\mathrm{SBC} \ldots \ldots \ldots \ldots \ldots . . . .26$

Table 2.4. Structure data and refinement statistics of $\mathrm{SPOP}^{\mathrm{MATH}-\mathrm{BTB}+}-\mathrm{Puc}^{\mathrm{SBC} 1} \ldots . .30$

Table 3.1. Hydrogen bonds between SPOP ${ }^{\mathrm{MATH}}$ and different SBC peptides.........52 


\section{LIST OF FIGURES}

Figure 1.1. Enzymatic cascade for ubiquitin modification ................................2

Figure 1.2. Cullin RING ligase modules...........................................................4

Figure 1.3. Structural model of a CRL ..................................................... 6

Figure 3.1. Identification of a SPOP-binding peptide in Puc............................. 40

Figure 3.2. Identification of a MacroH2A sequence required for binding to

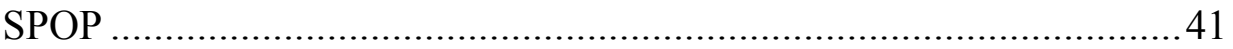

Figure 3.3. Identification of consensus sequences within four SPOP substrates ......42

Figure 3.4. Interactions between $\mathrm{SPOP}^{\mathrm{MATH}}$ and SBC peptides ...........................4 44

Figure 3.5. $\quad \mathrm{SBC}$ is required for Puc ubiquitination in vitro ................................45

Figure 3.6. Structure superimposition of $\mathrm{SPOP}^{\mathrm{MATH}}-\mathrm{Puc}^{\mathrm{SBC} 1}, \mathrm{TRAF} 2^{\mathrm{TRAF}}-\mathrm{CD} 40$

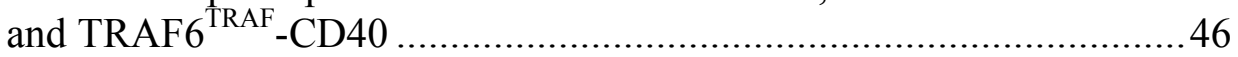

Figure 3.7. Crystal structures of $\mathrm{SPOP}^{\mathrm{MATH}}-\mathrm{Puc}^{\mathrm{SBC} 1}, \mathrm{SPOP}^{\mathrm{MATH}}-\mathrm{MacroH} 2 \mathrm{~A}^{\mathrm{SBC}}$

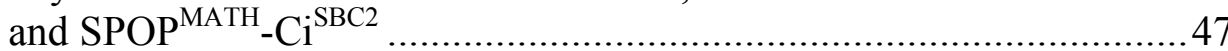

Figure 3.8. Structures of SPOP ${ }^{\mathrm{MATH}}$ complexed with two different peptides corresponding to the MacroH2A SBC

Figure 3.9. Structural comparison of three SBC peptides.....................................50

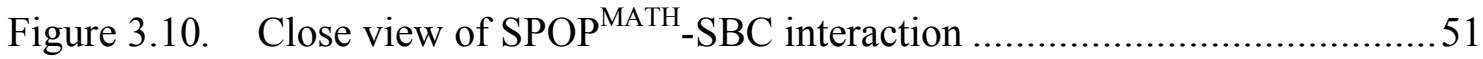

Figure 3.11. Validating SPOP ${ }_{-\mathrm{MATH}}{ }_{-\mathrm{SBC}}$ structures by binding studies....................53

Figure 3.12. Validating SPOP ${ }^{\mathrm{MATH}}-\mathrm{SBC}$ structures in cells .................................55

Figure 3.13. $\quad$ SPOP ${ }^{\mathrm{MATH}}$ does not bind phosphorylated SBC peptides ......................57

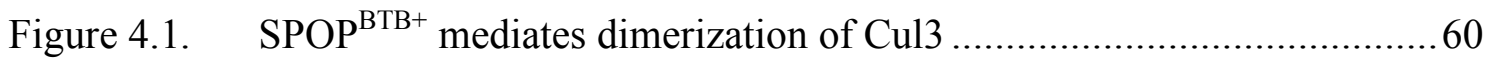

Figure 4.2. Structural superimposition of $\mathrm{SPOP}^{\mathrm{BTB}+}$, Skp1, EloC and other

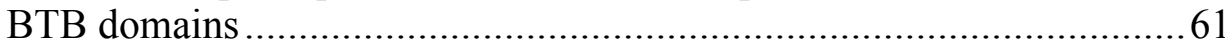

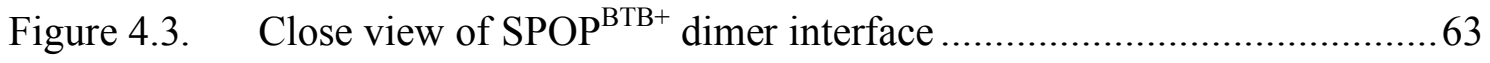


Figure 4.4. Identification of 3-box from $\mathrm{SPOP}^{\mathrm{BTB}+}$ crystal structure 64

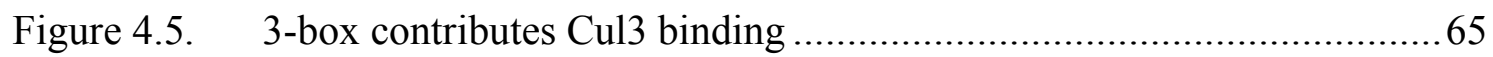

Figure 4.6. The 3-box structure is conserved in another BTB subfamily.................66

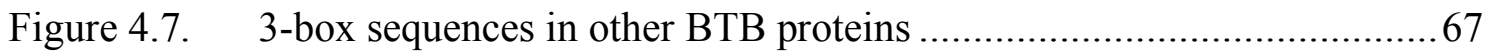

Figure 4.8. Identification of 3-box in a subset of BTB proteins ............................69

Figure 5.1. Overall architecture of $\mathrm{SPOP}^{\mathrm{MATH}-\mathrm{BTB}+}$ dimer....................................... 73

Figure 5.2. Superimposition over the BTB domains of $\mathrm{SPOP}^{\mathrm{MATH}-\mathrm{BTB}+}$ structures determined from crystals with slightly different unit cells......................74

Figure 5.3. $\mathrm{SPOP}^{\mathrm{MATH}-\mathrm{BTB}+}$ and $\mathrm{SPOP}^{\mathrm{N}-\mathrm{MATH}-\mathrm{BTB}+}$ display similar susceptibilities to proteolytic digestion by Endoproteinase Glu-C.................................. 75

Figure 5.4. SPOP MATH and BTB domains are flexibly tethered in solution..........76

Figure 5.5. A 1:2 substrate complex with the SPOP-Cul3 ubiquitin ligase...............78

Figure 5.6. Model of the SPOP-Cul3 ubiquitin ligase ...........................................79

Figure A.1. List of crystal structures determined in each CRL subfamily .............. 108

Figure A.2. Coomassie stain of representative purified proteins ............................ 109

Figure A.3. Puc ubiquitination in vitro depends on SPOP, Cul3, E1 and

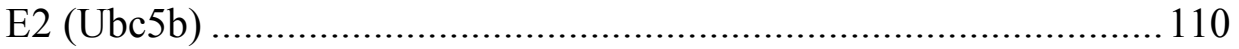

Figure A.4. SBC peptides bind SPOP MATH domain but do not bind HAUSP

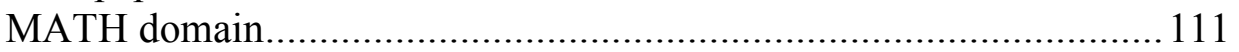

Figure A.5. Structure comparison of the D-domain and BTB domain.....................112

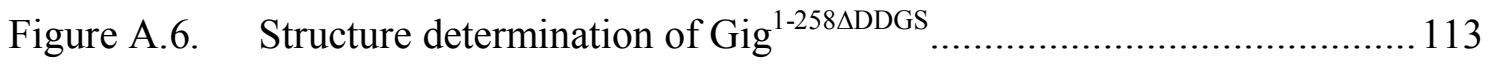

Figure A.7. Sequence alignment of SPOP from different species ..........................114

Figure A.8. Structural models of SPOP-Cul3 NEDD8-Rbx1-E2 complexes .........115

Figure A.9. Representative crystal pictures ……….......................................... 116

Figure A.10. Representative crystal diffraction images ......................................... 117 
Figure B.1. Alanine-scanning mutagenesis of Ubc12's core domain: effects of alanine substitutions on APPBP1-UBA3-catalyzed formation of the Ubc12 core NEDD8 thioester product complex 


\section{LIST OF ABBREVIATIONS}

$\begin{array}{ll}\text { BTB } & \text { bric-a-brack tramtrack broad } \\ \text { CRL } & \text { cullin RING ligase } \\ \text { Cys } & \text { cysteine } \\ \text { EOM } & \text { ensemble optimization method } \\ \text { HECT } & \text { homologous to E6-AP carboxyl terminus } \\ \text { MATH } & \text { meprin associated TRAF homology } \\ \text { POZ } & \text { poxvirus zinc finger } \\ \text { RING } & \text { really interesting new gene } \\ \text { SAD } & \text { single wavelength anomalous dispersion } \\ \text { SAXS } & \text { small angle X-ray scattering analysis } \\ \text { SCF } & \text { Skp1-Cull-F-box } \\ \text { Ser } & \text { tyrosine } \\ \text { Thr } & \text { serine } \\ \text { TRAF } & \text { threonine } \\ \text { Tyr } & \text { ubiquitin }\end{array}$




\section{CHAPTER 1. INTRODUCTION}

\subsection{UBIQUITIN LIGASES AND SUBGROUPS}

Ubiquitin is a small protein (76 amino acids) that can be covalently attached to substrate proteins [1-3]. Ubiquitin modification, also referred to as ubiquitination, regulates a variety of cellular activities, such as transcriptional regulation [4-6], cell signaling [7-11], DNA repair [12, 13] and apoptosis [14-16]. Ubiquitin can modify substrate proteins in different ways to elicit different functions. For example, the attachment of a single ubiquitin to a substrate lysine is known as mono-ubiquitination. Mono-ubiquitination is usually involved in protein targeting and endocytosis $[17,18]$. Ubiquitin can also be conjugated to one of the seven Lys residues on itself, thus forming different poly-ubiquitin chains. The most well characterized poly-ubiquitin chains are K48-linked and K63-linked, the former mediating proteasome-dependent degradation [19-21] and the later mediating cellular signaling [7, 22, 23].

Ubiquitin modifies a substrate via E1-E2-E3 enzyme cascades. First, ubiquitin is activated by the ubiquitin activating enzyme E1 and then conjugated to the catalytic Cys on ubiquitin conjugating enzyme E2 [24-26]. In the final step of this enzyme cascade, ubiquitin ligase, also referred to as E3, specifically recognizes the substrate protein and catalyzes ubiquitin conjugation to the substrate (Fig 1.1) [27, 28].

In the human genome, there are two E1s, tens of E2s and hundreds of E3s recognizing thousands of substrates. In general, there are two types of E3s. HECT (Homologous to E6-AP Carboxyl Terminus) E3s and RING (Really Interesting New Gene) E3s. HECT E3s catalyze ubiquitin transfer by forming a covalent E3-ubiquitin intermediate prior to transferring ubiquitin to substrate proteins [29, 30]. Many characterized HECT E3s are involved in cancer development, such as Smurf mediated degradation of Smad proteins $[31,32]$ and E6-AP mediated degradation of p53 (reviewed in $[33,34]$ ). Human genome encodes 28 HECT domain-containing proteins. By contrast, RING E3s catalyze ubiquitin transfer directly from E2 to substrate without a covalent intermediate $[35,36]$. In humans, there are more than 400 RING E3s. RING E3s can be further divided into three families with increased protein size: simple RINGs, Cullin RING Ligases (CRLs) and Anaphase Promoting Complex (APC). Simple RINGs are single polypeptides that contain the E2 recruiting RING domain. A well-studied simple RING E3 is c-Cb1, which mediates ubiquitination and subsequent degradation of PDGF, EGF receptor tyrosine kinases, thus attenuating signaling [35]. The structure of $\mathrm{c}-\mathrm{Cbl}$ in complex with the $\mathrm{E} 2 \mathrm{UbcH} 7$ has been determined, which provides the molecular basis for RING-E2 interaction and serves as the template for various RING-E2 interaction modeling [37]. CRLs are multi-component protein complexes. In humans, there are more than 300 CRLs. CRLs not only comprise the largest number of RING E3s, but also represent the largest group among all E3s [38-41]. APC is the largest RING E3, playing important roles in cell cycle regulation. In addition to the CRL-like functional core, APC contains at least 11 subunits, which are poorly characterized at the molecular level (reviewed in [42, 43]). 


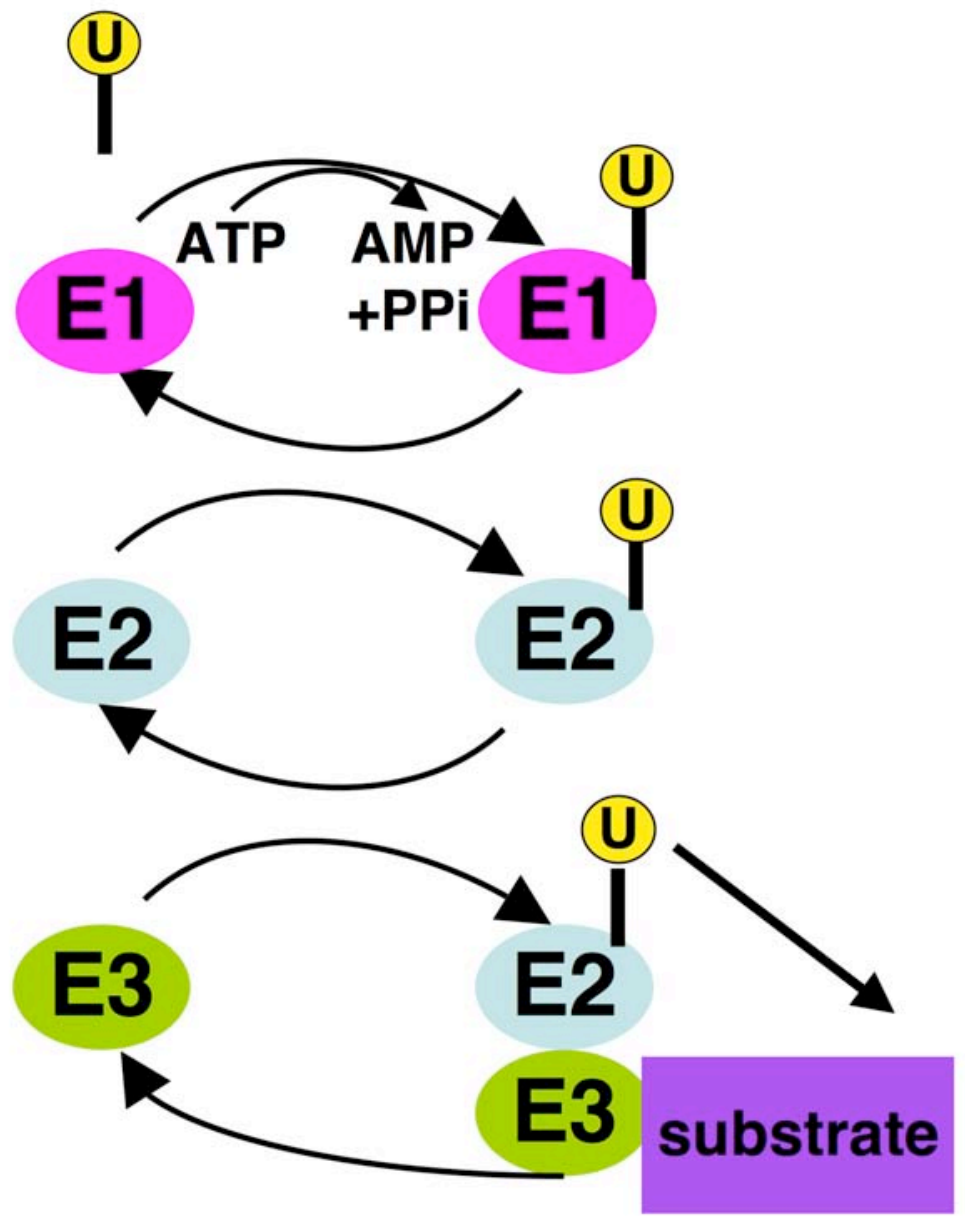

Figure 1.1. Enzymatic cascade for ubiquitin modification. Ubiquitin is shown as yellow circle with a black stick representing the $\mathrm{C}$ terminal tail of ubiquitin. E1 (ubiquitin activating enzyme) is in light magenta, E2 (ubiquitin conjugating enzyme) is in cyan, E3 (ubiquitin ligase) is in green and substrate is in purple. 


\subsection{CULLIN RING LIGASE MODULES}

\subsubsection{Composition of CRLs}

CRLs are multi-subunit protein complexes. The crystal structures of a number of CRL components determined in last several years revealed that CRLs are modular complexes [38, 44-48]. Minimally, a CRL contains a substrate adaptor, cullin, and RING box protein (Rbx). Cullin is the scaffold subunit with an extended conformation. The $\mathrm{N}$ terminus of cullin binds the substrate adaptor, which in turns, interacts with substrates. The $\mathrm{C}$ terminus of cullin binds Rbx, which recruits Ub-loaded E2s (Fig 1.2). CRLs catalyze ubiquitination by bringing Ub-loaded E2 and substrate into close proximity.

In the human genome, there are five major CRL subfamilies built on different cullins with different sets of exchangeable substrate adaptors (Fig 1.2). The best understood CRL is the Cul1-based ubiquitin ligase, also referred as SCF (Skp1-Cul1-F-box) ubiquitin ligase. Skp1 serves as an adaptor that simultaneously binds the F-box motif of the F-box protein and sequences near the $\mathrm{N}$ terminus of Cul1. In turn, F-box proteins contain additional protein interaction domains that recruit the substrate into the Cul1-Skp1-F-box protein complex, thereby facilitating ubiquitination of the target via the catalytic core assembled on the $\mathrm{C}$ terminus of $\mathrm{Cul1}[45,46]$. Cul2 and Cul5 share a similar organization, wherein the Skp1-like protein Elongin $\mathrm{C}$ recruits members of the SOCS-box family of proteins to Cul2/5 $[49,50]$. The F- or SOCS-box links the substrate-binding domain to Skp1 or Elongin C. In contrast, Cul3 employs BTB proteins as substrate specific adaptors. BTB proteins are characterized by containing the BTB domain, a protein interaction and dimerization motif first identified in Drosophila transcriptional repressors Bricabrack-Tramtrack-Broad Complex [51]. The BTB domain was later demonstrated to have structural homology with the cullin-binding domain of Skp1, and to bind Cul3 through motifs analogous to that seen with the Cul1-Skp1 complex [38, 52-57]. Many BTB domain-containing proteins also contain additional protein interaction domains, some of which have been implicated in recognition of ubiquitination targets [10,58-61]. Thus, BTB proteins appear to merge the functional properties of Skpland F-box proteins or Elongin C and SOCS-box into a single polypeptide chain, without an intervening F- or SOCS-box.

Some CRLs have additional subunits. For example, CKS1 binds adaptor Skp2 and promotes recruitment of the substrate P2 $7^{\text {Kip1 }}$ to the Skp1-Cul1-Skp2 ligase [62, 63]. Elongin B is always found associated with Elongin C in Cul2/5-based CRLs. DDA1, which binds Cul4 adaptor DDB2, is identified as a core subunit of multiple Cul4-based CRLs [41]. 

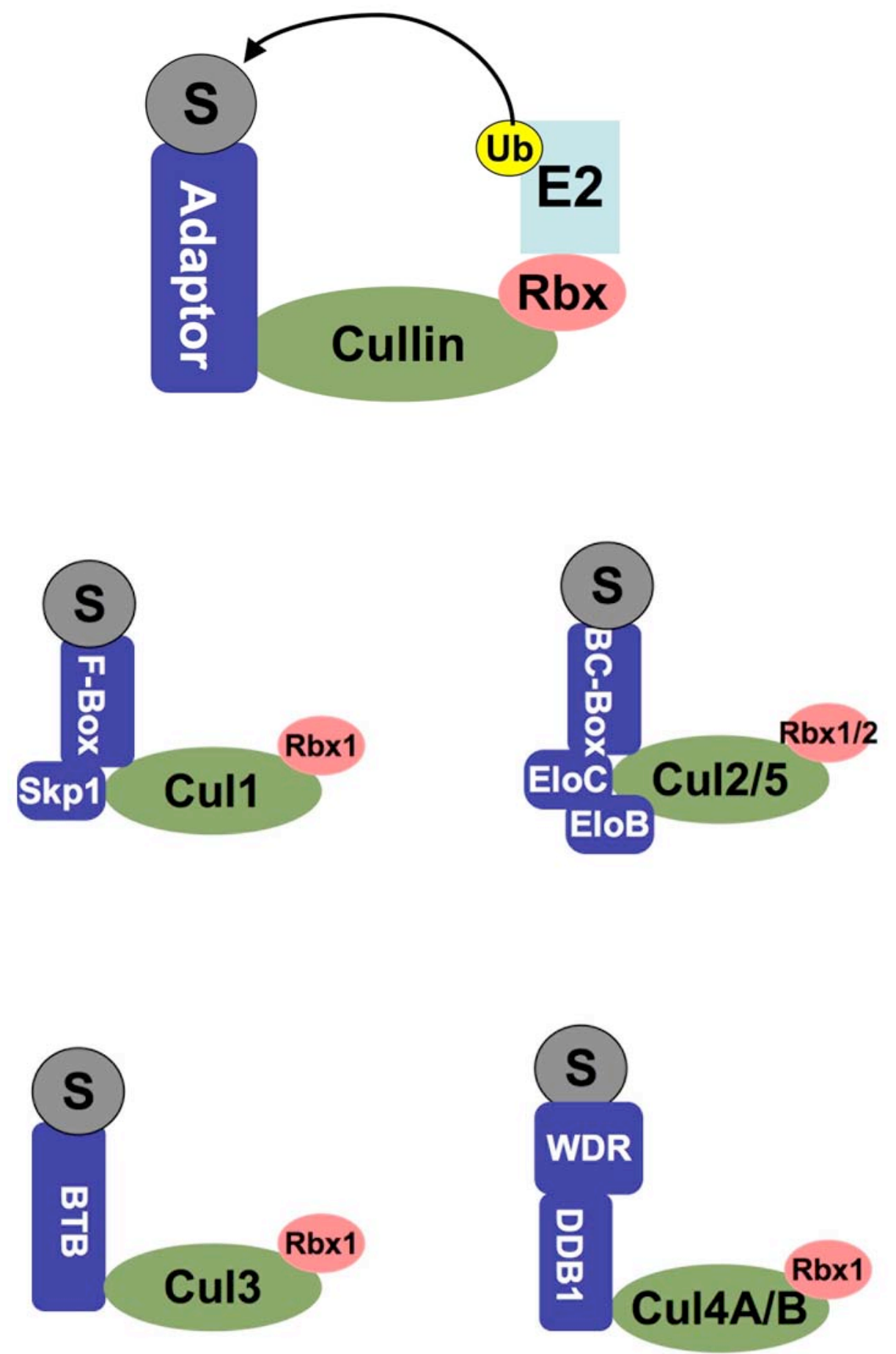

Figure 1.2. Cullin RING ligase modules. A schematic view of CRL modular is on the top. Components of four major families of CRLs are shown individually (substrate adaptors in blue, cullins in limegreen, Rbxs in salmon, substrates in grey, ubiquitin conjugating ezyme E2 in cyan with its bound ubiquitin in yellow). 


\subsubsection{Structure of CRLs}

Two CRL complexes, Skp1-Cul1-F-box ${ }^{\text {Skp2 }}$ [45] and DDB1-Cul4-SV5-V [13] have been crystallized to reveal the architecture of CRLs. In these complexes, Cull and $\mathrm{Cul} 4$ adopt an elongated structure with helical repeats at the $\mathrm{N}$ terminus and a globular domain at the $\mathrm{C}$ terminus. The substrate adaptors (Skp1-F-box ${ }^{\text {Skp2 }}$ and DDB1) bind to the first $\mathrm{N}$-terminal helical repeat of $\mathrm{Cull}$ or Cul4, helices 2 and 5 in particular. These helices are conserved in $\mathrm{Cul} 2 / 5$ and $\mathrm{Cul} 3$. Although there are no crystal structures of $\mathrm{Cul} 2 / 5$ or Cul3 in complex with their corresponding adaptors, mutational analyses demonstrate that $\mathrm{Cul} 2 / 5$ and $\mathrm{Cul} 3$ interact with Elongin $\mathrm{C}$ and BTB protein in a similar manner. For example, mutations in helices 2 and 5 of $\mathrm{Cul} 3$ disrupt binding to the BTB protein Mel-26 [53]. Similarly, deletion of helix 2 of Cul5 abolishes its interaction with Elongin C [64].

In addition to cullin-containing CRL complex structures, several structures of CRL adaptors in complexes with substrates have been determined, including Cul1 adaptors Skp1-Cdc4 bound to cyclin E [48], Skp1- $\beta \operatorname{TrCP}$ bound to $\beta$-catenin [44], Skp1-Fbw7 bound to cyclin E [65], Cul2 adaptors Elongin B-Elongin C-VHL bound to Hif-1 $\alpha$ [66] and Elongin B-Elongin C-SOCS3 bound to gp130 [67] (see Appendix A, Fig A.1 for a complete list). A common feature of these structures is they all contain the cullin-binding subunit Skp1 or Elongin C. Structurally, Skp1 and Elongin C share a similar core fold. Thus, models of CRLs bound to substrate can be generated by superimposing Skp1 or Elongin C onto the Skp1-Cul1-F-box complex (Fig 1.3).

The globular domain at the $\mathrm{C}$ terminus of $\mathrm{Cul1}$ and $\mathrm{Cul} 4$ binds the RING protein $\mathrm{Rbx} 1$ through the intermolecular $\beta$ sheet. The RING domain from c-Cbl in complex with the E2 UbcH7 can be docked on Rbx1 to complete the structure model [37] (Fig 1.3). In this model, as well as other CRL models with different substrate adaptors, there is a gap of about $50 \AA$ between E2 and substrate. However, to form an iso-peptide bond between the lysine of the substrate and ubiquitin, the lysine needs to reach the catalytic cysteine on E2. Thus, this distance must change during ubiquitination.

What causes the change of this distance? There are several hypotheses. First, additional CRL subunits, such as CKS1 and DDA1, may bridge the gap between E2 and substrates. However, the crystal structure of Skp1-Skp2-CKS1-p27 fails to fill the gap and furthermore, many substrates are ubiquitinated without a requirement for additional CRL subunits. It has also been suggested that the distance between the adaptor binding site and the target lysine on substrate may fill the gap. Ubiquitination by $\beta$-TrCP is most efficient when a lysine is present 9 to 13 residues upstream from the binding site [44]. However, fully extending 13 residues are still not sufficient to reach $\sim 50 \AA$.

Alternatively, it is tempting to speculate that a part of the CRL structure is flexible, which allows the distance to change. It is unclear which part of CRL could be less rigid, since mutations designed to introduce flexibility into Cull disrupt its function in vitro [45]. Rigid structural coupling is also important for substrate adaptors [44, 46, 48]. Take $\beta-\mathrm{TrCP}$ for example: the linker domain between F-box and substrate binding WD40 domain consists of four $\alpha$ helices that interact with both the F-box and the broad face of 

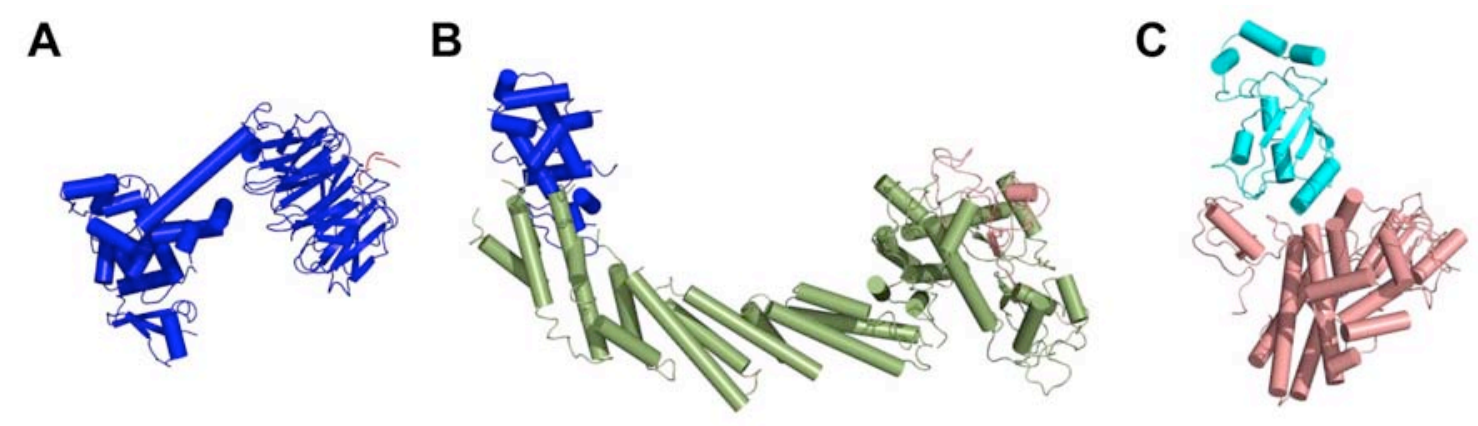

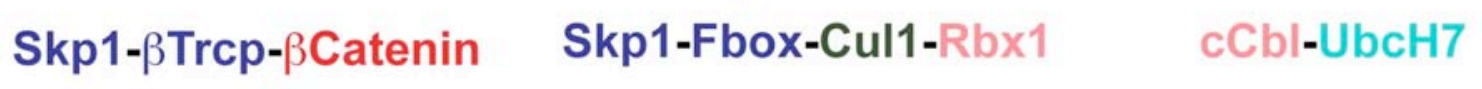

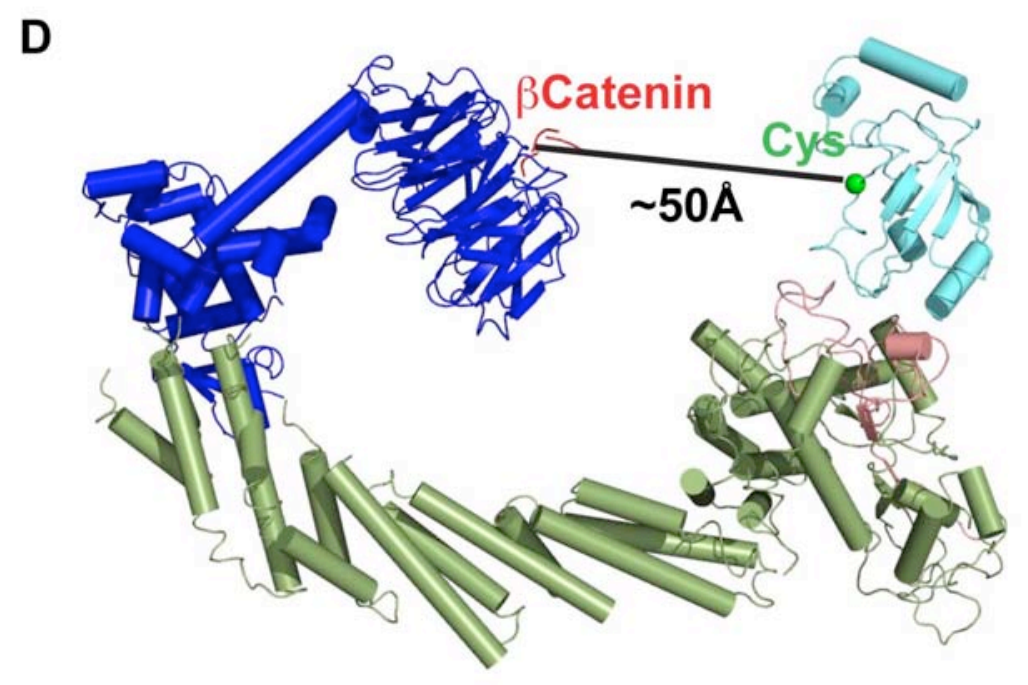

Figure 1.3. Structural model of a CRL. A structure model of CRL (D) is made from three crystal structures: (A) Skp1- $\beta$ Trcp- $\beta$-catenin (PDB ID: $1 \mathrm{P} 22)$, (B) Skp1-Cul1-Fbox ${ }^{\text {SKP2 }}$ (PDB ID: 1LDK) and (C) c-Cbl-UbcH7 (PDB ID: 1FBV). Skp1 and $\beta$-Trcp are in blue, Cul1 is in limegreen, $\mathrm{Rbx} 1$ and c-Cbl are in pink and $\mathrm{UbcH7}$ is in cyan. The catalytic Cys on $\mathrm{UbcH} 7$ is shown as a green sphere and the $\beta$-catenin peptide is shown as red cartoon. 
the WD40 propeller. Mutations in this linker region designed to affect rigid coupling disrupted protein function in vivo [48]. DDB1 is an exception, because it adopts different conformations in different crystal structures $[12,13,68]$. So it seems that substrate adaptors can have certain flexibility. The major contribution to gap closing is from the Rbx1RING domain. Recent structural studies from our lab [69], together with other biochemical and molecular studies [70,71], revealed flexibility within Rbx1 upon neddylation and provided molecular foundations for closing the gap between ubiquitin-loaded E2 and the substrate.

\subsubsection{Regulation of CRLs by NEDD8 Modification}

NEDD8 is an ubiquitin-like protein that can be covalently attached to substrate proteins $[72,73]$. Neddylation is critical for cell cycle progression and essential for viability in many species, including $M$. musculus [74], C. elegans $[75,76], D$. melanogaster [77] and $A$. thaliana [78]. The primary targets of NEDD8 modification are cullins, the scaffold proteins of CRLs. NEDD8 modifies a conserved lysine on the cullin $\mathrm{C}$ terminal globular domain and stimulates CRL-catalyzed ubiquitin transfer from E2 to targets both in vivo and in vitro [79-82]. It also serves to block association with CAND1, an inhibitor of CRLs [83-85].

The crystal structure of the neddylated $\mathrm{C}$ terminal part of $\mathrm{Cul} 5$ and small angle $\mathrm{X}$-ray scattering analysis of the neddylated $\mathrm{C}$ terminus of Cul1 reveal a dramatic conformational change of cullin induced by neddylation [69]. This conformational change results in the freeing of the Rbx1 RING domain from interactions with cullin. Therefore, in a neddylated CRL, Rbx1 is flexibly tethered to the $\mathrm{C}$ terminus of cullin and adopts multiple conformations. E2 bound to freed Rbx1 can be positioned to a close proximity to substrate lysine. Rbx1 orientational flexibility may also have implications in ubiquitination variability. It allows substrates of varying sizes and with varying positions of target lysines to be accommodated by the same cullin.

\subsubsection{CRLs Dimerization}

A large number of CRLs dimerize through substrate adaptors. Studies of Fbw7 and its budding yeast homologues Cdc4 reveal that dimerization of those proteins is essential in vivo [86]. In addition, dimerization of the BTB protein Keap1 is critical for Nrf2 degradation [87]. In vitro, dimerization of $\mathrm{Fbw} 7$ is found to be important for both initiation and elongation of ubiquitin chains [65].

Substrate adaptors dimerize through domains different from their substrate binding domain. For example, Fbw $7 / \mathrm{Cdc} 4$ and $\beta$-TrCP homo- or hetero-dimerize through the D-domain, a 45 amino acid motif immediately $\mathrm{N}$ terminal to the F-box [65, 86, 88]. By contrast, BTB proteins, such as Mel-26, RhoBTB2 and Keap1, dimerize through the BTB domain, the Skp1/ElonginC-like Cul3 binding domain [53, 55, 57, 87, 89-92]. Unlike the $\mathrm{N}$ terminal D-domain in F-box proteins, the BTB domain can be at 
the $\mathrm{N}$ terminus or $\mathrm{C}$ terminus, or centrally located in the BTB proteins. D-domains are highly conserved in WD40 containing F-box proteins and BTB domains are present in all BTB proteins, so it's expected that many Cul1- and Cul3-based CRLs are dimeric. Since there is no evidence for the dimerization of SOCS-box proteins, Cul2- and Cul5-based CRL may not dimerize.

Studies of Fbw7/Cdc4-mediated Cull dimerization suggest that dimerization provides more conformational variability to CRLs. First, dimerized CRLs may bring two ubiquitin-loaded E2s in close proximity to the substrate, and thus provide geometry for trans-ubiquitination in addition to cis-ubiquitination. Second, the varying distances between each ubiquitin-loaded E2 and substrate may provide geometry for ubiquitin chain formation. It is also suggested that CRL dimerization may play other roles, such as facilitating substrate recognition and optimally orientation of the target lysines.

However, no solid evidence has surfaced to support these claims. Further studies need to focus on various CRL dimers, especially those uncharacterized BTB-Cul3 dimers, to examine whether a common mechanism is applicable to both CRL subfamilies.

\subsubsection{CRLs Substrate Recognition}

CRL substrates typically contain degradation signals that can be recognized by substrate adaptors. A degradation signal or "degron" is defined as "minimal element within a protein that is sufficient for recognition and degradation by a proteolytic apparatus" [93]. To date, two types of degrons, phosphodegrons and oxygen-dependent degrons, have been identified among CRL substrates. Ubiquitination of most substrates by Cul1-based ubiquitin ligases requires phosphorylation on specific Ser or Thr residues $[63,94-96]$. Tyr phosphorylation is required for substrate recognition by some Cul2 adaptors, such as SOCS2 and SOCS3 [67, 97-99]. Another Cul2 adaptor, VHL, specifically associates with hydroxylated prolines $[5,47,66,100]$. Crystallographic analyses of adaptor-substrate complexes demonstrate that substrate adaptors make direct contact with the phosphorylated or hydroxylated amino acid. A short peptide containing the degron is sufficient for binding.

A single substrate may possess multiple degrons. Sic1, a substrate of Cdc4, contains nine phosphodegrons $[48,101]$. The Fbw7 substrate cyclin E contains minimally two phosphodegrons $[65,88,102]$. Experiments suggest that multiple degrons are required for those substrates being degraded in vivo. However, it still remains unclear why multiple sites are required since one degron is sufficient for binding.

Specific substrate adaptors can bind multiple substrates in various signaling pathways. For example, $\beta$-TrCP mediates the ubiquitination of I $\mathrm{B}$, an inhibitor of $\mathrm{NF}-\kappa \mathrm{B}$ pathway, relieving the transcription factor NF- $\kappa \mathrm{B}$ from its inhibition $[80,103$, 104]. It can also target $\beta$-catenin for ubiquitination, inhibiting $\beta$-catenin binding to Tcf/Lef transcription factors and transcriptional activation of a number of oncogenes [11, $105,106]$. Moreover, $\beta$-TrCP is linked to mitosis by mediating ubiquitination of Emi1 [96]. All $\beta$-TrCP substrates contain a conserved phosphodegron sequence "DSGXXS", 
where serines are phophorylated and " $\mathrm{X}$ " can be any amino acid. $\beta$ - $\mathrm{TrCP}$ binds the same motif on different substrates.

Various structures of substrate adaptors have been determined in complex with substrate peptide. In those crystal structures, substrate peptides are always in an extended form. The interactions between adaptor and the substrate are mainly mediated by extensive hydrogen bonds and electrostatic interactions [44, 48, 62, 65]. Hydrophobic interactions are also observed in some substrate binding but do not dominate in substrate recognition [44].

\subsection{CUL3 SUBSTRATE ADAPTORS: BTB PROTEINS}

The human genome encodes more than 180 proteins that contain recognizable BTB domains [107]. These BTB proteins can potentially serve as interchangeable substrate adaptors for Cul3-based CRLs, making BTB-Cul3 the largest CRL subfamily.

\subsubsection{BTB Domain: the Versatile Protein-Protein Interaction Domain}

The BTB domain (also called the POZ domain) is a protein interaction and dimerization domain first identified in Drosophila transcriptional repressors "Bric-a-brack, Tramtrack and Broad Complex" (BTB) [51] and many poxvirus zinc finger (POZ) proteins [108]. Subsequently, BTB domains were found throughout eukaryotes with quite versatile roles in a broad range of cellular functions, including transcriptional regulation $[109,110]$, ion channel assembly [111] and substrate specific ubiquitination $[4,60,61]$. The best studied BTB domain is from an anti-apoptotic transcriptional factor $\mathrm{Bcl} 6$, which plays critical roles in many immunological processes. Bcl6 activity requires the recruitment of histone deacetylases through co-repressors, including BCOR (Bc16 interacting co-repressor), NcoR and SMRT. These co-repressors bind in a lateral groove at the interface of the two chains of the Bcl6 dimer $[110,112]$. This binding site is far from the predicted Cul3 binding site on Bcl6.

\subsubsection{Structures of BTB Domain}

Crystal structures of several BTB domains, including Bcl6 ${ }^{\mathrm{BTB}}, \mathrm{PLZF}^{\mathrm{BTB}}, \mathrm{LRF}^{\mathrm{BTB}}$ and $\mathrm{BACH}^{\mathrm{BTB}}$, have been determined by X-ray crystallography $[109,110,112,113]$. The overall structures of BTB domains are similar in spite of poor sequence conservation. The BTB domain contains a short three-strand $\beta$ sheet and a five-helix cluster, which demonstrate structural homology with Skp1 and Elongin C. In addition to the Skp1/ElonginC-like core, the BTB domain contains additional N-terminal elements that mediate homo-dimerization of BTB with an extensive hydrophobic interface. 


\subsubsection{BTB Protein Subfamilies}

BTB domains are found in association with several other classes of protein interaction domains, including Zinc-finger (ZF), MATH, Kelch, ankyrin and other poorly characterized domains. BTB-ZF proteins are best characterized as transcription factors with both structural and biochemical studies [109, 110, 112-115]. To date, only BTB proteins containing MATH and Kelch domains have been linked to substrate targeting by Cul3 $[4,55,60,61,116]$ and it remains unclear precisely how many BTB domains engage Cul3 in vivo. The best understood Cul3 adaptor is the BTB-Kelch protein Keap1, a central regulator of the oxidative stress pathway in multi-cellular eukaryotes.

Keap1-Cul3 is responsible for rapid turnover of the Nrf2 transcription factor in the cytoplasm, thereby impeding the activation of oxidative stress-responsive genes $[6,61]$. Structural studies of the Keap1 Kelch domain and Nrf2 peptide complex have identified a basic surface on the propeller structure generated by Kelch repeats that interacts with an acidic motif in Nrf2 [117]. Little is known as to how other Cul3-associated BTB proteins recognize their substrates.

\subsubsection{MATH-BTBs: the Largest BTB Subfamily}

The MATH-BTB module, in which a MATH (meprin-associated TRAF homology) domain is at the $\mathrm{N}$ terminus of the BTB domain, is the largest BTB family, and is also one of the most abundant among all proteins. Indeed, the MATH-BTB module is the $10^{\text {th }}$ most abundant two-domain combination encoded by 113 archael, bacterial, and eukaryotic genomes [118]. Both plants and C. elegans have greatly expanded the repertoire of MATH-BTB proteins through diversification of the MATH domain, and it has been suggested that this module is used for antiviral protective mechanisms in these organisms [57, 119].

Despite the broad importance of MATH-BTBs, little is known about how any MATH-BTB protein recognizes a substrate, or bridges a substrate and Cul3 for ubiquitination. The first evidence that MATH-BTB proteins function in protein degradation came from the identification of C. elegans Mel-26 as a component of a Cul3 dependent E3 responsible for the turnover of the Mei-1 meiosis-specific microtubule severing protein during the first mitotic division $[53,55,57]$.

\subsection{SPOP: THE BEST-STUDIED MAMMALIAN MATH-BTB}

In mammals, the closest Mel-26 ortholog is SPOP (speckle-type POZ protein), which exhibits a discrete speckled pattern in cells [120]. The mRNA encoding human SPOP is found expressed in eight tissues examined, including heart, brain, lung, liver, kidney, pancreas, placenta and skeletal muscle [120]. Recently, SPOP expression was examined in various cancers. The mRNA levels of SPOP are altered in different medulloblastoma subgroups [121]. Tissue microarray screening for SPOP expression in 
18 cancer types from different organs reveal high expression of SPOP in $99 \%$ of clear cell renal carcinomas, the most prevalent form of kidney cancers [58].

The full length SPOP, consisting of 374 amino acids, has two domains: the MATH domain (residues 28-166) and the BTB domain (residues 172-297). The MATH domain is a subtype of the TRAF (tumor necrosis factor-receptor associated factor) domain. It mediates interactions between SPOP and substrate proteins, such as Puckered and $\mathrm{MacroH} 2 \mathrm{~A}$. A dimeric SPOP BTB domain is required for $\mathrm{Cul3}$ binding (more information of the SPOP MATH domain in Chapter 3 and the SPOP BTB domain in Chapter 4).

SPOP has been linked to ubiquitination of divergent substrates in diverse signaling pathways. SPOP/Cul3 mediates ubiquitination of MacroH2A, thereby controlling deposition of MacroH2A on the inactive X-chromosome [122, 123], and ubiquitination of Daxx, thereby controlling Daxx-dependent transcriptional repression of pro-apoptotic proteins such as p53 [116, 124]. More recently, a Drosophila ortholog of Mel-26, called HIB or Roadkill, has been linked to multiple signaling systems. During development, HIB/Roadkill negatively regulates the hedgehog pathway by promoting the degradation of the $\mathrm{Ci}$ transcription factor. This process is independent of the $\mathrm{SCF}^{\text {slimb }}$ dependent processing of $\mathrm{Ci}$ to its repressive form $[4,125,126]$. In addition, HIB/Roadkill negatively regulates Tumor Necrosis Factor signaling in the Drosophila eye by promoting turnover of the MAPK phosphatase Puckered (Puc) [58]. SPOP and HIB/Roadkill share conserved protein sequences, especially at the MATH domains, which are $94 \%$ identical between Homo Sapiens and Drosophila. Consistent with this, over expression of human SPOP in Drosophila can rescue the development defect caused by deletion of HIB/Roadkill [125].

Despite significant progress in understanding the biological pathways in which the SPOP family of proteins function, little is known as to how target specificity is achieved. Known targets of the SPOP family lack obvious domains that would serve to link them to a common degradation mechanism, and unlike most SCF substrates, posttranslational modifications are not known to control turnover of SPOP substrates. Moreover, SPOP substrates are targeted for degradation in multiple locations within the cell, suggesting that localization is not a major source of specificity.

\subsection{OUTLINES}

To gain insight into BTB-Cul3 ubiquitin ligases, we tried to determine the crystal structure of a BTB protein. Because the crystallization of a protein is unpredictable, we started the project with multiple BTB proteins, including SPOP, Gig, Keap1 and RhoBTB2. In this work, we reports the results obtained from the study of SPOP. SPOP has two functional domains. We dissected the function of each domain and then put them together to better understand the SPOP-Cul3 ubiquitin ligase. 
In Chapter 2, we describe the experimental procedures for a crystallographic, biophysical, and biochemical characterization of SPOP. In Chapters 3-5, we report results and discuss briefly SPOP substrate binding, SPOP Cul3 binding, and SPOP overall architecture. In Chapter 6, we discuss the biological implications and future directions of our studies. 


\section{CHAPTER 2. EXPERIMENTAL PROCEDURES}

\subsection{IDENTIFICATION OF WORKABLE PROTEIN FRAGMENTS}

To obtain milligram quantities of purified proteins for crystallization purposes, bacterial and baculovirus-insect cell based expression systems are predominant choices for generating recombinant proteins. The former generally has advantages in product yield and generation time. The limitation is that the environment within bacteria may not be suitable for expressing some eukaryotic proteins, in part due to the lack of eukaryotic chaperones that assist protein folding and the lack of post-translational modifications. The baculoviral system may provide a more "friendly" environment to eukaryotic proteins, but the yields are often low compared to bacterial expression systems. Furthermore, it takes nearly a month to obtain baculoviruses, as compared to a few days to generate a sequence-verified bacterial expression construct. To obtain high yield of recombinant human proteins, we tried both systems in this study.

Various approaches, including fusing different tags at either the $\mathrm{N}$ or $\mathrm{C}$ terminus, and using different expression cell lines, have been tried to obtain full length SPOP, Gig, Puc, Cul3 and other proteins involved in this study. However, we did not obtain sufficient quantities of these proteins for crystallization screening. We did not even obtain enough for limited proteolysis, which is one of the most common methods to identify intact protein domains. Therefore, we designed potentially crystallizable constructs based on secondary structure prediction methods. A standard approach to engineer truncated protein fragments for crystallization is to remove flexible unstructured regions, without disrupting intact protein domains. We have used several structure prediction programs, including PONDR (Predictor of Naturally Disordered Regions) to predict unstructured regions and PHD (www.predictprotein.org) to predict the secondary structures. We designed the constructs based on these predictions. The full list of constructs tried is summarized in Table 2.1. In addition, we tried different protein tags, including Glutathione S-transferase (GST), maltose-binding protein (MBP), and hexa-histidine (His), because each tag has advantages and disadvantages. Although GST often facilitates solubility, GST also dimerizes in solution, and this might block the proper folding of fused proteins; although MBP also often facilitates solubility, it is a 44 $\mathrm{kDa}$ protein, which contributes the majority of the product if a smaller protein is fused; although the His-tag is small, elution of His-tagged proteins requires imidazole, which we find often causes some ion-bound proteins to precipitate. Thus, we made various constructs for the same protein because of the unpredictable crystallization nature of each protein. The constructs tested are summarized in Table 2.1. In summary, we have made 50 protein constructs from 12 BTB proteins, tested the expression of each construct and co-expression of BTB protein and Cul3, identified four soluble protein fragments, crystallized three of these and determined eight distinct crystal structures (four containing the same protein fragment bound to different synthetic peptides). In this chapter, only the protein fragments that crystallized are discussed further. 
Table 2.1 Protein fragments tested for crystallization.

\begin{tabular}{|c|c|c|c|c|c|c|}
\hline Protein & Residues & Tag & Expression & Folding & Crystal & Diffraction \\
\hline \multirow[t]{4}{*}{ Keap1 } & $67-180$ & GST & - & & & \\
\hline & $67-260$ & GST & - & & & \\
\hline & $67-289$ & GST & - & & & \\
\hline & $67-312$ & GST & - & & & \\
\hline \multirow[t]{5}{*}{ Gigaxonin } & $20-130$ & GST & - & & & \\
\hline & $20-208$ & GST & +++ & $\mathrm{F}$ & & \\
\hline & $20-238$ & GST & + & $\mathrm{F}$ & & \\
\hline & $20-258$ & GST & +++ & $\mathrm{F}$ & & \\
\hline & $1-258$ & GST & +++ & $\mathrm{F}$ & yes & + \\
\hline \multirow[t]{5}{*}{ VACWR180 } & BTB only & GST & ++ & & & \\
\hline & $\mathrm{BTB}+1 \mathrm{H}$ & GST & + & & & \\
\hline & $\mathrm{BTB}+4 \mathrm{H}$ & GST & - & & & \\
\hline & $\mathrm{BTB}+6 \mathrm{H}$ & GST & - & & & \\
\hline & $\mathrm{BTB}+7 \mathrm{H}$ & GST & - & & & \\
\hline \multirow[t]{16}{*}{ SPOP } & $1-166$ & GST & +++ & $\mathrm{F}$ & & \\
\hline & $23-166$ & GST & +++ & $\mathrm{F}$ & & \\
\hline & $28-166$ & GST & +++ & $\mathrm{F}$ & yes & +++ \\
\hline & $172-297$ & GST & ++ & $\mathrm{F}$ & & \\
\hline & $172-337$ & GST & +++ & $\mathrm{F}$ & yes & +++ \\
\hline & $28-337$ & GST & ++ & $\mathrm{F}$ & & \\
\hline & $23-337$ & GST & ++ & $\mathrm{F}$ & & \\
\hline & $\mathrm{N}-\mathrm{C}$ & GST & - & & & \\
\hline & $28-166$ & His-MBP & +++ & $\mathrm{F}$ & & \\
\hline & $28-337$ & His-MBP & +++ & $\mathrm{F}$ & yes & +++ \\
\hline & $23-337$ & His-MBP & +++ & $\mathrm{F}$ & & \\
\hline & N-337 & His-MBP & +++ & $\mathrm{F}$ & & \\
\hline & $\mathrm{N}-350$ & His-MBP & +++ & - & & \\
\hline & $28-360$ & His-MBP & +++ & - & & \\
\hline & N-360 & His-MBP & +++ & - & & \\
\hline & $\mathrm{N}-\mathrm{C}$ & His-MBP & - & & & \\
\hline \multirow[t]{6}{*}{ RhoBTB2 } & $\mathrm{N}-\mathrm{C}$ & GST & - & & & \\
\hline & $252-C$ & GST & - & & & \\
\hline & $252-488$ & GST & +++ & - & & \\
\hline & $252-600$ & GST & +++ & - & & \\
\hline & $252-665$ & GST & +++ & - & & \\
\hline & $489-600$ & GST & - & & & \\
\hline
\end{tabular}


Table 2.1. Protein fragments tested for crystallization (Continued).

\begin{tabular}{lllcccc}
\hline Protein & Residues & Tag & Expression & Folding & Crystal & Diffraction \\
\hline RhoBTB2 & $489-665$ & GST & - & & & \\
& $489-C$ & GST & - & & & \\
KBTB1 & N-255 & GST & +++ & - & & \\
KBTB2 & N-223 & GST & - & & & \\
KBTB3 & N-243 & GST & - & & & \\
KBTB4 & N-243 & GST & - & & & \\
KBTB5 & N-260 & GST & - & & & \\
KBTB6 & N-260 & GST & - & & & \\
KBTB7 & N-261 & GST & - & & \\
& & & & & \\
Cu13 & $1-198$ & His (N) & - & & \\
& $1-266$ & His (N) & - & & \\
& $1-198$ & His (C) & - & & \\
& $1-266$ & His (C) & - & & \\
& $1-133$ & GST & - & & & \\
& $1-206$ & GST & - & & & \\
& $1-384$ & GST & +++ & F & \\
& $16-384$ & GST & +++ & F & \\
\hline
\end{tabular}




\subsection{STRUCTURE DETERMINATION OF SPOP ${ }^{\mathrm{BTB}+}$}

\subsubsection{Cloning and Purification of SPOP ${ }^{\mathrm{BTB}+}$}

The attempt to express full-length human SPOP in bacterial expression systems failed, and to obtain sufficient protein for crystallization purposes, several SPOP fragments were tested for their expression and solubility. We were able to purify SPOP $^{172-337}$, which contains the BTB domain sequence and extra C-terminal residues that are predicted to be folded. SPOP ${ }^{172-337}$ was cloned into the bacterial expression vector pGEX4T-1, where the Schistosoma japonicum Glutathione S-transferase (GST) is fused upstream of the cloning site. The vector containing the coding region for SPOP ${ }^{172-337}$ was transformed into E.coli BL21 (DE3) GOLD. Expression of SPOP ${ }^{172-337}$ was initiated through the inoculation of $200 \mathrm{ml}$ of L-Broth (LB) media containing a final concentration of $200 \mu \mathrm{g} / \mathrm{ml}$ ampicillin. The starter media was incubated at $37{ }^{\circ} \mathrm{C}$ overnight in a rotary shaker at 200 revolutions per minute (rpm). $10 \mathrm{ml}$ of the cultured media was then used to inoculate each of the twelve 1-liter aliquots of $\mathrm{LB}$ media containing a final concentration of ampicillin of $200 \mu \mathrm{g} / \mathrm{ml}$. The 1-liter cultures were allowed to grow at $37^{\circ} \mathrm{C}$ at 200 rpm for five to six hours yielding an $\mathrm{OD}_{600}$ of 0.8 to 0.9 . Induction of T7 RNA polymerase was done through the addition of isopropyl $\beta$-D-thiogalactopyranoside (IPTG) to a final concentration of $0.6 \mathrm{mM}$. For SeMet labeled protein expression, M9 minimal media with $0.5 \%$ glycerol was used instead of LB media. $50 \sim 70 \mathrm{mg}$ of each individual amino acids (Thr, Ser, Val, Leu, Ile, Phe) were added to cultures to shut down endogenous bacterial Met production $30 \mathrm{~min}$ prior to SeMet protein induction by addition of IPTG and $50 \mathrm{mg}$ of SeMet to each of the 1-liter cultures. The cultures were then incubated at $16^{\circ} \mathrm{C}$ in a rotary shaker overnight ( 15 to 18 hours) to allow protein expression. Cells in each of the 1-liter culture were harvested and re-suspended in $5 \mathrm{ml}$ Tris buffer containing $50 \mathrm{mM}$ Tris (pH 7.6), $200 \mathrm{mM} \mathrm{NaCl}, 5 \mathrm{mM}$ Dithiothreitol (DTT), and $2.5 \mathrm{mM}$ phenylmethylsulphonyl fluoride (PMSF), and then lysed by sonication on ice.

The general protein purification strategy for SPOP ${ }^{172-337}$ includes four steps: (1) affinity chromatography using Glutathione Sepharose 4B to pull down GST tagged protein; (2) cleavage between the GST protein and SPOP ${ }^{172-337}$ by the protease thrombin; (3) ion exchange chromatography to separate GST and purified proteins based on their different isoelectric point; (4) size exclusive chromatography to further purify proteins by eliminating different-sized contaminants. The isoelectric point is 4.5 for SPOP ${ }^{172-337}$ and 6.7 for GST. At neutral $\mathrm{pH}$, both proteins are negatively charged, so an anion exchanger was used for ion exchange chromatography. Cell lysates from 12 liters of cultures were loaded manually on a gravity column packed with $20 \mathrm{ml}$ Glutathione Sepharose 4B resin with a flow-through rate of approximately $1 \mathrm{ml}$ per minute. The resins were then washed with $100 \mathrm{ml}$ lysis buffer and the GST-SPOP ${ }^{172-337}$ was eluted with $50 \mathrm{mM}$ Tris (pH8.0), $200 \mathrm{mM} \mathrm{NaCl}, 10 \mathrm{mM}$ reduced Glutathione and 5mM DTT. Thrombin was added to GST-SPOP ${ }^{172-337}$ to a mass ratio of 1:300 and calcium chloride to a final concentration of $2.5 \mathrm{mM}$. Thrombin cleavage proceeded at $4{ }^{\circ} \mathrm{C}$ overnight. GST and SPOP ${ }^{172-337}$ mixtures, which contain $200 \mathrm{mM} \mathrm{NaCl}$ in buffer, were diluted with $50 \mathrm{mM}$ Bis-Tris 
Propane (BTP) buffer containing no salt, to make the final concentration of $\mathrm{NaCl}$ at 50 $\mathrm{mM}$. Diluted proteins were loaded on a $30 \mathrm{ml}$ SOURCE Q column, using an AKTA fast protein liquid chromatography (FPLC) system. GST flowed through and SPOP ${ }^{172-337}$ was eluted with buffer containing $50 \mathrm{mM}$ BTP, $5 \mathrm{mM}$ DTT and 150 to $220 \mathrm{mM} \mathrm{NaCl}$ during gradient elution. The protein was concentrated and further purified with SD200 gel filtration chromatography, using an AKTA FPLC. Finally, SPOP ${ }^{172-337}$ was split into 25-35 microliter aliquots, flash frozen in liquid nitrogen, and stored in buffer containing $20 \mathrm{mM}$ Tris (pH 7.6), $150 \mathrm{mM} \mathrm{NaCl}$ and $5 \mathrm{mM}$ DTT at $-80^{\circ} \mathrm{C}$. The SeMet labeled SPOP $^{172-337}$ was purified using the same methods. $10 \mathrm{mM}$ DTT was added to all buffers used for SeMet proteins purification, in contrast to the $5 \mathrm{mM}$ DTT used for native protein purifications.

\subsubsection{Crystallization of SPOP ${ }^{\mathrm{BTB}+}$}

Initial crystallization screening was done using the hanging drop method over 360 commercially available and homemade conditions with wild type SPOP ${ }^{\mathrm{BTB}+}$ protein. Crystal drops were prepared by mixing $1 \mu \mathrm{l}$ of $20 \mathrm{mg} / \mathrm{ml}$ protein with $1 \mu \mathrm{l}$ of precipitant solution. The drops were equilibrated by vapor diffusion against $500 \mu \mathrm{l}$ of precipitant solution at $4{ }^{\circ} \mathrm{C}$. Initial crystals grew in several drops with two types of shapes: the needle-like crystals grew in 20-25\% PEG3350, 0.1 M Bis-Tris pH 5.5, and the clusters of hexagonal crystals grew in 1.5-2.5 M ammonium sulfate, 0.1 M Bis-Tris pH 6.0. However, those crystals were not suitable for data collection because the needle-like crystals were extremely thin (less than $30 \mu \mathrm{m}$ ) and the hexagonal crystals were not single. To obtain single and larger crystals, we optimized the crystals by screening around the initial conditions with different precipitant concentrations, different $\mathrm{pH}$ buffers, different commercial and homemade additives, and different temperatures. The optimized hexagonal crystals grew in $1.7 \mathrm{M}\left(\mathrm{NH}_{4}\right)_{2} \mathrm{SO}_{4}, 0.1 \mathrm{M}$ Bis-Tris $\mathrm{pH} 6.0,6 \%$ sucrose, $5 \mathrm{mM}$ DTT at room temperature in three days and were harvested with the same precipitant solution with additional $20 \%$ glycerol as cryoprotectant. The optimized needle-like crystals grew larger than initial ones in 21-22 \% PEG 6000, 0.1 M Bis-Tris, $0.05 \mathrm{M}$ $\mathrm{NaCl}, 10 \mathrm{mM}$ DTT, pH 5.5, but were still not suitable for crystallographic structure determination. These crystals were further used as sources of seeds for seeding experiments. The placement of crystal seeds into a condition that contains slightly lower precipitant concentration may help crystals grow from the seeds and prevent unwanted random nucleation. A combination of two types of seeding, streak seeding and macro seeding was used to optimize the needle-like crystals with the SeMet $\mathrm{SPOP}^{\mathrm{BTB}+}$. First, streak seeding was used. New drops containing $10 \%$ less precipitant and SeMet $\mathrm{SPOP}^{\mathrm{BTB}+}$ were set up and incubated for 3 hours at $4{ }^{\circ} \mathrm{C}$. A whisker was used to pick up small crystal fragments after smashing the crystals from old drops and was streaked through the fresh mixture. New crystals grew along the streaking line after 3 days. Next, macro seeding was used. The crystals obtained by streak seeding were diluted with $10 \mu 1$ well buffer and $0.2 \mu \mathrm{l}$ was pipetted into another freshly incubated $2 \mu \mathrm{l}$ drop. Higher quality SeMet SPOP ${ }^{\mathrm{BTB}+}$ crystals grew in one week with better three-dimension shape and larger size than native ones. These crystals were harvested with $20 \%$ glycerol and flash frozen in liquid nitrogen. 


\subsubsection{Data Collection of SPOP ${ }^{\mathrm{BTB}+}$}

X-ray diffraction datasets were collected at Brookhaven National Laboratory, National Synchrotron Light Source, Beamline X25. A fluorescence scan was performed prior to data collection to determine the peak energy/wavelength for Se. The data were collected at $\lambda=0.9792 \AA$ with an exposure time of 5 seconds per frame, and 1.0 degree oscillation angles. The frames were recorded on an ADSC Q315 CCD detector. The unit cell parameters were determined using the HKL2000 software [127]. The SeMet $\mathrm{SPOP}^{\mathrm{BTB}+}$ crystals displayed better diffraction than native ones and were used for all aspects of structure determination. The crystals belonged to the triclinic space group $\mathrm{P} 1$ with unit cell dimension of $a=36.8 \AA, b=88.7 \AA, c=88.7 \AA, \alpha=90.8^{\circ}, \beta=89.3^{\circ}, \gamma=89.9^{\circ}$. 360 frames were collected. A total of 37,547 reflections were integrated and scaled with HKL2000 to a resolution of $2.5 \AA$. Data processing parameters are summarized in Table 2.2 .

\subsubsection{Phase Determination of SPOP ${ }^{\mathrm{BTB}+}$}

Single-wavelength anomalous dispersion (SAD) was used to determine the phase of $\mathrm{SPOP}^{\mathrm{BTB}+}$ diffraction. SAD, similarly to multiple-wavelength anomalous dispersion (MAD) method, has been developed to determine the phases based on the anomalous signals of heavy atoms, which is produced when part of the energy is absorbed by heavy atoms [128]. Anomalous scattering of heavy atoms produces absorption vector $\mathrm{f}^{\prime \prime}$ and dispersion vector $\mathrm{f}^{\prime}$. MAD produces de novo phase information but requires longer exposure time of crystals to X-ray for a full dataset collected at three wavelengths, which usually damages crystals. SAD, which utilizes a single wavelength, requires less data collection but has the inherent "Hand" problem: it cannot distinguish differences between mirror images. In addition, $\mathrm{SAD}$ is reported to have advantages when anomalous signals are low.

To determine the structure of SPOP ${ }^{\mathrm{BTB}+}$, the automated crystallographic structure solution program SOLVE was used [129]. SOLVE determines the positions of the anomalous scatters, calculates and refines the phases, and finally generates electron density maps. The inputs for SOLVE included the space group, unit cell parameters, the scale file from HKL2000 [127], wavelength of $0.9792 \AA, f^{\prime}$ of $-7.59, f^{\prime \prime}$ of 4.22 ( $f^{\prime}$ and $\mathrm{f}^{\prime \prime}$ were calculated from the fluorescence scan of the SeMet labeled SPOP ${ }^{\mathrm{BTB}+}$ crystal), and the number of expected Se atoms. Since each SPOP molecule contains eight SeMets, and an estimated four molecules in the asymmetric unit (which equals unit cell in this case), we set up the SOLVE program to search for $50 \mathrm{Se}$ atoms, which includes an additional 18 atoms in case the program picks up wrong sites. 23 out of 32 expected Se sites were found by SOLVE with occupancy scores better than 0.1 . The poor electron density map generated based on this solution indicated that this might be a mirror solution, as the "hand" is established randomly by SOLVE. To obtain the correct phases, the reverse Se sites generated by the first run of SOLVE were input for the second run. Continuous experimental maps with clear solvent boundaries were generated by the second run of SOLVE. The phases were further improved with the software RESOLVE, 
Table 2.2. Structure data and refinement statistics of $\mathrm{SPOP}^{\mathrm{BTB}+}$ and $\mathrm{Gig}^{1-258 \triangle \mathrm{DDGS}}$.

\begin{tabular}{|c|c|c|c|}
\hline & $\begin{array}{l}\text { SPOP }^{\text {BTB+ }} \\
\text { (SeMet) }\end{array}$ & $\begin{array}{l}\text { Gig }^{1-258 \Delta \text { DDGS }} \\
\text { (SeMet) }\end{array}$ & $\begin{array}{l}\text { Gig }^{1-258 \Delta \text { DDGS }} \\
\text { (SeMet) }\end{array}$ \\
\hline \multicolumn{4}{|l|}{ DATA COLLECTION } \\
\hline Wavelength ( $\AA$ ) & 0.98 & 0.98 & 0.98 \\
\hline Space Group & $\mathrm{P} 1$ & $\mathrm{P} 21$ & $\mathrm{P} 21$ \\
\hline \multicolumn{4}{|l|}{ Cell Dimensions } \\
\hline $\mathrm{a}, \mathrm{b}, \mathrm{c}(\AA)$ & $36.8,88.7,88.7$ & $46.1,55.0,120.8$ & $46.5,55.6,120.6$ \\
\hline$\alpha, \beta, \gamma(\operatorname{deg})$ & $90.8,89.3,89.9$ & $90,91.0,90$ & $90,91.1,90$ \\
\hline Resolution ( $\AA$ ) & $20-2.5$ & $50-3.0$ & $100-2.8$ \\
\hline Total Reflections & 37,547 & 390,661 & 104,074 \\
\hline Unique Reflections & 37,547 & 12,742 & 15,314 \\
\hline Completeness ( $\%)$ & $96.2(79.7)$ & $91.1(59.3)$ & $91.0(59.1)$ \\
\hline Overall Rsym (\%) & $6.5(33.5)$ & $7.0(27.0)$ & $5.7(17.1)$ \\
\hline 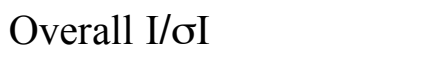 & $25.5(4.9)$ & $43(3.6)$ & $20.8(5.3)$ \\
\hline Mean Redundancy & $1.9(1.7)$ & $6.7(4.2)$ & $3.2(2.6)$ \\
\hline \multicolumn{4}{|l|}{ REFINEMENT } \\
\hline Rwork / Rfree & $0.220 / 0.259$ & & $0.294 / 0.360^{*}$ \\
\hline Reflections & 35675 & & 12179 \\
\hline Reflections (test set) & 1872 & & 615 \\
\hline Overall B-factors & 36.6 & & 54.7 \\
\hline \multicolumn{4}{|l|}{ R.M.S Deviations } \\
\hline Bond Lengths ( $\AA$ ) & 0.009 & & 0.03 \\
\hline Bond Angles ( deg ) & 1.2 & & 2.44 \\
\hline
\end{tabular}

* Structure of Gig ${ }^{1-258 \Delta D D G S}$ is still under refinement. 
which automatically performs density modification and non-crystallographic symmetry (NCS) averaging.

\subsubsection{Crystallographic Refinement of SPOP ${ }^{\mathrm{BTB}+}$}

Structure refinement of a macromolecule is an iterative process between model construction and comparison of model and structure factors. The aim is to build a structural model that best fits experimental data. R factor reflects the difference between the structure factors calculated from a model and those from the original X-ray diffraction data. $\mathrm{R}_{\text {free }}$ is the $\mathrm{R}$ factor calculated from a subset of reflections that are sequestered and not used for refinement. $\mathrm{R}_{\text {free }}$ monitors the refinement progress independently to prevent artifact model errors.

The initial structural model of $\mathrm{SPOP}^{\mathrm{BTB}+}$ was built de novo in the program $\mathrm{O}$ [130], with polyalanines based on the experimental map. Refinement procedures were initiated after backbones of $13 \alpha$ helices and $9 \beta$ strands were manually placed into the electron density. Five percent (1872 reflections) of all the independently measured reflections were randomly selected for the calculation of $\mathrm{R}_{\text {free. }}$ CNS (Crystallography \& NMR System) [131] software package was used to refine the structure. After the initial round of rigid body fitting refinement, $\mathrm{R}_{\text {work }} / \mathrm{R}_{\text {free }}$ dropped from 0.5 to 0.4 . The model was further simulated annealing refined by heating to $3000 \mathrm{~K}$ and gradual cooling $(25 \mathrm{~K}$ per cycle), and followed by overall temperature factor refinement (grouped, unrestrained B-factor refinement), which brought $\mathrm{R}_{\text {work }}$ and $\mathrm{R}_{\text {free }}$ down to under 0.4. The $2 \mathrm{Fo}-\mathrm{Fc}$ Fourier map was not as good as the experimental map at this point. Side chains were placed into the model based on the initial experimental map starting from the identified SeMet residues. A cycle of crystallographic conjugate gradient minimization refinement and restrained, individual B-factor refinement with CNS were performed after each cycle of placement of 5 to 10 residues or side chains. After 17 cycles of refinement carried out for the $\mathrm{SPOP}^{\mathrm{BTB}+}$ structure, the resulting $\mathrm{R}_{\text {work }}$ and $\mathrm{R}_{\text {free }}$ were 0.28 and 0.32 , respectively. $2 \mathrm{Fo}-\mathrm{Fc}$ and Fo-Fc Fourier maps were then used for further model construction. After additional 12 cycles of refinement, 220 water molecules were added to the structural model. The final cycle of refinement of SPOP ${ }^{\mathrm{BTB}}{ }^{+}$was performed including water molecules by minimization refinement and individual B-factor refinement, resulting in an $\mathrm{R}_{\text {work }}$ and $\mathrm{R}_{\text {free }}$ of 0.22 and 0.26 , respectively (Table 2.2). The Ramachandran plot was generated to check the main-chain torsion angles of SPOP ${ }^{\mathrm{BTB}+}$ structure model by program PROCHECK [132]. $92 \%$ residues were in the most favored region and none were in the disallowed region. There were two $\mathrm{SPOP}^{\mathrm{BTB}+}$ dimers in the asymmetric unit. The structure of each $\mathrm{SPOP}^{\mathrm{BTB}}{ }^{+}$dimer contained two chains, and each chain corresponding to one protomer of the dimer. The $\mathrm{SPOP}^{\mathrm{BTB}+}$ structure contained residues 174-231 and 237-331. Residues 172-173, 232-236, 332-337 were not observed in the electron density and were presumably disordered. 


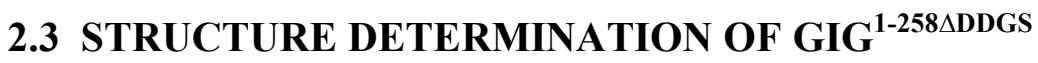

\subsubsection{Cloning, Engineering and Purification of Gig ${ }^{1-258 \triangle D D G S}$}

Using approaches described in section 2.1, to obtain sufficient protein for crystallization purposes, several Gigaxonin fragments were tested for expression and solubility. One Gig fragment comprising amino acids 1 to 258 crystallized. However, these crystals displayed no better diffraction than $8 \AA$. Multiple approaches were tried to improve crystal diffraction, including annealing by quick freezing and thawing, cross linking by incubating crystals with glutaraldehyde, and deleting potential flexible regions from expression constructs. Only the last method led to improved diffraction. Since we had determined the crystal structure of $\mathrm{SPOP}^{\mathrm{BTB}+}$, flexible regions (residues 235-ESKKNR-240) on the SPOP BTB domain were identified. Sequence alignment of SPOP and Gig indicated that the same region on Gig (residues 65-KDDGST-70) was

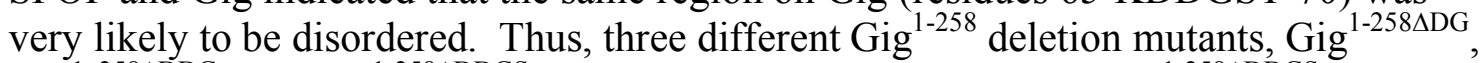
$\mathrm{Gig}^{1-258 \Delta \mathrm{DDG}}$, and Gig ${ }^{1-258 \Delta \mathrm{DDGS}}$, were made to optimize crystals. Gig ${ }^{1-258 \Delta \mathrm{DDGS}}$ crystallized in a different space group, and suitable diffraction data were obtained. Gig $^{1-258 \triangle D D G S}$ was therefore used for further study.

Gig $^{1-258 \Delta D D G S}$ was cloned into a derivative of the bacterial expression vector pGEX4T-1 generated by the Schulman's laboratory. Briefly, the thrombin cleavage sequence between the GST and the cloning site was swapped with the TEV (Tobacco etch virus protease) cleavage sequence. The vector containing the coding region for $\mathrm{Gig}^{1-258 \Delta \mathrm{DDGS}}$ was transformed into E.coli BL21 (DE3) GOLD. Expression of Gig $^{1-258 \triangle D D G S}$ was initiated through the inoculation of $200 \mathrm{ml}$ of L-Broth (LB) media containing a final concentration of $200 \mu \mathrm{g} / \mathrm{ml}$ ampicillin. $10 \mathrm{ml}$ of the cultured starter media was then used to inoculate each of the twelve 1-liter aliquots of LB media containing the final concentration of ampicillin of $200 \mu \mathrm{g} / \mathrm{ml}$. The induction, culture growing, and cell harvesting were performed in the way same as $\mathrm{SPOP}^{\mathrm{BTB}+}$ purification. Cell lysates from 12 liters of cultures were loaded onto a gravity column packed with 20 $\mathrm{ml}$ Glutathione Sepharose 4B resin with a flowthrough rate of approximately $1 \mathrm{ml}$ per minute. The resin was then washed with $100 \mathrm{ml}$ lysis buffer and the GST-Gig ${ }^{1-258 \Delta D D G S}$ was eluted with $50 \mathrm{mM}$ Tris (pH8.0), $200 \mathrm{mM} \mathrm{NaCl}, 10 \mathrm{mM}$ reduced Glutathione and 5 mM DTT. TEV was added to $\mathrm{Gig}^{1-258 \Delta \mathrm{DDGS}}$ to a mass ratio of 1:100. Cleavage proceeded at $4{ }^{\circ} \mathrm{C}$ overnight. The resulting GST and Gig ${ }^{1-258 \Delta D D G S}$ mixtures were diluted with $50 \mathrm{mM}$ Tris buffer containing no salt to make the final concentration of $\mathrm{NaCl}$ at 50 $\mathrm{mM}$. Diluted proteins were loaded onto a $30 \mathrm{ml}$ SOURCE Q column, using an AKTA FPLC system. GST and Gig ${ }^{1-258 \Delta D D G S}$ were eluted as two peaks with buffer containing 50 $\mathrm{mM}$ Tris $\mathrm{pH} 7.6,5 \mathrm{mM}$ DTT and 50 to $220 \mathrm{mM} \mathrm{NaCl}$ during gradient elution. The Gig $^{1-258 \Delta D D G S}$ protein was concentrated and further purified with SD200 gel filtration chromatography in buffer containing $20 \mathrm{mM}$ Tris $\mathrm{pH}$ 7.6, $150 \mathrm{mM} \mathrm{NaCl}$ and $5 \mathrm{mM}$ DTT, using an AKTA FPLC. The protein was flash frozen in liquid nitrogen and stored at -80 ${ }^{\circ} \mathrm{C}$. The SeMet labeled proteins were purified using the same methods. M9 media was used instead of LB media for culture; and $10 \mathrm{mM}$ DTT was added during purification for the SeMet protein where $5 \mathrm{mM}$ DTT was used for native protein purifications. 


\subsubsection{Crystallization of $\mathrm{Gig}^{1-258 \triangle \mathrm{DDGS}}$}

The initial crystallization screening was done using the hanging drop method over 9600 commercial conditions with wild type $\mathrm{Gig}^{1-258 \Delta \mathrm{DDGS}}$ protein at $4{ }^{\circ} \mathrm{C}$ and room temperature. Crystal drops were prepared by mixing $200 \mathrm{nl}$ of $25 \mathrm{mg} / \mathrm{ml}$ protein with 200 $\mathrm{nl}$ of precipitant solution, using the high throughput crystallization screening robot MOSQUITO. The drops were equilibrated by vapor diffusion against $100 \mu \mathrm{l}$ of precipitant solution. Initial crystals only grew at $4{ }^{\circ} \mathrm{C}$ in a condition containing $30 \%$ PEG 3350, 0.2 M KSCN, 5 mM DTT. The crystals were in a flower shape as a cluster of hundreds of tiny crystals. The quality of Gig ${ }^{1-258 \Delta \mathrm{DDGS}}$ crystals were greatly improved by manual streak seeding in $2 \mu \mathrm{l}$ room temperature drops containing $23 \%$ PEG 3350, $0.2 \mathrm{M}$ KSCN, 10 mM DTT, where crystals could not grow de novo. SeMet Gig ${ }^{1-258 \Delta D D G S}$ was used to obtain optimized crystals too. These optimized crystals had a sword-like shape with estimated dimensions of $300 \times 50 \times 50 \mu \mathrm{m}$. The crystals were harvested with supplemental $16 \%$ MPD and frozen by immersion in liquid nitrogen.

\subsubsection{Data Collection of Gig ${ }^{1-258 \triangle D D G S}$}

X-ray diffraction data sets were collected at the Advanced Light Source beamline 8.2.2. A fluorescence scan was performed prior to data collection to determine the peak energy/wavelength for Se. The data were collected at $\lambda=0.9804 \AA$ with an exposure time of 20 seconds per frame, and $0.5^{\circ}$ oscillation angles. The frames were recorded on an ADSC Q315 CCD detector. The unit cell parameters were determined using the HKL2000 software. The crystals belonged to the monoclinic space group P2(1) with unit cell dimension of $a=46.5 \AA, b=55.6 \AA, c=120.6 \AA, \beta=91.1^{\circ}$. Datasets from two crystals were collected: crystal 1 contains 720 frames (covering $360^{\circ}$ ) and crystal 2 contains 360 frames (covering $180^{\circ}$ ). A total of 390,661 reflections of crystal 1 were integrated and scaled with HKL2000 to a resolution of $3.0 \AA$. A total of 104,074 reflections of crystal 2 were integrated and scaled with HKL2000 to a resolution of $2.8 \AA$. Data processing parameters are summarized in Table 2.2.

\subsubsection{Phase Determination of Gig ${ }^{1-258 \Delta D D G S}$}

Single-wavelength anomalous dispersion (SAD) was used to determine the phase of Gig ${ }^{1-258 \Delta D D G S}$ from the data collected from crystal 1 [129]. The inputs for SOLVE included the space group, unit cell parameters, the scaled file from HKL2000 [127], wavelength of $0.9804 \AA$, f' of -7.6, f "of 6.4 (f' and f" were calculated from the fluorescence scan of the SeMet labeled Gig ${ }^{1-258 \Delta D D G S}$ crystal), and the number of expected anomalous atoms. Since each Gig molecule contains six SeMets and an estimated two molecules in the asymmetric unit, we set up the SOLVE program to search for $20 \mathrm{Se}$ atoms, which includes an additional 8 atoms in case the program picks up wrong sites. 10 out of 12 expected Se sites were found by SOLVE with occupancy scores better than 0.05 . The phases were further improved with the software RESOLVE, which automatically performs density modification and NCS averaging. We were not 
able to determine the phase from data collected from crystal 2, probably due to weak anomalous signals.

\subsubsection{Crystallographic Refinement of Gig ${ }^{1-258 \Delta D D G S}$}

From the experimental map obtained from RESOLVE, we were able to place the alpha carbon backbone of Gig ${ }^{1-258 \Delta D D G S}$ into the electron density with the program $\mathrm{O}$ [130]. Since data collected from crystal 2 has a slightly higher resolution than these collected from crystal 1 , further refinement of the structural model was performed using the data collected from crystal 2. Five percent of all the independently measured reflections were randomly selected for the calculation of $\mathrm{R}_{\text {free. }}$ The CNS

(Crystallography \& NMR System) [131] software package was used to refine the structure. The model was first subjected to simulated annealing, refined by heating to $3000 \mathrm{~K}$ and gradual cooling ( $25 \mathrm{~K}$ per cycle), and followed by overall temperature factor refinement (grouped, unrestrained B-factor refinement). The 2Fo-Fc Fourier map was used to construct the structural model. The anomalous signals from SeMet side chains and sequence alignment of $\mathrm{SPOP}^{\mathrm{BTB}+}$ and $\mathrm{Gig}^{1-258 \triangle \mathrm{DDGS}}$ helped to correlate the sequence of residue side chains with placement in the electron density map. A cycle of crystallographic minimization refinement and individual B-factor refinement with CNS were performed after each cycle of placement of 5 to 10 residues or side chains. More than 30 cycles of refinement were carried out on $\mathrm{Gig}^{1-258 \Delta \mathrm{DDGS}}$, resulting in an $\mathrm{R}_{\mathrm{work}}$ and $\mathrm{R}_{\text {free }}$ of 0.29 and 0.32 , respectively. It is difficult to further refine the structure due to the poor electron density over regions other than the Gig BTB domain. There is only one Gig $^{1-258 \Delta D D G S}$ dimer in the asymmetric unit. The Gig ${ }^{1-258 \Delta D D G S}$ dimer contains two chains, chain $\mathrm{A}$ and chain $\mathrm{B}$, with each chain corresponding to one protomer of the dimer.

Molecule A contains residues 6-59, 72-161, which comprise the BTB core and the 3-box. The electron densities over residues 1-5 and residues 60-71 (without residue 66-69

DDGS) are too poor for structure building. Molecule A also contains additional 80 amino acids that are mainly built with poly-alanines due to poor electron density over the region C terminal to the 3-box. Sequence "HM", "MSAL", and "LREQML" were assigned based on anomalous signals obtained from SeMet. Molecule B contains the same residue composition as molecule A in the BTB core and the 3-box, and additional 68 non-continuous residues built with poly alanines with regions containing "HMKDVMSALWVSG" and "SSYLREQMLNEP" side-chains assigned, and confirmed based on locations of SeMet. In both molecules, the BTB domain and the 3-box (residues 1 to 161) were ambiguously assigned with decent experimental maps and simulated component omit maps. In the structure analysis in Chapter 4, the structure of residues 162-258 was omitted for clarity. 


\subsection{STRUCTURE DETERMINATION OF SPOP ${ }^{\mathrm{MATH}}{ }_{-S B C}$ COMPLEXES}

\subsubsection{Cloning and Purification of SPOP}

The SPOP MATH domain contains residues 28-166. It was cloned into the bacterial expression vector pGEX4T-1 derivative described above, where the thrombin cleavage sequence between the GST and the cloning site was swapped with the TEV (Tobacco etch virus protease) cleavage sequence. The vector containing the coding region for SPOP ${ }^{\mathrm{MATH}}$ was transformed into E.coli BL21 (DE3) GOLD. Expression of SPOP $^{\mathrm{MATH}}$ was initiated through the inoculation of $200 \mathrm{ml}$ of L-Broth (LB) media containing a final concentration of $200 \mu \mathrm{g} / \mathrm{ml}$ ampicillin. $10 \mathrm{ml}$ of the cultured starter media was then used to inoculate each of the twelve 1-liter aliquots of LB media containing the final concentration of ampicillin of $200 \mu \mathrm{g} / \mathrm{ml}$. The 1-liter cultures were allowed to grow at $37^{\circ} \mathrm{C}$ at $200 \mathrm{rpm}$ for five to six hours yielding an $\mathrm{OD}_{600}$ of 0.8 to 0.9 . Induction of T7 RNA polymerase was done through the addition of IPTG to a final concentration of $0.6 \mathrm{mM}$. The cultures were then incubated at $24^{\circ} \mathrm{C}$ in a rotary shaker overnight (15 to 18 hours) to allow protein expression. Cell lysates from 12 liters of cultures were loaded on a gravity column packed with $20 \mathrm{ml}$ Glutathione Sepharose 4B resin with an approximate flowthrough rate of $1 \mathrm{ml}$ per minute. The resin was then washed with $100 \mathrm{ml}$ lysis buffer and the GST- SPOP ${ }^{\mathrm{MATH}}$ was eluted with $50 \mathrm{mM}$ Tris pH8.0, $200 \mathrm{mM} \mathrm{NaCl}, 10 \mathrm{mM}$ reduced Glutathione and $5 \mathrm{mM}$ DTT. TEV was added to the eluted protein to a mass ratio of $1: 100$. Cleavage proceeded at $4{ }^{\circ} \mathrm{C}$ overnight. The resulting GST and SPOP ${ }^{\mathrm{MATH}}$ mixture was diluted with $50 \mathrm{mM}$ HEPES (4-(2-hydroxyethyl)-1-piperazineethanesulfonic acid) buffer containing no salt to make the final concentration of $\mathrm{NaCl}$ at $50 \mathrm{mM}$. Due to the basic $\mathrm{pI}$ of the SPOP MATH domain, the diluted proteins were loaded onto a $30 \mathrm{ml}$ SOURCE S column, using the AKTA FPLC system. GST and SPOP ${ }^{\mathrm{MATH}}$ were eluted as two peaks with buffer containing $50 \mathrm{mM}$ HEPES pH 7.0, $5 \mathrm{mM}$ DTT and 50 to $220 \mathrm{mM} \mathrm{NaCl}$ during gradient elution. The SPOP ${ }^{\mathrm{MATH}}$ protein was concentrated and further purified with SD200 gel filtration chromatography in buffer containing $20 \mathrm{mM}$ Tris $\mathrm{pH} 7.6,150 \mathrm{mM} \mathrm{NaCl}$ and 5 $\mathrm{mM}$ DTT, using an AKTA FPLC. The protein with the concentration of $50 \mathrm{mg} / \mathrm{ml}$ was flash frozen in liquid nitrogen and stored at $-80{ }^{\circ} \mathrm{C}$.

\subsubsection{Crystallization of SPOP ${ }^{\mathrm{MATH}}-\mathrm{SBC}$ Complexes}

Peptides containing SPOP binding consensus (SBC) were dissolved in water to make a stock of $100 \mathrm{mM}$ peptides. SPOP ${ }^{\mathrm{MATH}}$ and each SBC peptide were mixed at 1:2.5 molar ratios and incubated on ice for 1 to 2 hours before setting up crystallization trials. Initial crystallization screening was done using the hanging drop method over 9600 commercial conditions for each SPOP ${ }^{\mathrm{MATH}}$-SBC mixture, at room temperature. Crystallization trial drops were prepared by mixing $200 \mathrm{nl}$ of protein with $200 \mathrm{nl}$ of precipitant solution, using the high throughput crystallization screening robot MOSQUITO. SPOP ${ }^{\mathrm{MATH}}$ crystallized with four different peptides: MacroH2A ${ }^{\mathrm{SBC}}$ pep1, MacroH2A ${ }^{\mathrm{SBC}}$ pep2, $\mathrm{Puc}^{\mathrm{SBC} 1}$, and $\mathrm{Ci}^{\mathrm{SBC} 2}$. $\mathrm{SPOP}^{\mathrm{MATH}}-\mathrm{Puc}^{\mathrm{SBC} 1}, \mathrm{SPOP}^{\mathrm{MATH}}-\mathrm{Ci}^{\mathrm{SBC} 2}$ and 
$\mathrm{SPOP}^{\mathrm{MATH}}$-MacroH2A ${ }^{\mathrm{SBC}}$ pep2 crystals grew overnight at room temperature and were optimized by manual streak seeding with reservoir solution containing 14-16 \% PEG (polyethylene glycol) 2000, $0.2 \mathrm{M}$ ammonium sulfate, $0.05 \mathrm{M}$ sodium acetate, $\mathrm{pH}$ 4.6. $20 \%$ glycerol was used as cryoprotectant for harvesting. $\mathrm{SPOP}^{\mathrm{MATH}}$ - MacroH2 ${ }^{\mathrm{SBC}}$ pep1 crystals grew at $18{ }^{\circ} \mathrm{C}$ in $12 \%$ PEG 550, $0.1 \mathrm{M}$ 2-(N-morpholino) ethanesulfonic acid (MES), $20 \mathrm{mM} \mathrm{ZnSO}_{4}$, $\mathrm{pH} 6.5$ one week after drop set up and were harvested with an additional $30 \%$ PEG 400.

\subsubsection{Data Collection of SPOP ${ }^{\mathrm{MATH}}{ }_{-}$SBC Complexes}

Diffraction data were collected remotely from St. Jude, using Advanced Photon Source SERCAT (Southeast Regional Collaborative Access Team) Sector 22 ID and BM beamlines. The $\mathrm{SPOP}^{\mathrm{MATH}}$-MacroH $2 \mathrm{~A}^{\mathrm{SBC}}$ pep1 data were collected at $\lambda=1.0 \AA$ with an exposure time of 3 seconds per frame, and $0.2^{\circ}$ oscillation angles. 500 frames were recorded. The unit cell parameters were determined using the HKL2000 software. The crystals belonged to the hexagonal space group P6(5)22 with unit cell dimensions of $\mathrm{a}=\mathrm{b}=44.7 \AA, \mathrm{c}=268.0 \AA$. A total of 333,421 reflections were integrated and scaled with HKL2000 to a resolution of $2.3 \AA$. The SPOP ${ }^{\mathrm{MATH}}{ }_{-}$-MacroH2 $\mathrm{A}^{\mathrm{SBC}}$ pep2 data were collected at $\lambda=1.0 \AA$ with an exposure time of 2 seconds per frame, and $0.7^{\circ}$ oscillation angles. 300 frames were recorded. The crystals belonged to the monoclinic space group $\mathrm{C} 2$ with unit cell dimension of $\mathrm{a}=89.0 \AA, \mathrm{b}=43.1 \AA, \mathrm{c}=87.5 \AA, \beta=118.1^{\circ}$. A total of 450,833 reflections were integrated and scaled with HKL2000 to a resolution of $1.43 \AA$. The SPOP ${ }^{\mathrm{MATH}}{ }_{-} \mathrm{Puc}^{\mathrm{SBC} 1}$ data were collected at $\lambda=1.0 \AA$ with an exposure time of 5 second per frame, and $0.5^{\circ}$ oscillation angles. 280 frames were recorded. The crystals belonged to the monoclinic space group $\mathrm{C} 2$ with unit cell dimension of $\mathrm{a}=90.8, \mathrm{~b}=43.7$, $\mathrm{c}=86.8 \AA, \beta=107.0^{\circ}$. The total 362,270 reflections were integrated and scaled with HKL2000 to a resolution of $1.66 \AA$. The $\mathrm{SPOP}^{\mathrm{MATH}}-\mathrm{Ci}^{\mathrm{SBC} 2}$ data were collected at $\lambda=1.0 \AA$ with an exposure time of 3 second per frame, and $0.5^{\circ}$ oscillation angles. 400 frames were recorded. The crystals belonged to the triclinic space group P1 with unit cell dimension of $\mathrm{a}=44.3 \AA, \mathrm{b}=48.1 \AA, \mathrm{c}=49.9 \AA, \alpha=63.0^{\circ}, \beta=64.0^{\circ}, \gamma=62.9^{\circ}$. A total of 31,963 reflections were integrated and scaled with HKL2000 to a resolution of $1.74 \AA$. Data processing parameters are summarized in Table 2.3.

\subsubsection{Phase Determination of SPOP ${ }^{\mathrm{MATH}}$-SBC Complexes}

Molecular replacement (MR) is a method to determine the phase for X-ray crystallography. MR depends on the use of structures homologous to the structure from which the diffraction data is collected. Since the solution structure of SPOP MATH domain was available in the Protein Data Bank due to structural genomics efforts (2cr2.pdb), molecular replacement was used to determine the initial phases for each complex structure. We edited the NMR (Nuclear magnetic resonance) structure to generate the model for molecular replacement. First, four conformations were randomly selected from twenty conformations of the NMR structure; then the flexible regions of each structure were deleted and the remaining residues were mutated to Ala; finally the 
Table 2.3. Structure data and refinement statistics of SPOP ${ }^{\mathrm{MATH}}-\mathrm{SBC}$.

\begin{tabular}{|c|c|c|c|c|}
\hline & $\begin{array}{l}\text { SPOP }^{\text {MATH }} \\
\text { MH2A }^{\text {SBC }_{1}}\end{array}$ & $\begin{array}{l}\text { SPOP }^{\text {MATH }} \\
\text { MH2A }^{\text {SBC }_{2}} \\
\end{array}$ & $\begin{array}{c}\text { SPOP }^{\mathrm{MATH}} \\
\mathrm{Ci}^{\mathrm{SBC2}} \\
\end{array}$ & $\begin{array}{c}\text { SPOP }^{\text {MATH }} \\
\text { Puc }^{\text {SBC1 }}\end{array}$ \\
\hline \multicolumn{5}{|l|}{$\begin{array}{l}\text { DATA } \\
\text { COLLECTION }\end{array}$} \\
\hline Wavelength ( $\AA$ ) & 1 & 1 & 1 & 1 \\
\hline Space Group & P6522 & $\mathrm{C} 2$ & $\mathrm{P} 1$ & $\mathrm{C} 2$ \\
\hline \multicolumn{5}{|l|}{ Cell Dimensions } \\
\hline $\mathrm{a}, \mathrm{b}, \mathrm{c}(\AA)$ & $44.7,44.7,268.0$ & $89.0,43.1,87.5$ & $44.3,48.1,49.9$ & $90.8,43.7,86.8$ \\
\hline$\alpha, \beta, \gamma(\operatorname{deg})$ & $90,90,120$ & $90,118.1,90$ & $63,64.0,62.9$ & $90,107.0,90$ \\
\hline Resolution ( $\AA$ ) & $50-2.29$ & $50-1.43$ & $50-1.74$ & $20-1.66$ \\
\hline Total Reflections & 333,421 & 450,833 & 31963 & 362,270 \\
\hline Unique Reflections & 8,052 & 54,191 & 985 & 38,876 \\
\hline Completeness $(\%)$ & $98.9(96.8)$ & $99.2(97.7)$ & $97(94.6)$ & $99.5(99.0)$ \\
\hline Overall Rsym (\%) & $9.0(18.2)$ & $5.7(11.0)$ & $3.1(10.2)$ & $7.3(39.0)$ \\
\hline Overall I/ol & $31.7(8.3)$ & $31.7(14.8)$ & $30.3(9.5)$ & $28.9(3.1)$ \\
\hline Mean Redundancy & $7.0(4.5)$ & $4.3(4.2)$ & $2.0(1.9)$ & $3.1(2.8)$ \\
\hline \multicolumn{5}{|l|}{ REFINEMENT } \\
\hline Rwork / Rfree & $0.258 / 0.275$ & $0.193 / 0.230$ & $0.175 / 0.215$ & $0.248 / 0.258$ \\
\hline Reflections & 7,132 & 51019 & 29424 & 36636 \\
\hline Reflections (test set) & 362 & 2739 & 1582 & 1934 \\
\hline Overall B-factors & 41 & 17.5 & 13.2 & 22.2 \\
\hline \multicolumn{5}{|l|}{ R.M.S Deviations } \\
\hline Bond Lengths $(\AA)$ & 0.009 & 0.008 & 0.01 & 0.009 \\
\hline Bond Angles (deg) & 1.16 & 1.16 & 1.26 & 1.19 \\
\hline \multicolumn{5}{|l|}{ Ramachandran Plots } \\
\hline Most Favored & $97.56 \%$ & $97.56 \%$ & $98.45 \%$ & $98.05 \%$ \\
\hline Additional & $2.44 \%$ & $2.44 \%$ & $1.57 \%$ & $1.95 \%$ \\
\hline Disallowed & 0 & 0 & 0 & 0 \\
\hline
\end{tabular}


B-factors were assigned to a value of $20 \AA^{2}$. MR was first performed with the dataset collected from SPOP ${ }^{\mathrm{MATH}}$-MacroH2 ${ }^{\mathrm{SBC}}$ pep1 crystal. The estimated molecule number in the asymmetric unit is one for this space group, which is less complex for MR. Four conformations were used as an ensemble in software PHASER [133] to search for solutions. The solution was confirmed by the high quality of a simulated annealing composite omit map. The structure of SPOP ${ }^{\mathrm{MATH}}-\mathrm{MacroH} 2 \mathrm{~A}^{\mathrm{SBC}}$ pep1 was refined and the SPOP ${ }^{\mathrm{MATH}}$ structure without the SBC peptide was used for MR in other datasets. For other three SPOP ${ }^{\mathrm{MATH}}$ - SBC structures, the estimated number of molecules in the asymmetric unit is two. PHASER successfully found the solution for $\mathrm{SPOP}^{\mathrm{MATH}}-\mathrm{MacroH} 2 \mathrm{~A}^{\mathrm{SBC}}$ pep2 and $\mathrm{SPOP}^{\mathrm{MATH}}-\mathrm{Ci}^{\mathrm{SBC} 2}$ but failed to find the solution for $\mathrm{SPOP}^{\mathrm{MATH}}{ }_{-\mathrm{Puc}}{ }^{\mathrm{SBC} 1}$. A translational pseudo symmetry operator $(0.0,0.5,0.5)$ was identified by program PHENIX.xtriage, which potentially caused the failure of automatic MR by PHASER. A step-by-step molecular replacement by program MolRep was performed to determine the initial phase of SPOP ${ }^{\mathrm{MATH}}-\mathrm{Puc}^{\mathrm{SBC} 1}$. First, a cross rotation function was performed, using the SPOP ${ }^{\mathrm{MATH}}$ from $\mathrm{SPOP}^{\mathrm{MATH}}-\mathrm{MacroH} 2 \mathrm{~A}^{\mathrm{SBC}}$ as the model. This resulted in several solutions. The translation functions, using the best rotation function peak result $\left(\alpha=225.3^{\circ}, \beta=0.0^{\circ}, \gamma=135.3^{\circ}\right)$, were performed and resulted in the best solution with translational operator $(0.083,0.0,0.283)$. This defined the position of one MATH domain molecule. The position of the second MATH molecule was calculated by applying the pseudo-translational operator to the first molecule. This solution was confirmed by a simulated annealing composite omit map.

\subsubsection{Crystallographic Refinement of SPOP ${ }^{\mathrm{MATH}}-\mathrm{SBC}$ Complexes}

Since the resolution of the SPOP ${ }^{\mathrm{MATH}}-\mathrm{MacroH} 2 \mathrm{~A}^{\mathrm{SBC}}$ pep2 dataset was high (1.43 $\AA$ ), automatic construction and refinement were performed with ARP/wARP [134].The resulting model contains an ARP/wARP constructed SPOP MATH domain with side chains, giving rise to refinement statistics of $R_{w o r k}$ and $R_{\text {free }}$ of 0.28 and 0.31 , respectively. 2Fo-Fc and Fo-Fc maps generated from this model clearly showed extra continuous electron density around the $\mathrm{SPOP}^{\mathrm{MATH}}$ substrate binding site. MacroH $2 \mathrm{~A}^{\mathrm{SBC}}$ pep2 residues and additional residues on $\mathrm{SPOP}^{\mathrm{MATH}}$ were manually placed in the program COOT. The structure underwent cycles of refinement with the program Refmac [135], which optimizes the structure coordinate parameters to satisfy maximum likelihood. Structures of other SPOP ${ }^{\mathrm{MATH}}$-SBC complexes were also constructed in COOT [136] and refined with Refmac [135]. Detailed refinement parameters are summarized in Table 2.3. The residues that were not modeled in the following structures were not clear in the electron density and were presumably disordered.

$S P O P^{M A T H}-$ MacroH2A $A^{S B C}$ pep 1: The structure of SPOP ${ }^{\mathrm{MATH}}-\mathrm{MacroH} 2 \mathrm{~A}^{\mathrm{SBC}}$ pep1 contained SPOP $^{\mathrm{MATH}}$ residues 28-59, 64-143, 146-165, and MacroH2A residues 169-175. $\mathrm{SPOP}^{\mathrm{MATH}}$ residues $60-63,144-145$ and MacroH2A residues 166-168, 176-180 were not observed and side chains of SPOP ${ }^{\mathrm{MATH}}$ residues $28,64,78-81,143,165$ and MacroH2A residue 175 were not modeled. The structure contained a single zinc atom from the crystallization solution, mediating inter-molecular crystal contacts. 
SPOP $P^{M A T H}$-MacroH2 $A^{S B C}$ pep2: There were two SPOP ${ }^{\mathrm{MATH}}$ and one peptide in the asymmetric unit. The structure contained $\mathrm{SPOP}^{\mathrm{MATH}}$ residues $28-59,64-77,80-164$, and MacroH2A residues 171-181. SPOP ${ }^{\mathrm{MATH}}$ residues 60-63, 78-79, 165-166 and MacroH2A residues 182-185 were not observed and side chains of SPOP ${ }^{\mathrm{MATH}}$ residues 119-121, 145-146 and 164 were not modeled. The structure contained two molecules of ammonium sulfate from the crystallization solution, mediating inter-molecular crystal contacts.

$S P O P^{M A T H}-P u c^{S B C 1}$ : There were two SPOP ${ }^{\mathrm{MATH}}$ and two $\mathrm{Puc}^{\mathrm{SBC} 1}$ peptides in the asymmetric unit. The structure contained $\mathrm{SPOP}^{\mathrm{MATH}}$ residues 28-59, 64-164, and Puc residues 96-102. SPOP ${ }^{\mathrm{MATH}}$ residues 60-63, 165-166 and MacroH2A residues 93-95, 103-107 were not observed and side chains of SPOP ${ }^{\mathrm{MATH}}$ residues 64-65, 94-97, 110-112 and Puc residues 96-97 were not modeled. The structure also contained two molecules of ammonium sulfate from the crystallization solution, mediating inter-molecular crystal contacts.

$S P O P^{M A T H}-C i{ }^{S B C 2}$ : There were two $\mathrm{SPOP}^{\mathrm{MATH}}$ and two $\mathrm{Ci}^{\mathrm{SBC} 2}$ peptides in the asymmetric unit. The structure contained $\mathrm{SPOP}^{\mathrm{MATH}}$ residues $29-59,63-164$, and $\mathrm{Ci}$ residues 1360-1366. SPOP ${ }^{\mathrm{MATH}}$ residues 60-62, 165-166 and Ci residues 1357-1359, 1367-1371 were not observed and side chains of $\mathrm{SPOP}^{\mathrm{MATH}}$ residues 63-65, 95 and $\mathrm{Ci}$ residue 1361 were not modeled.

\subsection{STRUCTURE DETERMINATION OF SPOP MATH-BTB $+^{\mathrm{MAU}}$ UC $^{\mathrm{SBC} 1}$}

\subsubsection{Cloning and Purification of SPOP ${ }^{\mathrm{MATH}-\mathrm{BTB}+}$}

Various SPOP constructs were made with different expression vectors. We were able to purify a SPOP fragment containing residues 28 to 337 , which was referred to as SPOP $^{\text {MATH-BTB }+}$. SPOP ${ }^{\mathrm{MATH}-\mathrm{BTB}+}$ was fused to the $\mathrm{C}$ terminus of His tagged Maltose-binding protein (His-MBP) and cloned into the first cloning site of the pRSFDuet vector. A TEV cleavage site was engineered between His-MBP and $\mathrm{SPOP}^{\mathrm{MATH}-\mathrm{BTB}+}$. The vector was transformed into E.coli BL21 (DE3) GOLD. Expression was initiated through the inoculation of $200 \mathrm{ml}$ of L-Broth (LB) media containing a final concentration of $50 \mu \mathrm{g} / \mathrm{ml}$ kanamycin. $10 \mathrm{ml}$ of the cultured starter media was then used to inoculate each of the twelve 1-liter aliquots of LB media containing the final concentration of kanamycin of $50 \mu \mathrm{g} / \mathrm{ml}$. The 1-liter cultures were allowed to grow at $37^{\circ} \mathrm{C}$ at $200 \mathrm{rpm}$ for five to six hours yielding an $\mathrm{OD}_{600}$ of 0.8 to 0.9 . Induction of T7 RNA polymerase was done through the addition of IPTG to a final concentration of $0.6 \mathrm{mM}$. The cultures were then incubated at $16{ }^{\circ} \mathrm{C}$ in a rotary shaker overnight (15 to 18 hours) to allow protein expression. Cell lysates from 12 liters of cultures were loaded on a gravity column packed with $20 \mathrm{ml}$ nickel-NTA resin with an approximate flow through rate of $1 \mathrm{ml}$ per minute. The resins were then washed with 100 $\mathrm{ml}$ PBS (Phosphate buffered saline) buffer and the His-MBP-SPOP ${ }^{\mathrm{MATH}-\mathrm{BTB}^{+}}$was eluted 
with PBS pH 7.5 containing $250 \mathrm{mM}$ imidazole and $2 \mathrm{mM}$ BME ( $\beta$-mercaptoethanol). TEV was added to the eluted protein to a mass ratio of 1:100. Cleavages proceeded at $4{ }^{\circ} \mathrm{C}$ overnight. His-MBP and SPOP ${ }^{\mathrm{MATH}-\mathrm{BTB}^{+}}$mixture were diluted with $50 \mathrm{mM}$ Tris buffer ( $\mathrm{pH}$ 7.6) containing no salts to make the final concentration of sodium chloride at $50 \mathrm{mM}$. Diluted proteins were loaded on a $30 \mathrm{ml}$ SOURCE Q column, using an AKTA FPLC system. His-MBP and SPOP ${ }^{\mathrm{MATH}-\mathrm{BTB}^{+}}$were eluted as two peaks with buffer containing $50 \mathrm{mM}$ Tris $\mathrm{pH}$ 7.6, $5 \mathrm{mM}$ DTT and 50 to $220 \mathrm{mM} \mathrm{NaCl}$ during gradient elution. SPOP ${ }^{\mathrm{MATH}-\mathrm{BTB}+}$ was concentrated, further purified with SD200 gel filtration chromatography in buffer containing $20 \mathrm{mM}$ Tris $\mathrm{pH} 7.6,150 \mathrm{mM} \mathrm{NaCl}$ and $5 \mathrm{mM}$ DTT, using an AKTA FPLC, aliquotted, flash frozen in liquid nitrogen and stored at $-80{ }^{\circ} \mathrm{C}$.

\subsubsection{Crystallization of SPOP ${ }^{\mathrm{MATH}-\mathrm{BTB}+}-\mathrm{Puc}^{\mathrm{SBC} 1}$}

$\mathrm{SPOP}^{\mathrm{MATH}-\mathrm{BTB}+}$ and $\mathrm{Puc}^{\mathrm{SBC} 1}$ peptide were mixed at a 1:5 molar ratio and incubated on ice for 1 to 2 hours before setting up drops. Initial crystallization screening was done using the hanging drop method over 9600 conditions at $4{ }^{\circ} \mathrm{C}$. Crystallization trial drops were prepared by mixing $200 \mathrm{nl}$ of protein with $200 \mathrm{nl}$ of precipitant solution, using the high throughput crystallization screening robot MOSQUITO. Crystals grew in 15-20 \% PEG 3350, 0.1 M LiCl, 0.1 M Bis-Tris, pH 5.5 in about one week. These crystals were single but tiny, with thousands of crystals in a drop. Optimized crystals grew at $4{ }^{\circ} \mathrm{C}$ two weeks after manually streak seeding initial crystals in $10 \%$ PEG 3350, $0.1 \mathrm{M} \mathrm{LiCl}, 0.05 \mathrm{M}$ Bis-Tris, $\mathrm{pH}$ 5.5. The optimized crystals had rod-like shapes and approximate dimensions of $70 \times 50 \times 50 \mu \mathrm{m}$. SPOP $^{\mathrm{MATH}-\mathrm{BTB}+}-\mathrm{Puc}^{\mathrm{SBC} 1}$ crystals were harvested in crystallization solution supplemented with $30 \%$ MPD.

\subsubsection{Data Collection of SPOP ${ }^{\mathrm{MATH}-\mathrm{BTB}+}-\mathrm{Puc}^{\mathrm{SBC} 1}$}

X-ray diffraction data sets were collected at the Advanced Photon Source NECAT (the Northeastern Collaborative Access Team) Sector 24 ID-C. Two datasets were collected from two crystals, crystal 1 and crystal 2 . The data of crystal 1 were collected at $\lambda=1.0 \AA$ with an exposure time of 1 second per frame, and $1.0^{\circ}$ oscillation angles. 110 frames were recorded. The crystal belonged to the orthorhombic space group P2(1)22 with unit cell dimensions of $\mathrm{a}=55.3 \AA, \mathrm{b}=106.8 \AA, \mathrm{c}=130.5 \AA$. A total of 215,492 reflections were integrated and scaled with HKL2000 to a resolution of $2.6 \AA$. The data of crystal 2 were collected at the same condition as that for crystal 1. 180 frames were recorded. The crystal belonged to the orthorhombic space group P2(1)22 with unit cell dimensions of $\mathrm{a}=63.5 \AA, \mathrm{b}=107.5 \AA, \mathrm{c}=130.7 \AA$. A total of 543,183 reflections were integrated and scaled with HKL2000 to a resolution of $2.7 \AA$. Data processing parameters are summarized in Table 2.4. 
Table 2.4. Structure data and refinement statistics of $\mathrm{SPOP}^{\mathrm{MATH}-\mathrm{BTB}+}-\mathrm{Puc}^{\mathrm{SBC} 1}$.

\begin{tabular}{|c|c|c|}
\hline & $\begin{array}{l}\text { SPOP } \\
\text { (Crystal1) }\end{array}$ & $\begin{array}{l}\text { SPOP } \\
\text { (Crystal2) }\end{array}$ \\
\hline \multicolumn{3}{|l|}{ DATA COLLECTION } \\
\hline Wavelength ( $\AA$ ) & 1 & 1 \\
\hline Space Group & P212121 & P212121 \\
\hline \multicolumn{3}{|l|}{ Cell Dimensions } \\
\hline $\mathrm{a}, \mathrm{b}, \mathrm{c}(\AA)$ & $55.3,106.8,130.5$ & $63.5,107.5,130.7$ \\
\hline$\alpha, \beta, \gamma(\operatorname{deg})$ & $90,90,90$ & $90,90,90$ \\
\hline Resolution ( $\AA$ ) & $50-2.62$ & $50-2.7$ \\
\hline Total Reflections & 215,492 & 543,183 \\
\hline Unique Reflections & 24,800 & 25,440 \\
\hline Completeness (\%) & 98.1(91.3) & $94.5(75.1)$ \\
\hline Overall Rsym (\%) & $10.6(29.6)$ & $17.4(59.0)$ \\
\hline Overall I/oI & $20.0(3.7)$ & $20.1(2.4)$ \\
\hline Mean Redundancy & $4.6(3.8)$ & $9.4(5.9)$ \\
\hline \multicolumn{3}{|l|}{ REFINEMENT } \\
\hline Rwork / Rfree & $0.230 / 0.285$ & $0.256 / 0.318$ \\
\hline Reflections (working set) & 21811 & 21315 \\
\hline Reflections (test set) & 1235 & 1211 \\
\hline Overall B-factors & 35 & 57.8 \\
\hline \multicolumn{3}{|l|}{ R.M.S Deviations } \\
\hline Bond Lengths ( $\AA$ ) & 0.009 & 0.01 \\
\hline Bond Angles (deg) & 1.19 & 1.29 \\
\hline \multicolumn{3}{|l|}{ Ramachandran Statistics } \\
\hline Most Favored & $95.39 \%$ & $85.30 \%$ \\
\hline Additional Allowed & $4.43 \%$ & $14.70 \%$ \\
\hline Disallowed & 0 & 0 \\
\hline
\end{tabular}




\subsubsection{Structure Determination of SPOP ${ }^{\mathrm{MATH}-\mathrm{BTB}+}-\mathrm{Puc}^{\mathrm{SBC} 1}$}

Molecular replacement (MR) was used to determine the structure solution for both crystals. The program PHASER [133] was used to perform automatic MR. Two ensembles, one containing SPOP ${ }^{\mathrm{MATH}}$ structures and another containing $\mathrm{SPOP}^{\mathrm{BTB}+}$ monomer, were used as search models. The solution was validated by the formation of the $\mathrm{SPOP}^{\mathrm{BTB}+}$ dimer structure after searching with only one protomer of the BTB domain. The structures were built in COOT [136] and refined with Refmac [135] (Table 2.4).

$S P O P^{M A T H-B T B+}-P u c^{S B C 1}$ (crystal 1): The asymmetric unit contained one dimeric $\mathrm{SPOP}^{\mathrm{MATH}-\mathrm{BTB}+}$ and two associated Puc ${ }^{\mathrm{SBC} 1}$ peptides. The structure contained SPOP molecule A residues 29-59, 64-231, 237-331, SPOP molecular B residues 29-59, 64-167, 174-231, 237-331 and Puc residues 97-102 in both peptides. SPOP residues 60-63, 232-236, 332-337 and Puc residues were not modeled due to poor electron density. SPOP residues 168-173 (the linker between MATH domain and BTB domain) were modeled with poly-alanines in molecule A but not built in molecule B. Those residues were presumably disordered.

$S P O P^{M A T H-B T B+}-P u c^{S B C l}$ (crystal 2): The asymmetric unit contained one dimeric $\mathrm{SPOP}^{\mathrm{MATH}-\mathrm{BTB}+}$ and two associated Puc ${ }^{\mathrm{SBC} 1}$ peptides. The structure contained SPOP molecule A residues 29-59, 64-167, 174-231, 237-331, SPOP molecule B residues 29-59, 64-167, 177-231, 237-330 and Puc residues 97-102 in both peptides. SPOP residues $60-63,232-236,332-337$ were not modeled in both molecules. SPOP residues 168-176, which are the linker between MATH domain and BTB domain were observed in molecule B and partly modeled (residues 174-176) in molecule A. Those residues were presumably disordered.

\subsection{IDENTIFICATION OF THE SPOP BINDING CONSENSUS (SBC)}

\subsubsection{Identification of a SPOP Binding Peptide in Puc}

For the purpose of crystallographic studies, we managed to purify a protein complex of SPOP ${ }^{\mathrm{MATH}}$ and Puc ${ }^{1-390}$ (Table 2.1). However, this complex did not yield any crystals. One way to obtain crystals is to find a minimally folded regions. As folded regions are often resistant to cleavage by nonspecific proteases, we used this $\mathrm{SPOP}^{\mathrm{MATH}}-\mathrm{Puc}^{1-390}$ complex for limited proteolysis analysis. The initial aim was to identify a minimal stable SPOP-Puc complex that may yield crystals. SPOP ${ }^{\mathrm{MATH}}$ was purified as described above. Puc ${ }^{1-390}$ was cloned into pGEX4T-1 vector. A TEV cleavage site was engineered between GST and Puc ${ }^{1-390}$. The expression and purification of Puc ${ }^{1-390}$ was performed in the way same as the purification of SPOP $^{\mathrm{BTB}+}$ described earlier in this chapter. Individually purified SPOP ${ }^{\mathrm{MATH}}$ and $\mathrm{Puc}^{1-390}$ were mixed at a molar ratio of $1: 1$ and further purified by gel filtration chromatography using an SD200 column. The SPOP ${ }^{\mathrm{MATH}}-\mathrm{Puc}^{1-390}$ protein complex was diluted with PBS to around 1 $\mathrm{mg} / \mathrm{ml}$. Trypsin was diluted to a series of concentrations of $1,0.3,0.1,0.03,0.01$, and 
$0.003 \mathrm{mg} / \mathrm{ml} .1 \mu \mathrm{l}$ of each diluted trypsin concentration was added to different tubes containing $9 \mu 1 \mathrm{SPOP}^{\mathrm{MATH}}-\mathrm{Puc}^{1-390}$. The individual trypsin/ $\mathrm{SPOP}^{\mathrm{MATH}}-\mathrm{Puc}^{1-390}$ mixtures were incubated on ice for two hours. Stable bands representing protein fragments resistant to trypsin digestion were separated and observed on a coomassie blue stained SDS-PAGE gel for the samples with $1 \mu l 0.03 \mathrm{mg} / \mathrm{ml}$ trypsin added to $9 \mu 11 \mathrm{mg} / \mathrm{ml}$ $\mathrm{SPOP}^{\mathrm{MATH}}-\mathrm{Puc}^{1-390}$. To identify the trypsin-resistant fragments, this digestion ratio was further used for larger scale preparation. $1 \mu 1 \mathrm{of} 1 \mathrm{mg} / \mathrm{ml}$ trypsin was added to $300 \mu \mathrm{l}$ of $1 \mathrm{mg} / \mathrm{ml} \mathrm{SPOP}^{\mathrm{MATH}}-\mathrm{Puc}^{1-390}$ and the protein mixture was incubated on ice for two hours, followed by gel filtration chromatography using an SD200 column to separate digestion products based on size. Protein fragments in each eluate fraction were characterized by mass spectrometry, performed by Dr. David King (Howard Hughes Medical Institute). Furthermore, $20 \mu \mathrm{l}$ of each fraction were loaded on $15 \%$ SDS-PAGE and stained with coomassie blue to obtain an approximate view of the separated digest products.

\subsubsection{Identification of a MacroH2A Sequence Required for Binding to SPOP}

GST pull down experiments were performed to map the SPOP binding region on MacroH2A by using a series of deletion mutants. The idea behind these experiments was that SPOP ${ }^{\mathrm{MATH}}$ would copurify with GST-tagged fragments of MacroH2A containing a SPOP-binding sequence, but would not copurify with either GST (control) or GST-tagged fragments of MacroH2A in which the SPOP-binding site had been deleted. MacroH2A ${ }^{166-372}$ and MacroH2A ${ }^{180-372}$ were cloned into pGEX4T-1. SPOP ${ }^{\mathrm{MATH}}$ was cloned into pRSFDuet with His-MBP fusion at the $\mathrm{N}$ terminus. The vector expressing SPOP $^{\mathrm{MATH}}$ was co-transformed with one of the vectors expressing GST, or a GST-MacroH2A deletion mutant, into BL21 (DE3) GOLD cells. Cultures were grown in LB media containing a final concentration of $50 \mu \mathrm{g} / \mathrm{ml}$ kanamycin and $200 \mu \mathrm{g} / \mathrm{ml}$ ampicillin. Cell lysate from $1 \mathrm{~L}$ culture was incubated with $1 \mathrm{ml}$ glutathione sepharose $4 \mathrm{~B}$ resin at $4{ }^{\circ} \mathrm{C}$ for 1 hour, with gentle rocking. Then the sepharose beads were loaded onto a small gravity column, and washed extensively with Tris buffer. Proteins were eluted with $10 \mathrm{mM}$ glutathione and separated on $15 \%$ SDS PAGE, stained with coomassie blue.

\subsection{PROTEIN INTERACTION STUDIES}

\subsubsection{Interactions between SPOP ${ }^{\mathrm{MATH}}$ and SBC Sequences}

To study the interactions between $\mathrm{SPOP}^{\mathrm{MATH}}$ and different $\mathrm{SBC}$ peptides, surface plasmon resonance (SPR) was used. SPR is a technique that allows the real-time detection and monitoring of bio-molecular bindings. For example, to study the interactions between molecule $\mathrm{A}$ and molecule $\mathrm{B}$, molecule $\mathrm{A}$ is bound to the biosensor surface (sensor chip) and molecule B is delivered to the surface in a continuous flow. If B binds immobilized A, the binding will cause a change in mass at the surface, which can be detected as surface plasmon resonance. Changes in surface plasmon resonance are 
measured in real time. The response is directly related to the mass of molecules that bind to the surface, therefore, the binding kinetics, stoichiometries and affinity constants can be calculated from this experiment. Usually, immobilized molecule A is referred to as the ligand and molecule B is referred to as the analyte. The experimental setup of SPR requires relatively less ligand than analyte. Thus, our experiments were designed such that the reagent with more restricted availability was the immobilized ligand. Given the limited production of SPOP ${ }^{\text {MATH }}$ protein and high yield of peptide synthesis, SPOP ${ }^{\text {MATH }}$ was used as ligand and the potential SBC peptides were used as analytes.

Another issue in designing SPR experiments is the choice of chip. There are several types of sensor chips that can be used to non-specifically bind proteins as ligands. However, we considered that non-specific binding would have the potential to affix the substrate-binding site of the SPOP MATH domain to the BIACORE chip, which would preclude binding to SBC peptides. Thus, we chose a method for specifically affixing the ligand to the chip. We fused GST at the $\mathrm{N}$ terminus of SPOP ${ }^{\mathrm{MATH}}$ and captured GST-SPOP $^{\mathrm{MATH}}$ on a GST antibody coated sensor chip. The GST-SPOP ${ }^{\mathrm{MATH}}$ wild type and mutants were purified as described in the structure determination sections of this chapter. The SPR experiments were performed by Brett Waddell at the St. Jude Hartwell Center. The procedures are briefly described below.

Binding studies were performed at $25^{\circ} \mathrm{C}$ using a BIACORE 3000 (GE Healthcare) SPR instrument. Anti-GST antibodies (GE Healthcare) were covalently attached to a carboxymethyl dextran-coated gold surface (CM-4 Chip; GE Healthcare). The carboxymethyl groups of dextran were activated with NHS (N-hydroxysuccinimide) and EDC (N-ethyl-N' -(3-dimethylaminopropyl) carbodiimide and), and anti-GST antibodies were attached at $\mathrm{pH} 5.0$ in $10 \mathrm{mM}$ sodium acetate. Any remaining reactive sites were blocked by reaction with ethanolamine. The kinetics of association and dissociation were monitored at a flow rate of $75 \mu \mathrm{l} / \mathrm{min}$. GST-tagged ligands were captured to a level of $\sim 500-1000 \mathrm{RU}$ (resonance unit) for each experiment. GST was captured on the reference surface to account for any non-specific binding to the GST tag. The peptide analytes were prepared in $20 \mathrm{mM}$ Tris (pH 7.6), $150 \mathrm{mM} \mathrm{NaCl}, 0.1 \mathrm{mg} / \mathrm{ml}$ BSA, and $0.005 \%$ P20 surfactant. Peptide concentrations used for binding analysis were variable and are reported in the attached results table. To account for injection artifacts, a series of sensorgrams were recorded throughout the experiment after injecting only buffer (blank injections). The analytes dissociated completely from the chip surfaces, eliminating the need for a regeneration step. Data reported are the difference in SPR signal between the flow cell containing the GST-tagged ligand and the reference cell with GST only. Additional instrumental contributions to the signal were removed by subtraction of the average signal of the blank injections from the reference-subtracted signal. Triplicate injections were made, and the data were analyzed by equilibrium affinity analysis using the software package Scrubber 2 (Version 2.0b, BioLogic Software).

In order to examine protein interactions in a cellular context, we turned to a cell culture protein expression system. These experiments were performed by Dr. Jiang Liu in Dr. Kevin White's lab at the University of Chicago. Drs. Liu and White recently 
reported the interaction of the Drosophila ortholog of SPOP (HIB) with the phosphatase, Puc [58]. Therefore, experiments were performed using Drosophila cell culture/expression systems. Briefly, Drosophila S2 cells were cultured in Schneider's Drosophila Medium with $10 \% \mathrm{FBS}$ at $25^{\circ} \mathrm{C}$. DNA transfections were performed using Effectene Transfection Reagent (QIAGEN), according to the manufacturer's instructions. Two days after transfection of HIB and either Puc, or structure-based mutant versions of the proteins, the cells were lysed with RIPA buffer. Cell lysates were centrifuged at $10,000 \mathrm{~g}$ for $10 \mathrm{~min}$ at $4{ }^{\circ} \mathrm{C}$. A common method for examing protein-protein interactions in cells is to perform co-immunoprecipitation, whereby one protein in a complex is recognized by an antibody, and the other is not recognized by the antibody but copurifies with its partner proteins. For these experiments, HIB was expressed with an N-terminal flu Hemagglutinin (HA) tag and Puc was expressed with an N-terminal Myc tag. Thus, HIB would only associate with anti-Myc beads in the presence of Puc. Futhermore, mutations that prevent coassociation would also prevent HA-HIB from copurifying with Myc-Puc over anti-Myc beads. Supernatants were incubated with anti-Myc conjugated agarose beads (Sigma, rabbit antibody) for 4 hours at $4{ }^{\circ} \mathrm{C}$. The beads were washed and the bound protein complex was eluted in SDS-Laemmli buffer. The HIB and Puc proteins were detected by immunoblotting with anti-HA and anti-Myc antibodies, respectively.

\subsubsection{Interactions between $\mathrm{SPOP}^{\mathrm{BTB}+}$ and $\mathrm{Cul3}$}

$\mathrm{SPOP}^{\mathrm{BTB}+}$ was purified as described in the structural biology section of this chapter. We were able to make a Cul3 $\mathrm{N}$ terminal fragment $\mathrm{Cul} 3^{\mathrm{NTD}}$ (residues 1-384) as follows. Cul3 ${ }^{\text {NTD }}$ was cloned into the pABLO GST-fusion coexpression vector (gift of Alona Cohen and Nikola Pavletich). GST-Cul3 ${ }^{\text {NTD }}$ was purified the way same as other GST tagged protein purifications as described in earlier this chapter. TEV was added at a mass ratio of 1:100 and the protein was dialyzed against $50 \mathrm{mM}$ Tris $\mathrm{pH} 7.6,150 \mathrm{mM}$ $\mathrm{NaCl}$ and $5 \mathrm{mM}$ DTT. The GST and $\mathrm{Cul}^{\mathrm{NTD}}$ mixture was passed back on a glutathione sepharose column to get rid of GST. The dialysis step is essential to remove the reduced glutathione from the initial affinity purification, so that the free GST and uncleaved $\mathrm{GST}_{-\mathrm{Cul}}{ }^{\mathrm{NTD}}$ is removed during the pass-back.

To study the interactions between $\mathrm{SPOP}^{\mathrm{BTB}+}$ and $\mathrm{Cul}^{\mathrm{NTD}}$, firstly, a nondenaturing gel protein mobility shift assay was performed. Briefly, unlike in an SDS gel where proteins are denatured by binding to the SDS detergent and thus cannot associate via noncovalent interactions, in a nondenaturing acrylamide gel there are no denaturants. Thus, proteins are in their native form and can co-associate via noncovalent interactions. Binding reactions were performed with $50 \mu \mathrm{M} \mathrm{SPOP}^{\mathrm{BTB}}{ }^{+}, 20 \mu \mathrm{M} \mathrm{Cul} 3^{\mathrm{NTD}}$ in $5 \mu 1$ volumes in $20 \mathrm{mM}$ Tris, $150 \mathrm{mM} \mathrm{NaCl}, 5 \mathrm{mM}$ DTT, pH 7.6, for one hour. Free $\mathrm{SPOP}^{\mathrm{BTB}+}$ and $\mathrm{Cul}^{\mathrm{NTD}}$ were separated from $\mathrm{SPOP}^{\mathrm{BTB}+}-\mathrm{Cul}^{\mathrm{NTD}}$ complexes on a $4.5 \%$ polyacrylamide gel in a nondenaturing buffer of $90 \mathrm{mM}$ Tris borate, $2 \%$ glycerol, $\mathrm{pH}$ 8.0, and were visualized with Coomassie staining. 
As a second method to study the interactions between $\mathrm{SPOP}^{\mathrm{BTB}+}$ and $\mathrm{Cul} 3^{\mathrm{NTD}}$, we performed analytical gel filtration chromatography. Gel filtration chromatography is a method of separating proteins in their native form based on size. Since protein complexes are larger than their individual components, gel filtration is a method for identifying propensities for proteins to form a complex. Large proteins or complexes come off a gel filtration column earlier than small proteins or complexes. Gel filtration chromatography was performed on isolated $\mathrm{SPOP}^{\mathrm{BTB}+}$ and $\mathrm{Cul}^{\mathrm{NTD}}$ individually using a $24 \mathrm{ml}$ Superdex 200 column at $4{ }^{\circ} \mathrm{C}$ in buffer $20 \mathrm{mM}$ Tris (pH 7.6), $150 \mathrm{mM} \mathrm{NaCl}$ and $5 \mathrm{mM}$ DTT. The same experiment was also performed with the $\mathrm{SPOP}^{\mathrm{BTB}+}-\mathrm{Cul}^{\mathrm{NTD}}$ mixture after two proteins were mixed at 1:1 molar ratio and incubated on ice for one hour. The earlier elution of the $\mathrm{SPOP}^{\mathrm{BTB}+}-\mathrm{Cul}^{\mathrm{NTD}}$ mixture reflected formation of a $\mathrm{SPOP}^{\mathrm{BTB}}-\mathrm{Cul}^{\mathrm{NTD}}$ complex.

A limitation of both the native gel shift and analytical gel filtration assays is that they are not quantitative. To obtain quantitative information in $\mathrm{SPOP}^{\mathrm{BTB}+}$ and $\mathrm{Cul} 3^{\mathrm{NTD}}$ binding, Dr. Amanda Nourse at the St. Jude Hartwell Center performed analytical ultracentrifugation (AUC), using proteins we purified. In an AUC experiment, samples are spun at high speed and molecules with different weight accumulate at different positions in the spinning cell with different spinning radiuses. The distribution of the molecules can be monitored in real time through the optical detection system and the molecule features (such as molecular weight and gross shape) can be calculated based on the sample concentration versus the axis of rotation profile under the applied centrifugal field. Two types of AUC experiments are commonly used: sedimentation velocity experiments and sedimentation equilibrium experiments. The gross shape, the conformational changes, and the size distributions of proteins can be obtained from sedimentation velocity experiments; the subunit stoichiometry of protein complexes and equilibrium constants can be obtained from sedimentation equilibrium experiments. In this study, both experiments were used. $\mathrm{SPOP}^{\mathrm{BTB}}$ was purified the same way as $\mathrm{SPOP}^{\mathrm{BTB}+}$. $\mathrm{SPOP}^{\mathrm{BTB}+}-\mathrm{Cul}^{\mathrm{NTD}}$ and $\mathrm{SPOP}^{\mathrm{BTB}}-\mathrm{Cu}^{\mathrm{NTD}}{ }^{\mathrm{NTD}}$ complexes were formed and purified by mixing purified components together by gel filtration chromatography.

Protein samples of $\mathrm{SPOP}^{\mathrm{BTB}+}, \mathrm{SPOP}^{\mathrm{BTB}}, \mathrm{SPOP}^{\mathrm{BTB}+}-\mathrm{Cul}^{\mathrm{NTD}}$ complex and $\mathrm{SPOP}^{\mathrm{BTB}}$-Cul3 $^{\mathrm{NTD}}$ complex in $25 \mathrm{mM}$ Tris $\mathrm{pH} 7.5,150 \mathrm{mM} \mathrm{NaCl}$ and $5 \mathrm{mM}$ DTT were subjected to sedimentation velocity ultracentrifugation in a ProteomeLab XL-I analytical ultracentrifuge with a Beckman An-60 Ti rotor and cells containing sapphire windows and charcoal-filled Epon double-sector centerpieces. The density and viscosity of the buffer were calculated from their composition, and the partial specific volume and molecular weights of the protein and complexes were calculated based on their amino acid composition using the software SEDNTERP. Ultracentrifugation was performed at $60,000 \mathrm{rpm}$ for $12 \mathrm{hr}$ at $4{ }^{\circ} \mathrm{C}$. Sedimentation equilibrium experiments were performed with a Beckman An-50 Ti rotor and cells containing quartz windows and charcoal-filled Epon double-sector centerpieces. Equilibrium was attained at $48 \mathrm{~h}$ at a rotor temperature of $4{ }^{\circ} \mathrm{C}$ at increasing speeds of 8,12 and $15 \mathrm{k} \mathrm{rpm}$. Protein at concentrations of between 1.23-8.0 $\mu \mathrm{M}(180 \mu \mathrm{L})$ was loaded into double-sector centrepieces and absorbance distributions recorded at 280 in $0.001 \mathrm{~cm}$ radial intervals with 20 replicates for each 
point. Global least squares modelling were performed at multiple rotor speeds with the software SEDPHAT.

\subsection{UBIQUITINATION ASSAY}

\subsubsection{In Vitro Ubiquitination Assay}

In order to examine the relevance of the structurally-observed SPOP interactions for SPOP function as part of an E3 ubiquitin ligase, it is necessary to test the effects of mutations using a ubiquitination assay. Transfer of ubiquitin requires an E1-E2-E3 enzyme cascade, which functions in an ATP-dependent manner (E1 utilizes ATP during ubiquitin activation). We reconstituted this cascade in vitro with purified components. The ubiquitination assay was set up at room temperature with purified protein components in 50mM Tris $\mathrm{pH} 7.6,150 \mathrm{mM} \mathrm{NaCl}, 10 \mathrm{mM} \mathrm{MgCl}_{2}, 5 \mathrm{mM}$ ATP and $1 \mathrm{mM}$ DTT. Human ubiquitin E1 and E2 (UbcH5b) were purified as previously described [24]. The Cul3 component of the E3 was purified using the split/co-expression method and modified with NEDD8 [69]. The reaction contained $250 \mathrm{nM} \mathrm{E1,} 2 \mu \mathrm{M} \mathrm{UbcH5b}, 2 \mu \mathrm{M}$ Cul3-N8, $2 \mu \mathrm{M}$ SPOP (component of E3), $5 \mu \mathrm{M}$ His-Puc (substrate), $50 \mu \mathrm{M}$ ubiquitin and $2 \mathrm{mg} / \mathrm{ml}$ bovine serum albumin (BSA; carrier protein necessary for optimal ubiquitin activation by E1). 2X SDS loading dye was added to stop the reaction at $15 \mathrm{~min}, 45 \mathrm{~min}$ and 90 min. Reaction mixtures were resolved on 4-12\% Bis-Tris SDS-PAGE and His-Puc was detected by western blotting using anti-PentaHis antibody (QIAGEN).

\subsubsection{In Vivo Ubiquitination Assay}

In vivo ubiquitination assays were performed by Dr. Jiang Liu at the University of Chicago, based on the protocol previously described [58]. As with the coimmunoprecipitation binding assays described above, Drosophila SPOP and Puc were studied. Briefly, Drosophila S2 cells were transfected in 6-well plates with $1 \mu \mathrm{g}$ UAS-Myc-Puc, and $0.6 \mu \mathrm{g}$ each of the following constructs: UAS-HA-D-SPOP or pMT-HA-Ub, and Actin-Gal4. $24 \mathrm{hr}$ after transfection, $700 \mu \mathrm{M} \mathrm{CuSO}_{4}$ was added to the medium for another $24 \mathrm{hr}$ to induce HA-Ub expression. Cells were treated with $50 \mu \mathrm{M}$ of the proteasome inhibitor MG132 for $4 \mathrm{hr}$ before harvesting. It was necessary to add proteasome inhibitor to these experiments to prevent the reaction product (ubiquitinated Puc) from being degraded. Cells were first lysed in denaturing buffer (1\% SDS, $50 \mathrm{mM}$ Tris $\mathrm{pH} 7.5,0.5 \mathrm{mM}$ EDTA, $1 \mathrm{mM}$ DTT). After incubation for $5 \mathrm{~min}$ at $100^{\circ} \mathrm{C}$, the lysates were diluted 10 times with lysis buffer and then subjected to immunoprecipitation with Anti-c-Myc conjugated agarose beads (Sigma, rabbit antibody), followed by immunoblot analysis with a myc antibody (Invitrogen). 


\subsection{SMALL ANGLE X-RAY SCATTERING ANALYSIS}

Small angle X-ray scattering analysis (SAXS) was performed by Dr. Michal Hammel at Lawrence Berkley National Laboratory to study the conformation of SPOP in solution. SAXS is a technique that measures the elastic scattering of X-rays by samples in solution. The diffraction pattern contains information about the shape and size of macromolecules and characteristic distances of partially ordered materials. The advantage of SAXS over crystallography is that it does not require protein to form crystals and it studies protein in solution, which is more likely to adopt native folding. The disadvantage is that it is a low-resolution technique resolving structures in the nanometer range. Since we have obtained the high-resolution structure of $\mathrm{SPOP}^{\mathrm{MATH}-\mathrm{BTB}+}$ and we wanted to study the conformational arrangements between the MATH and BTB domain in solution, SAXS was the ideal technique to use.

Dr. Hammel performed SAXS analysis with two protein samples we purified, $\mathrm{SPOP}^{\mathrm{MATH}-\mathrm{BTB}+}$ and SPOP ${ }^{\mathrm{N}-\mathrm{MATH}-\mathrm{BTB}^{+}}$. Both proteins were prepared as described above for crystallization. The solution scattering data were collected at the ALS beamline 12.3.1 LBNL Berkeley, California and processed as previously described [137]. Tunable wavelength $(\lambda)$ and the sample-to-detector distances were set to $1.0-1.5 \AA$ and $1.5 \mathrm{~m}$, respectively, resulting in scattering vectors (q) ranging from $0.008 \AA^{-1}$ to $0.31 \AA^{-1}$ for $\mathrm{q}=$ $4 \pi \cdot \sin (\theta) / \lambda$, where $2 \theta$ is the scattering angle. SAXS data at short and long time exposures $(0.5 \mathrm{~s}, 5 \mathrm{~s})$ were merged to define the entire scattering profile. Different protein concentrations were tested for aggregation and examined by Guinier plots [138]. The radius of gyration $\left(\mathrm{R}_{\mathrm{G}}\right)$ was derived by the Guinier approximation $I(q)=I(0) \exp (-$ $\left.q^{2} R_{G}{ }^{2} / 3\right)$ with the limits $q \mathrm{R}_{\mathrm{G}}<1.3$. The curves measured for different proteins concentrations $(1.6-6.0 \mathrm{mg} / \mathrm{ml})$ displayed no concentration dependence. Scattering curves were used to calculate the pair-distance distribution functions $(\mathrm{P}(\mathrm{r}))$ and define the maximum dimension of the macromolecules $\left(\mathrm{D}_{\max }\right)$ using GNOM [139]. In the rigid body modeling strategy BILBOMD, molecular dynamics (MD) simulations are used to explore conformational space [140]. A common strategy is to perform the MD simulation on the domains connections at very high temperature, where the additional kinetic energy prevents the molecule from becoming trapped in a local minimum. The MD simulations provide an ensemble of molecular models from which a SAXS curve is calculated and compared to the experimental curve. A genetic algorithm is used to identify the EOM (Ensemble Optimization Method) required to best fit the experimental data [140]. The requirement for the EOM approach is an indicator that the SPOP structures are flexible in solution. 


\section{CHAPTER 3. SUBSTRATE RECOGNITION BY SPOP MATH DOMAIN}

\subsection{INTRODUCTION}

$\mathrm{SPOP} / \mathrm{HIB}$ is a BTB protein that serves as the substrate adaptor for Cul3-based ubiquitin ligase to mediate ubiquitination of divergent substrates in various signaling pathways. In Drosophila, the Hedgehog (Hh) signaling pathway governs cell growth and plays critical roles in animal development. The degradation of the key transcriptional effector $\mathrm{Ci}$ protein is highly regulated by an ubiquitin ligase, which consists of $\mathrm{Cul} 3$ and HIB (Hh induced MATH and BTB protein) [4, 125, 141]. In addition, HIB negatively regulates Tumor Necrosis Factor signaling in the Drosophila eye by promoting turnover of the MAPK phosphatase Puckered (Puc) [58]. SPOP (speckle-type POZ protein), the human ortholog of HIB, was first identified as a MacroH2A associate during chromatin remodeling [123]. In human cells, the $\mathrm{Cul} 3^{\mathrm{SPOP}}$ ligase promotes monoubiquitination of MacroH2A during stable X chromosome inactivation [122]. $\mathrm{Cul3}^{\text {SPOP }}$ also targets Daxx for ubiquitination, thereby controlling Daxx-dependent transcriptional repression of pro-apoptotic proteins such as p53 [116].

The mechanism of substrate recognition by SPOP/HIB is only partly understood. The N terminus MATH (meprin-associated TRAF homology) domain of SPOP was shown to specifically interact with both the $\mathrm{N}$-terminal and $\mathrm{C}$-terminal sequences of $\mathrm{Ci}$ and non-histone domain of MacroH2A [4, 123]. However, no obvious domains or consensus sequences of substrates have been identified.

SPOP/HIB MATH domain is a subtype of TRAF (tumor necrosis factor-receptor associated factor) domain in TRAF family proteins (TRAF1, TRAF2, TRAF3, TRAF4, TRAF5, and TRAF6) involved in TNF family receptor signaling. The MATH and TRAF domains are predicted to adopt similar overall structures. SPOP MATH cannot inhibit NF-kB induction while classical TRAF domains can, indicating distinct biological functions of SPOP MATH and classical TRAF [119, 142]. Recently, MATH domain has also been identified from other proteins, such as MUL, the product of the causative gene in Mulibrey Nanism syndrome [142, 143] and HAUSP (herpesvirus-associated ubiquitin specific protease), an ubiquitin protease $[142,144]$. Notably, HAUSP MATH domain is also capable of binding various substrates [16, 144-146].

To gain a deeper understanding of substrate recognition by $\mathrm{SPOP} / \mathrm{HIB}^{\mathrm{MATH}}$, we set out to determine the mechanisms, binding affinities, and structure basis of $\mathrm{SPOP}^{\mathrm{MATH}}$-substrate interactions. In this chapter, we report the identification of SPOP binding consensus (SBC) in four SPOP substrates, including Puc, MacroH2A, Ci and Daxx, and four crystal structures of SPOP ${ }^{\mathrm{MATH}}$ in complex with different substrate peptides. The structures reveal the molecular basis for the binding of substrate to $\mathrm{Cul3}^{\mathrm{SPOP}}$ ubiquitin ligase. In addition, these findings have broad implications for understanding the role of SPOP in the Hedgehog, TNF, X chromosome inactivation and cell death pathways. 


\subsection{RESULTS}

\subsubsection{Identification of SPOP Binding Consensus (SBC) in Multiple SPOP Substrates}

SPOP binds substrate through the N-terminal MATH domain (residue 28-166). In order to understand SPOP ${ }^{\mathrm{MATH}}$-substrate interactions, we identified SPOP ${ }^{\mathrm{MATH}}$ interacting regions on the substrates Puc and MacroH2A. Puc is a Drosophila protein and MacroH2a is a human protein. Since SPOP ${ }^{\mathrm{MATH}}$ and $\mathrm{HIB}^{\mathrm{MATH}}$ sequences are $94 \%$ identical, SPOP ${ }^{\mathrm{MATH}}$ was used to study the binding to both Puc and MacroH2A.

\subsubsection{Identification of a SPOP-binding peptide in Puc}

The full length Puc, containing 476 amino acids, can be divided into three domains: NTD ${ }^{\mathrm{N}-132}$, phosphotase domain (133-267) and $\mathrm{CTD}^{268-\mathrm{C}}$. We first found that a $42 \mathrm{kD}$ fragment of Puc, residues 1-390, formed a stable complex with full-length SPOP as well as SPOP ${ }^{\mathrm{MATH}}$ (Appendix A, Fig A.2). Then we mapped the minimal SPOP binding element by limited proteolysis (Fig 3.1). After trypsin treatment of purified SPOP $^{\mathrm{MATH}}{ }_{-} \mathrm{Puc}^{1-390}$ protein complex, a small Puc peptide encompassing amino acids 91-113, that comigrated with SPOP ${ }^{\mathrm{MATH}}$ by gel filtration chromatography, was identified by mass spectrometry, suggesting residues 91-ENLACDEVTSTTSSSTAMNGGGR-113 within Puc $\mathrm{NTD}^{\mathrm{N}-132}$ were sufficient for SPOP ${ }^{\mathrm{MATH}}$ binding.

\subsubsection{Identification of a MacroH2A sequence required for binding to SPOP}

MacroH2A has two domains: Histone-like domain and non-Histone-like domain. Previous studies have shown that the Histone-like domain of MacroH2A was not responsible for SPOP interaction [123]. We further narrowed SPOP binding site on MacroH2A to 14 amino acids by GST pull down experiments (Fig 3.2). MacroH2A residues 166-KAASADSTTEGTPAD-179, which reside between Histone-like and non-Histone-like domains, were necessary for SPOP ${ }^{\mathrm{MATH}}$ binding. This result was further strengthened by the ability of a synthetic peptide corresponding to MacroH2 $\mathrm{A}^{166-179}$ to bind SPOP ${ }^{\mathrm{MATH}}$ (data in section 3.2.1.3).

\subsubsection{Identification of SPOP-binding sites in substrates $\mathrm{Ci}$ and Daxx}

The finding of two short peptides from different substrates interacting with SPOP $^{\mathrm{MATH}}$ raised the question: Do Puc and MacroH2A have common SPOP binding sites? To address this question, we examined the sequences of $\mathrm{Puc}^{93-107}$ and MacroH2A ${ }^{166-179}$. Notably, a common sequence, three amino acids STT, was found in both peptides (Fig 3.1 and Fig 3.2), serving as a potential motif. However, we were not able to find the same sequence within $\mathrm{Ci}$ and Daxx, the other two SPOP substrates. Since Serine and Threonine have similar side chain properties, we extended the motif search to three consecutive Ser/Thr combinations, S/T-S/T-S/T instead of STT. Multiples sites were identified in different substrates (Fig 3.3). In addition to S/T-S/T-S/T, we noticed two more amino acids preceding $\mathrm{S} / \mathrm{T}-\mathrm{S} / \mathrm{T}-\mathrm{S} / \mathrm{T}$ were consistent in polarity while the rest of 


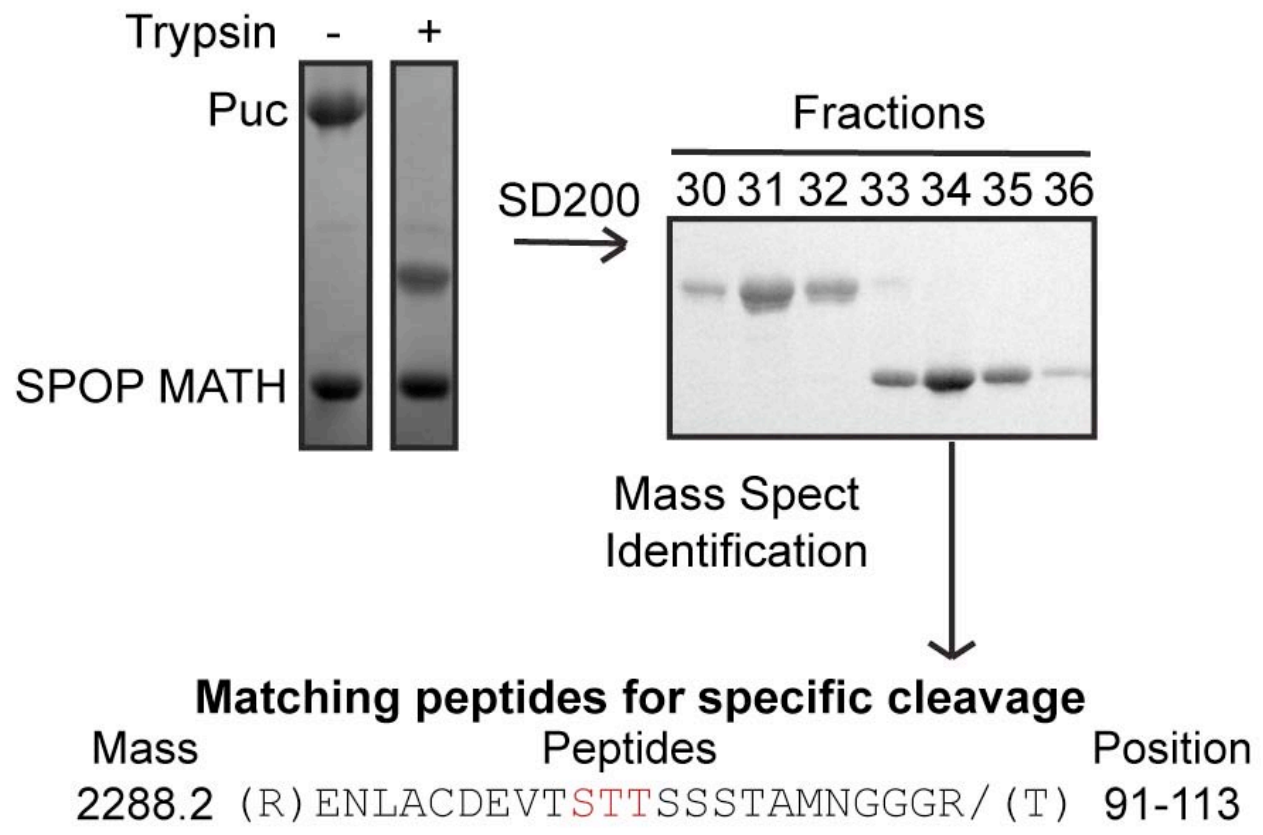

Figure 3.1. Identification of a SPOP-binding peptide in Puc. Left, Coommassiestained SDS-PAGE gel showing products after trypsin digestion of a purified SPOP $^{\mathrm{MATH}}{ }_{-P u c^{1-390}}$ complex (1:333 trypsin:SPOP ${ }^{\mathrm{MATH}}-\mathrm{Puc}^{1-390}, 3 \mathrm{hrs}$, room temperature). Right, Coommassie-stained SDS-PAGE gel of fractions from gel filtration (SD200) separation of trypsin digested products. Bottom, peptide co-purifying with SPOP ${ }^{\mathrm{MATH}}$ in fractions 33-35, identified by mass spectrometry. 

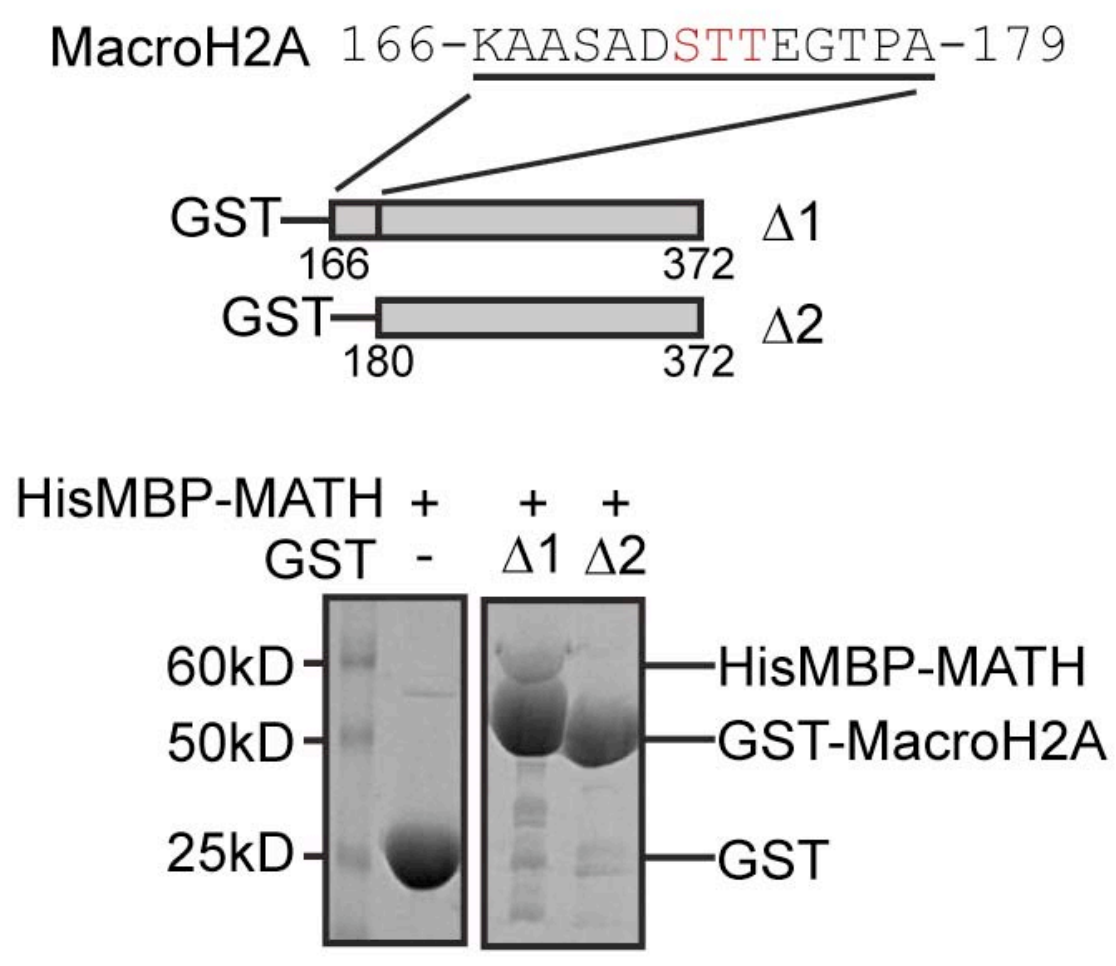

Figure 3.2. Identification of a MacroH2A sequence required for binding to SPOP. Top, Schematic view of MacroH2A deletion constructs, highlighting sequence of residues 166-179. Bottom, Coommassie-stained SDS-PAGE gel of GST-pull-downs of GST, GST-MacroH2A $\Delta 1$, and GST-MacroH2A $\Delta 2$ coexpressed with HisMBP-SPOP ${ }^{\mathrm{MATH}}$ in E. coli. 


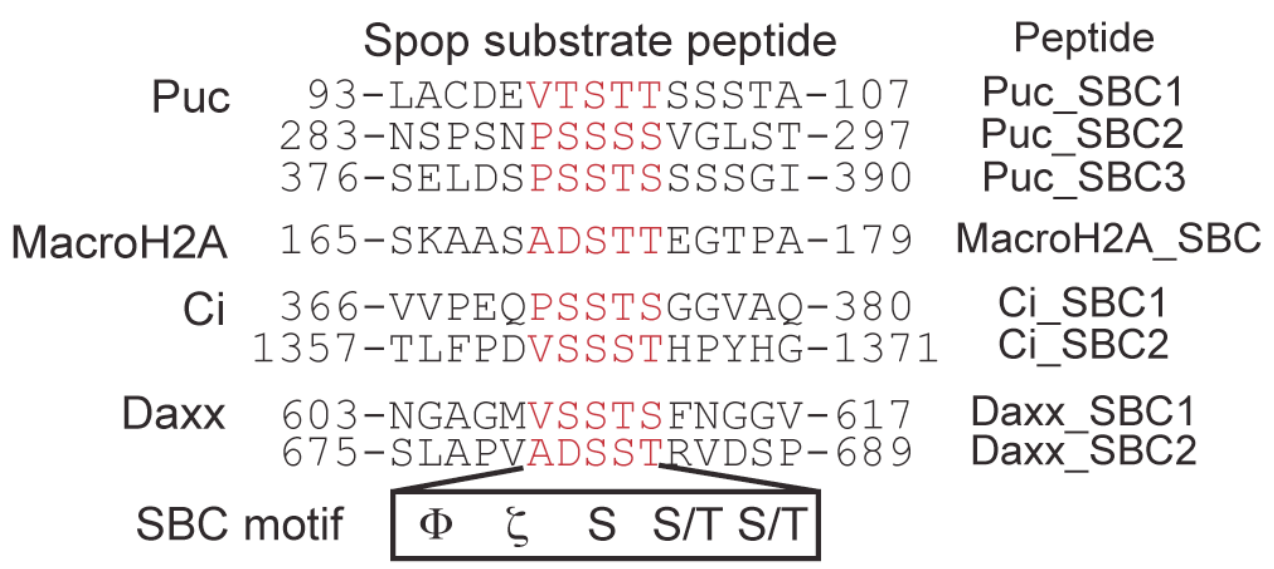

Figure 3.3. Identification of consensus sequences within four SPOP substrates. The consensus sequences are shown in red, in the middle of peptides corresponding to sequences of SPOP substrates Puc, MacroH2A, Ci and Daxx. 
peptides did not show obvious sequence similarity. Thus, a common sequence can be presented as $\phi-\varsigma-\mathrm{S} / \mathrm{T}-\mathrm{S}-\mathrm{S} / \mathrm{T}$, where $\phi$ denotes a non-polar residue and $\varsigma$ represents a polar residue.

This sequence serves as a SPOP binding consensus (SBC) motif, where synthetic peptides harboring this motif bind $\mathrm{SPOP}^{\mathrm{MATH}}$ with a range of affinities, as detected using Surface Plasmon Resonance (BIACORE) (Fig 3.4). Notably, Puc, Ci, and Daxx all harbor multiple SBCs, as do several other Cull-based CRL substrates. This is also in agreement with the paradigm established by Keap1 substrate binding Kelch domain, which binds two distinct sites on the substrate Nrf2. The Puc SBC1 that co-purified with $\mathrm{SPOP}^{\mathrm{MATH}}$ by gel filtration displayed the highest affinity at $13 \mu \mathrm{M}$.

3.2.1.4 SBC motif mediated SPOP-substrate interaction is required for ubiquitination in vitro

We sought to determine whether the interactions between SPOP ${ }^{\mathrm{MATH}}$ and the Puc SBCs play roles in in vitro ubiquitination. As with other CRLs, SPOP-Cul3 mediated ubiquitination of Puc in the presence of E1 and E2 (Appendix A, Fig A.3). To investigate the involvement of individual Puc SBCs in ubiquitination, we tested the effects of their mutation on in vitro ubiquitination of Puc. The results indicate that all three SBCs contribute to Puc ubiquitination and their contributions are proportional to their ability to bind SPOP (Fig 3.5).

\subsubsection{Structural Basis of SPOP ${ }^{\mathrm{MATH}}-\mathrm{SBC}$ Interactions}

The SPOP MATH domain specifically recognizes the SBC motif, but not other random sequences. To understand the molecular basis for SPOP ${ }^{\mathrm{MATH}}-\mathrm{SBC}$ motif interactions, we determined four structures of $\mathrm{SPOP}^{\mathrm{MATH}}$ in complexes with substrate peptide from three substrates: SPOP ${ }^{\mathrm{MATH}}-\mathrm{MacroH} 2 \mathrm{~A}^{\mathrm{SBC}}$ pep1, SPOP ${ }^{\mathrm{MATH}}-\mathrm{MacroH} 2 \mathrm{~A}^{\mathrm{SBC}}$ pep2, SPOP ${ }^{\mathrm{MATH}}-\mathrm{Puc}^{\mathrm{SBC} 1}$ and $\mathrm{SPOP}^{\mathrm{MATH}}-\mathrm{Ci}^{\mathrm{SBC} 2}$.

\subsubsection{Overall structures of SPOP ${ }^{\mathrm{MATH}}-\mathrm{SBC}$}

SPOP $^{\mathrm{MATH}}$ comprises an anti-parallel beta sandwich, and the structures superimpose with other MATH domains, such as from TRAF2 [147, 148] and TRAF6 $[149,150]$, with r.m.s. deviation (RMSD) values ranging between $3.0 \AA$ to $3.4 \AA$ (Fig 3.6). In all the structures, the SBC peptides adopt extended conformations, and bind the MATH domain's central shallow groove (Fig 3.7).

Structures of SPOP ${ }^{\mathrm{MATH}}-\mathrm{MacroH} 2 \mathrm{~A}^{\mathrm{SBC}}$ (pep1) and SPOP ${ }^{\mathrm{MATH}}-\mathrm{MacroH} 2 \mathrm{~A}^{\mathrm{SBC}}$ (pep2) were determined. The sequences of peptides are "KAASADSTTEGTPAD" for MacroH2A ${ }^{\text {SBC }}$ (pep1) and "DSTTEGTPADGFTVL" for MacroH2A ${ }^{\text {SBC }}$ (pep2). SPOP $^{\mathrm{MATH}}$ binds the same residues on both peptides (Fig 3.8), demonstrating specific interactions between $\mathrm{SPOP}^{\mathrm{MATH}}$ and MacroH $2 \mathrm{~A}^{\mathrm{SBC}}$. The SPOP ${ }^{\mathrm{MATH}}-\mathrm{MacroH} 2 \mathrm{~A}^{\mathrm{SBC}}$ (pep1) structure was used for following interaction analysis. 


\begin{tabular}{|c|c|}
\hline \multicolumn{1}{|c|}{ Peptide } & $\mathbf{K}_{\mathrm{d}}(\boldsymbol{\mu} \mathbf{M})$ \\
\hline Puc_SBC1 & $13.1( \pm .1)$ \\
\hline Puc_SBC2 & $>500$ \\
\hline Puc_SBC3 & $300( \pm 1)$ \\
\hline MacroH2A_SBC & $79.9( \pm .3)$ \\
\hline Ci_SBC1 & $105( \pm 1)$ \\
\hline Ci_SBC2 & $161( \pm 2)$ \\
\hline Daxx_SBC1 & $>500$ \\
\hline Daxx_SBC2 & $227( \pm 2)$ \\
\hline Random pep1 & n/a \\
\hline Random pep2 & n/a \\
\hline Random pep3 & n/a \\
\hline
\end{tabular}
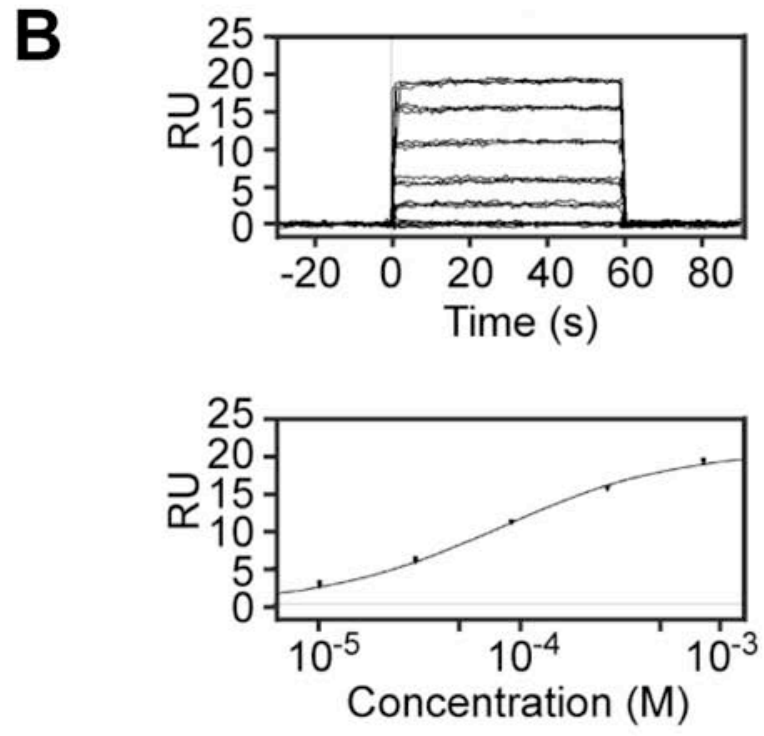

Figure 3.4. Interactions between SPOP ${ }^{\mathrm{MATH}}$ and SBC peptides. (A) Summary of binding constants for SPOP ${ }^{\mathrm{MATH}}$ interactions with SBC peptides, measured by Surface Plasmon Resonance (BIACORE3000). Random peptide sequences (pep1, LAPAATTSNEPS; pep2, SSAASGSYGH; and pep3, DPGEGPSTGPRG) were used as controls. (B) Representative BIACORE sensograms showing SPOP ${ }^{\mathrm{MATH}}$ binding to $\mathrm{MacroH} 2 \mathrm{~A}^{\mathrm{SBC}}$ : top, SPR response versus time; bottom, SPR response versus peptide concentration. 


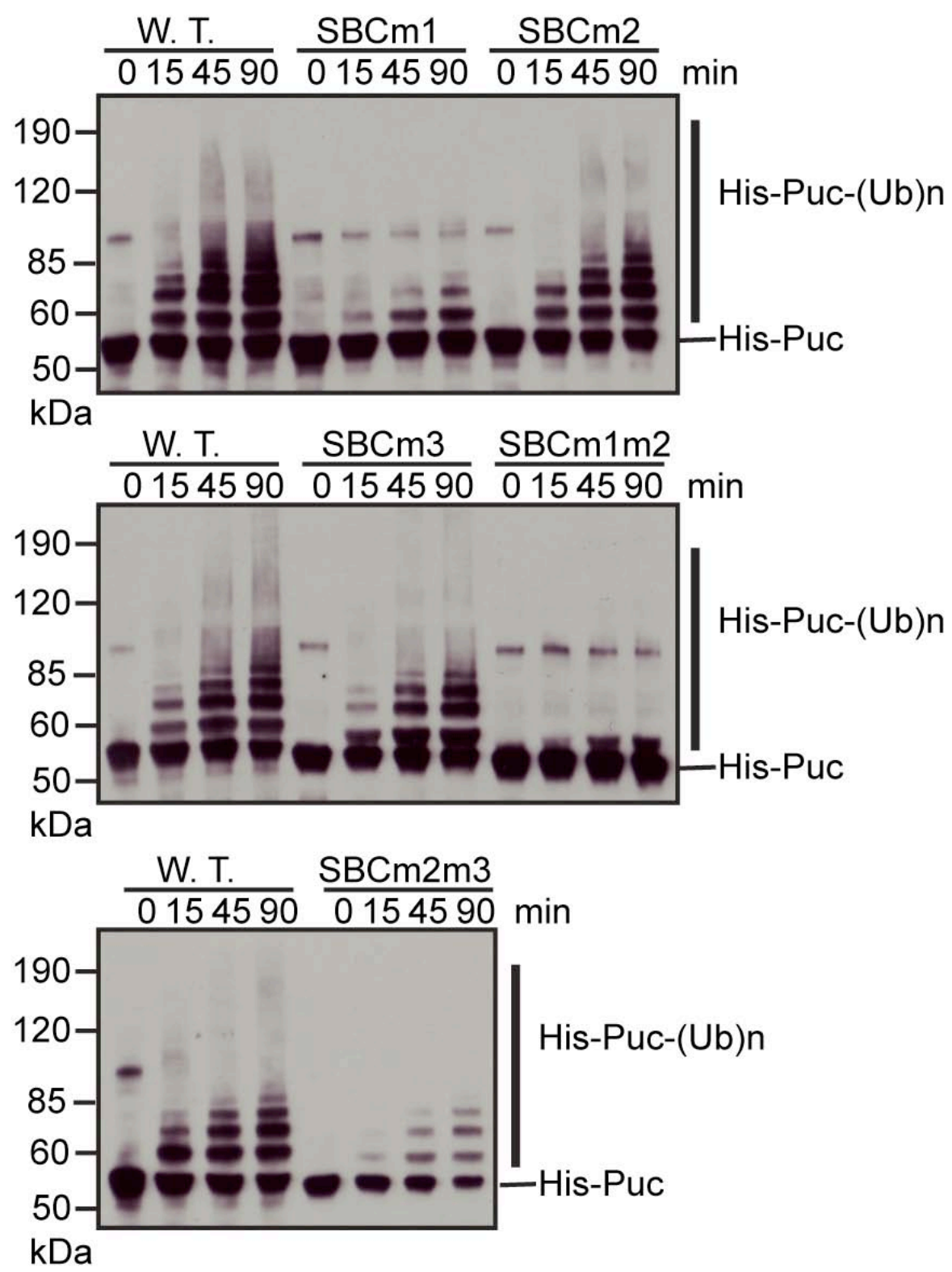

Figure 3.5. $\mathrm{SBC}$ is required for Puc ubiquitination in vitro. Western blots detecting His-Puc of in vitro ubiquitination assays for wild-type (W.T.) and mutant Puc substrates. In SBCm1, SBCm2 and SBCm3, the three SBC sites are individually substituted with the sequence GGSGS. SBCm1m2 and SBCm2m3 are double SBC mutants. 


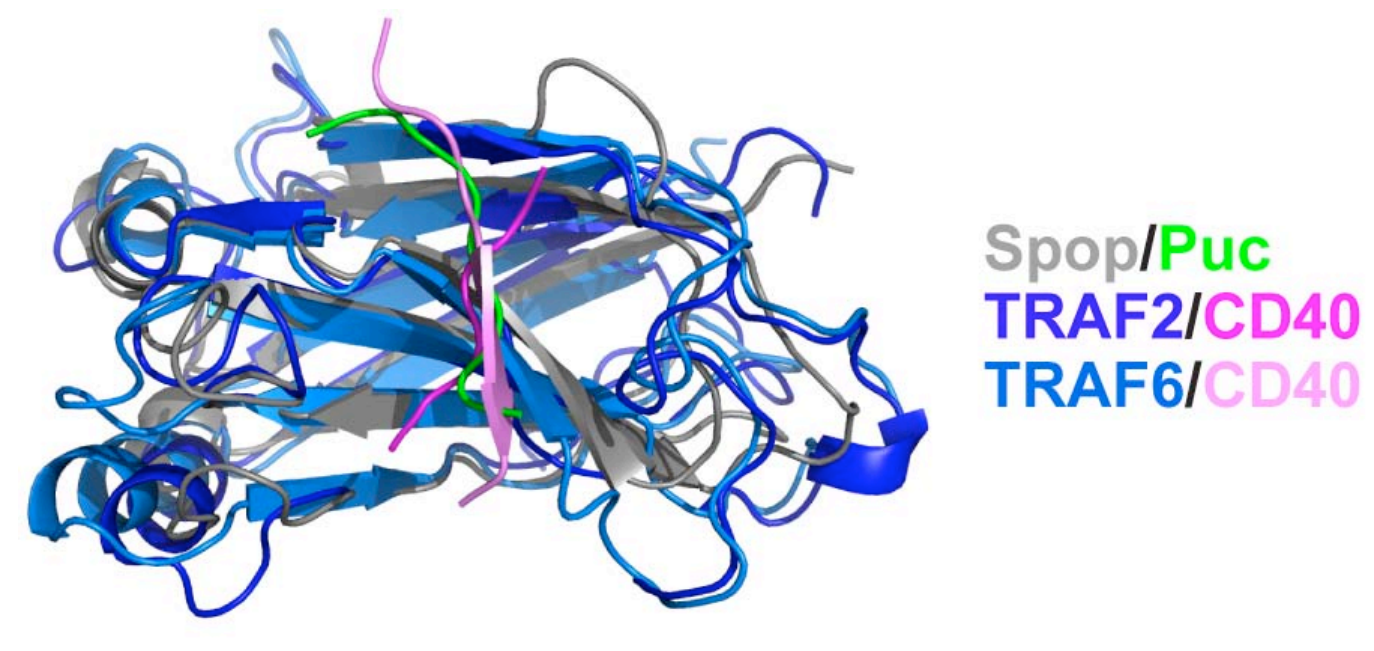

Figure 3.6. Structure superimposition of SPOP ${ }^{\mathrm{MATH}}-\mathrm{Puc}^{\mathrm{SBC} 1}$, TRAF2 ${ }^{\mathrm{TRAF}}-\mathrm{CD} 40$ and TRAF6 ${ }^{\text {TRAF }}$-CD40. MATH/TRAF domains are superimposed with SPOP $^{\text {MATH }}$ in grey and its associated Puc ${ }^{\mathrm{SBC} 1}$ in green, the TRAF2 ${ }^{\mathrm{TRAF}}$ in dark blue and its associated CD40 peptide in magenta (1qsc.pdb), and TRAF $6^{\text {TRAF }}$ in marine and its associated CD40 peptide in pink (1lb6.pdb). 

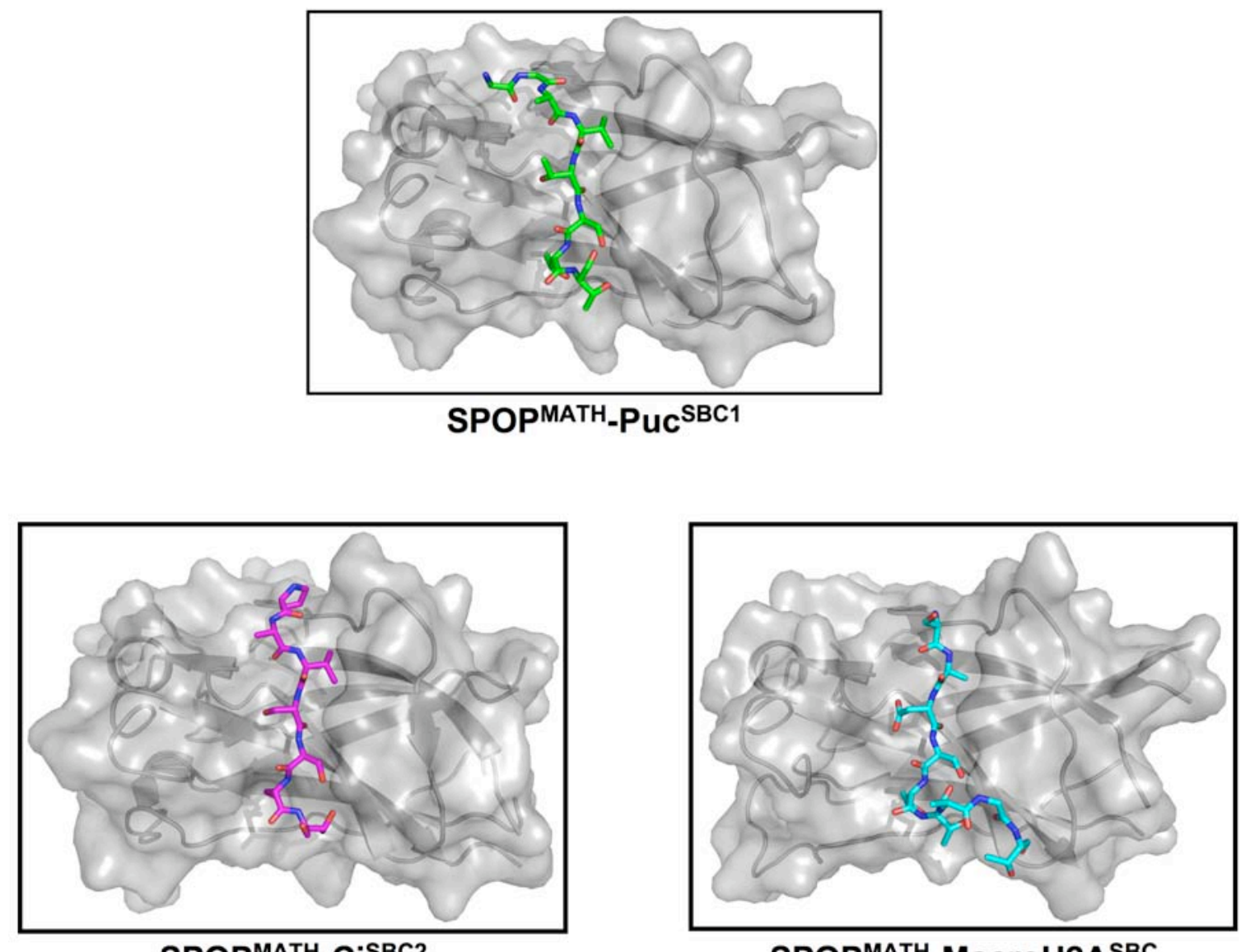

SPOPMATH $_{-} \mathrm{Ci}^{\mathrm{SBC} 2}$ SPOPMATH-MacroH2A ${ }^{\text {SBC }}$

Figure 3.7. Crystal structures of SPOP ${ }^{\mathrm{MATH}}-\mathrm{Puc}^{\mathrm{SBC} 1}, \mathrm{SPOP}^{\mathrm{MATH}}-\mathrm{MacroH}_{2} \mathrm{~A}^{\mathrm{SBC}}$ and SPOP $^{\mathbf{M A T H}}-\mathbf{C i}^{\mathbf{S B C} 2}$. Crystal structures of SPOP ${ }^{\mathrm{MATH}}$ in complex with $\mathrm{Puc}^{\mathrm{SBC1}}$ (green), $\mathrm{MacroH} 2 \mathrm{~A}^{\mathrm{SBC}}$ (cyan), and $\mathrm{Ci}^{\mathrm{SBC} 2}$ (magenta). $\mathrm{SPOP}^{\mathrm{MATH}}$ is represented with a semitransparent surface in grey. 
A MATH/MacroH2A_SBC_pep1

MATH/MacroH2A_SBC_pep2

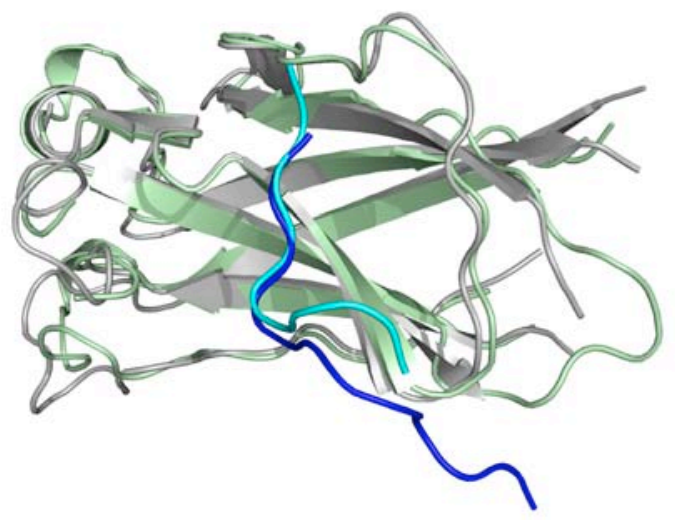

B

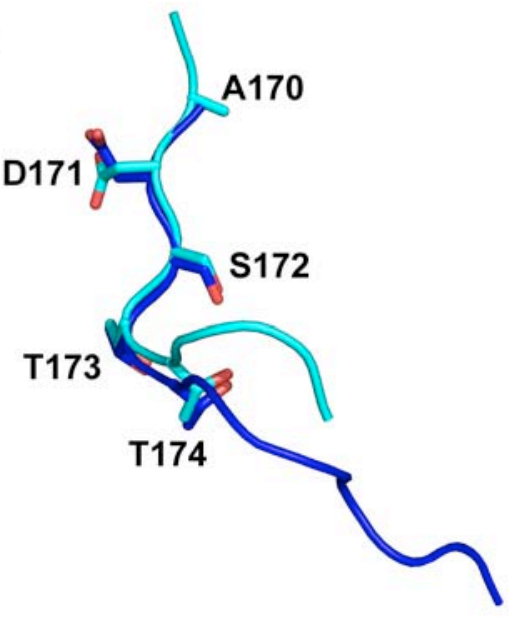

Figure 3.8. Structures of SPOP ${ }^{\mathrm{MATH}}$ complexed with two different peptides corresponding to the MacroH2A SBC. (A) Superposition of co-crystal structures obtained for SPOP ${ }^{\mathrm{MATH}}$ complexes with two different MacroH2A SBC peptides, in distinct crystal forms. The SPOP ${ }^{\mathrm{MATH}}$ (grey) - MacroH2 ${ }^{\mathrm{SBC}}$ complex with peptide 1 (cyan, sequence KAASADSTTEGTPAD, SBC underlined) forms in P6 $6_{5} 22$. The $\mathrm{SPOP}^{\mathrm{MATH}}$ (mint) - MacroH2A ${ }^{\mathrm{SBC}}$ complex with peptide 2 (blue, sequence DSTTEGTPADGFTVL, SBC underlined) forms in C2. While the MATH domain loop regions are flexible, the peptide binding sites are virtually identical. (B) Superposition of the two MacroH2A SBC peptides in the structure with side chains shown in sticks. Although peptide 2 lacks the N-terminal Ala, but the S-S/T-S/T portion of the two peptides superimpose well. 


\subsubsection{Comparison of $\mathrm{SPOP}^{\mathrm{MATH}}{ }_{-} \mathrm{SBC}$ structures}

To compare the recognition of different SBC peptides by SPOP, we superimposed the SPOP ${ }^{\mathrm{MATH}}-\mathrm{SBC}$ structures. Despite the fact that the complexes displayed distinct crystal forms, the SBC-binding sites on SPOP were virtually identical in all the structures, with RMSDs of 0.09-0.19 $\AA$ for the six SPOP residues comprising the bulk of the SBC binding site (including residues Y87, Y123, K129, D130, W131, and F133). The SBC peptides also superimposed well (Fig 3.9). We refer to the positions in the 5 -residue $\phi-\varsigma-\mathrm{S}-\mathrm{S} / \mathrm{T}-\mathrm{S} / \mathrm{T}$ SBC motif as P1-P5 (position 1, etc.).

\section{$\underline{\text { 3.2.2.3 Interactions between } \mathrm{SPOP}^{\mathrm{MATH}} \text { and SBC peptides }}$}

SPOP $^{\mathrm{MATH}}$-SBC interactions were anchored by both hydrophobic and polar interactions. Several bulky hydrophobic residues on SPOP MATH domain, including Y87, Y123, W131 and F133, formed a small hydrophobic cavity. The hydrophobic SBC motif P1 side-chain (Puc V98, MacroH2A A170 and Ci V1362), inserted into this hydrophobic cavity (Fig 3.10A). Similar van der Waals contacts were observed in all three complexes. Eight hydrogen bonds were also observed, mainly involving SBC P2-P5 residues and Y87 and D130 on SPOP (Fig 3.10B, Table 3.1). Sequence flexibility at the $\mathrm{P} 2$ position is explained by the ability of the Y87 hydroxyl to either directly contact an Asp (MacroH2A), or to form water-mediated hydrogen bonds to Thr or Ser (Puc or Ci, respectively). The structures also explain the apparent requirement for a Ser at P3: this side chain both makes hydrogen bonds and is constrained by size within a small cavity formed by side chains of D130, W131 and K129 (Fig 3.10C).

\subsubsection{Mutagenesis Studies Validating SPOP ${ }^{\mathrm{MATH}}$-SBC Interactions}

\subsubsection{Mutations in SBC abolished the interaction between $\mathrm{SBC}$ and SPOP ${ }^{\mathrm{MATH}}$}

To test the significance of the crystallographically observed interactions, we examined the effects of mutations on SPOP ${ }^{\mathrm{MATH}}$ binding to SBC peptides using BIACORE. First, we tested binding to a series of peptides derived from MacroH2A. Abolishing the P1 hydrophobic interaction with a single A170E mutation essentially eliminated binding. Furthermore, substitution of the P3 Ser with bulkier Glu or Trp residues also abolished the interaction, as did substituting the P3-5 STT residues with Alanines (Fig 3.11A).

\subsubsection{Mutations in $\mathrm{SPOP}^{\mathrm{MATH}}$ abolished interactions between $\mathrm{SBC}$ and $\mathrm{SPOP}^{\mathrm{MATH}}$}

Six singly mutated SPOP MATH domains were made for binding analysis and the results were summarized in Fig 3.11B. The D130A and W131A mutants abolished interactions with SBC peptides from all three substrates, supporting the view that these are key residues for substrate recognition. We further tested these two mutants in cellular binding and ubiquitination assays (Contributions of Dr. Jiang Liu, Kevin White's 

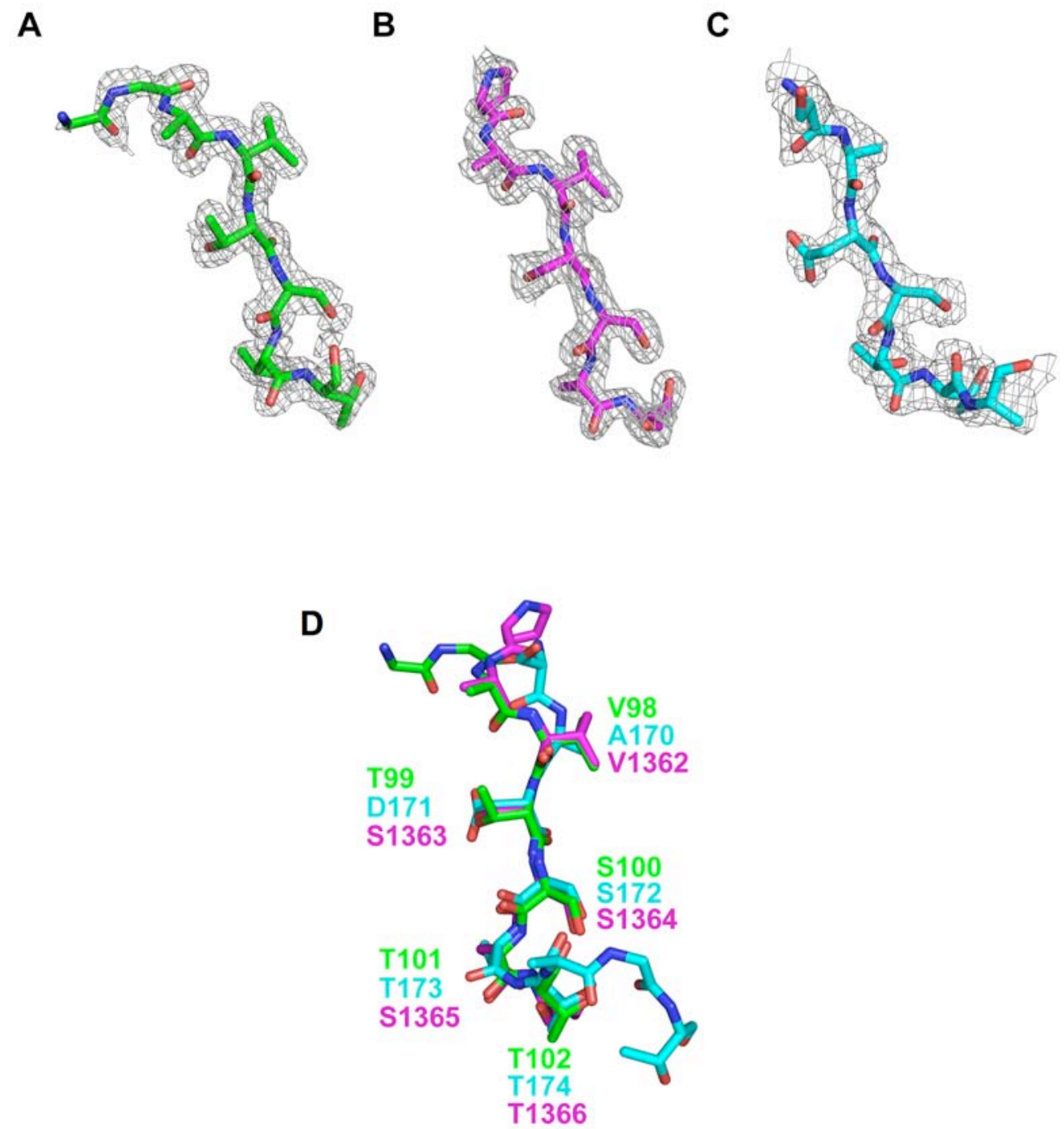

Figure 3.9. Structural comparison of three SBC peptides. (A-C) $2 \mathrm{Fo}-\mathrm{Fc}$ electron density contoured at $1 \sigma$ over SBC peptides from three SPOP ${ }^{\mathrm{MATH}}-\mathrm{SBC}$ complexes. SBC peptides are shown for Puc in green (A), Ci in magenta (B) and MacroH2A in cyan (C), with oxygens in red and nitrogens in blue. (D) Superposition of substrate peptides from the three crystal structures, colored as in A-C. 

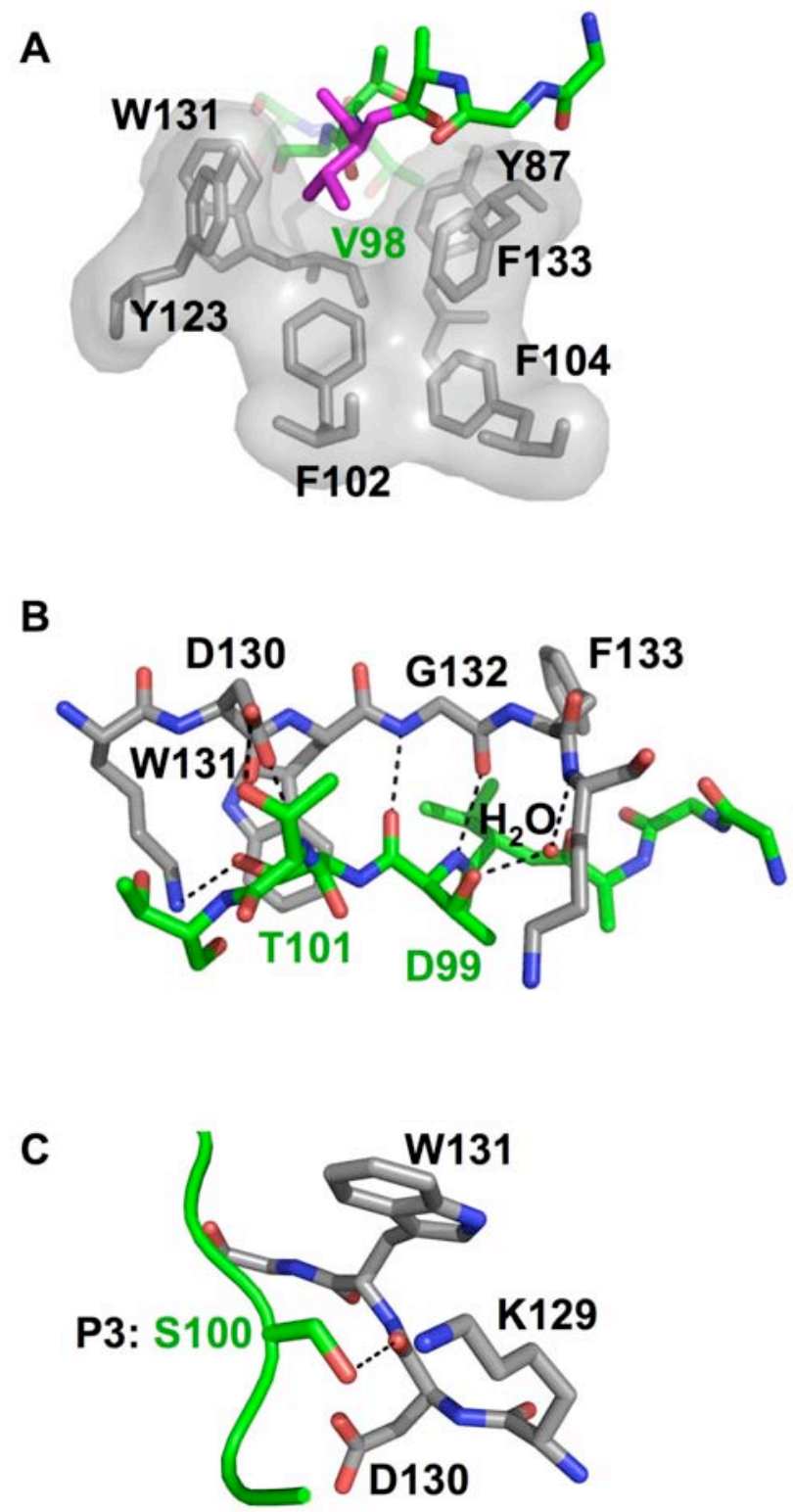

Figure 3.10. Close view of SPOP ${ }^{\mathrm{MATH}}$-SBC interaction. Interactions between $\mathrm{SPOP}^{\mathrm{MATH}}$ and Puc ${ }^{\mathrm{SBC} 1}$ are shown as the example. (A) Close view of Puc ${ }^{\mathrm{SBC} 1} \mathrm{P} 1 \mathrm{Val}$ interacting with $\mathrm{SPOP}^{\mathrm{MATH}}$. $\mathrm{Puc}^{\mathrm{SBC} 1}$ (green) is shown in stick view with $\mathrm{P} 1 \mathrm{Val}$ highlighted with magenta. Residues on SPOP ${ }^{\mathrm{MATH}}$ surrounding Puc ${ }^{\mathrm{SBC} 1} \mathrm{P} 1$ Val are shown as grey sticks. (B) Hydrogen bonds observed between $\mathrm{SPOP}^{\mathrm{MAATH}}$ (grey) and Puc ${ }^{\mathrm{SBC} 1}$ peptide (green) are shown as black dashed lines. The water molecule is shown as a red dot. (C) Close view of SBC P3 Ser interacting with SPOP ${ }^{\mathrm{MATH}}$. Puc ${ }^{\mathrm{SBC}}$ (green) is shown in cartoon with the P3 Ser side chain highlighted in stick view. Residues on SPOP ${ }^{\mathrm{MATH}}$, including K129, D130 and W131 (grey), which surround the SBC P3 Ser are shown as grey sticks, with nitrogens in blue and oxygens in red. The black dashed line indicates a hydrogen bond. 
Table 3.1. Hydrogen bonds between SPOP ${ }^{\mathrm{MATH}}$ and different SBC peptides.

\begin{tabular}{lccc}
\hline \multicolumn{1}{c}{ Interactions } & MATH-MacroH2A & MATH-Puc & MATH-Ci \\
\hline $\begin{array}{l}\text { Between side } \\
\text { chains }\end{array}$ & Y87-D171 & D130-T101 & D130-S1365 \\
$\begin{array}{l}\text { Between back } \\
\text { bones }\end{array}$ & G132 (NH)-D173 & & \\
& G132 (O)-D171 (NH) & G132 (NH)-T99 (O) & G132 (NH)-S1363 (O) \\
$\begin{array}{l}\text { Between side } \\
\text { chain and }\end{array}$ & D130-T173 & D130-T101 & D130-S1365 \\
back bone & K129-T174 & K129-T102 & K129-T1366 \\
$\begin{array}{l}\text { Water } \\
\text { mediated }\end{array}$ & D130-S172 & D130-S100 & D130-S1364 \\
\hline
\end{tabular}



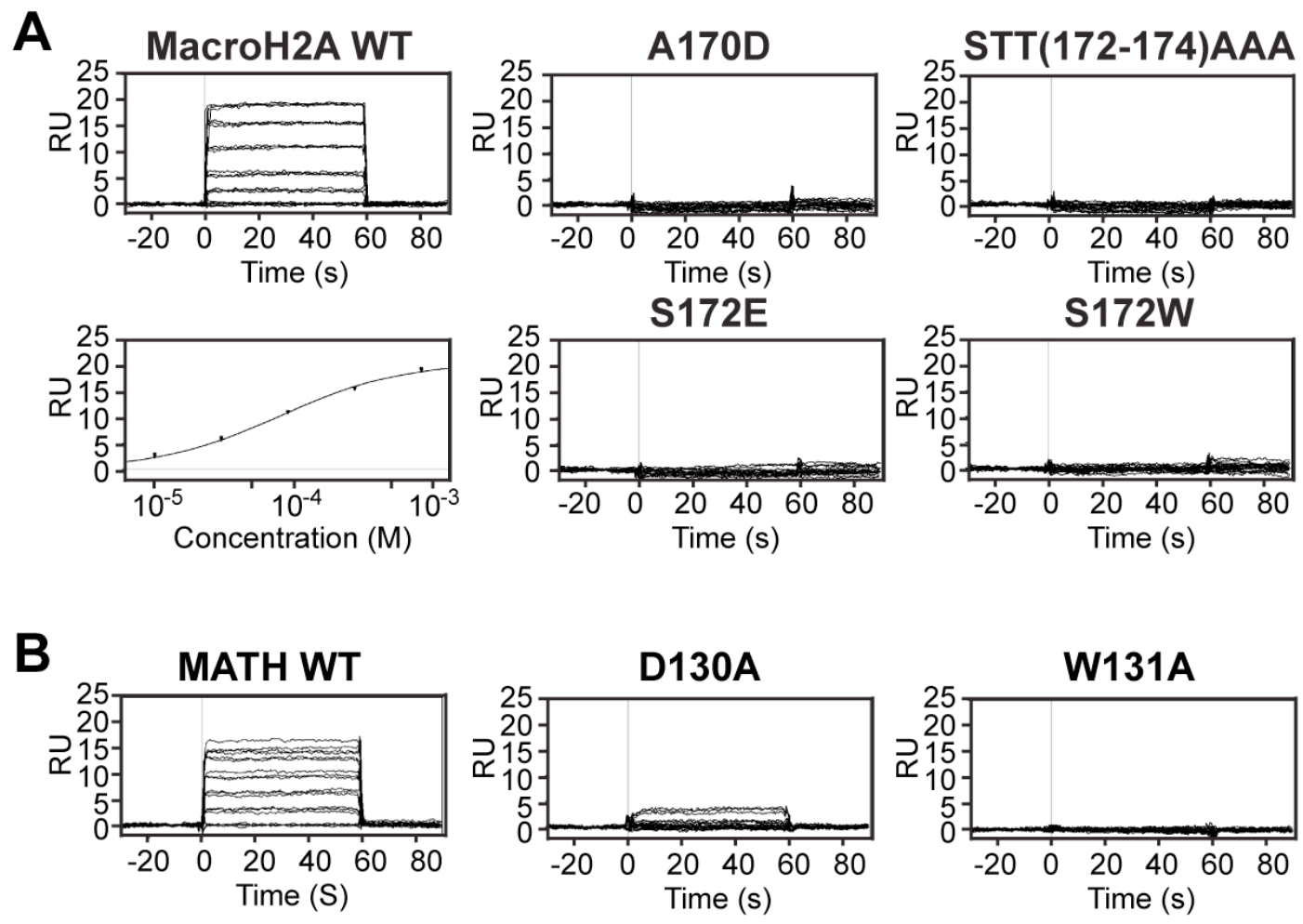

\begin{tabular}{lccc}
\hline & Puc_SBC1 & MacroH2A_SBC & Ci_SBC2 \\
\hline MATH WT & $13.1( \pm .1)$ & $79.9( \pm .3)$ & $105( \pm 1)$ \\
MATH Y87A & $156( \pm 1)$ & $>1000$ & $>1000$ \\
MATH Y123A & $189( \pm 3)$ & $366( \pm 6)$ & $710( \pm 10)$ \\
MATH K129A & $69.4( \pm .9)$ & $325( \pm 3)$ & $188( \pm 2)$ \\
MATH F133A & $129( \pm 5)$ & $>1000$ & $>1000$ \\
\hline
\end{tabular}

Figure 3.11. Validating SPOP ${ }^{\mathrm{MATH}}{ }_{-} \mathrm{SBC}$ structures by binding studies. (A) BIACORE sensograms showing binding of SPOP ${ }^{\mathrm{MATH}}$ to wild-type or indicated mutant versions of the MacroH2 ${ }^{\mathrm{SBC}}$ peptide. The fit used to calculate $\mathrm{Kd}$ for the $\mathrm{SPOP}^{\mathrm{MATH}}$ and $\mathrm{MacroH} 2 \mathrm{~A}^{\mathrm{SBC}}$ interaction is shown on the bottom left. (B) Representative BIACORE sensograms showing binding of wild-type or mutant versions of SPOP ${ }^{\mathrm{MATH}}$ and the $\mathrm{Puc}^{\mathrm{SBCl}}$ peptide. A summary of BIACORE binding data for SPOP mutants and SBC peptides is shown in the bottom table. 
Laboratory, the University of Chicago). In these assays, the SPOP D130A and W131A mutants failed to bind Puc or mediate Puc ubiquitination efficiently (Fig 3.12).

\subsection{DISCUSSION}

\subsubsection{Multiple SBCs on Substrates}

It has been reported that multiple binding sites are required for ubiquitination of substrates, although the mechanism by which this drives ubiquitination is still not fully understood. Studies of the phosphorylation of Sic1 in S. cerevisiae and its binding to $\mathrm{Cul1}^{\mathrm{Cdc} 4}$ provide a good example to demonstrate this complexity mechanism. Sic1 can be phosphorylated at nine different sites, with six or more of them required for highaffinity interaction with the WD40-repeat domain (substrate binding domain) of Cdc4 $[65,101]$. Similar to Sic1, SPOP substrates Ci and Puc contain multiple SBCs. The distances between two SBCs on the same substrate can vary by tens to hundreds of residues away from each other, indicating that they may bind $\mathrm{SPOP}^{\mathrm{MATH}}$ independently.

Multiple SBCs on substrate may provide several advantages. First, it increases the possibility of substrate being recognized by SPOP, thus promoting ubiquitination. Second, if SPOP substrates are modified in vivo, multiple SBC sites ensure that the substrate will still be recognized by SPOP if one or more sites are modified. Third, multiple SBCs may bind a SPOP dimer simultaneously, thus strengthening the interactions between SPOP and substrates (more discussion in Chapter 5).

\subsubsection{General Implications of SBC}

The structure of the SPOP MATH domain closely resembles those of other MATH domains, and like other MATH domains, SPOP associates with its SBC target through a central shallow groove formed by an anti-parallel $\beta$-sheet $[147,151]$. The SBC $\mathrm{P} 1$ hydrophobic residue anchors the interaction, and is bound to a hydrophobic cavity in the SPOP ${ }^{\mathrm{MATH}}$ domain. The SBC sequence from Puc, MacroH2A, and Ci are dominated by hydrophilic residues, which make numerous hydrogen bonds with the SBC binding groove in SPOP ${ }^{\mathrm{MATH}}$. The involvement of SBCs in directing SPOP to its substrates comes from 7 primary observations: 1) SBCs from the substrates Puc and MacroH2A are necessary for binding to SPOP in vitro; 2) peptides corresponding to SBCs from multiple substrates are sufficient for bindig to SPOP in vitro; 3 ) residue substitutions in SBC peptides eliminate binding to SPOP in vitro; 4) mutation of SBCs from Puc lead to the loss of SPOP-dependent ubiquitination in an in vitro; 5) deletion of SBC1 from Puc leads to a loss of binding in cultured cells; 6) mutation of the SPOP SBC binding site abolishes interaction with SBCs from Puc, MacroH2A, and $\mathrm{Ci}$ in vitro, and 7) mutation of the SPOP SBC binding site abolishes binding to and ubiquitination of full-length Puc in cells. While the SBCs we have defined are sufficient for interaction with SPOP ${ }^{\mathrm{MATH}}$, additional interactions may exist between SPOP targets and the MATH domain. 

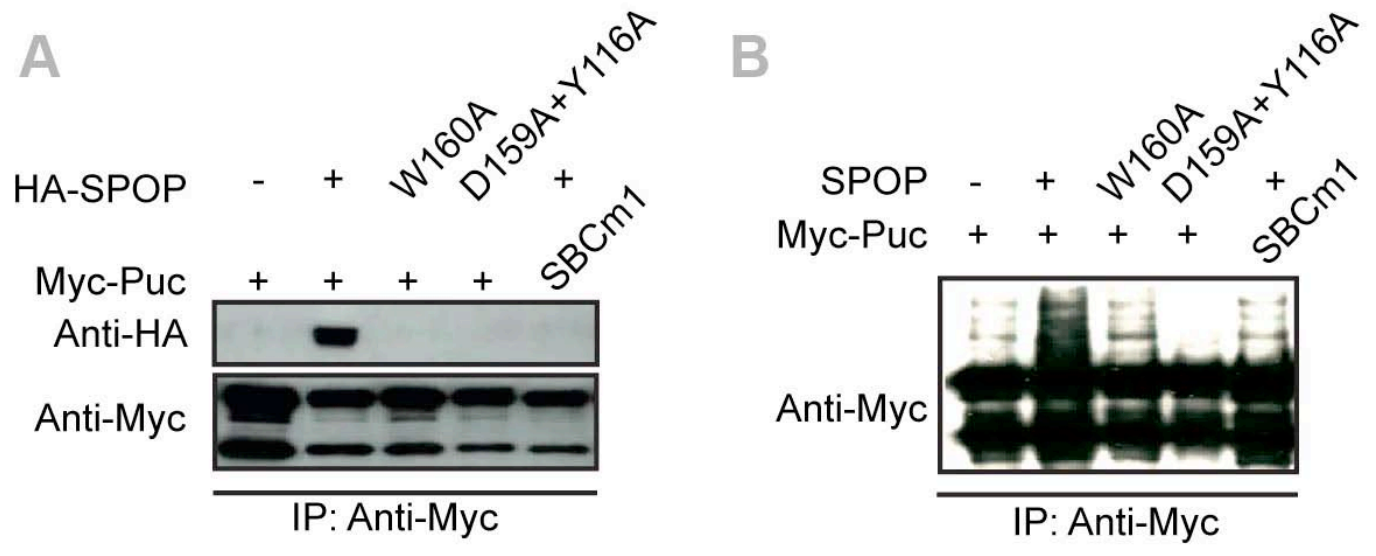

Figure 3.12. Validating SPOP ${ }^{\mathrm{MATH}}$-SBC structures in cells. (A) Western blots showing association of HA-SPOP (top) with Myc-Puc (bottom) or mutants after anti-Myc immunoprecipitation from Drosophila S2 cells co-transfected with the indicated constructs. D130 of human SPOP corresponds to D159 in Drosophila SPOP, and human W131 corresponds to W160. (B) Anti-Myc western blot detecting ubiquitination of Puc from S2 cells co-transfected with wild-type or mutant versions of SPOP. 
Moreover, further studies are required to determine the cellular signaling pathways that make SPOP substrates available for interaction.

Interestingly, the Drosophila SPOP (aka HiB/Roadkill) substrate $\mathrm{Ci}$ is subject to ubiquitination via multiple pathways. In the absence of Hedgehog stimulation, $\mathrm{Ci}$ is ubiquitinated by $\mathrm{SCF}^{\text {Slimb }}$ in a phosphorylation-dependent manner. While many SCF substrates require phosphorylation, there is no evidence that this is the case with the SBC motif. In fact, phosphorylated residues within the SBC motif block the interaction with SPOP (Fig 3.13), raising the possibility that SBC phosphorylation negatively regulates turnover via SPOP. Future studies will be required to dissect the complex interplay between phosphorylation at perhaps a range of sites, and the biochemical decisions between different ubiquitination pathways.

SPOP represents a second BTB protein, in addition to Keap1, for which recognition sequences within substrates are now known. As with SPOP, the Keap1 substrate is also not phosphorylated. Rather, interaction with Keap1 is negatively regulated by post-translational modification. It remains to be seen whether SPOP, its targets, or other BTB proteins are likewise regulated by modifications that prevent their interaction.

Diversification of MATH domains within the context of BTB proteins underlies massive expansion of this protein family in both C. elegans and plants [152]. This expansion has been hypothesized to reflect the use of MATH-BTB proteins antipathogenic agents, directing the turnover of pathogenic proteins. Our results have implications for this expansion and may help explain why MATH-BTB proteins have been co-opted in this way. First, the SBC binding site identified here, which is analogous to ligand-binding sites in other MATH domain proteins, is consistent with the finding that evolutionary drift in expanded MATH-BTB families occurs in this portion of the MATH domain $[119,142,152]$. Thus, this structure appears to be particularly useful in the evolution of novel peptide binding surfaces. Second, flexibility between the MATH and BTB domains may help to accommodate the diverse array of proteins that are likely to be targeted in response to pathogens. In contrast with $C$. elegans and plants, the human genome encodes only two very closely related MATH-BTB proteins, SPOP and SPOPL, the orthologs of Roadkill/HIB. Indeed, the residues that form the SBC binding site are identical in SPOP and SPOPL, suggesting that these proteins may recognize identical substrates, possibly in a redundant manner. Moreover, the analogous SBC binding site in Roadkill/HIB is identical, indicating that the specificity of the Drosophila and human proteins are indistinguishable.

As with the majority of E3s, the identities of substrates for MATH-BTB-Cul3 ubiquitin ligases remain unknown. Our studies suggest that isolated MATH domains can be used to identify peptide sequences that serve as recognition sites for substrates of the MATH-BTB subfamily of Cul3 adaptors. 

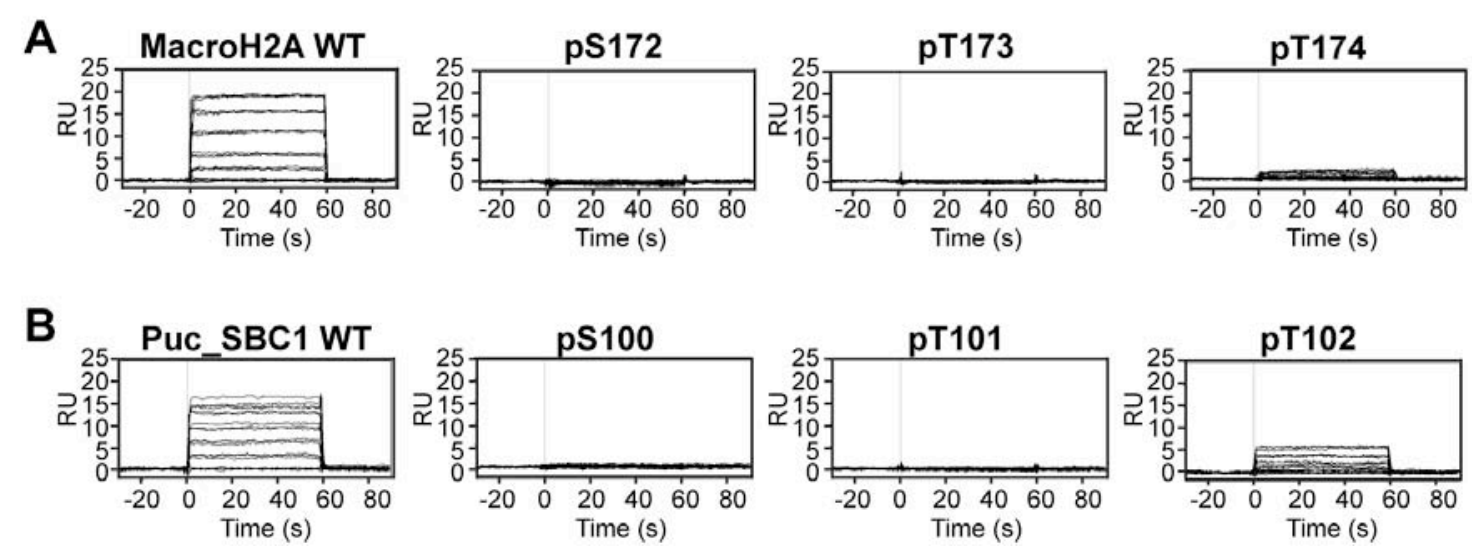

Figure 3.13. SPOP ${ }^{\text {MATH }}$ does not bind phosphorylated SBC peptides. BIACORE sensograms for experiments analyzing binding to either MacroH2 $\mathrm{A}^{\mathrm{SBC}}(\mathrm{A})$ or Puc ${ }^{\mathrm{SBC} 1}$ (B) peptides containing wild type (WT) or the indicated phosphoserine or phosphothreonine substitutions within the SBC sequence. 


\section{CHAPTER 4. STRUCTURE AND FUNCTION ANALYSIS OF SPOP $^{\mathrm{BTB}+}$}

\subsection{INTRODUCTION}

Covalent attachment of ubiquitin via E1-E2-E3 enzyme cascades is a major mechanism for regulating protein function. E3 enzymes, or ubiquitin-protein ligases, are responsible for selecting substrates for ubiquitin modification. The largest subclass of E3 ligases is cullin-RING ligases (CRLs). CRLs are modular, multisubunit enzymes, comprised of interchangeable substrate adaptors dedicated to particular cullin-based catalytic cores [38-40]. A given CRL recognizes its ubiquitination targets through substrate adaptors. Minimally, substrate adaptors contain two functionalities: a protein-protein interaction domain that recruits the substrate, and a domain that recruits the cullin. Substrate adaptors for Cul1, Cul2, and Cul5 utilize two proteins to bind substrate and the cullin: an F-box protein (Cul1) or SOCS-box protein (Cul2 and 5) recruits the substrate; Skp1 and Elongin $\mathrm{C}$ recruit $\mathrm{Cul1}$ or $\mathrm{Cul} 2$ and 5, respectively. The F- or SOCS-box links the substrate-binding domain to Skp1 or Elongin C. By contrast, Cul3 substrate adaptors are single polypeptides, encompassing both a substrate binding domain and a Cul3-binding BTB domain, without an intervening F- or SOCS-box. Thus, BTB proteins appear to merge the functional properties of Skp1 and F-box proteins or Elongin $\mathrm{C}$ and SOCS-box proteins into a single polypeptide chain.

BTB domains were demonstrated to have structural homology with the cullinbinding domain of Skp1 and Elongin C, and to bind Cul3 the way similar to that seen with the Skp1-Cul1, ElonginC-Cul2 interactions [52, 54, 107, 153]. In addition to the Skp1/ElonginC-like core, BTB domains contain additional N-terminal elements that mediate homo- or hetero-dimerization of BTB. BTB-domain containing proteins usually contain additional protein interaction domains, including Zinc-finger (ZF), MATH, Kelch, ankyrin and other poorly characterized domains. MATH-BTB and BTB-Kelch have been implicated in recognition of ubiquitination targets $[4,10,60,61]$.

So far, all known BTB domain structures belong to the BTB-ZF subfamily but the interactions between BTB-ZF and Cul3 are poorly understood. In order to broaden the knowledge of BTB domain and characterize the interactions between BTB protein and $\mathrm{Cul3}$, we focused on two representative BTB proteins, SPOP and Gigaxonin, which are well characterized as Cul3 adaptors. Both SPOP and Gigaxonin were previously shown to bind $\mathrm{Cul} 3$ through the BTB domains. SPOP targets diverse substrates, including Puc, $\mathrm{Ci}, \mathrm{MacroH} 2 \mathrm{~A}$ and Daxx, for ubiquitination in different signaling pathways $[4,58,116$, 122]. Gigaxonin, the mutations of which cause fatal neurodegenerative disorder giant axon neuropathy [154], controls degradation of MAP1B, which is critical for neuronal survival $[60,155]$. SPOP and Gigaxonin belong to MATH-BTB and BTB-Kelch subfamilies respectively. The BTB domain of SPOP (residues 172-297) is located at the $\mathrm{C}$ terminus and followed by nuclear localization sequence (NLS, residues 360-374). By contrast, the BTB domain is located at the $\mathrm{N}$ terminal of Gigaxonin (residues 1-126). In 
spite of different domain arrangement, both SPOP and Gigaxonin dimerize through the BTB domains.

Here we report crystallographic and biochemical characterization of SPOP BTB domain and its interactions with Cul3. We describe a novel Cul3-binding domain, the 3-box, conserved in Gigaxonin and among other BTB substrate adaptors as a C-terminal extension of BTB domain. These findings reveal that the Cul3-binding domain structurally resembles a fusion of Skp1 and F-box, and give insight into the evolution of cullin substrate adaptors.

\subsection{RESULTS}

\subsubsection{Definition of SPOP ${ }^{\mathrm{BTB}+}$}

Beyond the $\mathrm{C}$ terminus of SPOP MATH domain, there are two function groups: BTB domain (residues 190-297) and nuclear localization sequence (residues 360-374). We identified a well-behaved protein component containing residues 172-337 for crystallographic and biochemical studies. This protein component comprises BTB domain and additional 40 amino acids. In this study, we refer to it as $\mathrm{SPOP}^{\mathrm{BTB}}$.

\subsection{2 $\mathrm{SPOP}^{\mathrm{BTB}+}$ Mediated Dimerization of Cul3}

In general, BTB domains form homo- or hetero-dimers and bind the $\mathrm{Cul} 3$ $\mathrm{N}$-terminal domain $\left(\mathrm{Cul}^{\mathrm{NTD}}\right)$ in a manner resembling Skp1 interacting with Cul1 [45, 56]. In order to understand whether $\mathrm{SPOP}^{\mathrm{BTB}+}$ maintains its dimeric structure in the $\mathrm{SPOP}^{\mathrm{BTB}+}-\mathrm{Cul}^{\mathrm{NTD}}$ complex, we studied the stoichiometry of $\mathrm{SPOP}^{\mathrm{BTB}+}$ binding to $\mathrm{Cul}^{\mathrm{NTD}}$. First, we validated $\mathrm{SPOP}^{\mathrm{BTB}+}$ binding $\mathrm{Cul}^{\mathrm{NTD}}$ in vitro by native gel shift assay and analytical gel filtration chromatography (Fig 4.1A, B). Then we examined the stoichiometry of $\mathrm{SPOP}^{\mathrm{BTB}+}-\mathrm{Cul} 3^{\mathrm{NTD}}$ complex by analytical ultracentrifugation (AUC) (Fig 4.1C). Our data indicate that $\mathrm{SPOP}^{\mathrm{BTB}+}$ forms a 2:2 complex with the $\mathrm{Cul}^{\mathrm{NTD}}$, consistent with prior data indicating that BTB-Cul3 complexes are oligomeric in vivo [41, 89].

\subsubsection{The Skp1/ElonginC-like Core Domain of SPOP ${ }^{\mathrm{BTB}+}$}

To better understand SPOP-Cul3 interaction, I determined a crystal structure of the SPOP BTB+ domain. In the SPOP ${ }^{\mathrm{BTB}+}$ crystal structure, residues 172-297 displayed overall similarity to Cul1, Cul2/5 adaptors Skp1 and Elongin C (Fig 4.2), which consist of a short three-strand $\beta$ sheet and a cluster of five $\alpha$-helices. Similar to the BTB domain structures of the BTB-ZF family, SPOP displayed the BTB-specific N-terminal extension, which mediates dimerization. Hydrophobic residues on the N-terminal helix were lined up along the dimer interface, suggesting strong hydrophobic interactions 
A

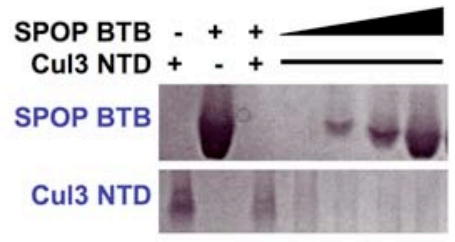

SPOP BTB / Cul3 NTD

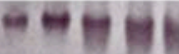

B

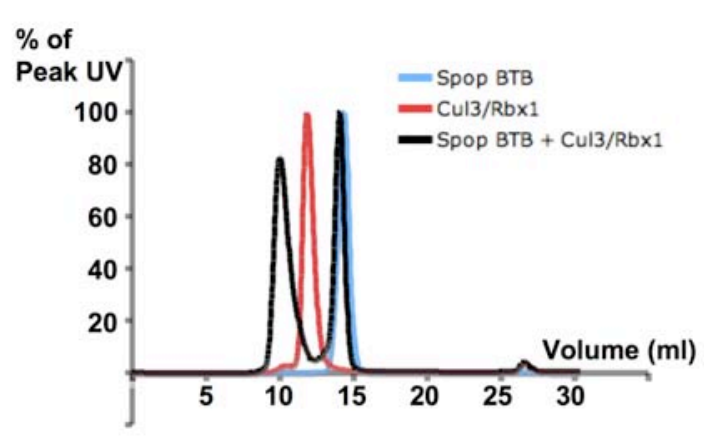

C
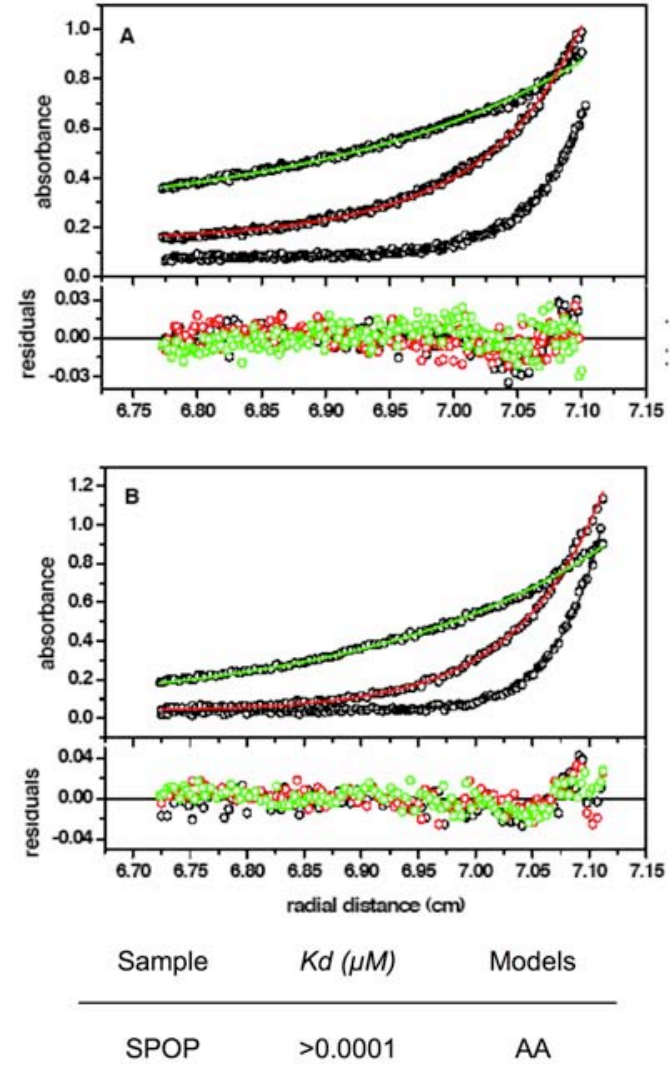

Cul3-SPOP $\operatorname{Kd}(\mathrm{AB})=0.10$ $\operatorname{Kd}(\mathrm{AA})=0.008$

$B(A) B$

Figure 4.1. $\mathrm{SPOP}^{\mathrm{BTB}+}$ mediates dimerization of Cul3. $\mathrm{SPOP}^{\mathrm{BTB}+}$ is labeled as SPOP $\mathrm{BTB}$ in this figure. (A) $\mathrm{SPOP}^{\mathrm{BTB}+}$ and $\mathrm{Cul}^{\mathrm{NTD}}$ form a protein complex in native gel shift assay. Proteins are stained with coomassie blue. (B) $\mathrm{SPOP}^{\mathrm{BTB}+}$ and $\mathrm{Cul}^{\mathrm{NTD}}$ form a complex in analytical gel filtration experiment. (C) Equilibrium analytical ultracentrifugation data for interactions between $\mathrm{SPOP}^{\mathrm{BTB}+}$ dimer and $\mathrm{Cul}^{\mathrm{NTD}}$. Kd values and fitting models are shown in the bottom table. 


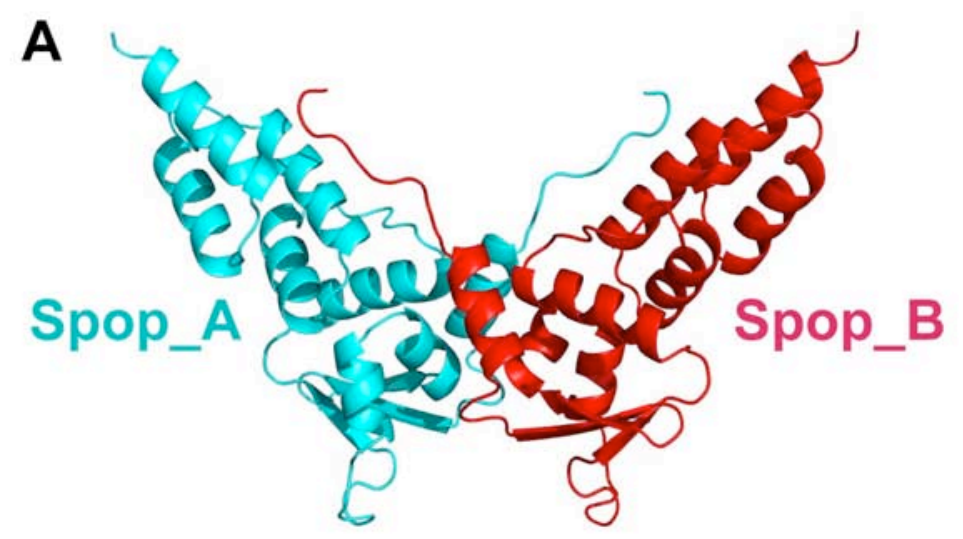

B
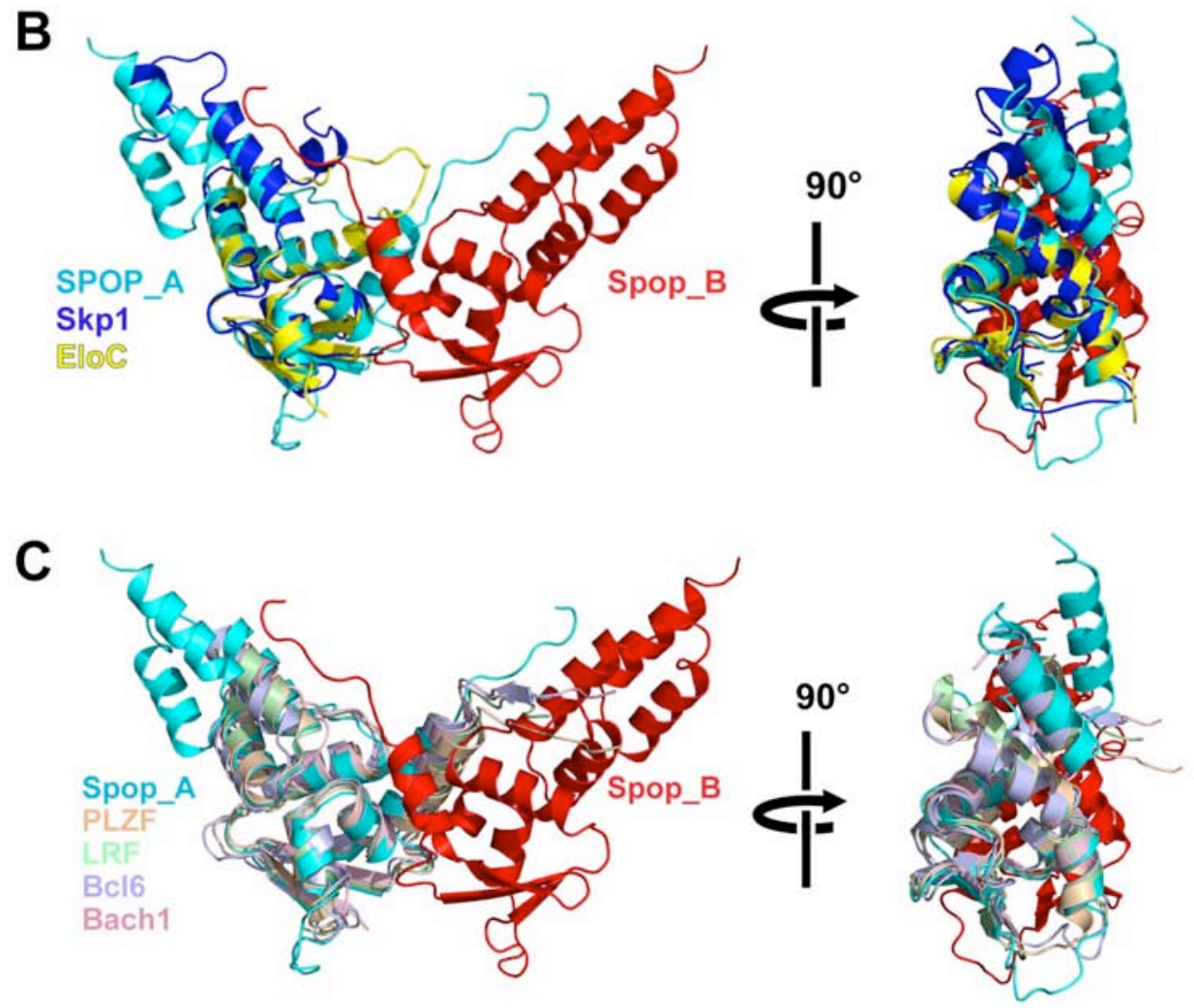

Figure 4.2. Structural superimposition of $\mathrm{SPOP}^{\mathrm{BTB}+}$, Skp1, EloC and other BTB domains. (A) Crystal structure of $\mathrm{SPOP}^{\mathrm{BTB}+}$. One molecule of the dimeric $\mathrm{SPOP}^{\mathrm{BTB}+}$ structure is colored in cyan (SPOP_A) and the other is colored in red (SPOP_B). (B) Two views, rotated $90^{\circ}$ in y, showing structural alignment of $\mathrm{SPOP}^{\mathrm{BTB}}{ }^{\circ}$, Skp1 and EloC. Skp1 is shown in blue and EloC in yellow. (C) Two views, rotated $90^{\circ}$ in y of one molecule of the BTB dimer from PLZF (tan, 1buo.pdb), LRF (pale green, 2nn2.pdb), Bcl6 (light blue, 1r29.pdb), and BACH1 (light pink, 2ihc.pdb) was aligned to SPOP ${ }^{\text {BTB+ }}$ molecule "A" (cyan). 
between each BTB monomer (Fig 4.3). In the AUC analysis of $\mathrm{SPOP}^{\mathrm{BTB}+}$, monomeric $\mathrm{SPOP}^{\mathrm{BTB}+}$ is hardly detected (Fig 4.1C), which is consistent with structure observations.

\subsubsection{The 3-box: a Cul3-Binding Element}

Unexpectedly, the SPOP ${ }^{\mathrm{BTB}+}$ displays an additional structural feature: a pair of C-terminal $\alpha$-helices (Fig 4.2). To gain insight into potential functions of these helices, we compared the SPOP ${ }^{\mathrm{BTB}+}$ structure with Skp1-F-box and ElonginC-SOCS-box in the context of cullin binding complexes. The SPOP helices are located at the position corresponding to an F-box or SOCS-box in other CRLs (Fig 4.4). Notably, the F-box was shown previously to contact Cul1 in the prior Cul1-Skp1-Skp2Fbox crystal structure [45], and the SOCS box has also been implicated in Cul2 and Cul5 binding [50]. Thus, we tested whether the SPOP helices have a role in Cul3 binding. Deletion of these two helixes decreased interactions between SPOP and Cul3 (Fig 4.5). Thus, due to functional parallels to an F-box or SOCS-box in cullin binding, we refer to this structure as the 3-box, for Cul3-interacting box, and we redefine our BTB+ construct as BTB-3-box.

\subsubsection{Structural Conservation of the 3-box}

A key feature of F-boxes and SOCS-boxes is that they are conserved among Cull and $\mathrm{Cu} 2 / \mathrm{Cul5}$-adaptors, which display a range of substrate-binding protein interaction domains. Thus, to determine whether the 3-box is conserved, we set out to study the corresponding structure of another Cul3-binding BTB protein. We obtained crystals for an engineered fragment corresponding to residues 1-258 of human Gigaxonin (Gig), a BTB-Kelch Cul3 adaptor. As with other BTB-Kelch proteins, the Gig BTB domain is located N-terminal of the substrate-binding domain, and thus displays a significantly divergent domain arrangement from SPOP (Fig 4.6A). The overall Gig structure is superimposed well on to SPOP ${ }^{\mathrm{BTB} / 3 \text {-box }}$ (C $\alpha$ RMSD 2.06 ) (Fig 4.6B), and the 3-boxes of both proteins display virtually identical backbone structures (C $\alpha$ RMSD $0.39 \AA$ ) despite their distinct sequences (Fig 4.6C). The sequence alignment of the corresponding 37 amino acids following BTB domains from five $\mathrm{Cul3}$ adaptors and several uncharacterized BTB proteins reveals conservation (Fig 4.7), suggesting that the 3-box may also exist in Keap1, KLHL12 and Actinfilin. As most of the conserved residues are buried and mediate inter-helical packing, it is possible that $\mathrm{Cul} 3$ recognizes a range of sequences with a common 3-box tertiary structure.

\subsubsection{Predicted 3-box in a Subset of BTB Proteins}

Only a handful of BTB proteins have been explicitly shown to mediate ubiquitination. As we found two of these to contain a 3-box, we attempted to identify potential 3-boxes in other BTBs. We performed a bioinformatics study to search for a paired helix structure within 40 amino acids beyond the $\mathrm{C}$ terminus of BTB domains from 183 human BTB proteins. Our analysis predicts that like SPOP and Gig, all 

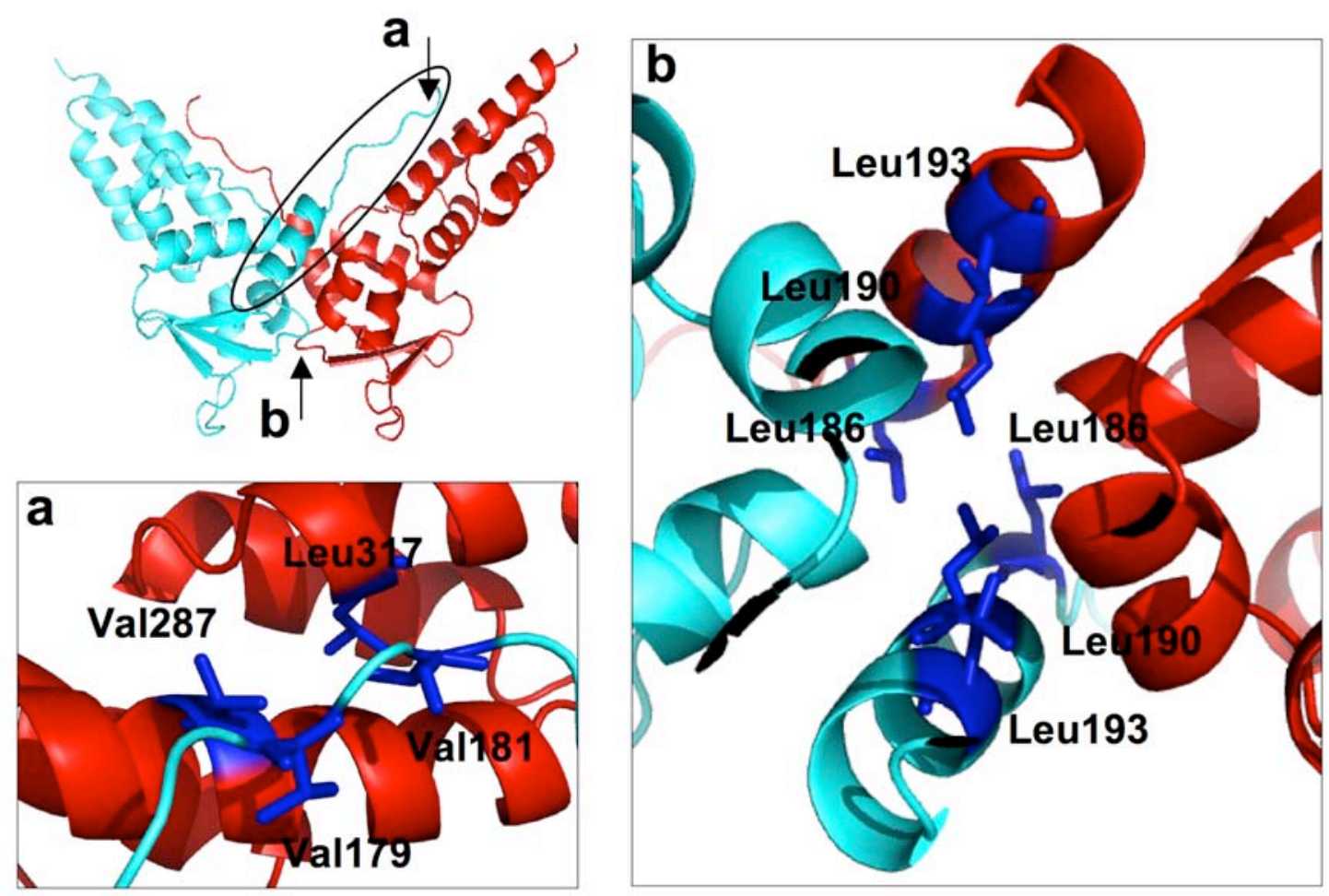

Figure 4.3. Close view of SPOP${ }^{\mathrm{BTB}+}$ dimer interface. Two regions (a and b) on $\mathrm{SPOP}^{\mathrm{BTB}}{ }^{+}$involved in dimerization are enlarged individually. One molecule of the dimeric $\mathrm{SPOP}^{\mathrm{BTB}+}$ is colored in cyan and the other is colored in red. Hydrophobic residues are highlighted in blue as sticks. 

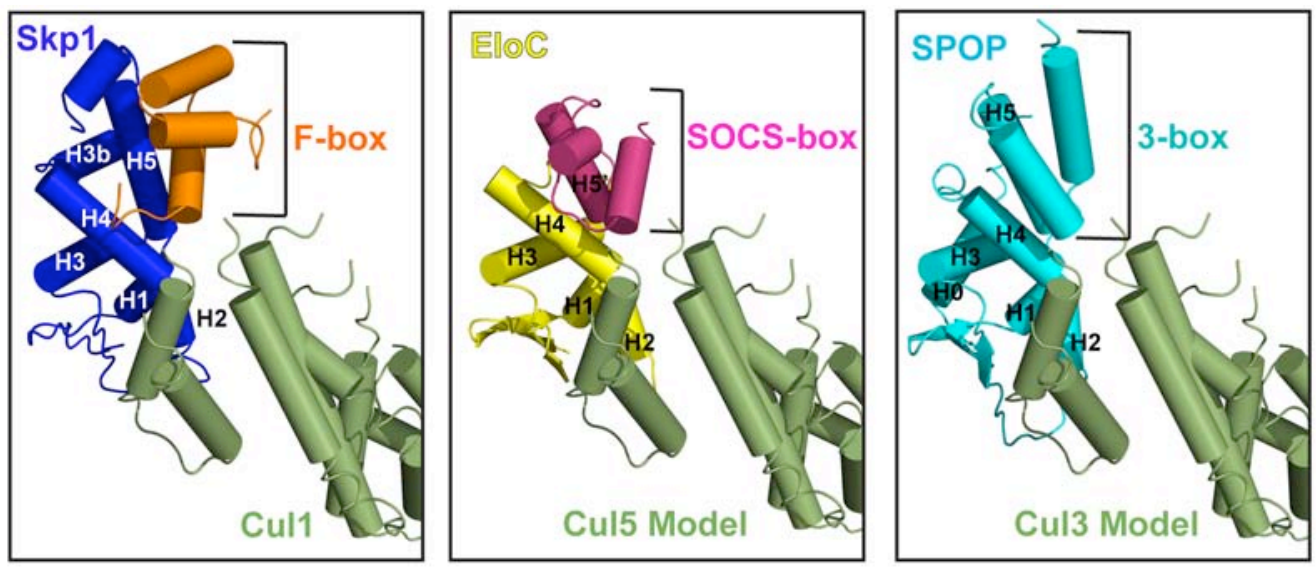

Figure 4.4. Identification of 3-box from $\mathrm{SPOP}^{\mathrm{BTB}+}$ crystal structure. Structural comparison of the Skp1 (blue) -F-box ${ }^{\text {Skp2 }}$ (orange) -Cul1 (green) structure (1LDK.pdb), the Elongin $\mathrm{C}$ (yellow) - SOCS-box ${ }^{\mathrm{VHL}}$ structure (1VCB.pdb) docked onto a structural model of Cul5 (green), and the BTB+ domain of SPOP docked on a structural model of $\mathrm{Cul3}$ (green). The relative locations of the SPOP 3-box, F-box, and SOCS-box are indicated. EloC-SOCS-box-Cul5 and BTB/3-box-Cul3 models were made using the Skp1-F-box-Cul1 crystal structure as the template. 

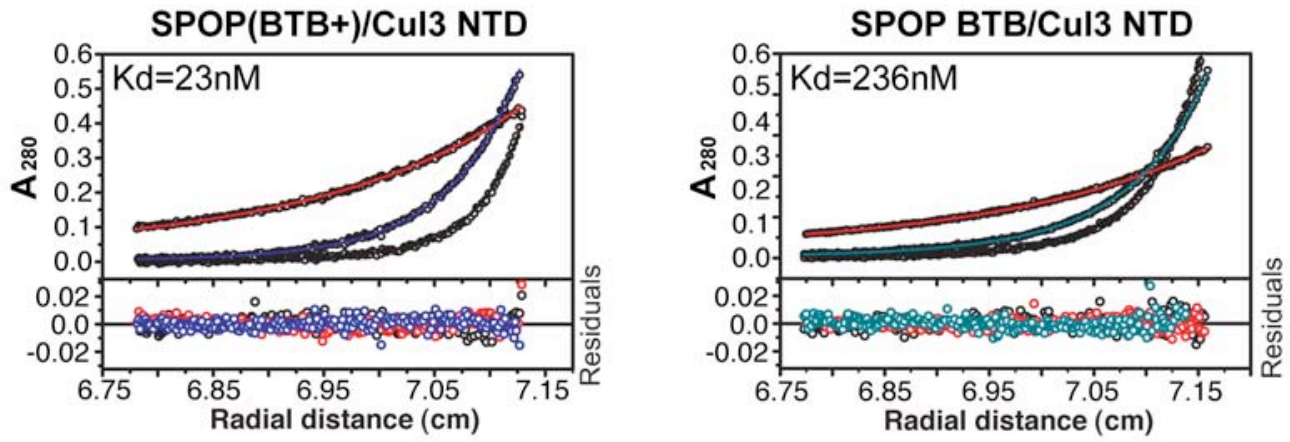

Figure 4.5. 3-box contributes $\mathrm{Cul3}$ binding. Equilibrium analytical ultracentrifugation data for interaction between SPOP ${ }^{\mathrm{BTB}+}$ and SPOP ${ }^{\mathrm{BTB}}$ (lacking the 3-box) and the $\mathrm{N}$-terminal domain of $\mathrm{Cul3}$. Kd values are shown for $\mathrm{Cul}^{\mathrm{NTD}}$ interaction with the indicated SPOP variant. 


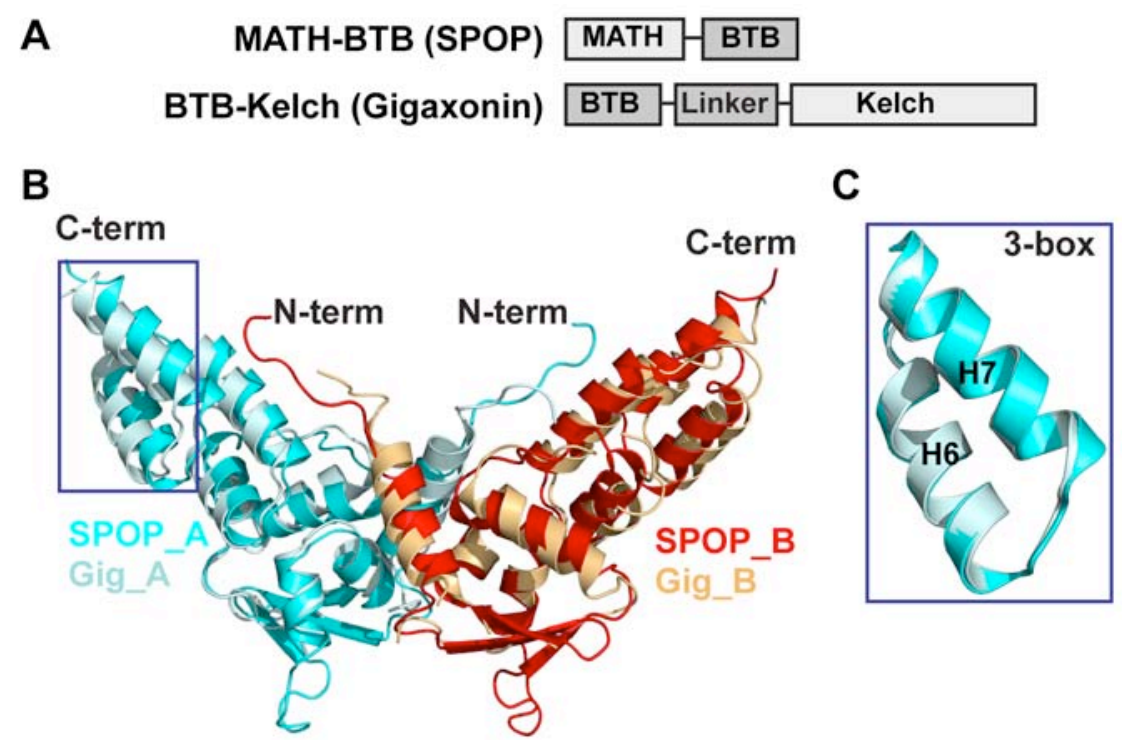

Figure 4.6. The 3-box structure is conserved in another BTB subfamily. (A) Schematic view of domain arrangement of SPOP and Gigaxonin, which represent two distinct BTB subfamilies, MATH-BTB and BTB-Kelch, respectively. (B) Overall structural alignment of SPOP ${ }^{\mathrm{BTB} / 3 \text {-box }}$ and Gigaxonin ${ }^{\mathrm{BTB} / 3 \text {-box }}$. Residues C-terminal of the Gigaxonin 3-box were omitted for clarity. (C) Superposition of the SPOP and Gigaxonin 3-boxes. SPOP ${ }^{3-b o x}$ is in cyan and Gigaxonin ${ }^{3-b o x}$ is in light blue. 


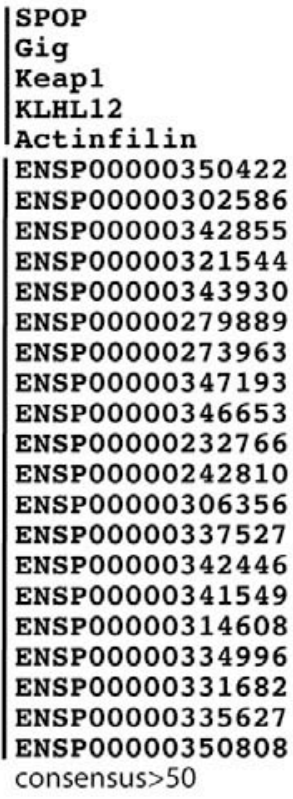

120303040

NLSVENAAEILI LADLHSADQLKTQAVDFINYHASDVLET. . C IAAENCIGIRDF A LHY CLHHVHYLATEYLETHFRDVSST. . QL DP SNA IGIANFAEQIGCVELHQRAREY IYMHFGEVAKQ... QLDPSNCLGIRDFAETHNCVDLMQAAEVF SQKHFPEVVQH. . QLDPSNCLGIRGFADAHSCSDLLKAAHRYVLQH FVDVAKT. . . Q QVGNCL QVMWLA DR H D PELYTAAKHCAKTH LA L LNTEE . . NAENCVRLLSFADLFSCEELKQSAKRMVEHKFTAVYHQDA. . NLVNCLQLLS IS DS Y GS TS LF DHAL HFVQH F SLLF K S D . .. SLANCYEVLSAAKRQRLNELRDAAYCFMSDHYLEVLREPA. ...P SNCLGIRGFADAHSC SDLIKAAHRY VLQH FVDVAKTEE F ...PS NCLGIRAFADA Q GC I L L KVAHSYTMEN IMEVIRNQEF $\ldots$ PSNCLAVRAFAESHNR I L MDMADQYACDHFTEVVECEDF $\ldots$ PGNCIGISRFAETYGCRDLYLAATKY ICONFEAVCOTEEF ...P L N C IGIF QF S EQ I ACHDLLEFS ENY I H VH F LEVH S GEE F . H P KNCL GVRQFAETMMCAVLYDAANSF IHQH FVEVSMSEE . . DPCNCLGIQRFADTHSLKTLFTKCKNFALQTFEDVSQHEE . . H P TN C L GMLLLS D A QC T K LYELSWRMCLSN F T IRKNED . . DLANCL DMQDFAEA F C SGLASAAQRF I LRHVGELGAEOL . ...VTSCISYRNFASCMGDSRLLNKVDAY I QEHLLQ ISEEEEF . S PARCLAL F P MAEA PGLERLWSKARHYL L TH L PAVA L CPA. .. H L SNCVAIH S LAHMY TLS Q LA LKA A DM IRR NFHKVIQDEE. . C CRANCLRYLFLAELFELKEVS DVAYSG IRDNFHYWASPEG . $\ldots$ EEN I L DVYRLAELF DLSRLTEOLDTY I L KNFVAFSRTDKY ... SFNYLDLYRLADLFNLTLLEKAVIDFLVKHLSELLKSRPE . SVQNYKQVCKIAALH GLEETKKLAN KY LVEDVLLLNFEEM . ....ncl.i.fad....el...a. . yi.hf.ev...e.

Figure 4.7. 3-box sequences in other BTB proteins. Sequence alignment of 37 amino acids corresponding to 3-boxes from 5 Cul3-interacting BTB proteins (SPOP, Gig, Keap1, KLHL12 and Actinfilin) and 20 un-characterized BTB proteins. The output was generated with ESpript. 
MATH-BTB and BTB-Kelch protein have a helix pair following the BTB domain, which may function as a 3-box. By contrast, we identified potential 3-box structures in only a subset of the BTB-ZF, T1-like BTB, and other BTB proteins (Fig 4.8). It is possible that BTB proteins lacking obvious 3-box may not function as $\mathrm{Cul} 3$ adaptors, or they may utilize alternative mechanisms to strengthen their interaction with Cul3.

\subsection{DISCUSSION}

The BTB domain, which can bind Cul3, shares a common molecular fold with the Cul1 and Cul2/5 binding proteins, Skp1 and Elongin C. Through our crystallographic analysis of $\mathrm{SPOP}^{\mathrm{BTB}+}$, we found that sequences C-terminal to the canonical BTB-fold adopt a 2-helix bundle structure reminiscent of that seen with F-box and SOCS-box structures. As with the F-box and SOCS-box motifs, which interact with Cull and $\mathrm{Cul} 2 / 5$, respectively, we found that this 2-helix bundle increases the affinity of $\mathrm{SPOP}^{\mathrm{BTB}+}$ for Cul3. Therefore, we refer to this structural feature as the 3-box, to denote its interaction with Cul3. It is tempting to speculate an evolutionary relationship between F-box proteins, SOCS-box proteins, and BTB-proteins, although at this point it is unclear whether the functional similarities are a consequence of convergent evolution, or divergence from a common ancestor. In the case of divergent evolution, a BTB may represent the primordial CRL adaptor, encompassing Skp1, F-box, and substrate-binding domain in a single polypeptide.

The SPOP BTB domain and 3-box are near the C terminus. In contrast, the majority of BTB domains are located at the N-terminus, followed by a linker sequence (in some cases referred to as the BACK domain) and substrate binding domains. Our data also reveal the 3-box in the N-terminal BTB domain configuration, as sequences C-terminal to the canonical BTB domain of Gigaxonin also form a 2-helix bundle that superimposes onto that of the SPOP 3-box. The residues that form the core of the 3-box are conserved, not only between SPOP and Gigaxonin, but also among a variety of BTB-Kelch proteins, suggesting that the 3-box coevolved as part of an extended BTB domain for Cul3 binding.

The 3-box might be a marker for Cul3-interacting BTBs. As more and more BTB proteins are studied, many have been characterized as Cul3-binding proteins, mediating the ubiquitination of specific substrate proteins. The list of human BTB proteins that interact with Cul3 has increased to ten, including SPOP [116], Gigaxonin [60], Keap1 [156], KLHL9 [59], KLHL12 [10], KLHL13 [59], KCTD5 [111], BPOZ-2 [157], RhoBTB [90] and Actinfilin[158]. However, It is unknown if all BTB proteins function as bona fide Cul3 adaptors. In fact, Harper's laboratory (Harvard Medical School) has identified a BTB protein that does not associate with Cul3 (unpublished data). It's still not clear why some BTBs bind Cul3 while others don't, despite conserved BTB folding. Our studies of 3-box predict the existence of 3-box among the ten identified Cul3-binding BTBs, but not in most BTB-ZF transcription factors and T1-like BTB proteins. Thus, we speculate that BTB proteins might require 3-box to function as Cul3 adaptors. In this 
Human BTB subfamilies:
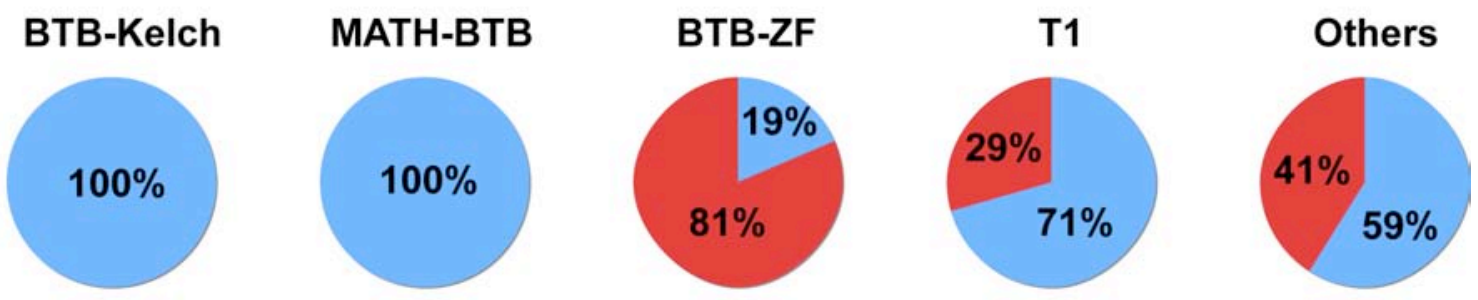

With extra helices after BTB (predicted)

No extra helices after BTB (predicted)

Figure 4.8. Identification of 3-box in a subset of BTB proteins. Pie chart showing percent of members of the different BTB protein subfamilies from humans predicted to have a 3-box (blue). 
sense, 3-box might be used as the marker for Cul3-interacting BTBs to help identify more Cul3- interacting adaptors.

The BTB domain of SPOP adopts a dimeric structure, which superimposes on a number of BTB domains whose structures have been previously determined. Through a series of biochemical experiments, we have found that the dimeric SPOP BTB domain can assemble with 2 molecules of $\mathrm{Cul3}$, thereby generating a dimeric ubiquitin ligase containing two catalytic cores. BTB domain mediated $\mathrm{Cul} 3$ dimerization is different from previously identified F-box protein-dependent $\mathrm{Cull}$ dimerization. $\mathrm{SPOP}^{\mathrm{BTB}+}$ and $\mathrm{Cdc} 4^{\text {D-domain }}$ ( $\mathrm{D}$ domain is the dimerization domain in F-box proteins) have distinct tertiary structures (Appendix A, Fig A.5). Dimerization of CRLs may function in multiple ways to affect substrate ubiquitination (will be discussed in detail in Chapter 5). A common feature of Cul1 CRL and Cul3 CRL dimmers is multiple E2 docking sites, which potentially can increase the local concentration of Ub-loaded E2, thus enhancing the efficiency of CRLs. 


\section{CHAPTER 5. PUTTING THE MATH AND BTB DOMAINS TOGETHER: INSIGHTS INTO BTB-CUL3 UBIQUITIN LIGASES}

\subsection{INTRODUCTION}

Cullin RING ubiquitin ligases (CRLs) are modular multi-subunit protein complexes. The BTB-Cul3 family is the largest CRL subclass, with 190 BTB proteins in humans. BTB proteins are grouped into eight categories, defined by the nature of the substrate-binding protein-protein interaction domains. The MATH-BTB module, in which a MATH domain is N-terminal of the BTB domain, is the largest BTB family, and is also one of the most abundant among all proteins. Despite the broad importance of MATH-BTBs, it is not known how any MATH-BTB protein bridges a substrate and Cul3 for ubiquitination. The best-studied mammalian MATH-BTB Cul3 adaptor is SPOP. SPOP is conserved among higher eukaryotes and functions in diverse signaling pathways, by mediating ubiquitination of divergent substrates. SPOP negatively regulates hedgehog pathway by mediating ubiquitination and degradation of $\mathrm{Ci}$, which is essential for normal eye development $[4,125,126]$. Stable X chromosome inactivation involves ubiquitination of histone MacroH2A by Cul3 ${ }^{\text {SPOP }}[122,123]$. SPOP also targets Daxx for ubiquitination and degradation to regulate Daxx-mediated cellular processes, including transcriptional regulation and apoptosis $[116,124]$. The molecular sizes of SPOP substrates vary, ranging from $45 \mathrm{kDa}$ to $160 \mathrm{kDa}$. In spite of the numerous important pathways regulated by SPOP, the molecular basis for multi-size substrate engagement by a single $\mathrm{Cul3}{ }^{\mathrm{SPOP}}$ ligase remains a mystery.

SPOP, as with other BTB proteins, has three functionalities within two domains: binding substrate through MATH domain and binding Cul3, dimerization through BTB domain. Dimerization is a shared feature of some CRLs - some F-box proteins dimerize via a "D-domain", which is structurally distinct from a BTB domain. Currently, two models have been proposed regarding the role of F-box protein dimerization in substrate ubiquitination. One model suggests that each substrate-binding domain in a dimerized Skp1-F-box-Cul1 (SCF) CRL function separately to provide multiple geometries for substrate ubiquitination. Another model poses that two substrate-binding domains bind multiple sites on a single substrate. However, to our knowledge, this latter model has not been tested experimentally. Our understanding of CRL dimerization is further limited by the lack of crystallographic data for any dimerized CRL substrate adaptor.

In this chapter, a crystal structure encompassing both substrates binding, $\mathrm{Cul} 3$ binding and dimerization functionalities reveal how the dimeric SPOP can recruit a single substrate via multiple SBCs. As other researchers were unable to crystallize minimal constructs that contain the adjacent D domain and F-box domains, our results demonstrate the first substrate-bound adaptor dimer and provide a molecular foundation for understanding mechanisms underlying ubiquitination by BTB-Cul3s and other dimeric CRLs. 


\subsection{RESULTS}

\subsubsection{Structures of SPOP ${ }^{\text {MATH-BTB-3-box }}$}

In order to gain insights into how substrate, Cul3-binding and dimerization are encompassed in a single polypeptide, we determined the structure of a dimeric SPOP $^{\text {MATH-BTB-3-box }}$-Puc ${ }^{\text {SBC }}$ complex (Fig 5.1). In both structures, the MATH-SBCs, and the BTB-3box dimer, each resemble the structures of the individual domains. This includes the roughly 2-fold symmetry of the isolated BTB-3-box domain.

The most striking feature of the structures is the asymmetric arrangement of the two substrate-binding MATH domains, with respect to the BTB-3-box dimer. One MATH domain packs in the middle of the V-shaped groove created by the two protomers in the BTB domain. The other MATH domain is more distal, and extends away from its associated BTB domain, as if to extend the length of one side of the "V" (Fig 5.1).

\subsubsection{Flexibility within SPOP MATH-BTB-3-box $^{\text {MAty }}$}

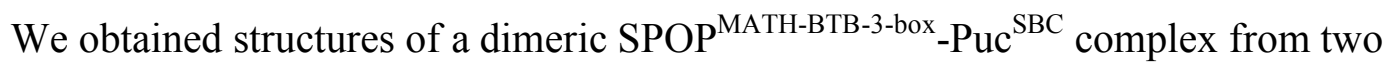
slightly different crystal forms (Fig 5.2A, B). Two features of the crystal structures suggest that the MATH and BTB domains are flexibly tethered. First, the linker between the MATH and BTB domains is not visible in the electron density in one crystal form (crystal 2). In the other, the backbone of one linker is visible, although the side-chains are not, allowing building only as polyalanine (crystal 1). Second, comparing the complexes in the two crystal forms reveal distinct positions of the MATH domains relative to each other. As shown in Fig 4.2C, the entire domain of MATH "B" in crystal form 1 (MATH_B1) is $\sim 3 \AA$ away from that in crystal form 2 (MATH_B2).

We performed two types of experiments to examine the linkage between the MATH and BTB domains in solution, using the two longest forms of SPOP that we could express: SPOP ${ }^{\mathrm{MATH}-\mathrm{BTB}-3-b o x}$ and SPOP ${ }^{\mathrm{N}-\mathrm{MATH}-\mathrm{BTB}-3-b o x}$. First, we performed limited proteolysis with endoproteinase Glu C, and observed that the inter-domain linker is susceptible to cleavage (Fig 5.3). Second, we performed Small Angle X-Ray Scattering (SAXS) analysis (performed by Dr. Michal Hammel, Lawrence Berkeley National Laboratory). In both cases, the data did not fit well with any single conformation. Instead, the data fitted well with a population of multiple conformations, although the presence of the $\mathrm{N}$ terminus appeared to restrict the overall compactness of the complex (Fig 5.4). Overall, the data are consistent with the notion of orientation variability between the BTB and MATH domains of SPOP. 


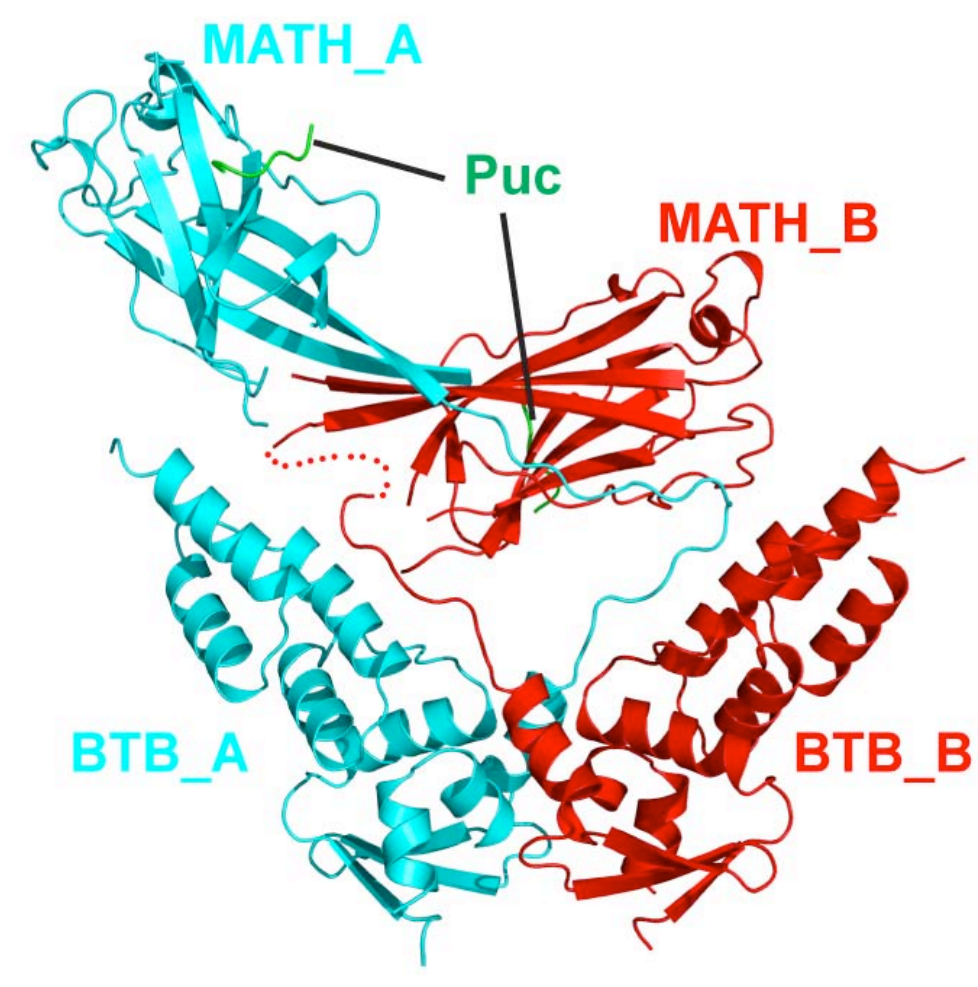

Figure 5.1. Overall architecture of SPOP ${ }^{\mathrm{MATH}-\mathrm{BTB}+}$ dimer. One molecule $(\mathrm{A})$ is colored in cyan and the other $(\mathrm{B})$ is in red. Each MATH domain binds one Puc ${ }^{\mathrm{SBC} 1}$ (green) peptide. Disordered regions not visible in the electron density are represented with dotted lines to show connectivity. 
A
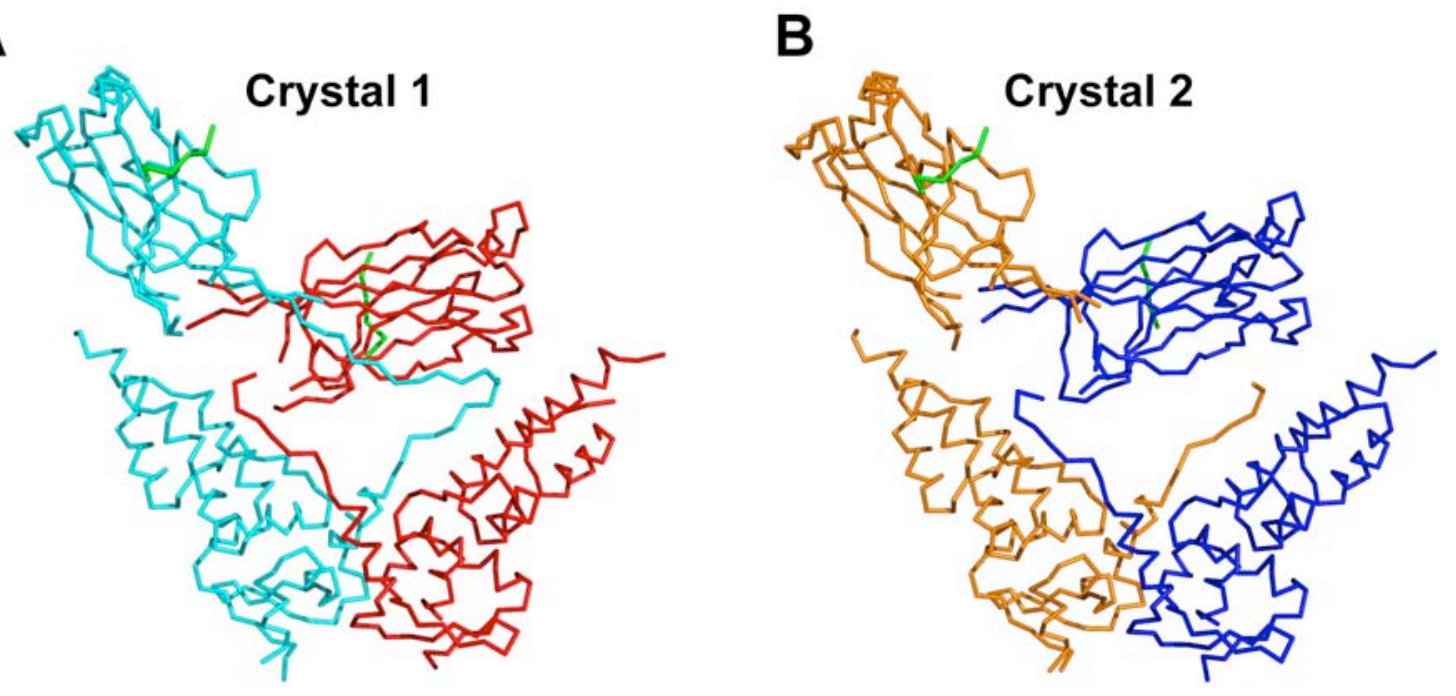

C

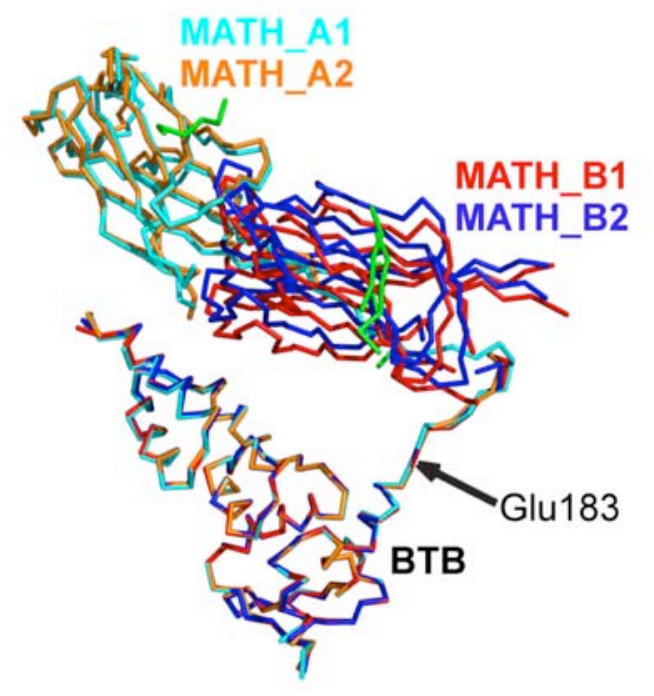

Figure 5.2. Superimposition over the BTB domains of SPOP ${ }^{\mathrm{MATH}-\mathrm{BTB}+}$ structures $^{-}$ determined from crystals with slightly different unit cells. MATH_A1 (cyan) and MATH_B1 (red) are from crystal form $1(\mathrm{a}=55.3 \AA, \mathrm{b}=106.8 \AA, \mathrm{c}=130.5 \AA)$, and correspond to the structure in (A). MATH_A2 (orange) and MATH_B2 (blue) are from crystal form $2(\mathrm{a}=63.5 \AA, \mathrm{b}=107.6 \AA, \mathrm{c}=1 \overline{3} 0.7 \AA)$, and correspond to the structure in $(\mathrm{B})$. Four molecules from two crystals are superposed over the BTB domain (C). 


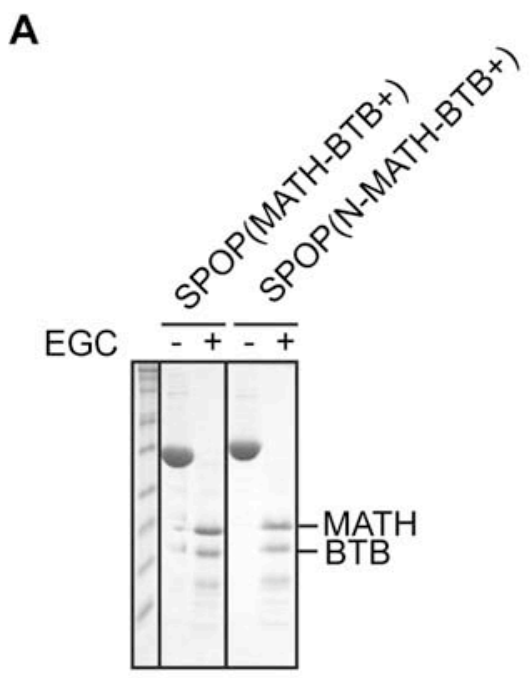

B Mass. ID of digestion product:

MATH BTB

SPOP (MATH-BTB+) 28-183 184-304/196-304

SPOP (N-MATH-BTB+) 21-183 184-304/196-304

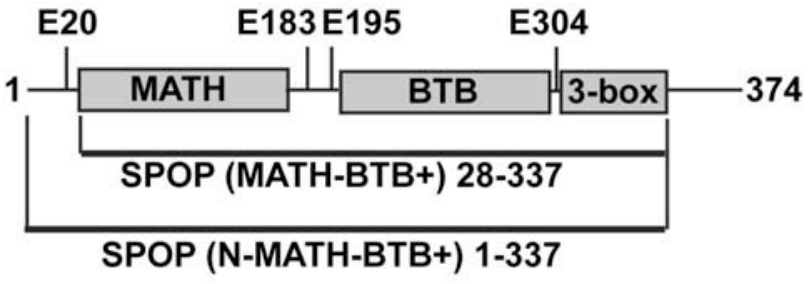

Figure 5.3. SPOP ${ }^{\mathrm{MATH}-\mathrm{BTB}+}$ and SPOP ${ }^{\mathrm{N}-\mathrm{MATH}-\mathrm{BTB}+}$ display similar susceptibilities to proteolytic digestion by Endoproteinase Glu-C. (A) Coommassie-stained $15 \%$ SDS-PAGE gels showing SPOP ${ }^{\mathrm{MATH}-\mathrm{BTB}+}$ and $\mathrm{SPOP}^{\mathrm{N}-\mathrm{MATH}-\mathrm{BTB}+}$ before and after 1-hour room temperature treatment with $5 \%$ Endoproteinase Glu-C. (B) Products from the proteolytic digestions identified by mass spectrometry (top). Endoproteinase Glu-C cleavage sites shown in a schematic view of primary structure is shown below. 

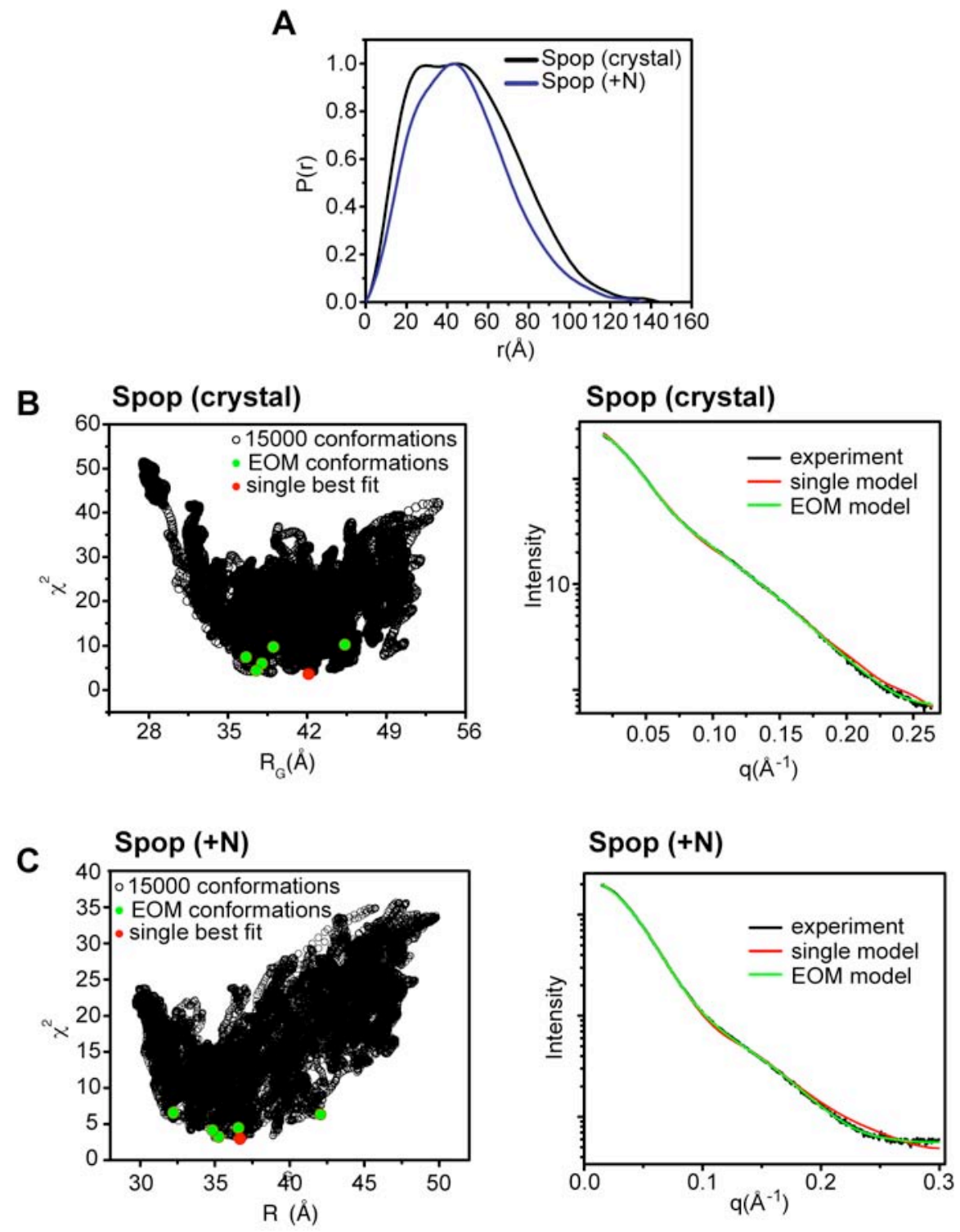

Figure 5.4. SPOP MATH and BTB domains are flexibly tethered in solution. Fig A is the Pair-distribution function $(\mathrm{P}(\mathrm{r}))$ for $\mathrm{SPOP}^{\mathrm{MATH}-\mathrm{BTB}+}$ (black, referred as SPOP crystal) and SPOP ${ }^{\mathrm{N}-\mathrm{MATH}-\mathrm{BTB}+}$ (blue, referred as SPOP $\left.+\mathrm{N}\right)$. The quality of fit $(\chi 2)$ for individual conformations sampled by MD and comparison of the experimental SAXS data (black) and theoretically calculated SAXS-scattering profiles for the single best fitting conformation (red) and EOM (Ensemble Optimization Method) conformations (green) were shown for $\mathrm{SPOP}^{\mathrm{MATH}-\mathrm{BTB}+}(\mathrm{B})$ and $\mathrm{SPOP}^{\mathrm{N}-\mathrm{MATH}-\mathrm{BTB}+}(\mathrm{C})$. 


\subsubsection{SPOP:Puc 2:1 Stoichiometry}

Given the dimeric nature of SPOP, and the orientational flexibility of the two substrate-binding domains, we examined the stoichiometry of binding to a substrate with multiple SBCs. To begin to address this question, we performed AUC analysis of a mixture of a 1:5 molar ratio of SPOP ${ }^{\text {MATH-BTB-3box }}$ and Puc ${ }^{1-390}$, which contains all three SBC motifs. Both velocity and equilibrium AUC analyses reveals two species with molecular weights of $\sim 110 \mathrm{kDa}$ and $\sim 40 \mathrm{kDa}$. These correspond to a 2SPOP:1Puc complex and free Puc (Fig 5.5). Thus, the SPOP dimer binds only one molecule of the substrate Puc in vitro, even in the presence of extra Puc.

\subsection{DISCUSSION}

The BTB domain of SPOP adopts a dimeric structure, which superimposes on a number of BTB domains whose structures have been previously solved. We found that the dimeric SPOP BTB domain can assemble with 2 molecules of Cul3, thereby generating a dimeric ubiquitin ligase containing two substrate-binding sites and two catalytic cores. Notably, Several other ubiquitin E3s have been shown to dimerize [41]. These include several CRLs, such as human $\mathrm{SCF}^{\mathrm{Fbw} 7}$ and its yeast counterpart $\mathrm{SCF}^{\mathrm{Cdc} 4}$ $[65,86]$.

To understand the function of SPOP, we generated models based on our multiple SPOP structures, and the available cullin crystal structures. Based on modeling of the dimeric SPOP-SBC complex with Cul3, the substrate-binding site is directed toward the catalytic core of the same cullin molecule to which the BTB domain is associated (Fig 5.6, Appendix A, Fig A.8). However, our crystallographic and SAXS data indicate that the MATH domain adopts multiple orientations relative to the BTB domain through the flexible linker between the two. This feature of $\mathrm{Cul}^{\mathrm{SPOP}}$ parallels that found with some other CRLs. The Cul4 adaptor DDB1 adopts multiple conformations [12, 13, 68]. Moreover, superposition of two molecules in one asymmetric unit of Cdc4 within the $\mathrm{SCF}^{\mathrm{Cdc} 4}$ crystal structure indicates different positions of the substrate binding domains, reminiscent of our findings with $\mathrm{SPOP}^{\mathrm{MATH}-\mathrm{BTB}}[48]$.

Recent structural studies indicate the ligation of NEDD8 to a conserved cullin Lys can serve as an additional source of conformational flexibility. Crystal structures revealed striking conformational differences between unmodified and neddylated versions of the Cul5 C-terminal in complex with Rbx1 [69]. Taken together with enzymological and biophysical analyses, the data indicate that NEDD8 causes a switch in CRL structure from a closed autoinhibited form, to a more dynamic active form [70, 71]. Thus, structural flexibility appears in many forms, for many CRLs.

The combination of dimerization and flexibility has many implications for substrate ubiquitination. First, the flexibility within SPOP may allow for SBCs in structurally diverse substrates to engage one or both MATH domains. Indeed, we found that Puc, which contains multiple functional SBCs, binds preferentially to SPOP in a 1:2 

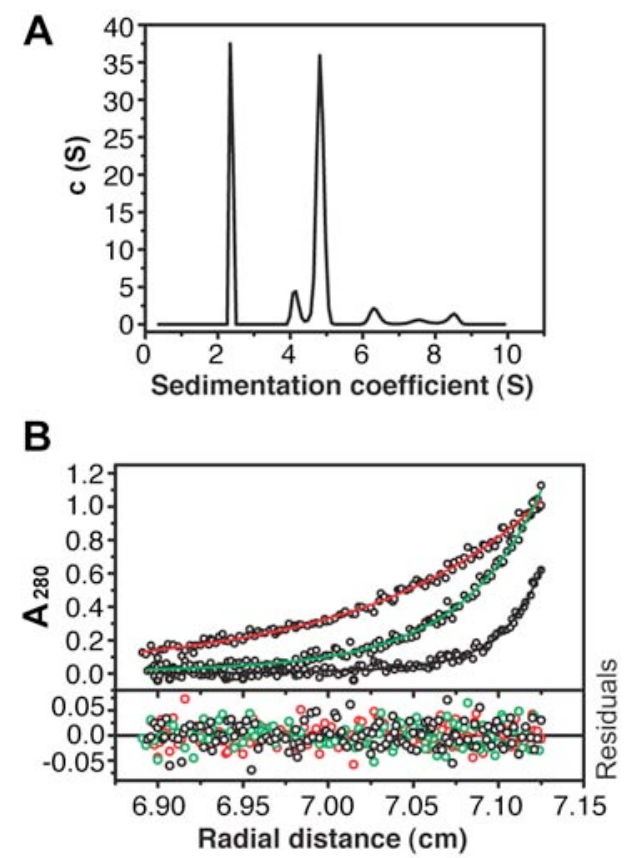

Figure 5.5. A 1:2 substrate complex with the SPOP-Cul3 ubiquitin ligase. (A) Velocity analytical ultra-centrifugation analysis of SPOP ${ }^{\mathrm{MATH}-\mathrm{BTB}+}$ and $\mathrm{Puc}^{1-390}$ mixed at a 1:2 molar ratio 1:2. The two peaks indicate molecular weights of $113 \mathrm{kDa}$ and $39 \mathrm{kDa}$, which correspond to a 1:2 Puc:SPOP ${ }^{\mathrm{MATH}-\mathrm{BTB}+}$ complex (calculated MW of 112,233 Da) and excess free Puc (calculated MW of 42,086 Da). (B) Equilibrium analytical ultracentrifugation analysis for a sample as in (A). Best-fit of all the data sets to a discrete species model with 2 species of molar masses of 112,233 and 42,086 kDa. 


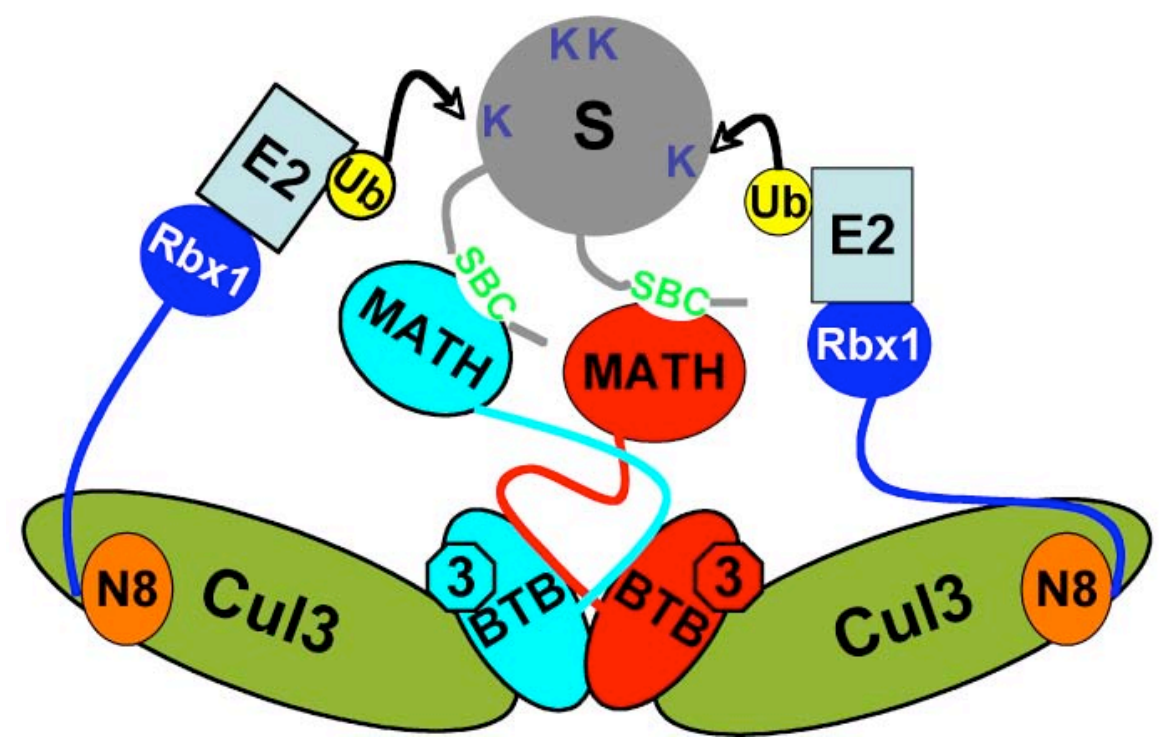

Figure 5.6. Model of the SPOP-Cul3 ubiquitin ligase. The substrate is shown in grey, with SBCs in flexible regions, and ubiquitin-acceptor lysines shown as K's. The two protomers of the dimeric SPOP complex are shown in cyan and red, with each bound near the $\mathrm{N}$ terminus of the elongated $\mathrm{Cul} 3$ (green) that has been activated with NEDD8 (orange) near the C-terminus. The E2-bound Rbx1 RING domains are flexibly tethered to the Cul3 C-terminal domains. The high degree of conformational flexibility may allow substrates with a range of SBC configurations to be polyubiquitinated at multiple sites. 
ratio, even when Puc is in excess. This suggests that a single Puc molecule engages both SBC binding sites in the SPOP dimer, although we cannot rule out the unlikely possibility that binding of the first Puc molecule to SPOP sterically blocks the association with the second Puc molecule (Fig 5.6). Notably, multiple E3-binding sites are also found in several other dimeric CRL substrates. For example, the Fbw7 substrate Cyclin E contains one optimal and one sub-optimal degron. Efficient turnover of a Cyclin E mutant in which the optimal degron is made sub-optimal requires Fbw7 dimerization [88]. Thus, the use of dimeric substrate binding domains may facilitate binding to substrates that have multiple degrons, or increase the efficiency with which substrates containing sub-optimal degron sequences can bind.

Interestingly, not all SPOP substrates have multiple SBCs (e.g. MacroH2A), suggesting that dual binding is not a universal feature of the $\mathrm{Cul} 3^{\mathrm{SPOP}} \mathrm{E} 3$. In such a case, it is conceivable that structural flexibility is coupled to ubiquitin conjugation and elongation, to allow the substrate and elongating ubiquitin chain to sample a greater variety of orientations. It is also possible that substrates harboring only a single SBC within their sequence may present multiple SBCs in the context of larger macromolecular assemblies, such as chromatin in the case of MacroH2A. Although the precise manifestations of dimerization are still under debate, it seems that the use of two flexibly oriented substrate binding sites, combined with a flexible cullin-RING core, would allow a single E3 to recognize numerous substrates with a range of conformational requirements for mediating their ubiquitination. 


\section{CHAPTER 6. DISCUSSION}

In this work, we studied the structure of SPOP, a human BTB protein that serves as a substrate adaptor for the largest CRL subfamily, the Cul3-based ubiquitin ligases. We identified the common SBC motif on four SPOP substrates and studied the interactions between $\mathrm{SPOP}^{\mathrm{MATH}}$ and the SBC sequences on the substrates both in vitro and in vivo. We structurally characterized a novel Cul3-binding component, the 3-box, in SPOP and Gigaxonin and predicted its presence in a subset of BTB proteins. Finally, we characterized the flexibility of SPOP. Results from our study have many implications for understanding the specific biological roles of $\mathrm{Cul}^{\mathrm{SPOP}}$ in each SPOP mediated pathway and revealing specific aspects of Cul3-based ubiquitin ligases to build the general structural architectures of CRLs.

\subsection{IMPLICATIONS FOR UBIQUITIN-MEDIATED REGULATION OF THE HEDGEHOG PATHWAY}

\subsubsection{What Is the Role of the HIB-SBC Interaction in the Drosophila Hedgehog Pathway?}

The Hedgehog (Hh) signaling pathway governs cell growth and plays crucial roles in animal development. In Drosophila, Hh binds its membrane receptor Patched (Ptc), which negatively regulates another transmembrane protein Smoothened (Smo). In the absence of Hh, Ptc prevents Smo translocation to the cell surface; the key transcriptional regulator Cubitus interruptus (Ci) forms a complex with the atypical kinesin protein Costal 2 (Cos2), the Ser/Thr kinase Fused ( Fu) and the suppressor of Fused (Sufu) and is sequenstered in the cytoplasm as the inactive form, $\mathrm{Ci}^{155}$. Part of $\mathrm{Ci}^{155}$ undergoes a sequential phosphorylation by protein kinase A (PKA), glycogen synthase kinase (GSK) and casein kinase I (CKI) to generate phosphodegrons that can be recognized by the Cul1 ${ }^{\text {Slimb }}$ ubiquitin ligase $[77,105,126]$. Ubiquitination of $\mathrm{Ci}^{155}$ by $\mathrm{Cul} 1^{\text {Slimb/ } / \text {-TrCP }}$ leads to the proteasome-dependent cleavage of $\mathrm{Ci}^{155}$ into $\mathrm{Ci}^{75} \cdot \mathrm{Ci}^{75}$ is further translocated into the nucleus and functions as a transcriptional repressor. In the presence of a high level of Hh, Ptc can no longer inhibit Smo, which then accumulates on the cell surface. As Cos2 and Fu are recruited to Smo, they release $\mathrm{Ci}^{155}$ to be translocated into the nucleus and function as a transcriptional activator (reviewed in [159, 160]). $\mathrm{Ci}^{155}$ activation induces $\mathrm{Hh}$-induced MATH and BTB domain containing protein (HIB, the Drosophila ortholog of SPOP), which in turn functions in a negative feedback loop to inhibit $\mathrm{Ci}^{155}$ by mediating $\mathrm{Cul}^{\mathrm{HIB}}$-dependent ubiquitination and degradation of $\mathrm{Ci}^{155}[4,141]$.

Dual ubiquitination pathways regulate $\mathrm{Ci}$, but what determines whether $\mathrm{Ci}$ undergoes $\mathrm{Cul1}{ }^{\text {Slimb }}$-dependent proteolysis or $\mathrm{Cul}^{\mathrm{HIB}}{ }^{\mathrm{HI}}$-dependent degradation? Previous studies have suggested that two factors account for the switch from Cul $1^{\text {Slimb }}$ - to

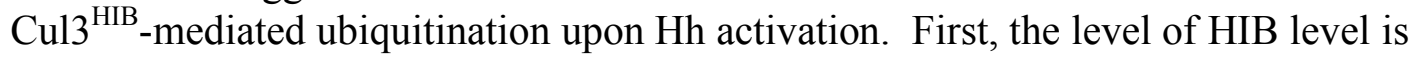


regulated by $\mathrm{Hh}$ signaling; therefore, the level of $\mathrm{Cul}^{\mathrm{HIB}}$ is higher in the presence of $\mathrm{Hh}$, which allows $\mathrm{Cul} 3^{\mathrm{HIB}}$-dependent ubiquitination to dominate [4]. Second, localization might play a role in Ci regulation. HIB is localized in the nucleus, and the Sufu-Fu-Cos2 bound $\mathrm{Ci}^{155}$ stays in the cytoplasm. In the presence of $\mathrm{Hh}, \mathrm{Ci}^{155}$ is released from the $\mathrm{Fu}-\mathrm{Cos} 2$ complex and translocated to the nucleus, where it is accessible to $\mathrm{Cul} 3^{\mathrm{HIB}}[161$, $162]$.

Our identification of the $\mathrm{SBC}$ motif on $\mathrm{Ci}^{155}$ provides a third factor that might account for the selective ubiquitination by $\mathrm{Cul}^{\mathrm{HIB}}$ in the presence of Hh. The SBC motif, $\phi-\varsigma-\mathrm{S}-\mathrm{S} / \mathrm{T}-\mathrm{S} / \mathrm{T}$, contains several serines and threonines (Ser/Thr). Because CRL substrates usually contain phosphorylated Ser/Thr or Tyr, we wondered whether the phosphorylation of an SBC can affect HIB/SPOP binding. In Chapter 3, we substituted each Ser/Thr on two SBC peptides and examined the interactions between SPOP ${ }^{\mathrm{MATH}}$ and the phosphorylated peptides. SPOP ${ }^{\mathrm{MATH}}$-peptide interaction was abolished by the phosphorylation of $\mathrm{P} 3$ or $\mathrm{P} 4 \mathrm{Ser} / \mathrm{Thr}$ and was significantly attenuated by the phosphorylation of $\mathrm{P} 5 \mathrm{Ser} / \mathrm{Thr}$. This finding raises the possibility that phosphorylation negatively regulates $\mathrm{Cul} 3^{\mathrm{HIB}}$-dependent degradation of $\mathrm{Ci}^{155}$. If so, the potential phosphorylation of Ci SBC sites may abolish HIB recognition, thereby turning off $\mathrm{Cul}^{\mathrm{HIB}}$ mediated ubiquitination in the absence of $\mathrm{Hh}$.

To test this hypothesis, an answer is needed for the following question: are the $\mathrm{Ci}$ SBC sites phosphorylated in vivo? Previous studies have shown that $\mathrm{Ci}$ is indeed phosphorylated by PKA, GSK and CKI for Cul1 ${ }^{\text {Slimb }}$-dependent ubiquitination in vivo [163-167]. However, the phosphorylation sites reported by those groups differ from the SBC sites that we identified. Given that PKA, GSK and CKI target Ser/Thr and the SBC sites need to be exposed on the surface of the protein to be recognized by HIB, it is rational to predict that Ser/Thr-rich SBC sites are also phosphorylated by these kinases. In addition, the $\mathrm{Fu}$ substrates have not been characterized. $\mathrm{Cos} 2, \mathrm{Ci}^{155}$ and $\mathrm{Fu}$ form a complex in the absence of $\mathrm{Hh}$; thus, $\mathrm{Ci}$ SBC sites may be the targets of Fu. If the SBC sites are most likely to be phosphorylated, then why was the phosphorylation of SBC not previously identified in Smelkinson's paper [168], which first identified phosphorylated Slimb binding sites? This may be explained by the limitations of their experimental procedures. Smelkinson's group used different kinases to phosphorylate a truncated form of Ci that lacked both SBC sites, and they looked for the Slimb-binding protein by immunoprecipitation. To determine whether the Ci SBC sites are phosphorylated in vivo, it may be necessary to use alternative approaches, such as mass spectrometry characterization of immunoprecipitated cellular $\mathrm{Ci}$. It is also possible that the SBCs are only phosphorylated under particular, currently unknown conditions.

The next question that must be answered is how does the phosphorylation of the SBC sites regulate the fate of $\mathrm{Ci}$ ? In the absence of $\mathrm{Hh}$, the phosphorylation of the SBC sites may protect both $\mathrm{Ci}^{155}$ and $\mathrm{Ci}^{75}$ from $\mathrm{Cul}^{\mathrm{HIB}}$-mediated degradation. In the presence of $\mathrm{Hh}$, HIB recognizes $\mathrm{Ci}^{155}$, but not $\mathrm{Ci}^{75}$. If $\mathrm{Ci}^{155}$ is phosphorylated in the absence of $\mathrm{Hh}$, this process will require de-phosphorylation in the presence of $\mathrm{Hh}$. The Hh pathway may include an as yet unidentified phosphatase, but it is more likely that only $\mathrm{Ci}^{75}$ is protected from degradation by phosphorylation, and $\mathrm{Ci}^{155}$ uses an alternative mechanism 
to avoid HIB binding in the absence of Hh. Sufu and HIB competitively bind the same region on $\mathrm{Ci}$ [4]; therefore, Sufu binding may inhibit phosphorylation and HIB binding to $\mathrm{Ci}^{155}$.

In addition, regardless of the regulation by phosphorylation, our two-sites binding model (as described in Chapter 5) helps explain the preferred ubiquitination of $\mathrm{Ci}^{155}$ over that of $\mathrm{Ci}^{75}$. $\mathrm{Ci}^{155}$ contains two SBC sites and the $\mathrm{N}$-terminal fragment $\mathrm{Ci}^{75}$ contains only one SBC site. Our model predicts that in the presence of both $\mathrm{Ci}^{155}$ and $\mathrm{Ci}^{75}$, HIB binds $\mathrm{Ci}^{155}$ with higher affinity, and $\mathrm{Cul} 3^{\mathrm{HIB}}$ preferentially recognizes $\mathrm{Ci}^{155}$ for ubiquitination dependent degradation.

Here we propose the following model based on our findings of the Ci SBC sites. In the absence of $\mathrm{Hh}$, part of $\mathrm{Ci}^{155}$ is sequestered in Sufu-Cos2 in the cytoplasm, and phosphorylation of SBC sites is inhibited by Sufu binding. Another part of $\mathrm{Ci}^{155}$ binds Fu-Cos2 and is extensively phosphorylated by PKA, GSK, CKI and Fu. Phosphorylated $\mathrm{Ci}^{155}$ is processed to the transcriptional repressor $\mathrm{Ci}^{75}$, where only one SBC site remains,

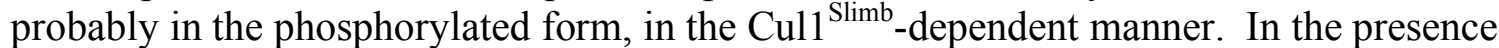
of $\mathrm{Hh}, \mathrm{Ci}^{155}$ is released from Sufu-Cos 2 and functions as a transcriptional activator in the nucleus, where it promotes HIB expression. HIB is then assembled into the $\mathrm{Cul} 3$ ubiquitin ligase to ubiquitinate $\mathrm{Ci}^{155}$ and degrade it in a proteasome-dependent manner. It will be interesting to see in the future how indeed $\mathrm{Ci}$ degradation is distributed between the different E3s.

\subsubsection{What Is the Role of the SPOP-SBC Interaction in the Human Hedgehog Pathway?}

The human Hedgehog pathway involves more components and is much more complicated than the Drosophila Hedgehog pathway. The first question to be asked: is the E3 activity of SPOP conserved from Drosophila to mammals? Several previous findings support a conserved role of SPOP in humans: (1) The Hedgehog pathway, although evolutionarily diverse, has a similar framework and key components in Drosophila and human; (2) The expression of human SPOP can reverse the phenotype caused by the deletion of HIB [125]; (3) The HIB substrate Ci has its functional parallels in humans that are known as Gli1, Gli2 and Gli3; (4) HIB mediates the degradation of human Gli2 and Gli3 when these proteins are transfected and expressed in insect cells [4].

The next question to be asked is: does $\mathrm{Cul} 3^{\mathrm{SPOP}}$ mediate the ubiquitination and degradation of Gli proteins? Given that SPOP and HIB sequences are highly conserved and human SPOP ${ }^{\mathrm{MATH}}$ crystallized in complex with Drosophila Ci peptide, we propose that SPOP binds Gli proteins through the SBC motif. Our analysis of the sequences of Gli1, Gli2 and Gli3 revealed that not all Gli proteins include an SBC motif. Gli1 contains no SBC sequences; Gli2 contains one SBC sequence, "ASSTS", corresponding to residues 630-634, and Gli3 contains one SBC sequence, "ASSTT", corresponding to residues 35-39 and two SBC variants "PSTSS" corresponding to residues 75-79 and 
"VSSTG" corresponding to residues 423-427. Thus, Gli2 and Gli3, but not Gli1, are most likely the substrates of $\mathrm{Cu} 13^{\mathrm{SPOP}}$. The previously mentioned finding, that HIB mediates the degradation of Gli2 and Gli3 transfected and expressed in insect cells, is consistent with this notion.

Notably, Gli2 and Gli3 are the Gli proteins most similar to $\mathrm{Ci}$, because they can function as either a transcriptional activator in full length or a transcriptional repressor in a shorter form. In contrast, Gli1 always functions as a transcriptional activator. Given the differences between Gli1, Gli2 and Gli3, the selective ubiquitination and degradation of Gli2 and Gli3 by Cul3 ${ }^{\mathrm{SPOP}}$ may help explain the discrepancies between the Drosophila and mammalian Hedgehog pathways. For example, Sufu-null mice are embryonic lethal but Sufu-null flies are viable and fertile. Sufu regulates $\mathrm{Ci} /$ Gli nuclear localization and $\mathrm{Ci} /$ Gli-activator activity, thereby negatively regulating Hh signaling $[161,167]$. In Drosophila, $\mathrm{Cul}^{\mathrm{HIB}}$, another negative regulator of $\mathrm{Hh}$ signaling, may compensate for the loss of Sufu. But in mammals, $\mathrm{Cul} 3^{\mathrm{HIB}}$ only partly inhibits Hh signaling by targeting Gli2 and Gli3 but not Gli1; other negative regulators, such as Sufu, may be required for Gli1 regulation. This explains why Sufu is more essential in mammals than in flies.

\subsection{IMPLICATIONS FOR APOPTOSIS}

HIB/SPOP is linked to apoptosis in both Drosophila and humans. In Drosophila, HIB regulates Jun N-terminal kinase (JNK) signaling by targeting the mitogen-activated protein kinase (MAPK) phosphatase Puc, which in turn promotes cell death $[58,169]$. This is supported by the finding that in Drosophila the deletion of HIB rescues the small-eye phenotype caused by tumor necrosis factor (TNF) triggered cell death through JNK signaling [58]. In mammals, SPOP mediates the ubiquitination of the apoptosis regulator Daxx, which was originally identified as a protein associated with the death domain of the Fas receptor and later was found to promote Fas-mediated apoptosis via activation of JNK [170-172]. Recently, Daxx was identified as a key regulator of Mdm2. Daxx inhibits auto-ubiquitination of Mdm2 through a de-ubiquitinating enzyme (DUB) known as herpesvirus-associated ubiquitin-specific protease (HAUSP) [173]. Our structural study of SPOP ${ }^{\mathrm{MATH}}$-SBC complexes and identification of the SBC motif have resulted in several hypotheses about the role of SPOP in apoptosis.

First, we noticed that there are several MATH/TRAF domain-containing proteins (i.e., TRAF2, TRAF3 and TRAF6) involved in TNF family receptor signaling. The established role of these TRAF domains is to inhibit NF-KB induction $[174,175]$. Structures of the TRAF domains of different TRAF proteins have revealed conservation at the substrate binding sites [147-151]. In contrast, comparisons of SPOP ${ }^{\mathrm{MATH}}$ and TRAF2/3/6 ${ }^{\mathrm{TRAF}}$ structures have revealed conserved overall architectures but significantly different amino acid compositions at the substrate-binding sites, indicating distinct substrate binding and biological functions of SPOP ${ }^{\mathrm{MATH}}$ and classical TRAFs [119, 142]. This structure-based prediction is further supported by the experiment showing that $\mathrm{SPOP}^{\mathrm{MATH}}$ cannot inhibit NF-кB induction [142]. 
Second, we noticed that HAUSP contains the MATH domain. HAUSP is a de-ubiquitinating enzyme that modifies ubiquitination levels of p53 and Mdm2, thereby, regulating $\mathrm{p} 53$-dependent apoptosis $[144,145,176]$. Structure superimposition of HAUSP $^{\mathrm{MATH}}$ and SPOP ${ }^{\mathrm{MATH}}$ revealed nearly identical substrate-binding sites. In addition, like the SPOP ${ }^{\mathrm{MATH}}$-SBC interaction, $\mathrm{HAUSP}^{\mathrm{MATH}}$-substrate binding requires a conserved Ser on Mdm2 or p53 peptides [145, 146]. Therefore, we propose that SPOP and HAUSP share substrates by binding the same substrate sequences via their MATH domains.

This hypothesis is supported by our own study (1) and clues from previous studies (2-3): (1) To determine whether SPOP ${ }^{\mathrm{MATH}}$ and HAUSP ${ }^{\mathrm{MATH}}$ bind the same substrates, we tested the binding of different human substrate peptides to SPOP ${ }^{\mathrm{MATH}}$ and HAUSP $^{\mathrm{MATH}}$ by BIACORE. The HAUSP substrate peptide containing Mdm2 residues "ELQEEKPSSSHLVSR," which is most similar to the SBC motif among all HAUSP substrate peptides, bound both SPOP ${ }^{\mathrm{MATH}}$ and HAUSP ${ }^{\mathrm{MATH}}$ with similar affinities, indicating that Mdm2 might also be a substrate of SPOP (Appendix A, Fig A.4); (2) Both SPOP and HAUSP are localized in the nucleus, as are the potential common substrates Mdm2 and Daxx [16, 120, 177]; (3) Daxx is the common binding partner of SPOP and HAUSP. Although we were unable to detect any interactions between HAUSP ${ }^{\mathrm{MATH}}$ and $\operatorname{Daxx}^{\mathrm{SBC}}$ due to the poor solubility of the Daxx SBC peptide in vitro, it has been reported by Tang et.al [173] that Daxx associates with HAUSP and Mdm2 to form a ternary complex in vivo and Daxx enhances the DUB activity of HAUSP towards Mdm2. In their model, a Daxx-HAUSP-Mdm2 complex stabilizes Mdm2 and leads to Mdm2-dependent p53 ubiquitination and degradation under nonstress conditions. After DNA damage, HAUSP and Daxx dissociate from Mdm2, which triggers autoubiquitination and degradation of $\mathrm{Mdm} 2$ and p53-dependent apoptosis. In another paper, Kwon et.al [116] demonstrated that SPOP recruits Daxx to Cul3 for ubiquitination and subsequent degradation by the proteasome, and Daxx degradation triggers apoptosis. Results from these two papers suggest that SPOP forms a ternary complex with Daxx and Mdm2 and elicits a function that is opposite to the one elicited by HAUSP. Therefore, SPOP and HAUSP may function as a pair consisting of E3 ubiquitin ligase and DUB de-ubiquitinating enzyme to regulate the ubiquitination of the same substrates.

Why would a cell develop two enzymes with opposite functions toward the same substrate? Because this allows the cells to dynamically regulate substrates. Many examples of this type of regulation have been shown in studies of kinases and phosphatases. For example, kinase Wee1 and phosphatase CDC25 cooperatively regulate Cdc2-Cyclin B during cell cycle for precise transition into mytosis. Notably, Wee1 and CDC25 themselves are regulated by post-translational modifications as reviewed by Perry et al [178]. There are also a few opposing pairs of ligases and deubiquitinating enzymes. For example, the deubiquitinating enzyme Ubp2 modulates K63-polyubiquitination of Csr2 and Ecm21, which are the substrates of ubiquitin ligase Rsp5 [179, 180]; the products of proteasome-associated ligase Hul5 are dissembled by the proteasome-bound deubiquitinating enzyme Ubp6 [181]; the NF-кB inhibitor A20 compasses a deubiquitinating domain at the $\mathrm{N}$ terminus and a ligase domain at the $\mathrm{C}$ terminus to remove K63-linked ubiquitin chains and build K48-linked ubiquitin chains on 
the same substrate [9]. Dynamic ubiquitination is important for remodeling the length of the ubiquitin chain (in case of Hul5 and Ubp6) and for determining the type of ubiquitin chain (in case of A20) that regulates substrate for degradation.

Unlike the regulation of kinase and phosphatase pairs, that of the E3 and DUB pair is not well characterized. Given Hul5 and Ubp6 are situated in close proximity on the proteasome [181], and a single polypeptide A20 harbors both E3 and DUB function, SPOP and HAUSP are localized in the nucleus; thus, different localizations may not explain the regulation of dynamic ubiquitination. In addition, if SPOP and HAUSP bind the same substrate sequences containing the SBC motif, post-translational modifications of the SBC motif may not increase the preference of substrate towards E3 or DUB. Because the level of SPOP can be induced, circumstances such as DNA damage may stimulate SPOP expression. Alternatively, SPOP and HAUSP may function cocurrently, like the Hul5 and Ubp6 pair or A20, thereby remodeling ubiquitin chains to dynamically balance Mdm2 and P53 levels. In this scenario, SPOP and HAUSP might compete for substrate binding, because they bind the same sequences. Protein levels and affinities between each MATH and the substrate may determine the outcome of the ubiquitination level of the substrate.

Interestingly, the structure of the larger HAUSP fragment encompassing the MATH domain and the catalytic core (2F1Z.pdb) indicated the HAUSP MATH domain is flexibly linked to the catalytic core [146]. Similar to SPOP ${ }^{\mathrm{MATH}}, \mathrm{HAUSP}^{\mathrm{MATH}}$ is linked to the catalytic domain through an unstructured peptide, which has high $\mathrm{B}$ factors in the structure. The crystal structure contains two HAUSP molecules in the asymmetric unit, molecule A and molecule B. When the catalytic domain of molecule A was superimposed onto that of molecule B, the orientations of the MATH domain were dramatically different than those we observed in SPOP structures. Thus, the flexibility of the substrate-binding domain may be a common feature of $\mathrm{Cul}^{\mathrm{SPOP}}$ and HAUSP. Moreover, HAUSP dimerizes in vivo [182]. Thus, dimerization may be another common feature of $\mathrm{Cul}^{\mathrm{SPOP}}$ and HAUSP. Considering these common features, we propose that dimerization and flexibility play similar roles in the processes of ubiquitination and deubiquitination. Like ubiquitination, deubiquitination requires that the substrate iso-peptide bond on Lys within a reasonable distance from the catalytic core. Given that HAUSP substrates vary in size and ubiquitin chains vary in length, the adjustable distance between the substrate-binding domain and the catalytic core introduced by dimerization and flexibility of HAUSP provides a potential solution.

Finally, we wondered whether $\mathrm{Cul}^{\mathrm{HIB}}$ mediated degradation of Puc in Drosophila is conserved in mammals. To answer this question, we looked for a Puc ortholog that could be ubiquitinated by $\mathrm{Cul} 3^{\mathrm{SPOP}}$. The human TNF pathway contains more than 20 phosphatases, each of which could be the Puc ortholog. With such a large number of candidates in vivo, identification of the Puc ortholog is a complex task. Identification of the SBC motif may help identify new substrates. In fact, we have screened the sequences of 20 Puc ortholog candidates and found seven phosphatases that contain the SBC motif. These phosphatases are more likely to be substrates of SPOP. Future studies need to focus on these phosphatases and examine their relation with SPOP. 


\subsection{SPOP: A YIN-YANG LIGASE}

Since the first crystal structure of the CRL component, EloB-EloC-VHL, was determined in 1999 [183], a growing number of efforts have been made to determine the structure and build the common architectures of CRLs. Several models of CRLs, including the rigid model, the flexible model and the dimerized model, have been proposed. To better understand these models, we propose to address the question: what is the role of rigidity and flexibility in CRL E3 activity?

Previous studies have shown that rigidity is important for CRL E3 activity. First, Zheng et al [45] made a Cull mutant in which the interfaces of the N-terminal domain and the C-terminal domain were disrupted by five hydrophobic-to-hydrophilic residue mutations, and the two domains were linked by a flexible linker containing "KGTREGKGSPEG". They found that the flexibly linked Cul1 mutant was not able to ubiquitinate the substrate $\mathrm{p} 27^{\mathrm{Kip} 1}$ in vitro. This result suggests that the rigidity of the Cull scaffold is important for E3 activity [45]. The rigidity of cullin was further confirmed by the crystal structure of Cul4 [13]. In the DDB1-Cul4-Rbx1 structure, Cul4 resembles the arc-shape of Cul1, despite the different space groups of the two structures. Second, Cand1 regulates the CRL assembly in vivo by binding cullins $[83,84]$. The structure of the ternary complex of Cand1, Cul1, and Rbx1 shows that Cand1 tightly wraps around Cul1, which maintains the same conformation as that observed in the free Cul1 structure [85]. Thus, cullin needs to be rigid for the docking of Cand1. Finally, although no consensus sequences for ubiquitin-accepting lysines have been identified, the ubiquitination of substrates occurs only at certain lysines. For example, $\mathrm{p} 27^{\mathrm{Kip} 1}$ and IкB $\alpha$ are both ubiquitinated at specific lysines close to their F-box protein-binding sites [184, 185]. The protein $\beta$-catenin is preferentially ubiquitinated at lysines 9 to 13 residues away from the $\beta$-TrCP-binding site [65]. The spatial and conformational constraints imposed by CRLs may determine the selection of lysines for ubiquitination.

Flexibility is also required for CRL E3 activity. First, in the rigid model of CRL bound to both substrate and E2, there is a gap of approximately $50 \AA$ between the E2 catalytic cysteine and the substrate peptide [45]. This gap needs to be closed for the ubiquitination reaction to occur. Second, many CRLs have been characterized with E3 activity towards different substrates, and most CRLs catalyze K48-linked polyubiquitin chains on substrate for proteasome-dependent degradation [38, 186, 187]. To adapt substrates of different size and with varying lengths of attached polyubiquitin chains, the CRL machinery needs to be flexible. Finally, in an active form of CRL, the ubiquitin-like protein NEDD8 covalently modifies the $\mathrm{C}$ terminus of cullin. Recent studies have shown a dramatic conformational change of CRL upon NEDD8 modification [41, 69-71]. Rbx1 is flexibly tethered to the NEDD8 modified C-terminal domain of cullin. Linker deletions or proline substitutions were introduced to hinder Rbx1 RING orientational flexibility. These mutants demonstrated decreased E3 activity [69].

To summarize, both rigidity and flexibility are required for CRL activity. The scaffold cullin is the main contributor to rigidity; and neddylation is the main contributor 
to flexibility. These finding leads to the continuing debate: do substrate adaptors contribute to the rigidity or flexibility of CRLs? On one hand, the crystal structure of Cul1 adaptor Skp1-Skp2 shows a rigid coupling between the Skp2 substrate-binding domain and Skp1 [46]; and several WD40 F-box protein structures have linkers composed of four to six tightly packed $\alpha$ helices between the substrate-binding domain and Skp1-F-box, indicating limited flexibility of these adaptors [44, 48, 65]. On the other hand, the Cul4 adaptor DDB1 in the DDB1-Cul4-Rbx1 complex structure adopts a new domain arrangement that is dramatically different from prior DDB1 alone or DDB1-SV5-V structures in the relative orientation between the substrate-binding domain and Cul4-binding domain. Our study of SPOP ${ }^{\mathrm{MATH}-\mathrm{BTB}}$ also revealed the multi-conformational nature of the substrate binding MATH domain with a flexible linker between it and the BTB domains.

The rigidity and flexibility of each substrate adaptor may not be absolute. The rigid adaptors may have certain flexibility. For example, the crystal structure of the rigid substrate adaptor Cdc4 contains two molecules in the asymmetric unit [48]. Superimposition revealed that the entire substrate-binding WD40 domains of the two molecules are about $3 \AA$ apart, where the Skp1-F-box regions superimpose well. This indicates some but limited flexibility within these "rigid" adaptors. Conversely, the flexible adaptors may have additional conformation restraints. We performed SAXS analysis with two forms of SPOP protein, SPOP ${ }^{\mathrm{MATH}-\mathrm{BTB}}$ and SPOP ${ }^{\mathrm{N}-\mathrm{MATH}-\mathrm{BTB}}$. The latter has 27 more amino acid residues at the $\mathrm{N}$ terminus and is more similar to wild type SPOP than is the former. Although both proteins demonstrated multi-conformational property in SAXS analysis, SPOP ${ }^{\mathrm{N}-\mathrm{MATH}-\mathrm{BTB}}$ displays a more collapsed character than does SPOP ${ }^{\mathrm{MATH}-\mathrm{BTB}}$, indicating that some restraints of conformation were introduced by the 27 N-terminal residues (Fig 5.4). Thus, we conclude that substrate adaptors contribute to both the rigidity and flexibility of CRLs.

SPOP is the best example of how rigidity and flexibility are coupled into a single adaptor. Flexibility and conformational restraints comprise the yin and yang of SPOP. On one hand, the SPOP MATH domain is flexibly tethered to the BTB domain. Given that the size of SPOP substrates ranges from $45 \mathrm{kDa}$ to $155 \mathrm{kD}$, the flexibly linked MATH domain may allow different substrates to be recruited to Cul3. In addition, in our two-sites binding model (as discussed in Chapter 5), the distance between the two SBC sites varies across substrates. The flexibility of SPOP allows the two MATH domains in the dimer to adjust their distance to bind two SBC sites on a single substrate. On the other hand, conformational restrains are introduced to SPOP by the length of the linker and the $27 \mathrm{~N}$-terminal amino acids through unknown mechanisms. Although the particular lysines that are ubiquitinated on SPOP substrates have not been identified yet, like other CRLs, SPOP probably modifies selective lysines. Therefore, restrained SPOP conformation may play a role in selective ubiquitination and assist in substrate binding. In the two-sites binding model, if the SBC sequences bind SPOP MATH domains in an orderly manner, then restrained MATH conformation may present the substrate better to be recognized by the second MATH domain. Overall, SPOP balances flexibility and conformational restraints to present substrates within the accessible range of Rbx1-E2 to perform ubiquitination at selective sites. In this yin-yang balance, the linker between the 
MATH and BTB domains is essential. It not only introduces flexibility into SPOP but also restrains the distance between MATH and BTB. Sequence alignment of SPOP proteins from different species revealed that the length of the linker was conserved across species; this finding emphasizes the importance of that linker. However, sequence alignment of different MATH-BTB proteins showed various linker lengths. So far, only two MATH-BTB proteins, HIB/SPOP and Mel-26, have been characterized with Cul3-based E3 activity. It is possible that other MATH-BTB proteins with different linkers do not function as substrate adaptors or the slightly different linkers still managed to keep the substrates within the range accessible to E2. To better understand the balance between flexibility and conformational restrains of SPOP, future studies needs to focus on the role of the linker.

\subsection{CRL DIMERIZATION}

Substrate adaptor mediated dimerization is essential in vivo in many Cull and Cul3-based CRLs. However, the molecular mechanisms that control this process are still not understood. Currently, there are two hypotheses about the dimerization of CRLs. The first suggests that dimerization accommodates multiple geometries for substrate ubiquitination by providing two orientations of substrate-binding domains [86]. This theory is supported by the structure study of the Cdc4 D-domain and the SAXS analysis of the F-box protein Cdc4 dimer done by Tang's group [86]. Their SAXS solution-based model displays a dimeric Cdc4 with two parallel substrate-binding WD40 domains pointing to the same direction. The dimeric Cdc4-Cul1-Rbx1-E2 model based on their SAXS data and crystal structures of Skp1-F-box-Cul1-Rbx1 [45] and c-Cbl-UbcH7 [37] displays different distances between one E2 catalytic cysteine and two substrate peptides, where the intramonomer distance is $64 \AA$ and the intermonomer distance is $102 \AA$. In addition, the distance between the two substrate-binding sites on $\mathrm{Cdc} 4$, where substrate Sic1 degrons bind, is $65 \AA$. Given that the mean distance between the Sic1 degrons is about $30 \AA$, the authors proposed that Sic1 probably cannot simultaneously engage both degron-binding sites. Therefore, they concluded that dimerization contributes to the different geometries.

The second hypothesis about dimerization of CRLs suggests that the existence of two substrate-binding domains increase the binding to substrates when substrate has multiple binding sites [65]. Although there is no direct evidence for this hypothesis, studies of Sic1 and cyclin E, as well as our own work, support this hypothesis. Sic1 and cyclin E have multiple degrons, and among these, some have high affinities to adaptors, and others have suboptimal affinities. Experiments have demonstrated that multiple degrons are required for the ubiquitination of Sic1 and cyclin $\mathrm{E}$ in vivo $[86,88,188]$. In Chapter 2, we also identified three SBC sites on Puc, one optimal binding site (SBC1) and two sub-optimal binding sites (SBC2 and SBC3). In our ubiquitination assay, substitutions of either one of the sub-optimal sites (SBC2 and SBC3) with non-SBC sequences barely affected the ubiquitination of Puc. In contrast, when both suboptimal SBC sites were mutated, the ubiquitination of Puc was greatly reduced. Thus, the ubiquitination of Puc is normal when SBC1 and at least one sub-optimal SBC sites 
remains, but it is defective when only one SBC site is present. This result is consistent with the second hypothesis and also suggests that the two binding sites are not equal. The optimal binding SBC sequence may dock the substrate on the adaptor and brings the suboptimal sites into close proximity; then the second SBC site may bind and orient the substrate into a proper conformation for ubiquitination. Our results also support the second hypothesis of CRL dimerization by providing an alternative solution to the first hypothesis. The flexible linker between the MATH and BTB domains provides numerous catalytic geometries for ubiquitination. Therefore, the flexibility of SPOP, along with dimerization, plays a major role in substrate orientation.

\subsection{FUTURE DIRECTIONS}

Our study raises many questions that require further examination. Biological approaches will be needed to characterize each SPOP-involved pathway and biochemical and structural biology analyses will be needed to explore the new hypotheses about CRLs that have emerged. The future directions can be summarized as follows.

Firstly, from our study of the SPOP ${ }^{\mathrm{MATH}}$-SBC interaction, we learned that a single substrate-binding domain can be used to map adaptor-recognition regions on substrates. This can be applied to other potential substrate adaptors to identify substrates or map binding sequences. In addition, because SPOP is related to cancer and is over expressed in kidney cancer, the structure of $\mathrm{SPOP}^{\mathrm{MATH}}-\mathrm{SBC}$ provides a molecular basis for SPOP-substrate interaction and will facilitate the design of new inhibitors and activators and potentially advance the development of novel anticancer drugs.

Secondly, as more and more BTB proteins are studied, many have been characterized as Cul3-binding proteins, that mediate the ubiquitination of specific substrate proteins. However, whether all BTB proteins function as bona fide $\mathrm{Cul} 3$ adaptors is unknown and why some BTBs bind Cul3 while others do not, despite conserved BTB folding, is still not clear. Our studies predicted the existence of a 3-box among the ten identified Cul3-binding BTBs, but not in most BTB-ZF transcription factors or T1-like BTB proteins. Thus, we speculate that BTB proteins require the 3-box to function as $\mathrm{Cul} 3$ adaptors. This theory needs to be further validated by studies of more BTB proteins. In addition, we cannot exclude the possibility that deletion of the 3-box interferes with Cul3 binding by affecting the local structure of the Cul3-binding surface of SPOP. Future studies of the SPOP-Cul3 complex might answer this question and provide more insight into the similarities and differences between the 3-box and F-box in regard to their interactions with cullin.

Finally, although our findings from this study suggest potential roles for flexibility and dimerization of CRLs, as described above, they do not provide a conclusive model. Further studies examining the E3 activity of $\mathrm{Cul} 3{ }^{\mathrm{SPOP}}$ with altered flexibility of SPOP will need to be pursued. The linker between the MATH and BTB domains regulates the flexibility of SPOP; thus, proline mutations or deletions can be introduced into the linker to restrain flexibility, and insertions can be introduced to relax 
the restraint. As for the two-site binding model, additional measurements, such as ITC (isothermal titration calorimetry) or mutational analysis, will be required to confirm the two-site binding and exclude the possibility that the binding of one substrate blocks that of the other. In addition, in the case of $\mathrm{SCF}^{\mathrm{Cdc} 4}$, dimerization is essential for ubiquitination in vivo but not in vitro [65]. This finding may reflect the high concentrations of enzymes used in the in vitro ubiquitination assay. It is also possible that dimerization affects something that is not included in the in vitro assay, such as enzymes in the NEDD8 pathway, COP9 signalosome, and post-translational modification systems that regulate CRL activity. Future studies may reveal additional functions of dimerization by addressing these possibilities. 


\section{LIST OF REFERENCES}

1. Johnson, E.S., et al., Ubiquitin as a degradation signal. Embo J, 1992. 11(2): p. 497-505.

2. Pickart, C.M. and M.J. Eddins, Ubiquitin: structures, functions, mechanisms. Biochim Biophys Acta, 2004. 1695(1-3): p. 55-72.

3. Ciechanover, A., The ubiquitin-proteasome pathway: on protein death and cell life. Embo J, 1998. 17(24): p. 7151-60.

4. Zhang, Q., et al., A hedgehog-induced BTB protein modulates hedgehog signaling by degrading Ci/Gli transcription factor. Dev Cell, 2006. 10(6): p. 719-29.

5. Jaakkola, P., et al., Targeting of HIF-alpha to the von Hippel-Lindau ubiquitylation complex by O2-regulated prolyl hydroxylation. Science, 2001. 292(5516): p. 468-72.

6. Kobayashi, M. and M. Yamamoto, Molecular mechanisms activating the Nrf2-Keapl pathway of antioxidant gene regulation. Antioxid Redox Signal, 2005. 7(3-4): p. 385-94.

7. Wu, C.J., et al., NEMO is a sensor of Lys 63-linked polyubiquitination and functions in NF-kappaB activation. Nat Cell Biol, 2006. 8(4): p. 398-406.

8. Chen, Z.J., Ubiquitin signalling in the NF-kappaB pathway. Nat Cell Biol, 2005. 7(8): p. 758-65.

9. Wertz, I.E., et al., De-ubiquitination and ubiquitin ligase domains of A20 downregulate NF-kappaB signalling. Nature, 2004. 430(7000): p. 694-9.

10. Angers, S., et al., The KLHL12-Cullin-3 ubiquitin ligase negatively regulates the Wnt-beta-catenin pathway by targeting Dishevelled for degradation. Nat Cell Biol, 2006. 8(4): p. 348-57.

11. Marikawa, Y. and R.P. Elinson, Beta-TrCP is a negative regulator of Wnt/beta-catenin signaling pathway and dorsal axis formation in Xenopus embryos. Mech Dev, 1998. 77(1): p. 75-80.

12. Scrima, A., et al., Structural basis of UV DNA-damage recognition by the DDB1-DDB2 complex. Cell, 2008. 135(7): p. 1213-23.

13. Angers, S., et al., Molecular architecture and assembly of the DDB1-CUL4A ubiquitin ligase machinery. Nature, 2006. 443(7111): p. 590-3.

14. Kuo, M.L., et al., N-terminal polyubiquitination and degradation of the Arf tumor suppressor. Genes Dev, 2004. 18(15): p. 1862-74. 
15. Clegg, H.V., K. Itahana, and Y. Zhang, Unlocking the Mdm2-p53 loop: ubiquitin is the key. Cell Cycle, 2008. 7(3): p. 287-92.

16. Brooks, C.L. and W. Gu, p53 ubiquitination: Mdm2 and beyond. Mol Cell, 2006. 21(3): p. 307-15.

17. Hoeller, D., et al., Regulation of ubiquitin-binding proteins by monoubiquitination. Nat Cell Biol, 2006. 8(2): p. 163-9.

18. Haglund, K., P.P. Di Fiore, and I. Dikic, Distinct monoubiquitin signals in receptor endocytosis. Trends Biochem Sci, 2003. 28(11): p. 598-603.

19. Tenno, T., et al., Structural basis for distinct roles of Lys63-and Lys48-linked polyubiquitin chains. Genes Cells, 2004. 9(10): p. 865-75.

20. Thrower, J.S., et al., Recognition of the polyubiquitin proteolytic signal. Embo J, 2000. 19(1): p. 94-102.

21. Johnson, E.S., et al., A proteolytic pathway that recognizes ubiquitin as a degradation signal. J Biol Chem, 1995. 270(29): p. 17442-56.

22. Duncan, L.M., et al., Lysine-63-linked ubiquitination is required for endolysosomal degradation of class I molecules. Embo J, 2006. 25(8): p. 1635-45.

23. Galan, J.M. and R. Haguenauer-Tsapis, Ubiquitin lys63 is involved in ubiquitination of a yeast plasma membrane protein. Embo J, 1997. 16(19): p. 5847-54.

24. Huang, D.T., et al., Basis for a ubiquitin-like protein thioester switch toggling E1-E2 affinity. Nature, 2007. 445(7126): p. 394-8.

25. Huang, D.T., et al., Structural basis for recruitment of Ubc12 by an E2 binding domain in NEDD8's E1. Mol Cell, 2005. 17(3): p. 341-50.

26. Lois, L.M. and C.D. Lima, Structures of the SUMO E1 provide mechanistic insights into SUMO activation and E2 recruitment to E1. Embo J, 2005. 24(3): p. 439-51.

27. Pickart, C.M., Mechanisms underlying ubiquitination. Annu Rev Biochem, 2001. 70: p. 503-33.

28. Hershko, A. and A. Ciechanover, The ubiquitin system. Annu Rev Biochem, 1998. 67: p. 425-79.

29. Huang, L., et al., Structure of an E6AP-UbcH7 complex: insights into ubiquitination by the E2-E3 enzyme cascade. Science, 1999. 286(5443): p. 13216. 
30. Huibregtse, J.M., et al., A family of proteins structurally and functionally related to the E6-AP ubiquitin-protein ligase. Proc Natl Acad Sci U S A, 1995. 92(7): p. 2563-7.

31. Dupont, S., et al., Germ-layer specification and control of cell growth by Ectodermin, a Smad4 ubiquitin ligase. Cell, 2005. 121(1): p. 87-99.

32. Yamashita, M., et al., Ubiquitin ligase Smurf1 controls osteoblast activity and bone homeostasis by targeting MEKK2 for degradation. Cell, 2005. 121(1): p. 101-13.

33. Ingham, R.J., G. Gish, and T. Pawson, The Nedd4 family of E3 ubiquitin ligases: functional diversity within a common modular architecture. Oncogene, 2004. 23(11): p. 1972-84.

34. Chen, C. and L.E. Matesic, The Nedd4-like family of E3 ubiquitin ligases and cancer. Cancer Metastasis Rev, 2007. 26(3-4): p. 587-604.

35. Joazeiro, C.A., et al., The tyrosine kinase negative regulator $\mathrm{c}$-Cbl as a RING-type, E2-dependent ubiquitin-protein ligase. Science, 1999. 286(5438): p. 309-12.

36. Lorick, K.L., et al., RING fingers mediate ubiquitin-conjugating enzyme E2-dependent ubiquitination. Proc Natl Acad Sci U S A, 1999. 96(20): p. 11364-9.

37. Zheng, N., et al., Structure of a c-Cbl-UbcH7 complex: RING domain function in ubiquitin-protein ligases. Cell, 2000. 102(4): p. 533-9.

38. Willems, A.R., M. Schwab, and M. Tyers, A hitchhiker's guide to the cullin ubiquitin ligases: SCF and its kin. Biochim Biophys Acta, 2004. 1695(1-3): p. 133-70.

39. Cardozo, T. and M. Pagano, The SCF ubiquitin ligase: insights into a molecular machine. Nat Rev Mol Cell Biol, 2004. 5(9): p. 739-51.

40. Petroski, M.D. and R.J. Deshaies, Function and regulation of cullin-RING ubiquitin ligases. Nat Rev Mol Cell Biol, 2005. 6(1): p. 9-20.

41. Merlet, J., et al., Regulation of cullin-RING E3 ubiquitin-ligases by neddylation and dimerization. Cell Mol Life Sci, 2009 (in press).

42. Page, A.M. and P. Hieter, The anaphase-promoting complex: new subunits and regulators. Annu Rev Biochem, 1999. 68: p. 583-609.

43. Peters, J.M., The anaphase promoting complex/cyclosome: a machine designed to destroy. Nat Rev Mol Cell Biol, 2006. 7(9): p. 644-56. 
44. Wu, G., et al., Structure of a beta-TrCP1-Skp1-beta-catenin complex: destruction motif binding and lysine specificity of the SCF(beta-TrCP1) ubiquitin ligase. Mol Cell, 2003. 11(6): p. 1445-56.

45. Zheng, N., et al., Structure of the Cul1-Rbx1-Skp1-F boxSkp2 SCF ubiquitin ligase complex. Nature, 2002. 416(6882): p. 703-9.

46. Schulman, B.A., et al., Insights into SCF ubiquitin ligases from the structure of the Skp1-Skp2 complex. Nature, 2000. 408(6810): p. 381-6.

47. Min, J.H., et al., Structure of an HIF-1alpha -pVHL complex: hydroxyproline recognition in signaling. Science, 2002. 296(5574): p. 1886-9.

48. Orlicky, S., et al., Structural basis for phosphodependent substrate selection and orientation by the SCFCdc4 ubiquitin ligase. Cell, 2003. 112(2): p. 243-56.

49. Mahrour, N., et al., Characterization of cullin-box sequences that direct recruitment of Cul2-Rbx1 and Cul5-Rbx2 modules to elongin BC-based ubiquitin ligases. J Biol Chem, 2008. 283(12): p. 8005-13.

50. Kamura, T., et al., VHL-box and SOCS-box domains determine binding specificity for Cul2-Rbx1 and Cul5-Rbx2 modules of ubiquitin ligases. Genes Dev, 2004. 18(24): p. 3055-65.

51. Zollman, S., et al., The BTB domain, found primarily in zinc finger proteins, defines an evolutionarily conserved family that includes several developmentally regulated genes in Drosophila. Proc Natl Acad Sci U S A, 1994. 91(22): p. 10717-21.

52. Pintard, L., A. Willems, and M. Peter, Cullin-based ubiquitin ligases: Cul3-BTB complexes join the family. Embo J, 2004. 23(8): p. 1681-7.

53. $\mathrm{Xu}, \mathrm{L}$., et al., BTB proteins are substrate-specific adaptors in an SCF-like modular ubiquitin ligase containing CUL-3. Nature, 2003. 425(6955): p. 316-21.

54. Krek, W., BTB proteins as henchmen of Cul3-based ubiquitin ligases. Nat Cell Biol, 2003. 5(11): p. 950-1.

55. Pintard, L., et al., The BTB protein MEL-26 is a substrate-specific adaptor of the CUL-3 ubiquitin-ligase. Nature, 2003. 425(6955): p. 311-6.

56. Geyer, R., et al., BTB/POZ domain proteins are putative substrate adaptors for cullin 3 ubiquitin ligases. Mol Cell, 2003. 12(3): p. 783-90.

57. Furukawa, M., et al., Targeting of protein ubiquitination by BTB-Cullin 3-Roc1 ubiquitin ligases. Nat Cell Biol, 2003. 5(11): p. 1001-7. 
58. Liu, J., et al., Analysis of drosophila segmentation network identifies a JNK pathway factor overexpressed in kidney cancer. Science, 2009 (in press).

59. Sumara, I., et al., A Cul3-based E3 ligase removes Aurora B from mitotic chromosomes, regulating mitotic progression and completion of cytokinesis in human cells. Dev Cell, 2007. 12(6): p. 887-900.

60. Allen, E., et al., Gigaxonin-controlled degradation of MAP1B light chain is critical to neuronal survival. Nature, 2005. 438(7065): p. 224-8.

61. Cullinan, S.B., et al., The Keap1-BTB protein is an adaptor that bridges Nrf2 to a Cul3-based E3 ligase: oxidative stress sensing by a Cul3-Keapl ligase. Mol Cell Biol, 2004. 24(19): p. 8477-86.

62. Hao, B., et al., Structural basis of the Cks1-dependent recognition of p27(Kip1) by the SCF(Skp2) ubiquitin ligase. Mol Cell, 2005. 20(1): p. 9-19.

63. Vlach, J., S. Hennecke, and B. Amati, Phosphorylation-dependent degradation of the cyclin-dependent kinase inhibitor p27. Embo J, 1997. 16(17): p. 5334-44.

64. Yu, Y., et al., Selective assembly of HIV-1 Vif-Cul5-ElonginB-ElonginC E3 ubiquitin ligase complex through a novel SOCS box and upstream cysteines. Genes Dev, 2004. 18(23): p. 2867-72.

65. Hao, B., et al., Structure of a Fbw7-Skp1-cyclin E complex: multisite-phosphorylated substrate recognition by SCF ubiquitin ligases. Mol Cell, 2007. 26(1): p. 131-43.

66. Hon, W.C., et al., Structural basis for the recognition of hydroxyproline in HIF-1 alpha by $p V H L$. Nature, 2002. 417(6892): p. 975-8.

67. Bergamin, E., J. Wu, and S.R. Hubbard, Structural basis for phosphotyrosine recognition by suppressor of cytokine signaling-3. Structure, 2006. 14(8): $\mathrm{p}$. 1285-92.

68. Li, T., et al., Structure of DDB1 in complex with a paramyxovirus V protein: viral hijack of a propeller cluster in ubiquitin ligase. Cell, 2006. 124(1): p. 105-17.

69. Duda, D.M., et al., Structural insights into NEDD8 activation of cullin-RING ligases: conformational control of conjugation. Cell, 2008. 134(6): p. 995-1006.

70. Yamoah, K., et al., Autoinhibitory regulation of SCF-mediated ubiquitination by human cullin 1's C-terminal tail. Proc Natl Acad Sci U S A, 2008. 105(34): p. 12230-5.

71. Saha, A. and R.J. Deshaies, Multimodal activation of the ubiquitin ligase SCF by Nedd8 conjugation. Mol Cell, 2008. 32(1): p. 21-31. 
72. Pan, Z.Q., et al., Nedd8 on cullin: building an expressway to protein destruction. Oncogene, 2004. 23(11): p. 1985-97.

73. Kerscher, O., R. Felberbaum, and M. Hochstrasser, Modification of proteins by ubiquitin and ubiquitin-like proteins. Annu Rev Cell Dev Biol, 2006. 22: p. 159-80.

74. Tateishi, K., et al., The NEDD8 system is essential for cell cycle progression and morphogenetic pathway in mice. J Cell Biol, 2001. 155(4): p. 571-9.

75. Jones, D., et al., Functional and phylogenetic analysis of the ubiquitylation system in Caenorhabditis elegans: ubiquitin-conjugating enzymes, ubiquitin-activating enzymes, and ubiquitin-like proteins. Genome Biol, 2002. 3(1): p.

RESEARCH0002.

76. Kurz, T., et al., Cytoskeletal regulation by the Nedd8 ubiquitin-like protein modification pathway. Science, 2002. 295(5558): p. 1294-8.

77. Ou, C.Y., et al., Distinct protein degradation mechanisms mediated by Cull and Cul3 controlling Ci stability in Drosophila eye development. Genes Dev, 2002. 16(18): p. 2403-14.

78. del Pozo, J.C., et al., AXR1-ECR1-dependent conjugation of RUB1 to the Arabidopsis Cullin AtCUL1 is required for auxin response. Plant Cell, 2002. 14(2): p. 421-33.

79. Morimoto, M., et al., Modification of cullin-1 by ubiquitin-like protein Nedd8 enhances the activity of SCF(skp2) toward p27(kip1). Biochem Biophys Res Commun, 2000. 270(3): p. 1093-6.

80. Read, M.A., et al., Nedd8 modification of cul-1 activates $S C F($ beta(TrCP))dependent ubiquitination of IkappaBalpha. Mol Cell Biol, 2000. 20(7): p. 2326-33.

81. Wu, K., A. Chen, and Z.Q. Pan, Conjugation of Nedd8 to CUL1 enhances the ability of the ROC1-CUL1 complex to promote ubiquitin polymerization. J Biol Chem, 2000. 275(41): p. 32317-24.

82. Amir, R.E., K. Iwai, and A. Ciechanover, The NEDD8 pathway is essential for $S C F$ (beta-TrCP)-mediated ubiquitination and processing of the NF-kappa B precursor p105. J Biol Chem, 2002. 277(26): p. 23253-9.

83. Liu, J., et al., NEDD8 modification of CUL1 dissociates p120(CAND1), an inhibitor of CUL1-SKP1 binding and SCF ligases. Mol Cell, 2002. 10(6): p. 1511-8.

84. Zheng, J., et al., CAND1 binds to unneddylated CUL1 and regulates the formation of SCF ubiquitin E3 ligase complex. Mol Cell, 2002. 10(6): p. 1519-26. 
85. Goldenberg, S.J., et al., Structure of the Cand1-Cul1-Roc1 complex reveals regulatory mechanisms for the assembly of the multisubunit cullin-dependent ubiquitin ligases. Cell, 2004. 119(4): p. 517-28.

86. Tang, X., et al., Suprafacial orientation of the SCFCdc4 dimer accommodates multiple geometries for substrate ubiquitination. Cell, 2007. 129(6): p. 1165-76.

87. Zipper, L.M. and R.T. Mulcahy, The Keap1 BTB/POZ dimerization function is required to sequester Nrf2 in cytoplasm. J Biol Chem, 2002. 277(39): p. 36544-52.

88. Welcker, M. and B.E. Clurman, $F b w 7 / h C D C 4$ dimerization regulates its substrate interactions. Cell Div, 2007. 2: p. 7.

89. Chew, E.H., et al., Characterization of cullin-based E3 ubiquitin ligases in intact mammalian cells--evidence for cullin dimerization. Cell Signal, 2007. 19(5): p. 1071-80.

90. Berthold, J., et al., Characterization of RhoBTB-dependent Cul3 ubiquitin ligase complexes--evidence for an autoregulatory mechanism. Exp Cell Res, 2008. 314(19): p. 3453-65.

91. Wilkins, A. and C.L. Carpenter, Regulation of RhoBTB2 by the Cul3 ubiquitin ligase complex. Methods Enzymol, 2008. 439: p. 103-9.

92. Dow, M.R. and P.E. Mains, Genetic and molecular characterization of the caenorhabditis elegans gene, mel-26, a postmeiotic negative regulator of mei-1, a meiotic-specific spindle component. Genetics, 1998. 150(1): p. 119-28.

93. Ravid, T. and M. Hochstrasser, Diversity of degradation signals in the ubiquitinproteasome system. Nat Rev Mol Cell Biol, 2008. 9(9): p. 679-90.

94. Skowyra, D., et al., F-box proteins are receptors that recruit phosphorylated substrates to the SCF ubiquitin-ligase complex. Cell, 1997. 91(2): p. 209-19.

95. Hart, M., et al., The F-box protein beta-TrCP associates with phosphorylated beta-catenin and regulates its activity in the cell. Curr Biol, 1999. 9(4): p. 207-10.

96. Margottin-Goguet, F., et al., Prophase destruction of Emil by the $S C F$ (betaTrCP/Slimb) ubiquitin ligase activates the anaphase promoting complex to allow progression beyond prometaphase. Dev Cell, 2003. 4(6): p. 813-26.

97. Babon, J.J., et al., The structure of SOCS3 reveals the basis of the extended SH2 domain function and identifies an unstructured insertion that regulates stability. Mol Cell, 2006. 22(2): p. 205-16. 
98. Bullock, A.N., et al., Crystal structure of the SOCS2-elongin C-elongin B complex defines a prototypical SOCS box ubiquitin ligase. Proc Natl Acad Sci U S A, 2006. 103(20): p. 7637-42.

99. Bullock, A.N., et al., Structure of the SOCS4-ElonginB/C complex reveals a distinct SOCS box interface and the molecular basis for SOCS-dependent EGFR degradation. Structure, 2007. 15(11): p. 1493-504.

100. Ivan, M., et al., HIFalpha targeted for VHL-mediated destruction by proline hydroxylation: implications for O2 sensing. Science, 2001. 292(5516): p. 464-8.

101. Petroski, M.D. and R.J. Deshaies, Context of multiubiquitin chain attachment influences the rate of Sicl degradation. Mol Cell, 2003. 11(6): p. 1435-44.

102. Brown, N.R., et al., The structural basis for specificity of substrate and recruitment peptides for cyclin-dependent kinases. Nat Cell Biol, 1999. 1(7): p. 438-43.

103. Winston, J.T., et al., The SCFbeta-TRCP-ubiquitin ligase complex associates specifically with phosphorylated destruction motifs in IkappaBalpha and betacatenin and stimulates IkappaBalpha ubiquitination in vitro. Genes Dev, 1999. 13(3): p. 270-83.

104. Orian, A., et al., SCF(beta)(-TrCP) ubiquitin ligase-mediated processing of NFkappaB p105 requires phosphorylation of its $C$-terminus by IkappaB kinase. Embo J, 2000. 19(11): p. 2580-91.

105. Jiang, J. and G. Struhl, Regulation of the Hedgehog and Wingless signalling pathways by the F-box/WD40-repeat protein Slimb. Nature, 1998. 391(6666): p. 493-6.

106. Latres, E., D.S. Chiaur, and M. Pagano, The human F box protein beta-Trcp associates with the Cul1/Skpl complex and regulates the stability of beta-catenin. Oncogene, 1999. 18(4): p. 849-54.

107. Stogios, P.J., et al., Sequence and structural analysis of BTB domain proteins. Genome Biol, 2005. 6(10): p. R82.

108. Zhang, L., N.Y. Villa, and G. McFadden, Interplay between poxviruses and the cellular ubiquitin/ubiquitin-like pathways. FEBS Letters, 2009. 583(4): p. 607-14.

109. Ahmad, K.F., C.K. Engel, and G.G. Prive, Crystal structure of the BTB domain from PLZF. Proc Natl Acad Sci U S A, 1998. 95(21): p. 12123-8.

110. Ahmad, K.F., et al., Mechanism of SMRT corepressor recruitment by the BCL6 BTB domain. Mol Cell, 2003. 12(6): p. 1551-64. 
111. Bayon, Y., et al., KCTD5, a putative substrate adaptor for cullin3 ubiquitin ligases. Febs J, 2008. 275(15): p. 3900-10.

112. Ghetu, A.F., et al., Structure of a BCOR corepressor peptide in complex with the BCL6 BTB domain dimer. Mol Cell, 2008. 29(3): p. 384-91.

113. Stogios, P.J., L. Chen, and G.G. Prive, Crystal structure of the BTB domain from the LRF/ZBTB7 transcriptional regulator. Protein Sci, 2007. 16(2): p. 336-42.

114. Melnick, A., et al., Critical residues within the BTB domain of PLZF and Bcl-6 modulate interaction with corepressors. Mol Cell Biol, 2002. 22(6): p. 1804-18.

115. Melnick, A., et al., In-depth mutational analysis of the promyelocytic leukemia zinc finger BTB/POZ domain reveals motifs and residues required for biological and transcriptional functions. Mol Cell Biol, 2000. 20(17): p. 6550-67.

116. Kwon, J.E., et al., BTB domain-containing speckle-type POZ protein (SPOP) serves as an adaptor of Daxx for ubiquitination by Cul3-based ubiquitin ligase. $\mathrm{J}$ Biol Chem, 2006. 281(18): p. 12664-72.

117. Lo, S.C., et al., Structure of the Keap1:Nrf2 interface provides mechanistic insight into Nrf2 signaling. Embo J, 2006. 25(15): p. 3605-17.

118. Vogel, C., et al., Structure, function and evolution of multidomain proteins. Curr Opin Struct Biol, 2004. 14(2): p. 208-16.

119. Gingerich, D.J., et al., Large-scale, lineage-specific expansion of a bric-abrac/tramtrack/broad complex ubiquitin-ligase gene family in rice. Plant Cell, 2007. 19(8): p. 2329-48.

120. Nagai, Y., et al., Identification of a novel nuclear speckle-type protein, SPOP. FEBS Lett, 1997. 418(1-2): p. 23-6.

121. Thompson, M.C., et al., Genomics identifies medulloblastoma subgroups that are enriched for specific genetic alterations. J Clin Oncol, 2006. 24(12): p. 1924-31.

122. Hernandez-Munoz, I., et al., Stable X chromosome inactivation involves the PRC1 Polycomb complex and requires histone MACROH2A1 and the CULLIN3/SPOP ubiquitin E3 ligase. Proc Natl Acad Sci U S A, 2005. 102(21): p. 7635-40.

123. Takahashi, I., Y. Kameoka, and K. Hashimoto, MacroH2A1.2 binds the nuclear protein Spop. Biochim Biophys Acta, 2002. 1591(1-3): p. 63-8.

124. La, M., et al., Daxx-mediated transcriptional repression of MMP1 gene is reversed by SPOP. Biochem Biophys Res Commun, 2004. 320(3): p. 760-5. 
125. Kent, D., E.W. Bush, and J.E. Hooper, Roadkill attenuates Hedgehog responses through degradation of Cubitus interruptus. Development, 2006. 133(10): p. 2001-10.

126. Di Marcotullio, L., et al., Multiple ubiquitin-dependent processing pathways regulate hedgehog/gli signaling: implications for cell development and tumorigenesis. Cell Cycle, 2007. 6(4): p. 390-3.

127. Otwinowski, Z. and W. Minor, Processing of X-ray Diffraction Data Collected in Oscillation Mode, in Methods in Enzymology, Macromolecular Crystallography, part A, C.W. Carter and R.M. Sweet, Editors. 1997. p. 307-26.

128. Pahler, A., J.L. Smith, and W.A. Hendrickson, A probability representation for phase information from multiwavelength anomalous dispersion. Acta Crystallogr A, 1990. 46 ( Pt 7): p. 537-40.

129. Terwilliger, T.C. and J. Berendzen, Automated MAD and MIR structure solution. Acta Crystallogr D Biol Crystallogr, 1999. 55(Pt 4): p. 849-61.

130. Jones, T.A., et al., Improved methods for building protein models in electron density maps and the location of errors in these models. Acta Crystallogr A, 1991. 47(Pt 2): p. 110-19.

131. Brünger, A.T., et al., Crystallography \& NMR system: a new software suite for macromolecular structure determination. Acta Crystallogr D Biol Crystallogr, 1998. 54(Pt 5): p. 905-21.

132. Morris, A.L., et al., Stereochemical quality of protein structure coordinates. Proteins, 1992. 12(4): p. 345-64.

133. Storoni, L.C., A.J. McCoy, and R.J. Read, Likelihood-enhanced fast rotation functions. Acta Crystallogr D Biol Crystallogr, 2004. 60(Pt 3): p. 432-8.

134. Perrakis, A., et al., ARP/wARP and molecular replacement. Acta Crystallogr $\mathrm{D}$ Biol Crystallogr, 2001. 57(Pt 10): p. 1445-50.

135. Murshudov, G.N., A.A. Vagin, and E.J. Dodson, Refinement of macromolecular structures by the maximum-likelihood method. Acta Crystallogr D Biol Crystallogr, 1997. 53(Pt 3): p. 240-55.

136. Emsley, P. and K. Cowtan, Coot: model-building tools for molecular graphics. Acta Crystallogr D Biol Crystallogr, 2004. 60(Pt 12 Pt 1): p. 2126-32.

137. Putnam, C.D., et al., X-ray solution scattering (SAXS) combined with crystallography and computation: defining accurate macromolecular structures, conformations and assemblies in solution. Q Rev Biophys, 2007. 40(3): $\mathrm{p}$. 191-285. 
138. Guinier, A., et al., Small-Angle Scattering of X-rays. 1955, New York: Wiley.

139. Svergun, D., Determination of the regularization parameter in indirect-transform methods using perceptual criteria. J Appl Crystal, 1992. 28: p. 768-73.

140. Pelikan M., M. Hammel, Structure and flexibility within proteins as identified through small angle X-ray scattering. Gen Physiol and Biophys, 2009 (in press).

141. Jiang, J., Regulation of Hh/Gli signaling by dual ubiquitin pathways. Cell Cycle, 2006. 5(21): p. 2457-63.

142. Zapata, J.M., et al., A diverse family of proteins containing tumor necrosis factor receptor-associated factor domains. J Biol Chem, 2001. 276(26): p. 24242-52.

143. Avela, K., et al., Gene encoding a new RING-B-box-Coiled-coil protein is mutated in mulibrey nanism. Nat Genet, 2000. 25(3): p. 298-301.

144. Saridakis, V., et al., Structure of the p53 binding domain of HAUSP/USP7 bound to Epstein-Barr nuclear antigen 1 implications for EBV-mediated immortalization. Mol Cell, 2005. 18(1): p. 25-36.

145. Sheng, Y., et al., Molecular recognition of $p 53$ and MDM2 by USP7/HAUSP. Nat Struct Mol Biol, 2006. 13(3): p. 285-91.

146. Hu, M., et al., Structural basis of competitive recognition of p53 and MDM2 by HAUSP/USP7: implications for the regulation of the p53-MDM2 pathway. PLoS Biol, 2006. 4(2): p. e27.

147. Park, Y.C., et al., Structural basis for self-association and receptor recognition of human TRAF2. Nature, 1999. 398(6727): p. 533-8.

148. McWhirter, S.M., et al., Crystallographic analysis of CD40 recognition and signaling by human TRAF2. Proc Natl Acad Sci U S A, 1999. 96(15): p. 8408-13.

149. Chung, J.Y., et al., Molecular basis for the unique specificity of TRAF6. Adv Exp Med Biol, 2007. 597: p. 122-30.

150. Ye, H., et al., Distinct molecular mechanism for initiating TRAF6 signalling. Nature, 2002. 418(6896): p. 443-7.

151. Ni, C.Z., et al., Molecular basis for CD40 signaling mediated by TRAF3. Proc Natl Acad Sci U S A, 2000. 97(19): p. 10395-9.

152. Thomas, J.H., Adaptive evolution in two large families of ubiquitin-ligase adapters in nematodes and plants. Genome Res, 2006. 16(8): p. 1017-30.

153. Perez-Torrado, R., D. Yamada, and P.A. Defossez, Born to bind: the BTB proteinprotein interaction domain. Bioessays, 2006. 28(12): p. 1194-202. 
154. Bomont, P., et al., The gene encoding gigaxonin, a new member of the cytoskeletal BTB/kelch repeat family, is mutated in giant axonal neuropathy. Nat Genet, 2000. 26(3): p. 370-4.

155. Wang, W., et al., Gigaxonin interacts with tubulin folding cofactor B and controls its degradation through the ubiquitin-proteasome pathway. Curr Biol, 2005. 15(22): p. 2050-5.

156. Zhang, D.D., Mechanistic studies of the Nrf2-Keapl signaling pathway. Drug Metab Rev, 2006. 38(4): p. 769-89.

157. Koiwai, K., et al., BPOZ-2 directly binds to eEF1A1 to promote eEF1A1 ubiquitylation and degradation and prevent translation. Genes Cells, 2008. 13(6): p. 593-607.

158. Salinas, G.D., et al., Actinfilin is a Cul3 substrate adaptor, linking GluR6 kainate receptor subunits to the ubiquitin-proteasome pathway. J Biol Chem, 2006. 281(52): p. 40164-73.

159. Huangfu, D. and K.V. Anderson, Signaling from Smo to Ci/Gli: conservation and divergence of Hedgehog pathways from Drosophila to vertebrates. Development, 2006. 133(1): p. 3-14.

160. Osterlund, T. and P. Kogerman, Hedgehog signalling: how to get from Smo to Ci and Gli. Trends Cell Biol, 2006. 16(4): p. 176-80.

161. Chen, C.H., et al., Nuclear trafficking of Cubitus interruptus in the transcriptional regulation of Hedgehog target gene expression. Cell, 1999. 98(3): p. 305-16.

162. Wang, Q.T. and R.A. Holmgren, Nuclear import of cubitus interruptus is regulated by hedgehog via a mechanism distinct from $\mathrm{Ci}$ stabilization and $\mathrm{Ci}$ activation. Development, 2000. 127(14): p. 3131-9.

163. Jia, J., et al., Phosphorylation by double-time/CKIepsilon and CKIalpha targets cubitus interruptus for Slimb/beta-TRCP-mediated proteolytic processing. Dev Cell, 2005. 9(6): p. 819-30.

164. Jin, J., et al., SCFbeta-TRCP links Chk1 signaling to degradation of the Cdc25A protein phosphatase. Genes Dev, 2003. 17(24): p. 3062-74.

165. Tempe, D., et al., Multisite protein kinase A and glycogen synthase kinase 3beta phosphorylation leads to Gli3 ubiquitination by SCFbetaTrCP. Mol Cell Biol, 2006. 26(11): p. 4316-26.

166. Wang, B. and Y. Li, Evidence for the direct involvement of \{beta $\}$ TrCP in Gli3 protein processing. Proc Natl Acad Sci U S A, 2006. 103(1): p. 33-8. 
167. Zhang, W., et al., Hedgehog-regulated Costal2-kinase complexes control phosphorylation and proteolytic processing of Cubitus interruptus. Dev Cell, 2005. 8(2): p. 267-78.

168. Smelkinson, M.G. and D. Kalderon, Processing of the Drosophila hedgehog signaling effector $\mathrm{Ci}-155$ to the repressor $\mathrm{Ci}-75$ is mediated by direct binding to the SCF component Slimb. Curr Biol, 2006. 16(1): p. 110-6.

169. McEwen, D.G. and M. Peifer, Puckered, a Drosophila MAPK phosphatase, ensures cell viability by antagonizing JNK-induced apoptosis. Development, 2005. 132(17): p. 3935-46.

170. Perlman, R., et al., TGF-beta-induced apoptosis is mediated by the adapter protein Daxx that facilitates JNK activation. Nat Cell Biol, 2001. 3(8): p. 708-14.

171. Torii, S., et al., Human Daxx regulates Fas-induced apoptosis from nuclear PML oncogenic domains (PODs). Embo J, 1999. 18(21): p. 6037-49.

172. Yang, X., et al., Daxx, a novel Fas-binding protein that activates JNK and apoptosis. Cell, 1997. 89(7): p. 1067-76.

173. Tang, J., et al., Critical role for Daxx in regulating Mdm2. Nat Cell Biol, 2006. 8(8): p. 855-62.

174. Wallach, D., et al., Tumor necrosis factor receptor and Fas signaling mechanisms. Annu Rev Immunol, 1999. 17: p. 331-67.

175. Hicke, L. and R. Dunn, Regulation of membrane protein transport by ubiquitin and ubiquitin-binding proteins. Annu Rev Cell Dev Biol, 2003. 19: p. 141-72.

176. Ronai, Z., Balancing Mdm2 - a Daxx-HAUSP matter. Nat Cell Biol, 2006. 8(8): p. $790-1$.

177. Song, M.S., et al., The deubiquitinylation and localization of PTEN are regulated by a HAUSP-PML network. Nature, 2008. 455(7214): p. 813-7.

178. Perry, J.A. and S. Kornbluth, Cdc25 and Wee1: analogous opposites? Cell Div, 2007. 2: p. 12.

179. Kee, Y., N. Lyon, and J.M. Huibregtse, The Rsp5 ubiquitin ligase is coupled to and antagonized by the Ubp2 deubiquitinating enzyme. Embo J, 2005. 24(13): p. 2414-24.

180. Kee, Y., et al., The deubiquitinating enzyme Ubp2 modulates Rsp5-dependent Lys63-linked polyubiquitin conjugates in Saccharomyces cerevisiae. J Biol Chem, 2006. 281(48): p. 36724-31. 
181. Crosas, B., et al., Ubiquitin chains are remodeled at the proteasome by opposing ubiquitin ligase and deubiquitinating activities. Cell, 2006. 127(7): p. 1401-13.

182. Lee, H.J., et al., HAUSP, a deubiquitinating enzyme for p53, is polyubiquitinated, polyneddylated, and dimerized. FEBS Lett, 2005. 579(21): p. 4867-72.

183. Stebbins, C.E., W.G. Kaelin, Jr., and N.P. Pavletich, Structure of the VHLElonginC-ElonginB complex: implications for VHL tumor suppressor function. Science, 1999. 284(5413): p. 455-61.

184. Shirane, M., et al., Down-regulation of p27(Kip1) by two mechanisms, ubiquitinmediated degradation and proteolytic processing. J Biol Chem, 1999. 274(20): p. 13886-93.

185. Spencer, E., J. Jiang, and Z.J. Chen, Signal-induced ubiquitination of IkappaBalpha by the F-box protein Slimb/beta-TrCP. Genes Dev, 1999. 13(3): p. 284-94.

186. Fuchs, S.Y., V.S. Spiegelman, and K.G. Kumar, The many faces of beta-TrCP E3 ubiquitin ligases: reflections in the magic mirror of cancer. Oncogene, 2004. 23(11): p. 2028-36.

187. Petroski, M.D. and R.J. Deshaies, Mechanism of lysine 48-linked ubiquitin-chain synthesis by the cullin-RING ubiquitin-ligase complex SCF-Cdc34. Cell, 2005. 123(6): p. 1107-20.

188. Minella, A.C., et al., Cyclin E phosphorylation regulates cell proliferation in hematopoietic and epithelial lineages in vivo. Genes Dev, 2008. 22(12): p. 1677-89.

189. Whitby, F.G., et al., Crystal structure of the human ubiquitin-like protein NEDD8 and interactions with ubiquitin pathway enzymes. J Biol Chem, 1998. 273(52): p. 34983-91.

190. Huang, D.T., et al., Ubiquitin-like protein activation. Oncogene, 2004. 23(11): p. 1958-71.

191. Huang, D.T., et al., E2-RING expansion of the NEDD8 cascade confers specificity to cullin modification. Mol Cell, 2009. 33(4): p. 483-95.

192. Walden, H., et al., The structure of the APPBP1-UBA3-NEDD8-ATP complex reveals the basis for selective ubiquitin-like protein activation by an E1. Mol Cell, 2003. 12(6): p. 1427-37.

193. Walden, H., M.S. Podgorski, and B.A. Schulman, Insights into the ubiquitin transfer cascade from the structure of the activating enzyme for NEDD8. Nature, 2003. 422(6929): p. 330-4. 
194. Huang, D.T., et al., Identification of conjugation specificity determinants unmasks vestigial preference for ubiquitin within the NEDD8 E2. Nat Struct Mol Biol, 2008. 15(3): p. 280-7. 


\section{APPENDIX A. SUPPLEMENTARY DATA FOR STRUCTURAL}

STUDIES OF SPOP-CUL3 UBIQUITIN LIGASE 


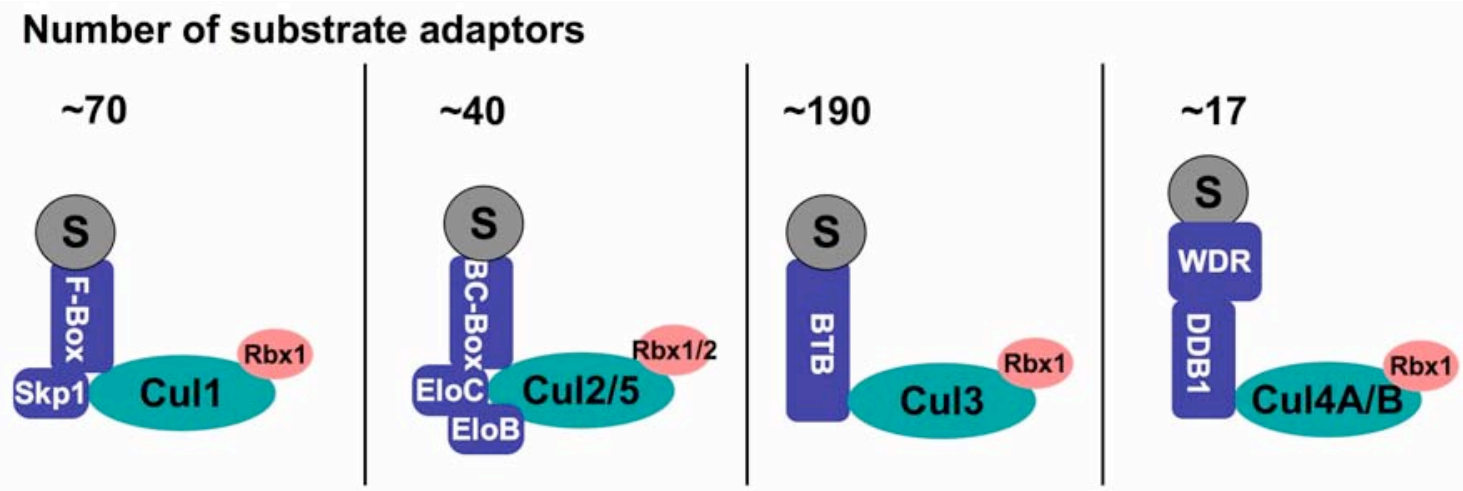

Skp1/Skp2/Cks1/p27
Skp1/Fbs1/sugar
Skp1//3-Trcp//_-Catenin
Skp1/TIR1/ask-1
Skp1/Cdc4/CPD
Skp1/Fbw7/cyclin E

\section{Crystal Structures}

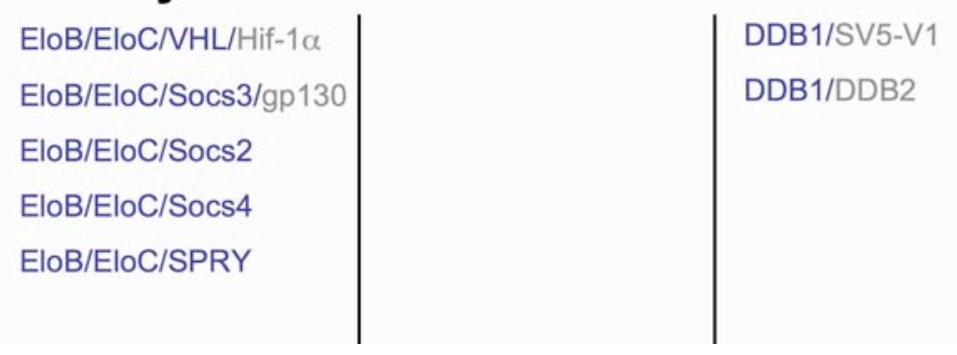

Figure A.1. List of crystal structures determined in each CRL subfamily. The numbers of potential substrate adaptors in Human are listed above. Protein names of substrate adaptors are colored in blue and the substrates are colored in grey. 


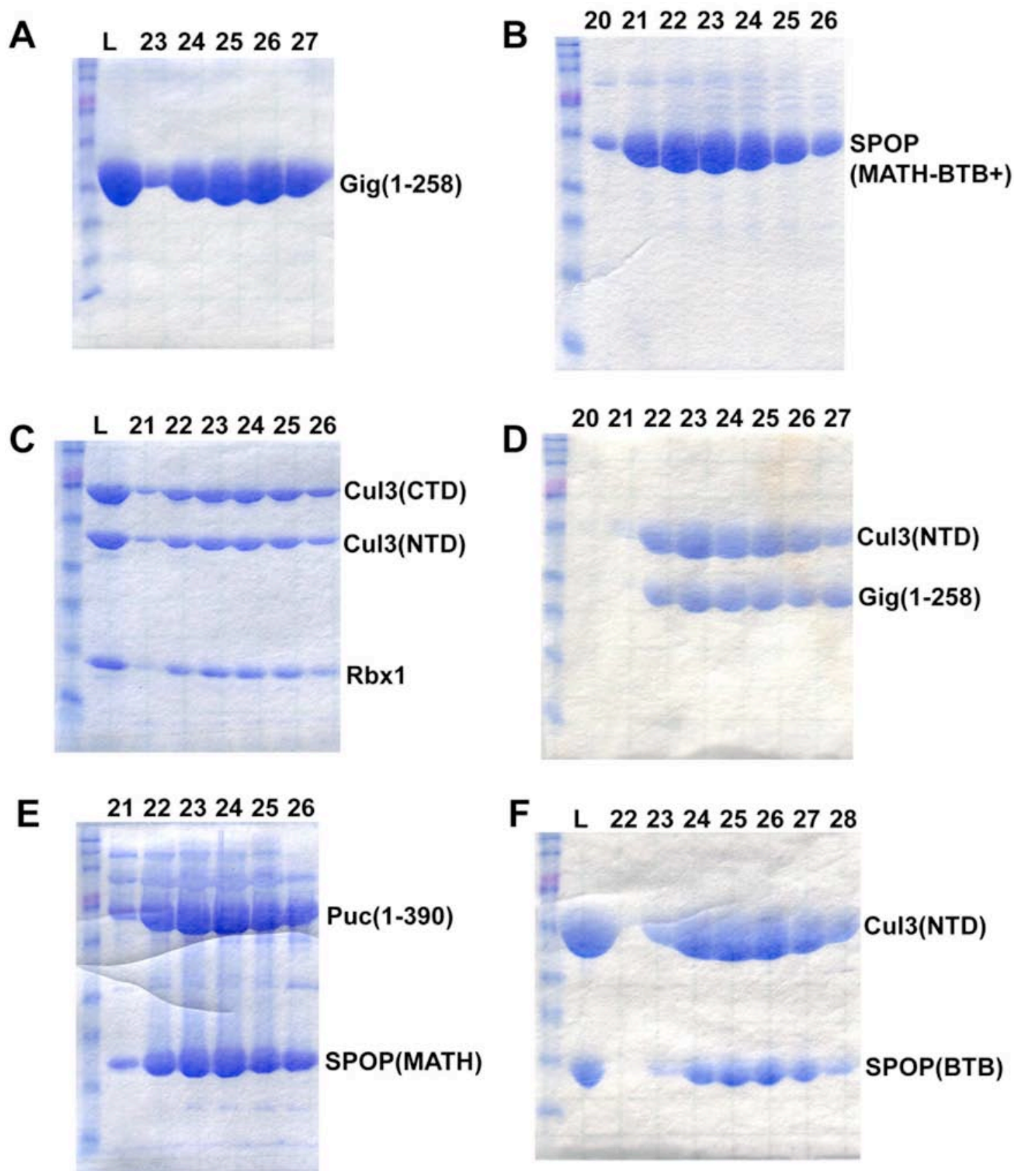

Figure A.2. Coomassie stain of representative purified proteins. On top of each gel is the fraction number of each sample eluted from SD200 $(25 \mathrm{ml})$ gel filtration column $(0.5 \mathrm{ml} /$ fraction). " $\mathrm{L}$ " is the sample loaded on FPLC. 


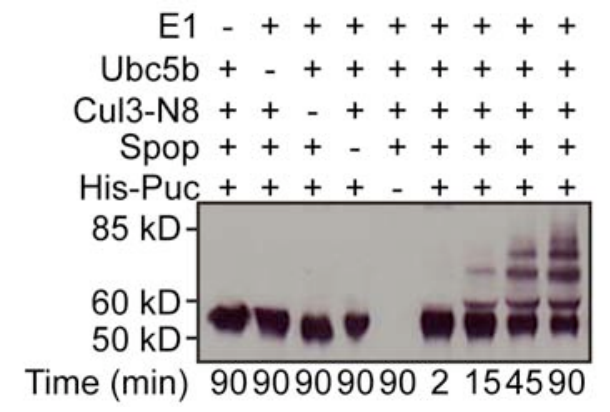

Figure A.3. Puc ubiquitination in vitro depends on SPOP, Cul3, E1 and E2 (Ubc5b). Anti-HisWestern blot detecting His-Puc after incubation with ubiquitin, MgATP, and E1 (UBA1), E2 (UbcH5b), SPOP, and/or Cul3 NEDD8 for the indicated times. 


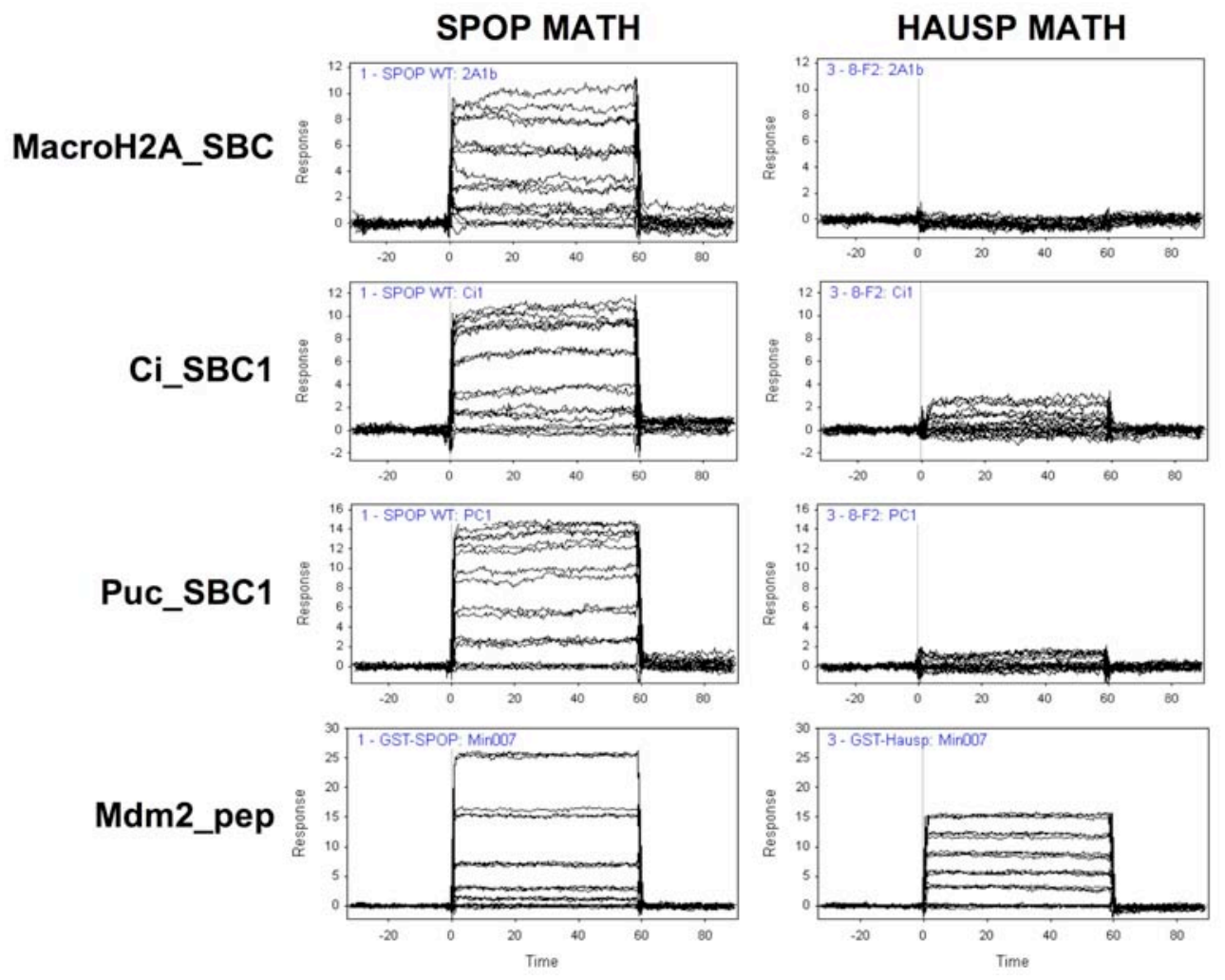

Figure A.4. SBC peptides bind SPOP MATH domain but do not bind HAUSP MATH domain. BIACORE sensograms for experiments analyzing SPOP ${ }^{\mathrm{MATH}}$ and HAUSP ${ }^{\mathrm{MATH}}$ binding to either MacroH2 $\mathrm{A}^{\mathrm{SBC}}, \mathrm{Ci}^{\mathrm{SBC} 2}, \mathrm{Puc}^{\mathrm{SBC} 1}$ or Mdm2 peptide. 
A

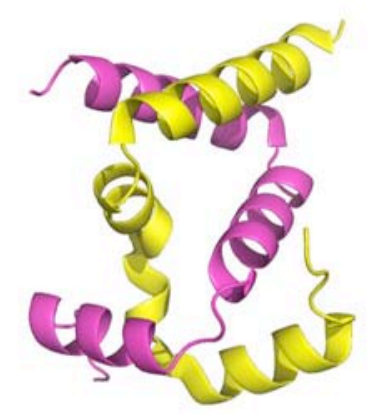

B

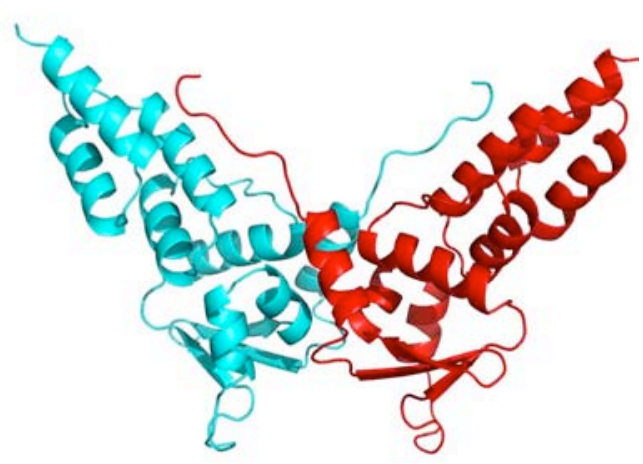

Figure A.5. Structure comparison of the D-domain and BTB domain. Dimeric Ddomain from $\mathrm{Cdc} 4$ is shown in yellow and magenta (2p63.pdb) (A). $\mathrm{SPOP}^{\mathrm{BTB}+}$ domain is show in cyan and red (B). Both structures have roughly two-fold symmetry but with distinct dimerization pattern. 
A

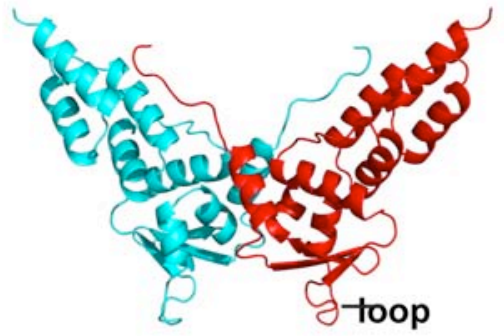

B

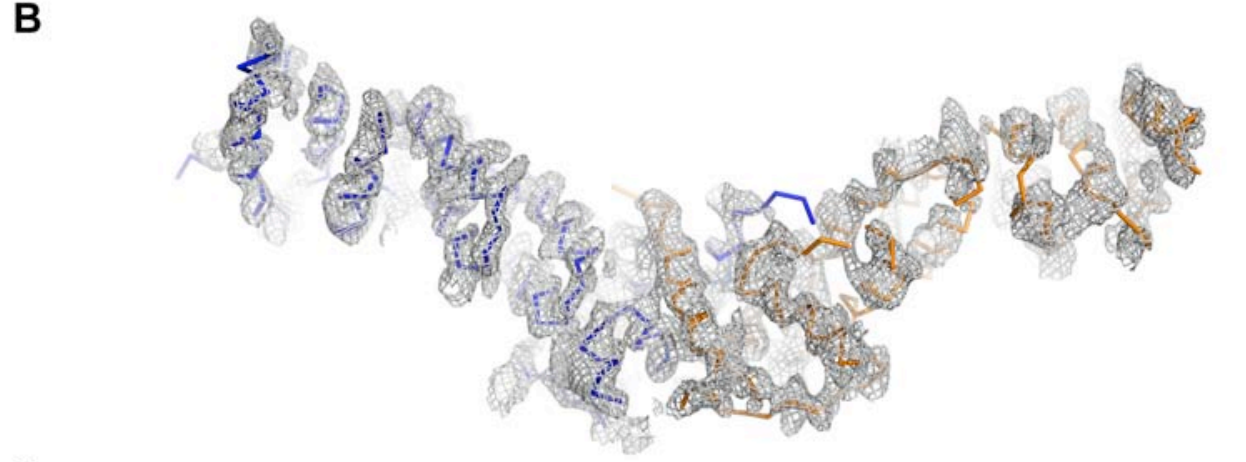

C

Anomalous Map (3.3A)

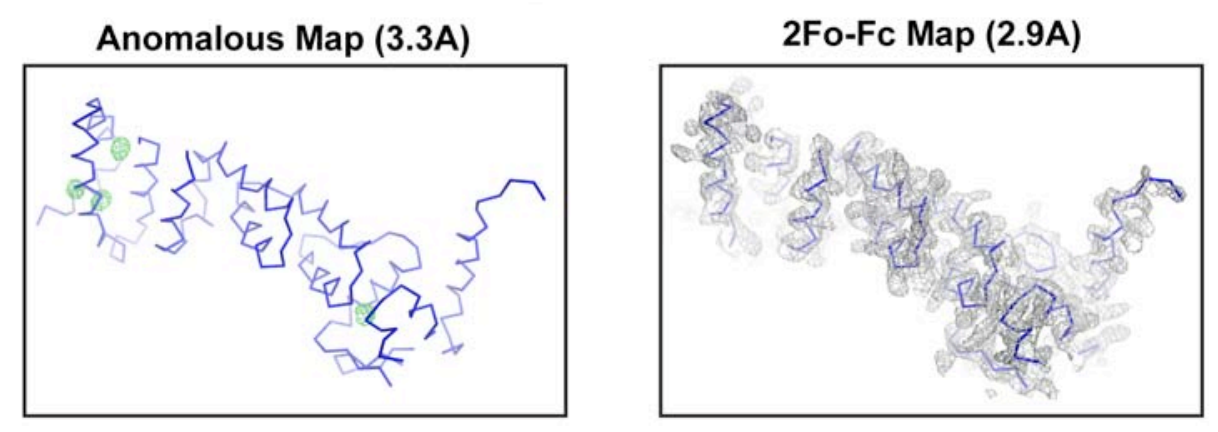

loop region

SPOP KAILAARSPVFS $\overline{\text { AMFENEMEESKKNRVEIN }}$

Gig KNILAAASPYIRTKLNYNPPKDDGSTYKIE

\begin{tabular}{ccc} 
Sample & Kd $(\mu \mathrm{M})$ & r.m.s.d. \\
\hline Gig & 0.29 & 0.0067 \\
Gig $\triangle$ DDGS & 0.23 & 0.0057
\end{tabular}

Figure A.6. Structure determination of Gig $^{1-258 \Delta \text { DDGS }}$. (A) To improve initial poorly diffracting crystals, a Gig construct was engineered based on the $\mathrm{SPOP}^{\mathrm{BTB}-3-\text { box }}$ structure (left). A 4-residue deletion was engineered, of a DDGS sequence corresponding to a loop in SPOP. Analytical Ultracentrifugation data show that Gig ${ }^{1-258 \triangle D D G S}$ maintains a Kd for dimerization similar to construct containing the loop. (B) Experimental SAD electron density over the $\mathrm{Gig}^{1-258 \Delta \mathrm{DDGS}}$ dimer (blue/orange) in the asymmetric unit, contoured at 1б. Residues C-terminal of the 3-box are modeled as polyalanine due to poor electron

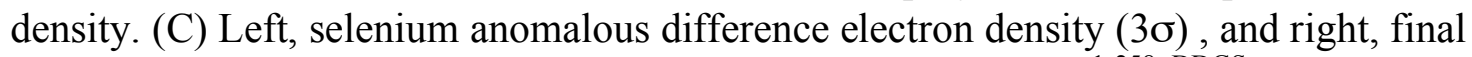
refined $2 \mathrm{Fo}-\mathrm{Fc}$ map $(1 \sigma)$ displayed over one protomer in the $\mathrm{Gig}^{1-258 \Delta \mathrm{DDGS}}$ asymmetric unit. 


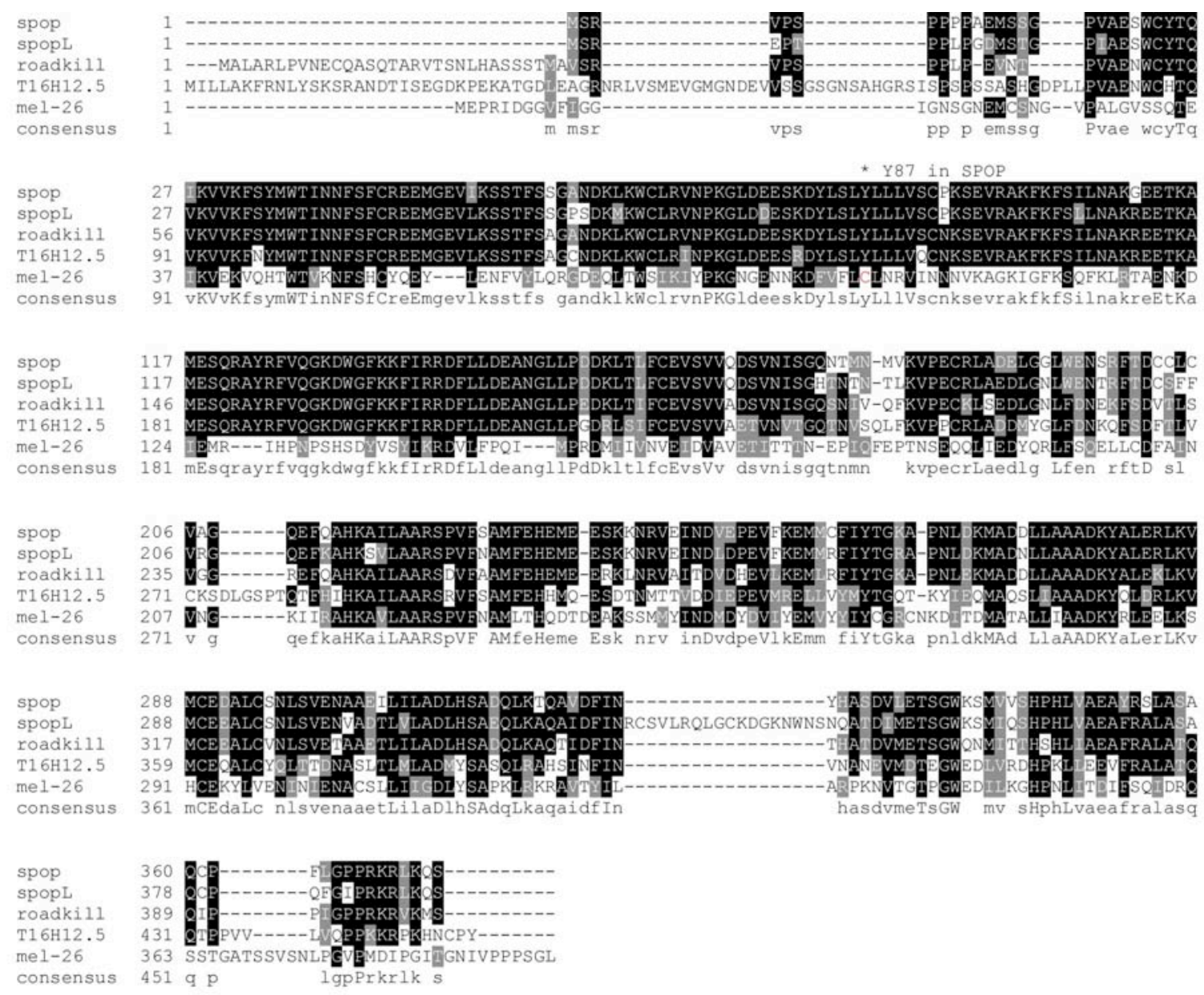

Figure A.7. Sequence alignment of SPOP from different species. 

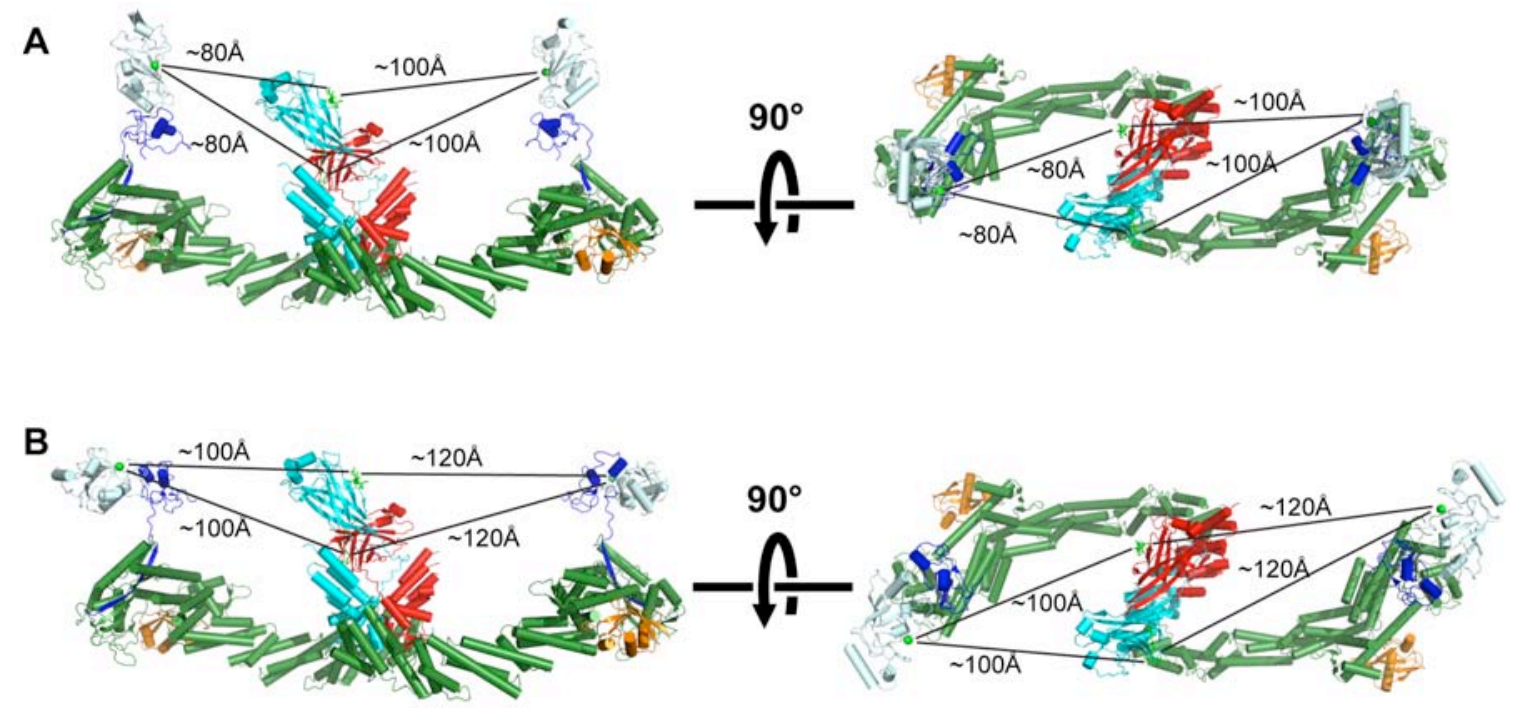

Figure A.8. Structural models of SPOP-Cul3 NEDD8-Rbx1-E2 complexes. Models were generated by superimposing the BTB domain of 1 SPOP protomer onto the corresponding region of Skp1 in the Skp1-Cul1-F-box ${ }^{\text {Skp2 }}$ structure (1LDK.pdb) [45]. The $4 \mathrm{HB}$ and $\alpha / \beta$ subdomains from the Cull $\mathrm{C}$-terminal domain were superimposed on the corresponding regions of the two conformations of NEDD $8 \sim \mathrm{Cul} 5^{\mathrm{ctd}}-\mathrm{Rbx} 1$

(1DQV.pdb) [69]. An E2 is modeled onto the Rbx1 RING domains based on the RINGE2 structure of c-Cbl-UbcH7 (1FBV.pdb) [37]. Dimerization, combined with multiple flexible hinges, allow many orientations between an E2 and the Puc ${ }^{\mathrm{SBC}}$ bound to a SPOPCul3 NEDD8-Rbx1 ligase. 

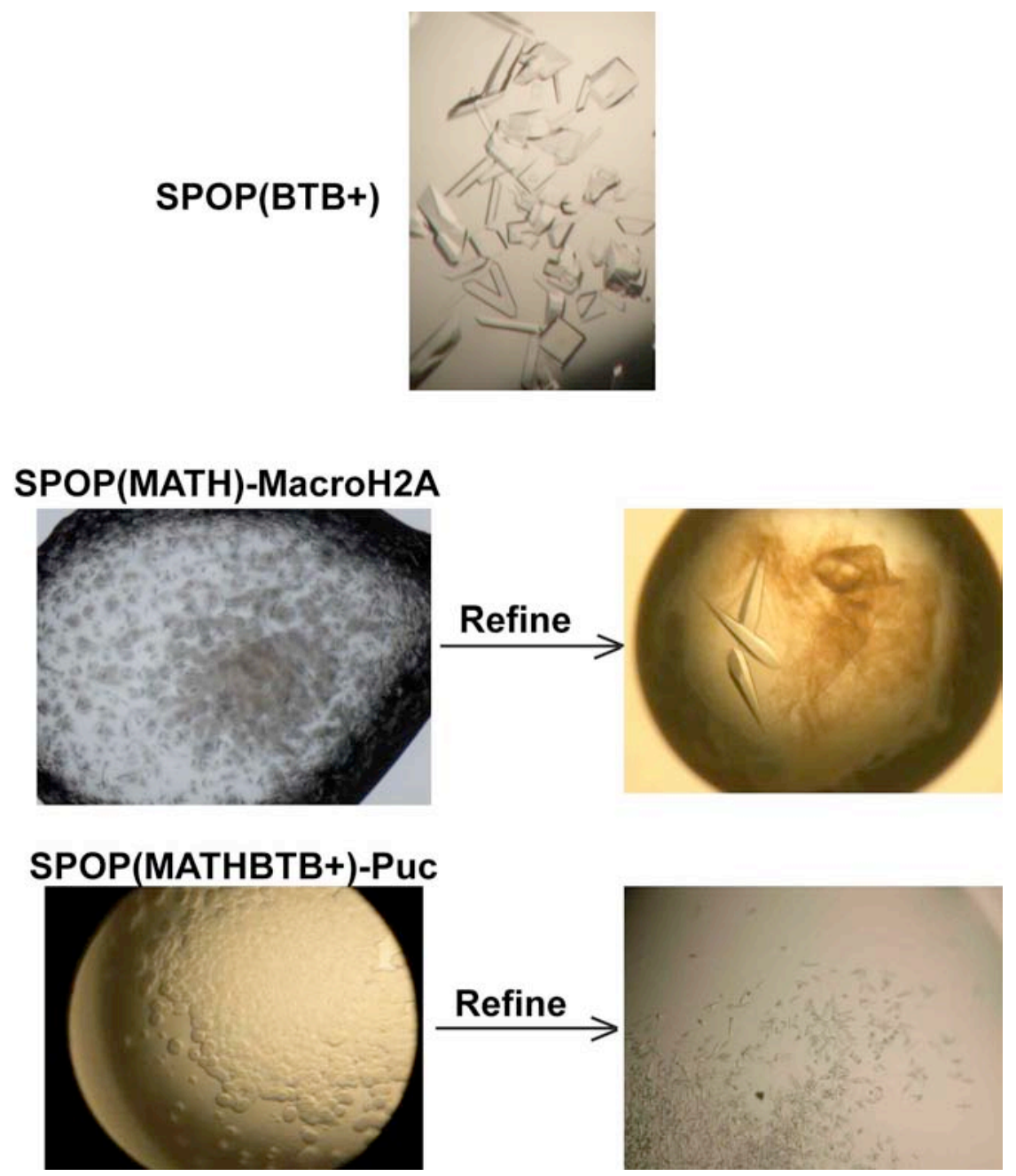

Figure A.9. Representative crystal pictures. 
A

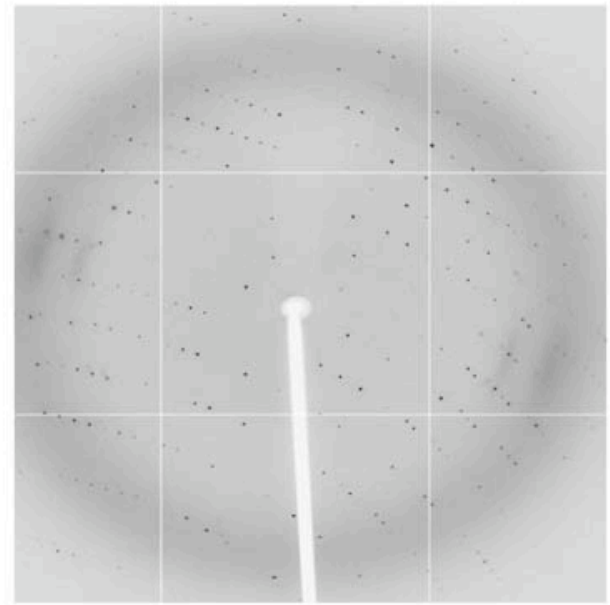

C

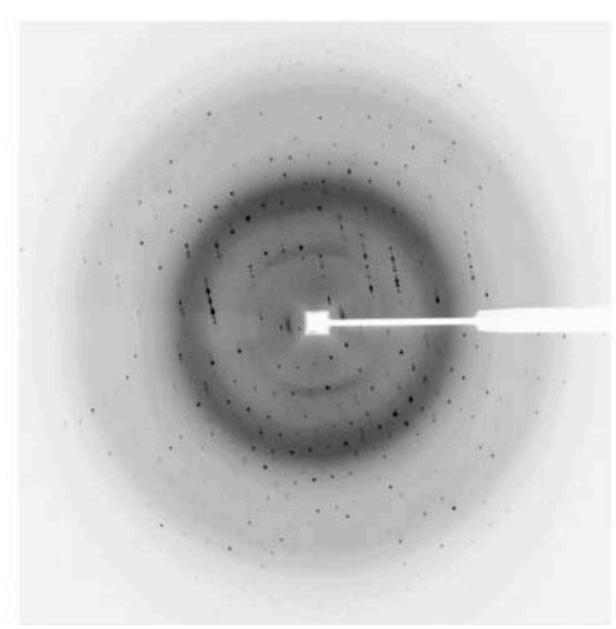

B

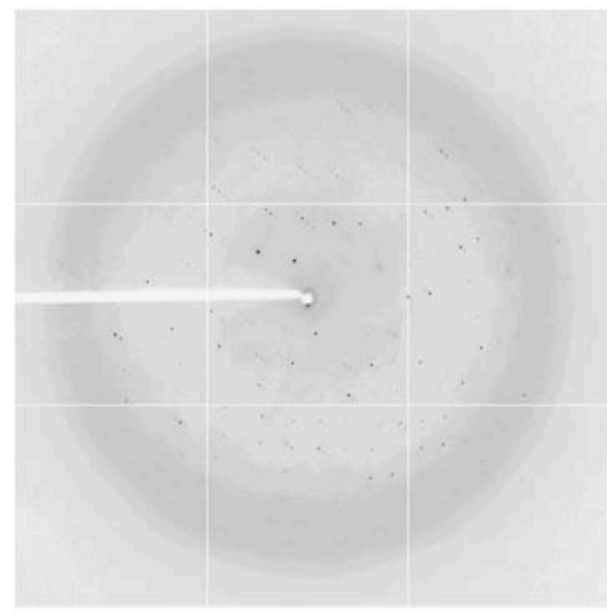

D

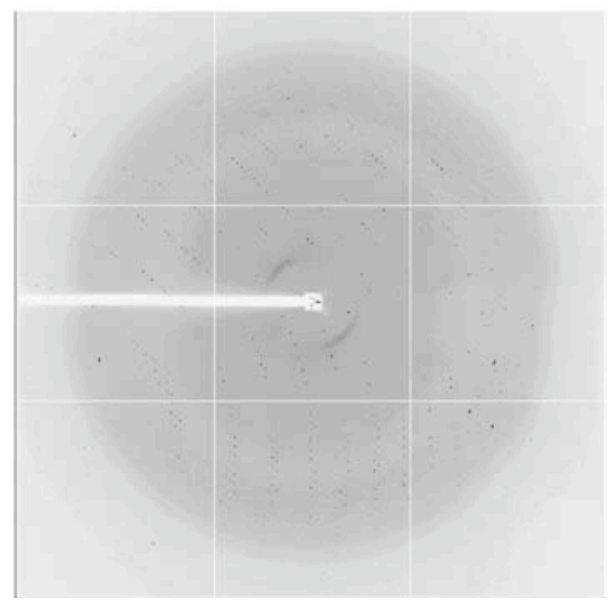

Figure A.10. Representative crystal diffraction images. The $\mathrm{SPOP}^{\mathrm{BTB}+}$ crystal diffracted to $2.5 \AA$ (A), the $\mathrm{Gig}^{1-258 \Delta \mathrm{DDGS}}$ crystal diffracted to $3.0 \AA$ (B), the $\mathrm{SPOP}^{\mathrm{MATH}}$ $\mathrm{Puc}^{\mathrm{SBC} 1}$ crystal diffracted to $1.7 \AA(\mathrm{C})$ and the $\mathrm{SPOP}^{\mathrm{MATH}-\mathrm{BTB}+}$ crystal diffracted to $2.6 \AA$ (D). 


\section{APPENDIX B. STUDIES OF INTERACTION BETWEEN E1 AND E2 OF THE NEDD8 PATHWAY}

\section{INTRODUCTION}

NEDD8 is an ubiquitin-like protein (Ubl), which shares $80 \%$ sequence similarity with ubiquitin (Ub). NEDD8 and ubiquitin are attached to the substrate by parallel, but distinct cascades of enzymes in classes known as E1, E2, and E3. The enzymatic mechanisms of $\mathrm{Ub} / \mathrm{Ubl}$ transfer from E1 to E2 are well characterized [3, 27, 28, 189]. First, E1 (Ub/Ubl activating enzyme) catalyzes the adenylation of the $\mathrm{Ub} / \mathrm{Ubl} \mathrm{C}$ terminus in an ATP-dependent manner and binds non-covalently with the Ubl-adenylate. Then the E1 catalytic cysteine attacks the Ubl-adenylate, forming a covalently linked E1-Ub/Ubl thioester. Finally, the E1 associates with E2 (Ub/Ubl conjugating enzyme) and transfer its thioester-bound Ub/Ubl to the E2's catalytic cysteine [190].

The E1 for NEDD8 pathway is heterdimer composed of two subunits, APPBP1 and UBA3. The major E2 is Ubc12. Recently, another E2 (UBE2F) was characterized with a different substrate (cullin) specificity from Ubc12 [191]. Previous studies in the lab have identified that the $\mathrm{C}$ terminus of UBA3 can interact with Ubc12 and it is important for Ubc12 NEDD8 thioester-bound complex formation [192, 193]. However, the surface of Ubc12 that interacts with UBA3 hasn't been mapped out. The main purpose of this study is to use mutational analysis to identify residues on Ubc12 that are important for E1-E2 interaction. The studies of E1-E2 interaction in NEDD8 pathway may provide a general mechanism for other Ubl pathways. It will also provide an insight into the specificity between different sets of Ubl, E1 and E2s.

\section{RESULTS}

To understand the molecular basis for E1-E2 interaction of NEDD8 pathway, we used alanine-scanning mutagenesis to examine the role of each Ubc12 surface residue in an in vitro NEDD8 transfer assay. Four regions on Ubc12 surface have been identified that are involved in Ubc12 NEDD8 thioester formation (Fig B.1). The role of each residue is understood with the crystal structures determined by Dr. Danny Huang [24, 25, 194]:

(1) Residues L32, Q35, K36, I38, E40, L41, L43, F51, D55 and L57 are involved in the interaction between Ubc12 and UBA3 $\mathrm{C}$ terminus.

(2) Q84 and H88 ineract with the adenylation domain portion of UBA3, immediately adjacent to the ATP binding site.

(3) Y130 and Y134 interact with NEDD8, which is thioester-linked on APPBP1/UBA3

(4) Asn113 and Glu117 are close to the catalytic Cystin on Ubc12, thus also have effect on Ubc12 NEDD8 formation. 


\section{METHODS}

\section{Alanine-Scanning Mutagenesis}

59 Ubc12 surface residues were selected for Alanine substitution based on crystal structure. Those residues are systematically mutated to Alanine by PCR mutagenesis in the context of Ubc12 $2^{27-\mathrm{C}}$. The mutants were cloned into expression vector pGEX4T-3 and expressed in BL21 cells. Cell lysate from 1L culture was incubated with $0.5 \mathrm{ml}$ glutathione sepharose $4 \mathrm{~B}$ at $4{ }^{\circ} \mathrm{C}$ for 1 hour. Then the beads were washed extensively with a Tris- $\mathrm{NaCl}$ buffer. Proteins were cleaved off beads by $1 \%$ thrombin at 4 degree over night.

\section{In Vitro NEDD8 Transfer Assay}

The NEDD8 transfer assays were carried out in $10 \mu \mathrm{l}$ in $50 \mathrm{mM}$ Tris-HCl, pH 7.6, $50 \mathrm{mM} \mathrm{NaCl}, 10 \mathrm{mM} \mathrm{MgCl} 2,5 \mathrm{mM}$ ATP, $1 \mathrm{mM}$ DTT, $0.3 \mathrm{U} / \mathrm{ml}$ inorganic pyrophosphatase, $0.3 \mathrm{U} / \mathrm{ml}$ creatine phosphatase, $5 \mathrm{mM}$ creatine phosphate, $2 \mathrm{mg} / \mathrm{ml}$ ovalbumin, with $1 \mathrm{nM} \mathrm{E1}$ (APPBP1/UbA3) and $5 \mathrm{mM}$ NEDD8 phosphorylated at the $\mathrm{N}$-terminal PKA site (from pGEX2TK) with [ $\gamma$-32P]ATP, at room temperature. Reactions were quenched with an equal volume of two times SDS sample buffer. Proteins were resolved by SDS-PAGE, dried and visualized by autoradiography. 


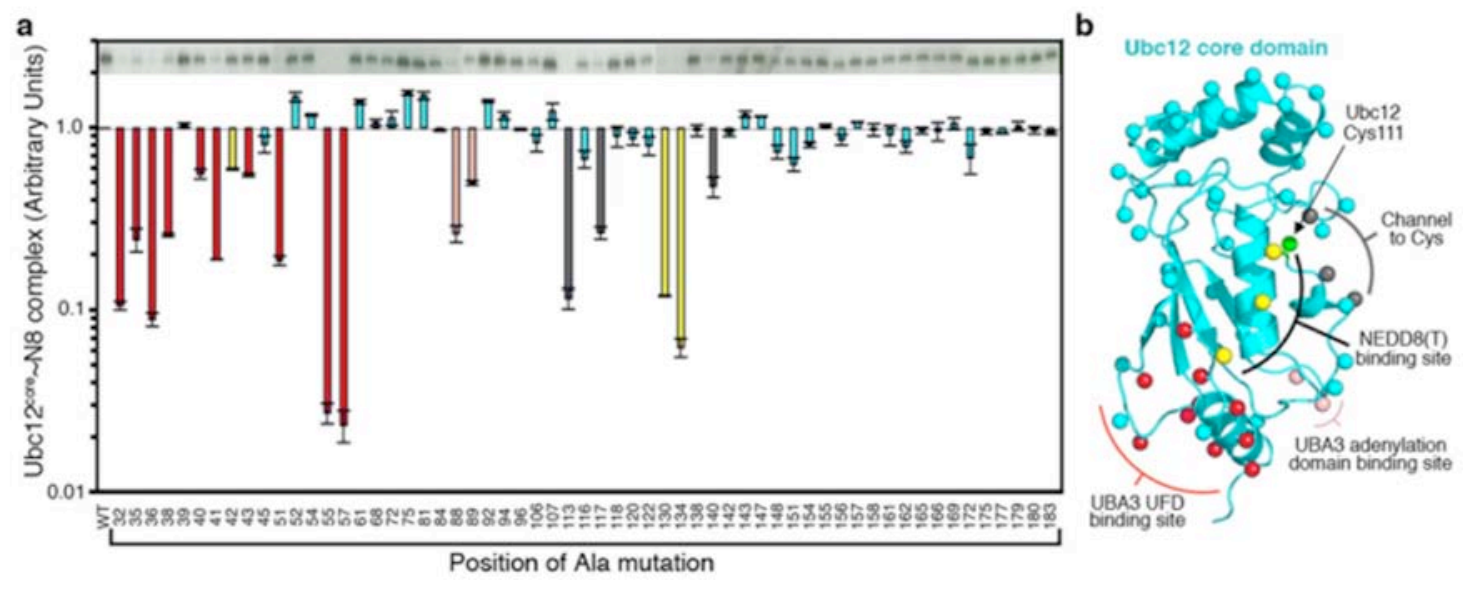

Figure B.1. Alanine-scanning mutagenesis of Ubc12's core domain: effects of alanine substitutions on APPBP1-UBA3-catalyzed formation of the Ubc12 core NEDD8 thioester product complex. (a) Effects of individual alanine substitution in Ubc12's core domain on steady-state formation of the thioester complex with NEDD8. Bars represent the average from three experiments, colored according to location on the APPBP1-UBA3 NEDD8(T)-NEDD8(A)-MgATP-Ubc12(C111A) structure[24]: red - contacts to UBA3's C terminus; yellow - contacts to NEDD8(T); pink - contacts to UBA3's adenylation domain; grey - the channel leading to Ubc12's residue 111 (the catalytic cysteine); cyan - other Ubc12 surface residues. (b) Sites of individual alanine mutations are shown as spheres on the structure of Ubc12's core domain, and are colored as in panel a. 


\section{VITA}

Min Zhuang was born in Nantong, Jiangsu Province, China in 1981. She graduated from Nantong First High School in Nantong, Jiangsu Province in June 1999. The following August, she enrolled in the Department for Intensive Instruction at Nanjing University. After achieving her Bachelor of Science degree in 2003, she joined the Interdisciplinary Program at The University of Tennessee Health Science Center for graduate studies. She is expected to obtain her Ph.D. degree in May 2009. 